\title{
Análise real e complexa generalizada de Colombeau - uma introdução
}

Danielle Gomes da Veiga

\author{
DISSERTAÇÃO APRESENTADA \\ $\mathrm{AO}$ \\ INSTITUTO DE MATEMÁTICA E ESTATÍSTICA \\ DA \\ UNIVERSIDADE DE SÃO PAULO \\ PARA \\ OBTENÇÃO DO TÍTULO DE MESTRE \\ EM \\ CIÊNCIAS
}

Área de concentração: Matemática

Orientadora: Profa. Dra. Roseli Fernandez

Durante parte da elaboração deste trabalho a autora recebeu auxílio financeiro do CNPq.

São Paulo, março de 2007. 


\section{Análise real e complexa generalizada de Colombeau - uma introdução}

Este exemplar corresponde à redação final da dissertação devidamente corrigida e defendida. por Danielle Gomes da Veiga e aprovada pela Comissão Julgadora.

São Paulo, 23 de março de 2007.

Banca Examinadora:

Profa. Dra. Roseli Fernandez (orientadora) - IME-USP

Prof. Dr. Alfredo Jorge Aragona Vallejo - IME-USP

Prof. Dr. Francisco Villarreal Alvarado - FEIS-UNESP 
A meus pais, Marly e José, a meus irmãos e à amiga Luciane. 


\section{Resumo}

Neste trabalho estudamos o conceito de diferenciabilidade para funções de uma variável generalizada ou de várias variáveis reais generalizadas; definimos, para funções de uma variável complexa generalizada, os conceitos de função holomorfa e de função analítica; e apresentamos algumas propriedades da álgebra diferencial de Colombeau (que é a imagem, por uma imersão contínua, da álgebra das funções generalizadas de Colombeau (versão simplificada)). Esses conceitos foram introduzidos e desenvolvidos por J. Aragona, R. Fernandez e S. O. Juriaans.

\section{Abstract}

In this work we study the notion of differentiability for functions of one generalized variable or of several generalized real variables; we define, for functions of one generalized complex variable, the notions of holomorphic function and of analytic function, and we present some properties of the Colombeau differential algebra (which is the image, by a continuous embedding, of algebra of Colombeau generalized funєtions). Those concepts have been introduced and developed by J. Aragona, R. Fernandez and S. O. Juriaans. 


\section{Agradecimentos}

Gostaria de agradecer, inicialmente, à Profa. Roseli Fernandez, com quem muito aprendi durante toda a elaboração desta Dissertação, pela dedicada orientação e pela sugestão do tema deste trabalho, e aos membros da Banca Examinadora pelas sugestões e pelos comentários finais feitos a esta Dissertação.

Agradeço, também, a todos os professores que contribuíram, de alguma forma, na minha formação. Em especial, agradeço, por todos os ensinamentos e incentivo, aos professores Alegria Gladys Chalom de Oliveira, Alfredo Jorge Aragona Vallejo, Antonio de Padua Franco Filho, Claudia Cueva Candido, Cristina Cerri, Daniel Victor Tausk, Deborah Martins Raphael, Eduardo do Nascimento Marcos, Francisco Rui Tavares de Almeida, Henrique Salvador Salazar Panzarelli, Lucia Satie Ikemoto Murakami, Maria Cristina Bonomi Barufi, Maria Izabel Ramalho Martins, Paulo Domingos Cordaro e Rosa Maria dos Santos Barreiro Chaves.

Desejo agradecer, ainda, aos amigos e colegas que fiz no IME-USP, de modo especial agradeço a Ana Carolina Boero, Antonio Ronaldo Gomes Garcia, Archibald de Araújo Silva, Marcus Rui Amorim, Maria do Carmo e Sávio Mendes França, pelas agradáveis conversas e por todo apoio e incentivo.

Por fim, agradeço a meus pais, a meus irmãos e a minha amiga Luciane por toda força, carinho e apoio desde sempre.

São Paulo, março de 2007.

Danielle Gomes da Veiga 


\section{Sumário}

Introdução xiii

1 Números e funções generalizadas de Colombeau $\quad 1$

1.1 Definição e algumas propriedades de $\overline{\mathbb{K}} \ldots \ldots$. . . . . . . . . . . . . 2

1.2 Uma relação de ordem parcial sobre $\overline{\mathbb{R}} \ldots \ldots \ldots$. . . . . . . . . . 8

1.3 Uma ultramétrica para $\overline{\mathbb{K}}^{n} \ldots \ldots \ldots \ldots \ldots$

1.4 Algumas propriedades de $\overline{\mathbb{K}}^{n} \ldots \ldots \ldots \ldots \ldots$

1.5 Definição e algumas propriedades de $\mathcal{G}(\Omega ; \mathbb{K}) \ldots \ldots \ldots$

$1.6 \mathrm{O}$ conjunto $\tilde{\Omega}_{c}$ e o valor pontual generalizado . . . . . . . . . . . 45

1.7 Uma ultramétrica para $\mathcal{G}(\Omega ; \mathbb{K}) \ldots \ldots \ldots \ldots \ldots$

1.8 Topologias metrizáveis sobre $\overline{\mathbb{K}}$ e sobre $\mathcal{G}(\Omega ; \mathbb{K}) \ldots \ldots \ldots$. . . . . . . . 59

$\begin{array}{lll}2 & \text { Limite e diferenciabilidade } & 61\end{array}$

2.1 Limite . . . . . . . . . . . . . . . . . . . . . . . 61

2.2 Derivada . . . . . . . . . . . . . . . . . . 65

2.3 Diferenciabilidade: funções de várias variáveis reais generalizadas . . . . . . 77

2.4 Diferenciabilidade: aplicações de variáveis reais generalizadas . . . . . . . . 82

3 Funções holomorfas e funções analíticas $\quad 87$

3.1 Dois conjuntos isometricamente isomorfos a $\overline{\mathbb{C}} \ldots$. . . . . . . . 88

3.2 Funções holomorfas e analíticas . . . . . . . . . . . . . . . . 97 
4.1 A função $\kappa_{\Omega, R}$ e a álgebra $\kappa_{\Omega, R}(\mathcal{G}(\Omega ; \mathbb{R})) \ldots \ldots \ldots \ldots$

4.2 A função $\kappa_{\Omega, \mathbb{C}}$ e a álgebra $\kappa_{\Omega, \mathbb{C}}(\mathcal{G}(\Omega ; \mathbb{C})) \ldots \ldots \ldots \ldots$

$\begin{array}{lr}\text { Índice de Símbolos e Notações } & 139\end{array}$

Referências Bibliográficas 


\section{Introdução}

A Teoria das Funções Generalizadas foi introduzida por J. F. Colombeau, aproximadamente em 1980, com a finalidade de resolver o Problema da Multiplicação de Distribuições. Desde então, a teoria vem se desenvolvendo em ritmo crescente e foram obtidas muitas aplicações em diversas áreas tais como nas equações diferenciais, na geometria e na análise funcional.

Na Teoria das Funções Generalizadas são utilizadas várias álgebras como, por exemplo, as álgebras plenas dos números e das funções generalizadas de Colombeau; as álgebras dos números e das funções generalizadas de Colombeau (versão simplificada); a álgebra plena das funções generalizadas temperadas de Colombeau e a álgebra das funções generalizadas temperadas de Colombeau (versão simplificada). As definições e algumas propriedades de cada uma dessas álgebras podem ser encontradas em [AB], [Bia] e [GKOS]. Neste trabalho serão utilizadas as álgebras dos números e das funções generalizadas de Colombeau - aqui denotadas por $\overline{\mathbb{K}}$ e $\mathcal{G}(\Omega ; \mathbb{K})$, respectivamente, onde $\mathbb{K}$ representa o corpo dos números reais ou o corpo dos números complexos e $\Omega$ denota um subconjunto aberto de $\mathbb{R}^{n}$.

Alguns resultados, no contexto clássico, dependem se há ou não uma topologia no espaço no qual se está trabalhando. Dessa forma, procurou-se munir as álgebras dos números e das funções generalizadas de Colombeau e as álgebras plenas dos números e das funções generalizadas de Colombeau com uma topologia. Primeiros resultados nessa direção, dos quais temos conhecimento, foram obtidos por H. A. Biagioni em 1990 ([Bia]). Em 1993, 
D. Scarpalezos ([Sca-1] e [Sca-2]) definiu, para as álgebras dos números e das funções generalizadas, uma ultramétrica sobre $\overline{\mathbb{K}}$ e uma sobre $\mathcal{G}(\Omega ; \mathbb{K})$ de modo que esses conjuntos, com a topologia induzida por essa ultramétrica, fossem anéis topológicos completos. Um resultado semelhante para as álgebras plenas dos números e das funções generalizadas de Colombeau foi introduzido por J. Aragona, R. Fernandez e S. O. Juriaans em [AFJ-2] e [AFJ-3]. Recentemente, J. Aragona introduziu uma topologia para a álgebra plena das funções generalizadas definidas em $\bar{O}$, denotada por $\mathcal{G}(\bar{O})$ (onde $O$ é um subconjunto aberto limitado de $\mathbb{R}^{n}$ ), e provou que, com essa topologia, $\mathcal{G}(\bar{O})$ é um espaço topológico completo. A partir dessa topologia, A. R. G. Garcia generaliza um resultado de existência e unicidade, devido a J. F. Colombeau e M. Langlais ([CL]), para um problema de valor inicial e de fronteira do tipo parabólico. Esses resultados estão em [Gar-G].

Outro importante passo dado na Teoria das Funções Generalizadas foi a introdução da definição de valor pontual generalizado - através do conjunto dos pontos compactamente suportados, denotado por $\widetilde{\Omega}_{c}$ - ambos devidos a M. Kunzinger e M. Oberguggenberger. A grande relevância desses conceitos está no fato de uma função generalizada ficar determinada por seu valor pontual generalizado ([KO]).

J. Aragona e S. O. Juriaans ([AJ]), a partir da topologia definida por D. Scarpalezos, obtiveram propriedades da estrutura algébrica e topológica da álgebra dos números generalizados e estabeleceram importantes conexões entre essas estruturas. Mais tarde, J. Aragona, R. Fernandez e S. O. Juriaans ([AFJ-1]), a partir desses resultados, introduzem uma noção de diferenciabilidade para funções definidas em subconjuntos abertos de $\overline{\mathbb{R}}^{n}$ (ou $\overline{\mathbb{C}}$ ) e a valores em $\overline{\mathbb{R}}^{m}$ (ou $\overline{\mathbb{C}}$ ), onde $\overline{\mathbb{R}}^{p}$ é munido com a topologia produto. Motivados pelo trabalho $[\mathrm{KO}]$ e tendo provado que o conjunto $\widetilde{\Omega}_{c}$ é um subconjunto aberto de $\overline{\mathbb{K}}^{n}$, lhes foi possível transformar a álgebra das funções generalizadas de Colombeau em uma álgebra de funções no sentido clássico. Mais precisamente, eles definem uma imersão contínua de $\mathcal{G}(\Omega ; \mathbb{R})$ em $\overline{\mathbb{R}}^{\widetilde{\Omega}_{c}}$ (onde $\Omega$ é um subconjunto aberto de $\mathbb{R}^{n}$ ) e uma imersão contínua de $\mathcal{G}\left(\Omega ; \mathbb{C}\right.$ ) em $\overline{\mathbb{C}}^{\widetilde{\Omega}_{c}}$ (onde $\Omega$ é um subconjunto aberto de $\mathbb{C}$ ). A imagem de cada 
uma dessas imersões é chamada de álgebra diferencial de Colombeau. Para essa álgebra os autores provam que muitos resultados válidos no contexto clássico - mas que podem ser falsos, de modo geral, para as funções definidas em subconjuntos abertos de $\overline{\mathbb{R}}^{n}$ (ou $\overline{\mathbb{C}}$ ) e a valores em $\overline{\mathbb{R}}^{m}$ (ou $\overline{\mathbb{C}}$ ) - são, com provas similares às do caso clássico, verdadeiros neste contexto. Isto nos leva a acreditar que os problemas, que são resolvidos utilizando a álgebra das funções generalizadas de Colombeau, possam ser resolvidos de um modo mais simples se utilizarmos a álgebra diferencial de Colombeau.

O objetivo desta Dissertação é introduzir esse conceito de diferenciabilidade e ser uma introdução ao estudo da álgebra diferencial de Colombeau. Os resultados que apresentamos foram extraídos dos artigos [AJ], [AFJ-1], [AFJ-2], [AFJ-3] e [AJOS], ou foram por nós obtidos a partir do estudo desses. Quanto à estrutura do trabalho, este está dividido em quatro capítulos. A seguir descreveremos cada um deles.

No capítulo 1, para facilitar a leitura do trabalho, apresentamos as álgebras dos números e das funções generalizadas de Colombeau (seções 1.1 e 1.5, respectivamente); as topologias sobre $\bar{K}$ e sobre $\mathcal{G}(\Omega ; \mathbb{K})$ devido a D. Scarpalezos mas com a abordagem feita por J. Aragona, R. Fernandez e S. O. Juriaans (seções 1.3 e 1.7, respectivamente). Essa abordagem é interessante pois não utiliza os representantes dos elementos da álgebra. Para obter propriedades da álgebra diferencial é necessário estudar as propriedades do conjunto $\widetilde{\Omega}_{c}$. Essas propriedades estão na seção 1.6. Na seção 1.4 encontram-se propriedades interessantes de $\overline{\mathbb{K}}^{n}$ como, por exemplo, as Proposições 1.4.6 e 1.4.8 relativas à convergência de série de potências envolvendo elementos de $\overline{\mathbb{K}}$. A seçẽāo 1.8, apesar de seu conteúdo não ser por nós aqui utilizado, é interessante e contém topologias metrizáveis sobre $\overline{\mathbb{K}}$ e sobre $\mathcal{G}(\Omega ; \mathbb{K})$, desenvolvidas a partir da relação de ordem parcial sobre $\overline{\mathbb{R}}$ (apresentada na seção 1.2), que coincidem com as topologias desenvolvidas por D. Scarpalezos ([AFJ-2] e

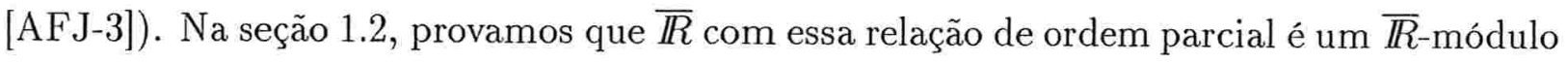
reticulado.

Iniciamos o capítulo 2 com um breve estudo de limite, pois precisamos de resultados 
que não são verdadeiros em espaços métricos em geral; como, por exemplo, que soma e produto de funções contínuas são contínuas e que o inverso de uma função contínua (cuja imagem está contida no conjunto dos invertíveis de $\overline{\mathbb{K}}$ ) é uma função contínua. Na seção 2.2 definimos o conceito de derivada para funções de uma variável generalizada. Destacamos que, neste caso, as regras de derivação são as mesmas do contexto clássico (Proposição 2.2.9, Proposição 2.2.11 e Proposição 2.2.12). Contudo, o Teorema do Valor Médio é falso neste contexto, visto que existe uma função definida em $\overline{\mathbb{R}}$, não constante, cuja derivada se anula em todo ponto de $\overline{\mathbb{R}}$ (Exemplo 2.2.4 (2)). Na seção 2.3, estudamos o conceito de diferenciabilidade para funções de várias variáveis reais generalizadas. Como no contexto clássico, essas funções são contínuas, admitem derivadas parciais e existe uma relação entre diferenciabilidade e derivadas parciais (Proposição 2.3.6 e Proposição 2.3.5). Finalizamos o capítulo introduzindo o conceito de diferenciabilidade para aplicações de variáveis reais generalizadas.

O capítulo 3 é dedicado ao estudo das funções de uma variável complexa generalizada (continuação da seção 2.2 , no caso em que $\mathbb{I}=\mathbb{C}$ ). São introduzidos os conceitos de função holomorfa (função que é derivável) e de função analítica (função que pode ser escrita em série de potências). A fim de obtermos resultados análogos aos do contexto clássico, foi necessário identificarmos $\overline{\mathbb{C}} \operatorname{com} \overline{\mathbb{R}}^{2}$ e com $\overline{\mathbb{R}}+\mathrm{i} \overline{\mathbb{R}}$. Essas identificações encontram-se na seção 3.1. A partir dessas identificações, nos foi possível estabelecer uma relação entre as condições de Cauchy-Riemann generalizadas e função holomorfa (Proposição 3.2.3 e Proposição 3.2.4). Além disso, como ocorre no contexto clássico, temos que uma função $f$ é holomorfa se, e somente se $\frac{\partial f}{\partial \bar{z}}=0$ (Proposição 3.2.7) e temos que toda função analítica é holomorfa (Teorema 3.2.11). Até o momento, não há registro, da equivalência dos conceitos de função analítica e de função holomorfa neste contexto (equivalência esta existente no contexto clássico).

No capítulo 4, apresentamos a álgebra diferencial de Colombeau e algumas de suas propriedades. Essa álgebra é a imagem de $\mathcal{G}(\Omega ; \mathbb{R})$, onde $\Omega$ é um subconjunto aberto de 
$\mathbb{R}^{n}$ (ou $\mathcal{G}(\Omega ; \mathbb{C})$, onde $\Omega$ é um subconjunto aberto de $\mathbb{C}$ ), por uma imersão contínua, em $\overline{\mathbb{R}} \tilde{\Omega}_{c}$ (ou $\overline{\mathbb{C}}^{\tilde{\Omega}_{c}}$ ). Dessa forma, as funções generalizadas de Colombeau se transformam em funções no sentido clássico. Nessa álgebra, além dos resultados anteriores, tem-se um Teorema do Valor Médio Generalizado (Teorema 4.1.6), uma Fórmula de Taylor Generalizada (Proposição 4.1.11 ), um Teorema da Aplicação Aberta Generalizado (Teorema 4.1.13), a equivalência dos conceitos de função holomorfa e de função analítica (Corolário 4.2.11) e uma caracterização para as funções holomorfas (Corolário 4.2.12).

No final da Dissertação há um índice de símbolos e notacões e as referências bibliográficas. Nessa última, além dos artigos que serviram de apoio e motivação para o nosso trabalho, listamos outros que mostram o desenvolvimento da Teoria das Funções Generalizadas. Sempre que considerarmos um subconjunto aberto, ficará implícito que ele é não vazio.

Recomendamos, para um primeiro estudo das álgebras dos números e das funções generalizadas de Colombeau (versão simplificada), a leitura de [Sil-A] e, para uma visão mais geral da Teoria das Funções Generalizadas e suas aplicações, a leitura de [GKOS].

São Paulo, março de 2007.

Danielle Gomes da Veiga 


\section{Capítulo 1}

\section{Números e funções generalizadas de}

\section{Colombeau}

Neste capítulo, apresentamos as álgebras dos números e das funções generalizadas de Colombeau (versão simplificada) e alguns resultados relativos a essas álgebras que serão úteis neste trabalho. Dentre os resultados aqui encontrados, destacamos as ultramétricas de $\overline{\mathbb{K}}$ e $\mathcal{G}(\Omega ; \mathbb{K})$ (seções 1.3 e 1.7 , respectivamente) e o conjunto $\widetilde{\Omega}_{c}$ (seção 1.6 ), pois a partir deles J. Aragona, R. Fernandez e S. O. Juriaans introduziram um conceito de diferenciabilidade. Esse conceito e algumas de suas propriedades estão no capítulo 2 . Na seção 1.2, apresentamos uma relação de ordem parcial sobre $\overline{\mathbb{R}}$ e provamos que $\overline{\mathbb{R}}$ é um $\overline{\mathbb{R}}$-módulo reticulado. Finalizamos o capítulo com a seção 1.8 na qual encontra-se, a partir da relação de ordem parcial sobre $\overline{\mathbb{R}}$, uma topologia metrizável sobre $\overline{\mathbb{K}}$ e uma sobre $\mathcal{G}(\Omega ; \mathbb{K})$. Essas topologias coincidem com as topologias induzidas pelas ultramétricas abordadas nas seções 1.3 e 1.7 , respectivamente. 


\subsection{Definição e algumas propriedades de $\overline{\mathbb{K}}$}

Nesta seção, apresentaremos a álgebra dos números generalizados de Colombeau (versão simplificada). Essa álgebra será denotada por $\overline{\mathbb{K}}$ e seus elementos serão chamados de números generalizados. Entre os resultados apresentados, destacamos o fato de que $\overline{\mathbb{K}}$ não é um corpo, as propriedades da relação de associação em $\overline{I K}$ e a definição do número generalizado $\alpha_{r}$. O fato de $\alpha_{r}$ ser um elemento invertível é fundamental para o conceito de derivada que será introduzido no capítulo 2. Durante a elaboração do trabalho, surgiram algumas propriedades da associação em $\overline{\mathbb{K}}$ envolvendo o número generalizado $\alpha_{r}$. Essas propriedades estão na Proposição 1.1.12. Os demais resultados e definições foram extraídos de [AJ] e [AJOS]. Em todo o trabalho, $\mathbb{K}$ denotará o corpo dos números reais ou o corpo dos números complexos. Além disso, utilizaremos as seguintes notações:

- $\mathbf{I}:=] 0,1]$

- $\left.\mathbf{I}_{\eta}:=\right] 0, \eta[$, para todo $\eta \in \mathbf{I}$;

- $\varepsilon \downarrow 0:=\varepsilon \rightarrow 0^{+}, \operatorname{com} \varepsilon \in \mathbf{I}$.

A seguir, recordamos a o-notação de Bachmann-Landau.

Seja $X$ um espaço topológico e $f, g: X \longrightarrow \mathbb{K}$. Diz-se que $f(x)=o(g(x))$, quando $x \longrightarrow x_{0}$ se, e somente se, $\lim _{x \rightarrow x_{0}} \frac{f(x)}{g(x)}=0$.

Definição 1.1.1 Seja $\left(\lambda_{\varepsilon}\right)_{\varepsilon} \in \mathbb{K}^{\mathbf{I}}$. Diz-se que $\left(\lambda_{\varepsilon}\right)_{\varepsilon}$ pertence a $\mathcal{E}_{M}(\mathbb{K})$ se, e somente se, $\exists a \in \mathbb{R}$ tal que $\left|\lambda_{\varepsilon}\right|=o\left(\varepsilon^{a}\right)$, quando $\varepsilon \downarrow 0$. 
Um elemento de $\mathcal{E}_{M}(\mathbb{K} K)$ é chamado de elemento moderado. Observamos que o conjunto $\mathcal{E}_{M}(\mathbb{I})$ munido das operações usuais de soma e produto de funções e produto de um elemento de $\mathbb{K}$ por uma função é uma $\mathbb{K}$-álgebra.

Definição 1.1.2 Denota-se por $\mathcal{N}(\mathbb{K})$ o conjunto das funções $\left(\lambda_{\varepsilon}\right)_{\varepsilon} \in \mathbb{K}^{\mathrm{I}}$ tais que

$$
\forall b \in \mathbb{R} \text { tem-se que }\left|\lambda_{\varepsilon}\right|=o\left(\varepsilon^{b}\right), \text { quando } \varepsilon \downarrow 0 \text {. }
$$

Um elemento de $\mathcal{N}(\mathbb{K})$ é chamado de elemento nulo. É fácil verificar que o conjunto $\mathcal{N}(\mathbb{K})$ é um ideal de $\mathcal{E}_{M}(\mathbb{K})$.

Definição 1.1.3 Denota-se por $\bar{K}$ a álgebra quociente de $\mathcal{E}_{M}(\mathbb{K})$ por $\mathcal{N}(\mathbb{K})$.

Um elemento de $\overline{\mathbb{K}}$ é chamado de número real generalizado se $\mathbb{K}=\mathbb{R}$, número complexo generalizado se $\mathbb{K}=\mathbb{C}$, ou simplesmente número generalizado. É fácil verificar que o anel quociente $\bar{K}$ é uma $\mathbb{K}$-álgebra.

Se $\lambda \in \bar{K}$, denotaremos por $\left(\lambda_{\varepsilon}\right)_{\varepsilon}$ um representante de $\lambda$. Se $\left(\lambda_{\varepsilon}\right)_{\varepsilon} \in \mathcal{E}_{M}(\mathbb{K})$, denotaremos por $\left[\left(\lambda_{\varepsilon}\right)_{\varepsilon}\right]$ a classe de $\left(\lambda_{\varepsilon}\right)_{\varepsilon}$ em $\bar{K}$, isto é, $\left[\left(\lambda_{\varepsilon}\right)_{\varepsilon}\right]=\left(\lambda_{\varepsilon}\right)_{\varepsilon}+\mathcal{N}(\mathbb{K})$.

As proposições a seguir, que em vários trabalhos são utilizadas para definir $\mathcal{E}_{M}(\mathbb{K})$ e $\mathcal{N}(\mathbb{K})$, respectivamente, nos mostram que a Definição 1.1.1 e a Definição 1.1.2 coincidem com as dadas por J. F. Colombeau. Elas serão utilizadas, ao longo desta Dissertação, sempre que for conveniente.

Proposição 1.1.4 Seja $\left(\lambda_{\varepsilon}\right)_{\varepsilon} \in \mathbb{K}^{\mathbf{I}}$. São equivalentes:

1. $\left(\lambda_{\varepsilon}\right)_{\varepsilon} \in \mathcal{E}_{M}(\mathbb{K})$;

2. existem $N \in \mathbb{N}, c>0$ e $\eta \in \mathbf{I}$ tais que $\left|\lambda_{\varepsilon}\right| \leq c \varepsilon^{-N}$, para todo $\varepsilon \in \mathbf{I}_{\eta}$. 
Demonstração: Seja $a \in \mathbb{R}$ tal que $\lim _{\varepsilon \downarrow 0} \frac{\left|\lambda_{\varepsilon}\right|}{\varepsilon^{a}}=0$. Logo, existe $\eta \in \mathbf{I}$ tal que, se $\varepsilon \in \mathbf{I}_{\eta}$, então $\frac{\left|\lambda_{\varepsilon}\right|}{\varepsilon^{a}} \leq 1$, ou seja, $\left|\lambda_{\varepsilon}\right| \leq \varepsilon^{a}$, para todo $\varepsilon \in \mathbf{I}_{\eta}$. Consideremos $q \in \mathbb{N}$ com $-q \leq a$. Como $\varepsilon^{a} \leq \varepsilon^{-q}$, pois $\left.\left.\varepsilon \in \mathbf{I}=\right] 0,1\right]$, temos que $\left|\lambda_{\varepsilon}\right| \leq \varepsilon^{-q}$, para todo $\varepsilon \in \mathbf{I}_{\eta}$; e, assim, 1. implica 2 ..

Sejam $N \in \mathbb{N}, c>0$ e $\eta \in \mathbf{I}$ como em 2.. Então, $\varepsilon^{N+1}\left|\lambda_{\varepsilon}\right| \leq c \varepsilon$, para todo $\varepsilon \in \mathbf{I}_{\eta}$. Seja $b=-N-1$. Assim, obtemos que $0 \leq \frac{\left|\lambda_{\varepsilon}\right|}{\varepsilon^{b}} \leq c \varepsilon$, para todo $\varepsilon \in \mathbf{I}_{\eta}$, e, como $\lim _{\varepsilon \downarrow 0} c \varepsilon=0$, segue que $\left|\lambda_{\varepsilon}\right|=o\left(\varepsilon^{b}\right)$, quando $\varepsilon \downarrow 0$; o que prova 1 ..

Proposição 1.1.5 $\operatorname{Seja}\left(\lambda_{\varepsilon}\right)_{\varepsilon} \in \mathbb{K}^{\mathbf{I}}$. São equivalentes:

1. $\left(\lambda_{\varepsilon}\right)_{\varepsilon} \in \mathcal{N}(\mathbb{K})$;

2. para todo $q \in \mathbb{N}$ existem $c>0$ e $\eta \in \mathbf{I}$ tais que $\left|\lambda_{\varepsilon}\right| \leq c \varepsilon^{q}$, para todo $\varepsilon \in \mathbf{I}_{\eta}$.

Demonstração: Suponhamos que $\left(\lambda_{\varepsilon}\right)_{\varepsilon} \in \mathcal{N}(\mathbb{K})$. Logo, dado $q \in \mathbb{N}$ temos que $\lim _{\varepsilon \downarrow 0} \frac{\left|\lambda_{\varepsilon}\right|}{\varepsilon^{q}}=0$. Portanto, existe $\eta \in \mathbf{I}$ tal que, se $\varepsilon \in \mathbf{I}_{\eta}$, então $\left|\lambda_{\varepsilon}\right| \leq \varepsilon^{q}$; o que prova 2 ..

Suponhamos que a afirmação 2. seja verdadeira. Seja $b \in \mathbb{R}$ e consideremos $p \in \mathbb{N}$ com $p>b$. Logo, existem $c>0$ e $\eta \in \mathbf{I}$ tais que $\left|\lambda_{\varepsilon}\right| \leq c \varepsilon^{p}$, para todo $\varepsilon \in \mathbf{I}_{\eta}$. Portanto, $0 \leq\left|\lambda_{\varepsilon}\right| \varepsilon^{-b} \leq c \varepsilon^{p-b}$, para todo $\varepsilon \in \mathbf{I}_{\eta}$, e, como $\lim _{\varepsilon \downarrow 0} c \varepsilon^{p-b}=0$, obtemos que $\left|\lambda_{\varepsilon}\right|=o\left(\varepsilon^{b}\right)$, quando $\varepsilon \downarrow 0$; e, assim, 2. implica 1 ..

É fácil verificar que a aplicação $h: \mathbb{K} \longrightarrow \overline{\mathbb{K}}$ dada por $h(k):=\left[\left(k_{\varepsilon}\right)_{\varepsilon}\right]$, onde $k_{\varepsilon}=k$, para todo $\varepsilon \in \mathbf{I}$, é um homomorfismo injetor. Assim, podemos identificar $\mathbb{K} \operatorname{com} h(\mathbb{K})$ e escrevermos $\mathbb{K} \subset \bar{K}$.

\section{Observação 1.1.6 $\overline{\mathbb{K}}$ não é um corpo.}

De fato, seja, para cada $\varepsilon \in \mathbf{I}$,

$$
x_{\varepsilon}:=\left\{\begin{array}{ll}
0 & , \text { se } \varepsilon=\frac{1}{n}, n \in \mathbb{N}^{*} \\
1 & , \text { caso contrário }
\end{array} .\right.
$$


É imediato verificar que $\left(x_{\varepsilon}\right)_{\varepsilon} \in \mathcal{E}_{M}(\mathbb{K} K)$ e, portanto, podemos considerar $x:=\left[\left(x_{\varepsilon}\right)_{\varepsilon}\right] \in \overline{\mathbb{I} K}$. Como, claramente, $\left(x_{\varepsilon}\right)_{\varepsilon} \notin \mathcal{N}(\mathbb{K})$, segue que $x \neq 0$. Suponhamos que exista $y:=\left[\left(y_{\varepsilon}\right)_{\varepsilon}\right] \in$ $\overline{\mathbb{K}}, \operatorname{com} x y=1$. Então existe $\left(m_{\varepsilon}\right)_{\varepsilon} \in \mathcal{N}(\mathbb{K})$ tal que $x_{\varepsilon} y_{\varepsilon}+m_{\varepsilon}=1$, para todo $\varepsilon \in \mathbf{I}$. Logo, $m_{\varepsilon}=1$ para $\varepsilon=\frac{1}{n}, n \in \mathbb{N}^{*}$, o que é um absurdo pois $\left(m_{\varepsilon}\right)_{\varepsilon} \in \mathcal{N}(\mathbb{K})$.

Definição 1.1.7 Define-se $\operatorname{Inv}(\overline{\mathbb{K}}):=\left\{x \in \overline{\mathbb{K}}^{*}: \exists y \in \bar{K}\right.$ tal que $\left.x y=1\right\}$.

Um elemento de $\operatorname{Inv}(\overline{\mathbb{K}})$ é chamado de invertivel. É claro que $\mathbb{K}^{*} \subset \operatorname{Inv}(\bar{K})$.

Observamos que, se $x \in \operatorname{Inv}(\bar{K})$, então existe um único $y \in \bar{K}$ tal que $x y=1$, o qual será denotado por $x^{-1}$ ou por $\frac{1}{x}$ e será chamado de inverso multiplicativo de $x$. Além disso, para $x \in \operatorname{Inv}(\bar{K})$ e $y \in \bar{K}$, escreveremos $\frac{y}{x}$ ao invés de $y x^{-1}$, sempre que for conveniente.

Definição 1.1.8 Seja $r \in \mathbb{R}$. A classe, em $\overline{\mathbb{K}}$, do elemento moderado $\left(\varepsilon^{r}\right)_{\varepsilon}$ é denotada por $\alpha_{r}$, isto é, $\alpha_{r}:=\left[\left(\varepsilon^{r}\right)_{\varepsilon}\right]$.

Proposição 1.1.9 O elemento $\alpha_{r} \in \operatorname{Inv}(\bar{K})$, para todo $r \in \mathbb{R}$.

Demonstração: Seja $r \in \mathbb{R}$. Temos que

$$
\alpha_{r} \alpha_{-r}=\left[\left(\varepsilon^{r}\right)_{\varepsilon}\right]\left[\left(\varepsilon^{-r}\right)_{\varepsilon}\right]=\left[\left(\varepsilon^{r} \varepsilon^{-r}\right)_{\varepsilon}\right]=\left[(1)_{\varepsilon}\right]=1
$$

e, portanto, $\alpha_{r} \in \operatorname{Inv}(\overline{\mathbb{K}})$.

Apresentaremos, a seguir, a relação de associação em $\bar{K}$. Tal relação será utilizada ao longo de toda a Dissertação.

Definição 1.1.10 Sejam $x, y \in \bar{K}$. Diz-se que $x$ é associado a zero, e denota-se por $x \approx 0$, se para algum representante $\left(x_{\varepsilon}\right)_{\varepsilon}$ de $x$ tem-se que $\lim _{\varepsilon \downarrow 0} x_{\varepsilon}=0$. Diz-se que $x$ é associado a $y$, e denota-se por $x \approx y$, se $x-y \approx 0$. 
Proposição 1.1.11 Seja $x \in \bar{K}$. Então $x \approx 0$ se, e somente se, para todo representante $\left(x_{\varepsilon}\right)_{\varepsilon}$ de $x$ tem-se que $\lim _{\varepsilon \downarrow 0} x_{\varepsilon}=0$.

Demonstração: Sejam $\left(x_{1 \varepsilon}\right)_{\varepsilon}$ um representante de $x$ tal que $\lim _{\varepsilon \downarrow 0} x_{1 \varepsilon}=0$ e $\left(x_{\varepsilon}\right)_{\varepsilon}$ um representante qualquer de $x$. Como $\left(x_{\varepsilon}-x_{1 \varepsilon}\right)_{\varepsilon} \in \mathcal{N}(\mathbb{K})$, existe $\eta \in \mathbb{N}$ tal que

$$
\left|x_{\varepsilon}-x_{1 \varepsilon}\right| \leq \varepsilon, \quad \forall \varepsilon \in \mathbf{I}_{\eta}
$$

Notemos que

$$
\left|x_{\varepsilon}\right| \leq\left|x_{\varepsilon}-x_{1 \varepsilon}\right|+\left|x_{1 \varepsilon}\right| \leq \varepsilon+\left|x_{1 \varepsilon}\right|, \quad \forall \varepsilon \in \mathbf{I}_{\eta},
$$

e, como $\lim _{\varepsilon \downarrow 0}\left(\varepsilon+\left|x_{1 \varepsilon}\right|\right)=0$, segue que $\lim _{\varepsilon \downarrow 0} x_{\varepsilon}=0$.

A recíproca segue diretamente da definição de elemento associado.

Em seguida, apresentaremos algumas propriedades da relação de associação em $\overline{\mathbb{K}}$ as quais serão utililizadas, principalmente, na seção 1.3.

Proposição 1.1.12 Sejam $x, y \in \bar{K}, k \in \mathbb{K}^{*}$ e $r, s \in \mathbb{R}$. Tem-se que:

1. $x \approx 0$ se, e somente se, $k x \approx 0$;

2. se $x \approx 0$ e $y \approx 0$, então $x+y \approx 0$;

3. se $x \approx 0$ e $y \approx 0$, então $x y \approx 0$;

4. $x \alpha_{-r} \approx 0$, para todo $r \in \mathbb{R}$, se, e somente se, $x=0$;

5. se $x \alpha_{-r} \approx 0$ e $s \leq r$, então $x \alpha_{-s} \approx 0$;

6. $\left[\left(k_{\varepsilon}\right)_{\varepsilon}\right] \alpha_{-r} \approx 0$ se, e somente se, $r<0$, onde $k_{\varepsilon}=k$, para todo $\varepsilon \in \mathbf{I}$;

7. se $x \alpha_{-r} \approx 0, y \alpha_{-s} \approx 0$ e $s \leq r$, então $(x+y) \alpha_{-s} \approx 0$;

8. se $x \alpha_{-r} \approx 0$ e $y \alpha_{-s} \approx 0$, então $x y \alpha_{-(r+s)} \approx 0$. 
Demonstração: As afirmações 1., 2. e 3. são conseqüências diretas da Definição 1.1 .10 e das propriedades de limite. A seguir, provaremos as demais afirmações.

Sejam $\left(x_{\varepsilon}\right)_{\varepsilon}$ e $\left(y_{\varepsilon}\right)_{\varepsilon}$ representantes de $x$ e $y$, respectivamente.

4. Notemos que

$$
x=0 \Longleftrightarrow \lim _{\varepsilon \downarrow 0} \frac{\left|x_{\varepsilon}\right|}{\varepsilon^{r}}=0, \forall r \in \mathbb{R} \Longleftrightarrow \lim _{\varepsilon \downarrow 0} x_{\varepsilon} \varepsilon^{-r}=0, \forall r \in \mathbb{R} \Longleftrightarrow x \alpha_{-r} \approx 0, \forall r \in \mathbb{R} .
$$

5. Se $s \leq r$, então $\varepsilon^{r} \leq \varepsilon^{s}$. Assim, temos que

$$
0 \leq \frac{\left|x_{\varepsilon}\right|}{\varepsilon^{s}} \leq \frac{\left|x_{\varepsilon}\right|}{\varepsilon^{r}}, \forall \varepsilon \in \mathbf{I},
$$

e, como $\lim _{\varepsilon \downarrow 0} \frac{\left|x_{\varepsilon}\right|}{\varepsilon^{r}}=0$, segue que $x \alpha_{-s} \approx 0$.

6. É suficiente observamos que

$$
\lim _{\varepsilon \downarrow 0} k_{\varepsilon} \varepsilon^{-r}=\lim _{\varepsilon \downarrow 0} k \varepsilon^{-r}=0 \Longleftrightarrow r<0 .
$$

7. Notemos, por (5), que $x \alpha_{-s} \approx 0$. Logo, por (2), obtemos que $x \alpha_{-s}+y \alpha_{-s}=$ $(x+y) \alpha_{-s} \approx 0$.

8. Por (3), temos que $x \alpha_{-r} y \alpha_{-s}=x y \alpha_{-(r+s)} \approx 0$.

Finalizamos esta seção apresentando a definição de módulo de um número generalizado. Para tanto, observamos que, se $\left(x_{\varepsilon}\right)_{\varepsilon}$ e $\left(x_{1 \varepsilon}\right)_{\varepsilon}$ são representantes de $x \in \bar{K}$, então $\left(\left|x_{\varepsilon}\right|-\left|x_{1 \varepsilon}\right|\right)_{\varepsilon} \in \mathcal{N}(\mathbb{R})$ pois ||$x_{\varepsilon}|-| x_{1 \varepsilon}|| \leq\left|x_{\varepsilon}-x_{1 \varepsilon}\right|$, para todo $\varepsilon \in \mathbf{I}$. Logo, faz sentido a seguinte definição.

Definição 1.1.13 Para cada $x \in \bar{K}$, chama-se de módulo generalizado de $x$, e denota-se por $|x|$, o elemento de $\overline{\mathbb{R}}$ dado por $|x|:=\left[\left(\left|x_{\varepsilon}\right|\right)_{\varepsilon}\right]$, onde $\left(x_{\varepsilon}\right)_{\varepsilon}$ é um representante qualquer de $x$. 
Algumas propriedades da função $|\cdot|: x \in \bar{K} \longmapsto|x| \in \overline{\mathbb{R}}$ serão apresentadas nas Proposições 1.2 .9 e 1.2.10.

\subsection{Uma relação de ordem parcial sobre $\bar{R}$}

Nesta seção, apresentamos a relação de ordem parcial sobre $\overline{\mathbb{R}}$ encontrada em [GKOS] e desenvolvida por J. Aragona, S. O. Juriaans, O. Oliveira e D. Scarpalezos. Além disso, veremos algumas de suas propriedades (extraídas de [AJOS]) e provaremos que $\overline{\mathbb{R}}$ munido com essa relação de ordem é um $\overline{\mathbb{R}}$-módulo reticulado (resultado este que surgiu durante nossos estudos).

Lema 1.2.1 Seja $x \in \overline{\mathbb{R}}$. São equivalentes:

1. existe um representante $\left(x_{\varepsilon}\right)_{\varepsilon}$ de $x$ tal que

$$
\forall b>0, \exists \eta_{b} \in \mathbf{I} \text { de modo que } x_{\varepsilon} \geq-\varepsilon^{b}, \forall \varepsilon \in \mathbf{I}_{\eta_{b}} ;
$$

2. todo representante $\left(x_{\varepsilon}\right)_{\varepsilon}$ de $x$ satisfaz $(1.1)$;

3. existe um representante $\left(x_{\varepsilon}\right)_{\varepsilon}$ de $x$ e existe $\eta \in \mathbf{I}$ tais que $x_{\varepsilon} \geq 0$, para todo $\varepsilon \in \mathbf{I}_{\eta}$;

4. existe um representante $\left(x_{\varepsilon}\right)_{\varepsilon}$ de $x$ tal que $x_{\varepsilon} \geq 0$, para todo $\varepsilon \in \mathbf{I}$.

Demonstração: É claro que 2. implica 1. e que 4. implica 3.. Assim, basta mostrarmos que 1. implica 4. e que 3. implica 2. .

Seja $\left(x_{\varepsilon}\right)_{\varepsilon}$ um representante de $x$ que satisfaça (1.1).

Consideremos $A:=\left\{\varepsilon \in \mathbf{I}: x_{\varepsilon} \leq 0\right\}$. Logo, se $A=\emptyset$, então $x_{\varepsilon}>0$, para todo $\varepsilon \in \mathbf{I}$, e, assim, 4. é verdadeira. Suponhamos, então, que $A \neq \varnothing$ e seja $x_{1 \varepsilon}:=0$, se $\varepsilon \in A$, e $x_{1 \varepsilon}:=x_{\varepsilon}$, se $\varepsilon \in \mathbf{I} \backslash A$. Logo, pela definição de $A$, temos que $x_{1 \varepsilon} \geq 0$, para todo $\varepsilon \in \mathbf{I}$. 
Afirmamos que $\left(x_{1 \varepsilon}-x_{\varepsilon}\right)_{\varepsilon} \in \mathcal{N}(\mathbb{R})$, ou seja, que $\left(x_{1 \varepsilon}\right)_{\varepsilon}$ é um representante de $x$. De fato, seja $q \in \mathbb{N}$ e consideremos $b>q$. Como $\left(x_{\varepsilon}\right)_{\varepsilon}$ satisfaz (1.1), existe $\eta_{b} \in \mathbf{I}$ tal que $x_{\varepsilon} \geq-\varepsilon^{b}$, para todo $\varepsilon \in \mathbf{I}_{\eta_{b}}$. Assim, obtemos que $\left|x_{1 \varepsilon}-x_{\varepsilon}\right|=\left|-x_{\varepsilon}\right|=-x_{\varepsilon} \leq \varepsilon^{b} \leq \varepsilon^{q}$, se $\varepsilon \in A \cap \mathbf{I}_{\eta_{b}}$, e $\left|x_{1 \varepsilon}-x_{\varepsilon}\right|=0 \leq \varepsilon^{q}$, se $\varepsilon \in \mathbf{I}_{\eta_{b}} \backslash A$, ou seja, $\left(x_{1 \varepsilon}\right)_{\varepsilon}$ é um representante de $x$ (Proposição 1.1.5). Logo, 4. é verdadeira.

A seguir, mostraremos que 3. implica $2 .$.

Sejam $\left(x_{2 \varepsilon}\right)_{\varepsilon}$ um representante de $x$ e $\eta \in \mathbf{I}$ tais que $x_{2 \varepsilon} \geq 0$, para todo $\varepsilon \in \mathbf{I}_{\eta}$, e seja $\left(x_{3 \varepsilon}\right)_{\varepsilon}$ um representante qualquer de $x$. Consideremos $b>0$. Como $\left(x_{2 \varepsilon}-x_{3 \varepsilon}\right)_{\varepsilon} \in \mathcal{N}(\mathbb{R})$, existe $\eta_{b} \in \mathbf{I}$ tal que $\eta_{b}<\eta$ e $\left|x_{2 \varepsilon}-x_{3 \varepsilon}\right| \leq \varepsilon^{b}$, para todo $\varepsilon \in \mathbf{I}_{\eta_{b}}$. Assim, $x_{2 \varepsilon}-x_{3 \varepsilon} \leq$ $\left|x_{2 \varepsilon}-x_{3 \varepsilon}\right| \leq \varepsilon^{b}$, para todo $\varepsilon \in \mathbf{I}_{\eta_{b}}$, e, conseqüentemente, $x_{3 \varepsilon} \geq x_{2 \varepsilon}-\varepsilon^{b} \geq-\varepsilon^{b}$, para todo $\varepsilon \in \mathbf{I}_{\eta_{b}}$.

Definição 1.2.2 Sejam $x, y \in \bar{R}$. Diz-se que

(i) $x$ é não-negativo, quase-positivo ou q-positivo, e denota-se por $x \geq 0$, se $x$ tem um representante satisfazendo uma das condições do Lema 1.2.1;

(ii) $x$ é não-positivo, quase-negativo ou q-negativo se $-x$ é q-positivo;

(iii) $x \geq y$ se, e somente se, $x-y$ é q-positivo.

Às vezes, escreveremos $y \leq x$ ao invés de $x \geq y$.

Do Lema 1.2.1 e da Definição 1.2.2, obtemos:

Observação 1.2.3 Sejam $x, y, z \in \overline{\mathbb{R}}$. Tem-se que

(a) $x \geq y$ se, e somente se, para quaisquer $\left(x_{\varepsilon}\right)_{\varepsilon}$ e $\left(y_{\varepsilon}\right)_{\varepsilon}$ representantes de $x$ e $y$, respectivamente, e para todo $b>0$ existe $\eta_{b} \in \mathbf{I}$ de modo que $x_{\varepsilon}-y_{\varepsilon} \geq-\varepsilon^{b}$, para todo $\varepsilon \in \mathbf{I}_{\eta_{b}}$ 
(b) $x \geq y$ se, e somente se, uma das seguintes afirmações é verdadeira:

(b1) existem $\left(x_{\varepsilon}\right)_{\varepsilon}$ e $\left(y_{\varepsilon}\right)_{\varepsilon}$ representantes de $x$ e $y$, respectivamente, e existe $\eta \in \mathbf{I}$ tais que $x_{\varepsilon} \geq y_{\varepsilon}$, para todo $\varepsilon \in \mathbf{I}_{\eta}$;

(b2) existem $\left(x_{\varepsilon}\right)_{\varepsilon} e\left(y_{\varepsilon}\right)_{\varepsilon}$ representantes de $x$ e $y$, respectivamente, tais que $x_{\varepsilon} \geq y_{\varepsilon}$, para todo $\varepsilon \in \mathbf{I}$;

(c) se $x \geq y$, então $x+z \geq y+z$;

(d) se $z \geq 0$ e $x \geq y$, então $x z \geq y z$.

Proposição 1.2.4 O conjunto $\overline{\mathbb{R}}$ com a relação $\geq$ é um conjunto parcialmente ordenado.

Demonstração: Sejam $x, y$ e $z$ elementos quaisquer de $\overline{\mathbb{R}}$. É claro que $x \geq x$, pois para todo representante $\left(x_{\varepsilon}\right)_{\varepsilon}$ de $x$ tem-se que $x_{\varepsilon} \geq x_{\varepsilon}$, para todo $\varepsilon \in \mathbf{I}$.

Suponhamos que $x \geq y$ e $y \geq x$. Então existem $\left(m_{\varepsilon}\right)_{\varepsilon},\left(n_{\varepsilon}\right)_{\varepsilon} \in \mathcal{N}(\mathbb{R})$ e $\left(x_{\varepsilon}\right)_{\varepsilon},\left(y_{\varepsilon}\right)_{\varepsilon}$ representantes de $x$ e $y$, respectivamente, tais que

$$
x_{\varepsilon} \geq y_{\varepsilon} \quad \text { e } \quad y_{\varepsilon}+m_{\varepsilon} \geq x_{\varepsilon}+n_{\varepsilon}, \quad \forall \varepsilon \in \mathbf{I},
$$

logo,

$$
\left|x_{\varepsilon}-y_{\varepsilon}\right|=x_{\varepsilon}-y_{\varepsilon} \leq m_{\varepsilon}-n_{\varepsilon}=\left|m_{\varepsilon}-n_{\varepsilon}\right|, \quad \forall \varepsilon \in \mathbf{I}
$$

Seja $q \in \mathbb{N}$. Como $\left(m_{\varepsilon}-n_{\varepsilon}\right)_{\varepsilon} \in \mathcal{N}(\mathbb{R})$, existe $\eta \in \mathbf{I}$ tal que

$$
\left|m_{\varepsilon}-n_{\varepsilon}\right| \leq \varepsilon^{q}, \quad \forall \varepsilon \in \mathbf{I}_{\eta}
$$

Logo, $\left|x_{\varepsilon}-y_{\varepsilon}\right| \leq \varepsilon^{q}$, para todo $\varepsilon \in \mathbf{I}_{\eta}$, e, assim, $x=y$ (Proposição 1.1.5).

Finalmente, se $x \geq y$ e $y \geq z$, existem $\left(m_{\varepsilon}\right)_{\varepsilon} \in \mathcal{N}(\mathbb{R})$ e $\left(x_{\varepsilon}\right)_{\varepsilon},\left(y_{\varepsilon}\right)_{\varepsilon},\left(z_{\varepsilon}\right)_{\varepsilon}$ representantes de $x, y$ e $z$, respectivamente, tais que

$$
x_{\varepsilon} \geq y_{\varepsilon} \quad \text { e } \quad y_{\varepsilon}+m_{\varepsilon} \geq z_{\varepsilon}, \quad \forall \varepsilon \in \mathbf{I},
$$


desse modo, temos que

$$
x_{\varepsilon}+m_{\varepsilon} \geq y_{\varepsilon}+m_{\varepsilon} \geq z_{\varepsilon}, \quad \forall \varepsilon \in \mathbf{I},
$$

ou seja, $x \geq z$.

Observação 1.2.5 O conjunto $\overline{\mathbb{R}}$ com a relação $\geq$ não é um conjunto totalmente ordenado.

De fato, seja $x_{\varepsilon}:=\varepsilon \operatorname{sen}\left(\varepsilon^{-1}\right)$, para todo $\varepsilon \in \mathbf{I}$. Como $\left|x_{\varepsilon}\right| \leq 1$, para todo $\varepsilon \in \mathbf{I}$, temos que $\left(x_{\varepsilon}\right)_{\varepsilon} \in \mathcal{E}_{M}(\mathbb{K})$ e, portanto, podemos considerar $x:=\left[\left(x_{\varepsilon}\right)_{\varepsilon}\right] \in \overline{\mathbb{R}}$. Mostraremos que $x$ não é q-positivo e nem q-negativo, ou seja, que $x$ e $-x$ não são q-positivos.

Suponhamos que $x \geq 0$. Então, dado $b=2$ existe $\eta_{b} \in \mathbf{I}$ tal que $\varepsilon \operatorname{sen}\left(\varepsilon^{-1}\right) \geq-\varepsilon^{2}$, para todo $\varepsilon \in \mathbf{I}_{\eta_{b}}$. Seja $k \in \mathbb{N}$ tal que $\varepsilon_{k}:=\left(\frac{3 \pi}{2}+2 k \pi\right)^{-1}<\eta_{b}$. Então $\varepsilon_{k}=-\operatorname{sen}\left(\varepsilon_{k}^{-1}\right) \varepsilon_{k} \leq \varepsilon_{k}^{2}$ o que é um absurdo e, portanto, $x$ não é q-positivo.

Suponhamos que $-x=\left[\left(-x_{\varepsilon}\right)_{\varepsilon}\right] \geq 0$. Então, dado $c=2$ existe $\eta_{c} \in \mathbf{I}$ tal que $-\varepsilon \operatorname{sen}\left(\varepsilon^{-1}\right) \geq-\varepsilon^{2}$, para todo $\varepsilon \in \mathbf{I}_{\eta_{c}}$. Seja $j \in \mathbb{N}$ tal que $\varepsilon_{j}:=\left(\frac{\pi}{2}+2 j \pi\right)^{-1}<\eta_{c}$. Então $\varepsilon_{j}=\operatorname{sen}\left(\varepsilon_{j}^{-1}\right) \varepsilon_{j} \leq \varepsilon_{j}^{2}$ o que é um absurdo e, portanto, $-x$ não é q-positivo.

Proposição 1.2.6 Se $x, y \in \mathbb{R} e 0 \leq x \leq y$, então existem $\left(x_{\varepsilon}\right)_{\varepsilon} e\left(y_{\varepsilon}\right)_{\varepsilon}$ representantes de $x$ e $y$, respectivamente, tais que $0 \leq x_{\varepsilon} \leq y_{\varepsilon}$, para todo $\varepsilon \in \mathbf{I}$.

Demonstração: De $0 \leq x$ e $x \leq y$ existem $\left(m_{\varepsilon}\right)_{\varepsilon} \in \mathcal{N}(\mathbb{K})$ e $\left(x_{\varepsilon}\right)_{\varepsilon},\left(y_{1 \varepsilon}\right)_{\varepsilon}$ representantes de $x$ e $y$, respectivamente, tais que

$$
0 \leq x_{\varepsilon} \quad \text { e } \quad x_{\varepsilon}+m_{\varepsilon} \leq y_{1 \varepsilon}, \forall \varepsilon \in \mathbf{I},
$$

e, assim,

$$
0 \leq x_{\varepsilon} \leq y_{1 \varepsilon}-m_{\varepsilon}, \forall \varepsilon \in \mathbf{I}
$$

Desse modo, como $\left(m_{\varepsilon}\right)_{\varepsilon} \in \mathcal{N}(\mathbb{R})$, temos que $\left(y_{1 \varepsilon}-m_{\varepsilon}\right)_{\varepsilon}$ é um representante de $y$ e, portanto, basta definirmos $y_{\varepsilon}:=y_{1 \varepsilon}-m_{\varepsilon}$, para todo $\varepsilon \in \mathbf{I}$. 
Proposição 1.2.7 O conjunto $\overline{\mathbb{R}}$ munido com a relação $\geq$ é um reticulado, isto é, dados $x, y \in \overline{\mathbb{R}}$ tem-se que:

1. existe $z \in \overline{\mathbb{R}}$ tal que $x \leq z$ e $y \leq z$ e, se $j \in \overline{\mathbb{R}}$ é tal que $x \leq j$ e $y \leq j$, então $z \leq j$;

2. existe $w \in \overline{\mathbb{R}}$ tal que $w \leq x$ e $w \leq y$ e, se $k \in \overline{\mathbb{R}}$ é tal que $k \leq x$ e $k \leq y$, então $k \leq w$.

$O$ elemento $z$ é denotado por $x \vee y$ e o elemento $w$ por $x \wedge y$.

Demonstração: Sejam $\left(x_{\varepsilon}\right)_{\varepsilon}$ e $\left(y_{\varepsilon}\right)_{\varepsilon}$ representantes de $x$ e $y$, respectivamente. Consideremos $z_{\varepsilon}:=\max \left\{x_{\varepsilon}, y_{\varepsilon}\right\}$ e $w_{\varepsilon}:=\min \left\{x_{\varepsilon}, y_{\varepsilon}\right\}$, para cada $\varepsilon \in \mathbf{I}$. Como $\left|z_{\varepsilon}\right| \leq\left|x_{\varepsilon}\right|+\left|y_{\varepsilon}\right|$ e $\left|w_{\varepsilon}\right| \leq\left|x_{\varepsilon}\right|+\left|y_{\varepsilon}\right|$, temos que $\left(z_{\varepsilon}\right)_{\varepsilon}$ e $\left(w_{\varepsilon}\right)_{\varepsilon}$ são elementos moderados. Sejam $z:=\left[\left(z_{\varepsilon}\right)_{\varepsilon}\right]$ e $w:=\left[\left(w_{\varepsilon}\right)_{\varepsilon}\right]$. É claro que $w \leq x \leq z$ e $w \leq y \leq z$.

Consideremos $j, k \in \mathbb{R}$ como em 1. e 2. e sejam $\left(j_{\varepsilon}\right)_{\varepsilon}$ e $\left(k_{\varepsilon}\right)_{\varepsilon}$ representantes de $j$ e $k$, respectivamente, e $\left(l_{\varepsilon}\right)_{\varepsilon},\left(m_{\varepsilon}\right)_{\varepsilon},\left(n_{\varepsilon}\right)_{\varepsilon},\left(p_{\varepsilon}\right)_{\varepsilon},\left(q_{\varepsilon}\right)_{\varepsilon},\left(r_{\varepsilon}\right)_{\varepsilon}$ pertencentes a $\mathcal{N}(\mathbb{R})$ tais que

$$
x_{\varepsilon}+l_{\varepsilon} \leq j_{\varepsilon}, \quad y_{\varepsilon}+m_{\varepsilon} \leq j_{\varepsilon}+n_{\varepsilon}, \quad k_{\varepsilon} \leq x_{\varepsilon}+p_{\varepsilon} \quad \text { e } \quad k_{\varepsilon}+r_{\varepsilon} \leq y_{\varepsilon}+q_{\varepsilon}
$$

para todo $\varepsilon \in \mathbf{I}$. Logo, temos que

$$
x_{\varepsilon} \leq j_{\varepsilon}-l_{\varepsilon}, \quad y_{\varepsilon} \leq j_{\varepsilon}+n_{\varepsilon}-m_{\varepsilon}, \quad x_{\varepsilon} \geq k_{\varepsilon}-p_{\varepsilon} \quad \text { e } \quad y_{\varepsilon} \geq k_{\varepsilon}+r_{\varepsilon}-q_{\varepsilon}
$$

para todo $\varepsilon \in \mathbf{I}$.

Sejam, para cada $\varepsilon \in \mathbf{I}$,

$$
j_{1 \varepsilon}:=\left\{\begin{array}{ll}
j_{\varepsilon}-l_{\varepsilon} & , \text { se } x_{\varepsilon}>y_{\varepsilon} \\
j_{\varepsilon}+n_{\varepsilon}-m_{\varepsilon} & , \text { se } y_{\varepsilon} \geq x_{\varepsilon}
\end{array} \quad \text { e } \quad k_{1 \varepsilon}:=\left\{\begin{array}{ll}
k_{\varepsilon}-p_{\varepsilon} & \text {, se } x_{\varepsilon}<y_{\varepsilon} \\
k_{\varepsilon}+r_{\varepsilon}-q_{\varepsilon} & , \text { se } y_{\varepsilon} \leq x_{\varepsilon}
\end{array} .\right.\right.
$$

Como $\left|j_{1 \varepsilon}-j_{\varepsilon}\right| \leq\left|l_{\varepsilon}\right|+\left|n_{\varepsilon}\right|+\left|m_{\varepsilon}\right|$ e $\left|k_{1 \varepsilon}-k_{\varepsilon}\right| \leq\left|p_{\varepsilon}\right|+\left|q_{\varepsilon}\right|+\left|r_{\varepsilon}\right|$, para todo $\varepsilon \in \mathbf{I}$, e $\left(l_{\varepsilon}\right)_{\varepsilon}$, $\left(m_{\varepsilon}\right)_{\varepsilon},\left(n_{\varepsilon}\right)_{\varepsilon},\left(p_{\varepsilon}\right)_{\varepsilon},\left(q_{\varepsilon}\right)_{\varepsilon},\left(r_{\varepsilon}\right)_{\varepsilon} \in \mathcal{N}(\mathbb{I R})$, temos que $\left(j_{1 \varepsilon}\right)_{\varepsilon}$ e $\left(k_{1 \varepsilon}\right)_{\varepsilon}$ são representantes de $j$ e $k$, respectivamente. Notemos que $z_{\varepsilon} \leq j_{1 \varepsilon}$ e $k_{1 \varepsilon} \leq w_{\varepsilon}$, para todo $\varepsilon \in \mathbf{I}$, o que implica que $z \leq j$ e $k \leq w$ 
Convém observarmos que, da demonstração da Proposição 1.2 .7 , se $x, y \in \overline{\mathbb{R}}$ e $\left(x_{\varepsilon}\right)_{\varepsilon}$ $\mathrm{e}\left(y_{\varepsilon}\right)_{\varepsilon}$ são representantes de $x$ e $y$, respectivamente, então

$$
x \vee y=\left[\left(\max \left\{x_{\varepsilon}, y_{\varepsilon}\right\}\right)_{\varepsilon}\right] \quad \text { e } \quad x \wedge y=\left[\left(\min \left\{x_{\varepsilon}, y_{\varepsilon}\right\}\right)_{\varepsilon}\right]
$$

Da Proposição 1.2 .7 e da Observação 1.2 .3 (c) e (d), temos que $(\overline{\mathbb{R}}, \geq)$ é um $\mathbb{\mathbb { R }}$-módulo reticulado e, assim, podemos definir

$$
x_{+}:=x \vee 0 \quad \text { e } \quad x_{-}:=(-x) \vee 0,
$$

e obtemos o seguinte:

Observação 1.2.8 Se $x \in \overline{\mathbb{R}} e\left(x_{\varepsilon}\right)_{\varepsilon}$ é um representante de $x$, então tem-se que

(a) o elemento $|x|$, definido na Definição 1.1.13, coincide com a definição usual de valor absoluto de $x$ tendo $(\overline{\mathbb{R}}, \geq)$ a estrutura de $\overline{\mathbb{R}}$-módulo reticulado, isto é,

$$
|x|:=\left[\left(\left|x_{\varepsilon}\right|\right)_{\varepsilon}\right]=\left[\left(\max \left\{x_{\varepsilon}, 0\right\}+\max \left\{-x_{\varepsilon}, 0\right\}\right)_{\varepsilon}\right]=x \vee 0+(-x) \vee 0=x_{+}+x_{-}
$$

(b) $x=\left[\left(x_{\varepsilon}\right)_{\varepsilon}\right]=\left[\left(\max \left\{x_{\varepsilon}, 0\right\}-\max \left\{-x_{\varepsilon}, 0\right\}\right)_{\varepsilon}\right]=x \vee 0-(-x) \vee 0=x_{+}-x_{-}$.

O próximo resultado, o qual surgiu durante a elabroração deste trabalho, nos fornece algumas propriedades envolvendo a relação de associação e a de ordem parcial sobre $\overline{\mathbb{R}}$.

Proposição 1.2.9 Sejam $x, y \in \overline{\mathbb{R}}$ e $r \in \mathbb{R}$. Tem-se que:

1. se $0 \leq x \leq y$ e $y \approx 0$, então $x \approx 0$;

2. se $0 \leq x \leq y$ e $y \alpha_{-r} \approx 0$, então $x \alpha_{-r} \approx 0$;

3. se $x \alpha_{-r} \approx 0$, então $|x| \leq \alpha_{r}$;

4. $x \alpha_{-r} \approx 0$ se, e somente se, $|x| \alpha_{-r} \approx 0$. 
Demonstração: Seja $\left(x_{\varepsilon}\right)_{\varepsilon}$ um representante de $x$.

1. Pela Proposição 1.2.6, existem $\left(x_{1 \varepsilon}\right)_{\varepsilon}$ e $\left(y_{1 \varepsilon}\right)_{\varepsilon}$ representantes de $x$ e $y$, respectivamente, tais que

$$
0 \leq x_{1 \varepsilon} \leq y_{1 \varepsilon}, \forall \varepsilon \in \mathbf{I}
$$

Como $\lim _{\varepsilon \downarrow 0} y_{1 \varepsilon}=0$, pois $y \approx 0$, segue que $\lim _{\varepsilon \downarrow 0} x_{1 \varepsilon}=0$, ou seja, $x \approx 0$.

2. Observemos que $\alpha_{-r} \geq 0$ e, assim, pela Observação $1.2 .3(\mathrm{~d})$, temos que $0 \leq x \alpha_{-r} \leq$ $y \alpha_{-r}$. Desse modo, por (1), concluímos que $x \alpha_{-r} \approx 0$.

3. Temos que $\lim _{\varepsilon \downarrow 0} \frac{x_{\varepsilon}}{\varepsilon^{r}}=0$. Então existe $\eta \in \mathbf{I}$ tal que $\left|x_{\varepsilon}\right| \leq \varepsilon^{r}$, para todo $\varepsilon \in \mathbf{I}_{\eta}$ e, portanto, $|x| \leq \alpha_{r}$.

4. Basta notarmos que

$$
\lim _{\varepsilon \downarrow 0} \frac{x_{\varepsilon}}{\varepsilon^{r}}=0 \Longleftrightarrow \lim _{\varepsilon \downarrow 0} \frac{\left|x_{\varepsilon}\right|}{\varepsilon^{r}}=0
$$

A seguir, apresentaremos algumas propriedades da função $|\cdot|: \overline{\mathbb{K}} \longrightarrow \overline{\mathbb{R}}$. As quatro primeiras afirmações nos induzem a chamar a função $|\cdot|$ de módulo generalizado sobre $\overline{\mathbb{K}}$.

Proposição 1.2.10 Sejam $x, y \in \bar{K}$. Tem-se que:

1. $|x| \geq 0 \quad$ e $\quad|-x|=|x|$;

2. $|x|=0$ se, e somente se, $x=0$;

3. $|x y|=|x||y|$;

4. $|x+y| \leq|x|+|y|$ e $\quad|| x|-| y|| \leq|x-y|$;

5. se $\mathbb{K}=\mathbb{R}$, tem-se que $|x|=x$ se, e somente se, $x \geq 0$; 
6. se $\mathbb{K}=\mathbb{R}$, tem-se que $|x|=-x$ se, e somente se, $x \leq 0$;

7. se $\mathbb{K}=\mathbb{R}$, então $|x| \geq x$ e $|x| \geq-x ;$

8. se $\mathbb{K}=\mathbb{R}, \quad x \leq y \quad$ e $-x \leq y$, então $|x| \leq y$.

Demonstração: Sejam $\left(x_{\varepsilon}\right)_{\varepsilon}$ e $\left(y_{\varepsilon}\right)_{\varepsilon}$ representantes de $x$ e $y$, respectivamente.

1. Basta observarmos que $|x|=\left[\left(\left|x_{\varepsilon}\right|\right)_{\varepsilon}\right],-x=\left[\left(-x_{\varepsilon}\right)_{\varepsilon}\right]$ e $|-x|=\left[\left(\left|-x_{\varepsilon}\right|\right)_{\varepsilon}\right]$.

2. Segue diretamente da definição de elemento nulo.

3. Basta observarmos que $|x y|=\left[\left(\left|x_{\varepsilon} y_{\varepsilon}\right|\right)_{\varepsilon}\right]=\left[\left(\left|x_{\varepsilon}\right|\left|y_{\varepsilon}\right|\right)_{\varepsilon}\right]=\left[\left(\left|x_{\varepsilon}\right|\right)_{\varepsilon}\right]\left[\left(\left|y_{\varepsilon}\right|\right)_{\varepsilon}\right]=|x||y|$.

4. É suficiente notarmos que $|x+y|=\left[\left(\left|x_{\varepsilon}+y_{\varepsilon}\right|\right)_{\varepsilon}\right],|x|=\left[\left(\left|x_{\varepsilon}\right|\right)_{\varepsilon}\right],|y|=\left[\left(\left|y_{\varepsilon}\right|\right)_{\varepsilon}\right]$, ||$x|-| y||=\left[\left(|| x_{\varepsilon}|-| y_{\varepsilon}||\right)_{\varepsilon}\right],|x-y|=\left[\left(\left|x_{\varepsilon}-y_{\varepsilon}\right|\right)_{\varepsilon}\right]$ e que

$$
\left|x_{\varepsilon}+y_{\varepsilon}\right| \leq\left|x_{\varepsilon}\right|+\left|y_{\varepsilon}\right| \quad \text { e } \quad|| x_{\varepsilon}|-| y_{\varepsilon}|| \leq\left|x_{\varepsilon}-y_{\varepsilon}\right|, \forall \varepsilon \in \mathbf{I} \text {. }
$$

5. Basta usarmos 1. e notarmos que, se $x \geq 0$, então existe $\left(x_{1 \varepsilon}\right)_{\varepsilon}$ representante de $x$ tal que $x_{1 \varepsilon} \geq 0$, para todo $\varepsilon \in \mathbf{I}$, e usarmos que

$$
x=\left[\left(x_{1 \varepsilon}\right)_{\varepsilon}\right]=\left[\left(\left|x_{1 \varepsilon}\right|\right)_{\varepsilon}\right]=|x|
$$

6. É suficiente observarmos que, de 1. e 5., temos que

$$
x \leq 0 \Longleftrightarrow-x \geq 0 \Longleftrightarrow|-x|=-x \Longleftrightarrow|x|=-x .
$$

7. Decorre de $\left|x_{\varepsilon}\right| \geq x_{\varepsilon}$ e $\left|x_{\varepsilon}\right| \geq-x_{\varepsilon}$, para todo $\varepsilon \in \mathbb{I}$.

8. Por hipótese, dado $b>0$ existem $\eta_{1}, \eta_{2} \in \mathbf{I}$ tais que

$$
y_{\varepsilon}-x_{\varepsilon} \geq-\varepsilon^{b}, \quad \forall \varepsilon \in \mathbf{I}_{\eta_{1}}, \quad \text { e } \quad y_{\varepsilon}+x_{\varepsilon} \geq-\varepsilon^{b}, \quad \forall \varepsilon \in \mathbf{I}_{\eta_{2}} .
$$


Seja $\eta=\min \left\{\eta_{1}, \eta_{2}\right\}$. Logo,

$$
y_{\varepsilon}-x_{\varepsilon} \geq-\varepsilon^{b} \quad \text { e } \quad y_{\varepsilon}+x_{\varepsilon} \geq-\varepsilon^{b}, \quad \forall \varepsilon \in \mathbf{I}_{\eta} .
$$

Assim, $y_{\varepsilon}-\left|x_{\varepsilon}\right| \geq-\varepsilon^{b}$, para todo $\varepsilon \in \mathbf{I}_{\eta}$. Portanto, $y-|x| \geq 0$, ou seja, $y \geq|x|$.

\subsection{Uma ultramétrica para $\bar{K}^{n}$}

Nesta seção, apresentaremos a topologia cortante sobre $\overline{\mathbb{K}}$ desenvolvida por D. Scarpalezos. A partir dessa topologia, munimos $\overline{\mathbb{K}}^{n}$ com a topologia produto. Essas serão as topologias utilizadas, para os nossos objetivos nos demais capítulos, sobre $\overline{\mathbb{K}}$ e sobre $\overline{\mathbb{K}}^{n}$, respectivamente.

Em seu trabalho, D. Scarpalezos, para cada $\left(x_{\varepsilon}\right)_{\varepsilon} \in \mathcal{E}_{M}(\mathbb{K})$, define

$$
\begin{gathered}
S\left(\left(x_{\varepsilon}\right)_{\varepsilon}\right):=\left\{a \in \mathbb{R}:\left|x_{\varepsilon}\right|=o\left(\varepsilon^{a}\right), \text { quando } \varepsilon \downarrow 0\right\} ; \\
V\left(\left(x_{\varepsilon}\right)_{\varepsilon}\right):=\left\{\begin{array}{ll}
\sup S\left(\left(x_{\varepsilon}\right)_{\varepsilon}\right) & , \text { se } S\left(\left(x_{\varepsilon}\right)_{\varepsilon}\right) \text { é limitado superiormente } \\
+\infty & , \text { caso contrário }
\end{array} ;\right.
\end{gathered}
$$

e obteve que a função $d: \overline{\mathbb{K}} \times \overline{\mathbb{K}} \longrightarrow \mathbb{R}_{+}$, dada por

$$
d\left(\left[\left(x_{\varepsilon}\right)_{\varepsilon}\right],\left[\left(y_{\varepsilon}\right)_{\varepsilon}\right]\right):= \begin{cases}e^{-V\left(\left(x_{\varepsilon}-y_{\varepsilon}\right)_{\varepsilon}\right)} & , \text { se } V\left(\left(x_{\varepsilon}-y_{\varepsilon}\right)_{\varepsilon}\right) \in \mathbb{R} \\ 0 & , \text { se } V\left(\left(x_{\varepsilon}-y_{\varepsilon}\right)_{\varepsilon}\right)=+\infty\end{cases}
$$

é uma ultramétrica sobre $\overline{\mathbb{K}}$ e que $\overline{\mathbb{K}}$ munido com a topologia induzida por essa ultramétrica é um espaço topológico completo.

Recentemente, J. Aragona, R. Fernandez e S. O. Juriaans ([AFJ-3]) observaram que a função $d$ poderia ser apresentada sem fazer referência aos representantes dos elementos de $\overline{\mathbb{K}}$, utilizando, para isso, a relação de associação em $\bar{K}$. Essa nova abordagem, por ter a vantagem de independer dos representantes, pela simplicidade de notação e por facilitar as demonstrações, será a utilizada neste trabalho. 
Lembramos que, se $\rho$ é uma métrica sobre um conjunto $\mathrm{M}$ tal que

$$
\rho(a, b) \leq \max \{\rho(a, c), \rho(c, b)\}, \quad \forall a, b, c \in M
$$

então $\rho$ é chamada de ultramétrica e o espaço $(M, \rho)$ é chamado de espaço ultramétrico.

Definição 1.3.1 Define-se, para cada $x \in \overline{\mathbb{K}}$,

$$
A(x):=\left\{r \in \mathbb{R}: x \alpha_{-r} \approx 0\right\}
$$

Convém observarmos que, se $x \in \bar{K}$, temos, da definição de $\mathcal{E}_{M}(\mathbb{I})$, que $A(x) \neq \varnothing$.

Notamos que, se $\left(x_{\varepsilon}\right)_{\varepsilon}$ é um representante de $x$ e $r \in \mathbb{R}$, então

$$
\left|x_{\varepsilon}\right|=o\left(\varepsilon^{r}\right), \text { quando } \varepsilon \downarrow 0 \Longleftrightarrow \lim _{\varepsilon \downarrow 0} \frac{\left|x_{\varepsilon}\right|}{\varepsilon^{r}}=0 \Longleftrightarrow \lim _{\varepsilon \downarrow 0} \frac{x_{\varepsilon}}{\varepsilon^{r}}=0 \Longleftrightarrow x \alpha_{-r} \approx 0 .
$$

Logo, para todo $\left(x_{\varepsilon}\right)_{\varepsilon}$ representante de $x$, tem-se que $S\left(\left(x_{\varepsilon}\right)_{\varepsilon}\right)=A(x)$ e, se $A(x)$ é limitado superiormente, então $V\left(\left(x_{\varepsilon}\right)_{\varepsilon}\right):=\sup A(x)$.

Definição 1.3.2 Define-se, para cada $x \in \bar{K}$,

$$
\mathcal{V}(x):=\left\{\begin{array}{ll}
\sup A(x) & , \text { se } A(x) \text { é limitado superiormente } \\
+\infty & , \text { caso contrário }
\end{array} .\right.
$$

Apresentamos, a seguir, algumas propriedades do conjunto $A(x)$.

Proposição 1.3.3 Seja $x \in \overline{\mathbb{K}}$. As seguintes afirmações são válidas:

1. se $a, b \in \mathbb{R}, a \in A(x)$ e $b<a$, então $b \in A(x)$;

2. $A(x)=\mathbb{R}$, ou existe $r \in \mathbb{R}$ tal que $A(x)=]-\infty, r[$ ou $A(x)=]-\infty, r]$. 
Demonstração: A afirmação 1. segue da Proposição 1.1 .12 (5) e a afirmação 2. segue de $1 .$.

Destacamos que $\mathcal{V}(\cdot)$ tem as seguintes propriedades:

Proposição 1.3.4 Sejam $x \in \bar{K}$ e $c \in \mathbb{R}$. Tem-se que:

1. $\mathcal{V}(x)=+\infty$ se, e somente se, $A(x)=\mathbb{R}$;

2. $\mathcal{V}(x)=+\infty$ se, e somente se, $x=0$;

3. se $\mathcal{V}(x)=+\infty$, ou se $c<\mathcal{V}(x)<+\infty$, então $c \in A(x)$.

Demonstração: A primeira afirmação segue da Proposição 1.3.3 (2). A segunda decorre de 1. e da Proposição 1.1.12 (4). Para provarmos a última afirmação, basta observarmos que, se $\mathcal{V}(x)=+\infty$, então, por $1 ., A(x)=\mathbb{R}$ e, portanto, $c \in A(x)$, e se $\mathcal{V}(x) \in \mathbb{R}$ basta usarmos a definição de sup e a Proposição 1.3.3 (1).

Definição 1.3.5 Define-se $\|\cdot\|: \overline{\mathbb{K}} \longrightarrow \mathbb{R}_{+}$e $D: \overline{\mathbb{K}} \times \overline{\mathbb{K}} \longrightarrow \mathbb{R}_{+}$por

$$
\|x\|:=\left\{\begin{array}{ll}
e^{-\mathcal{V}(x)} & , \text { se } \mathcal{V}(x) \in \mathbb{R} \\
0 & , \text { se } \mathcal{V}(x)=+\infty
\end{array} \quad \text { e } \quad D(x, y):=\|x-y\| .\right.
$$

Observamos que, pelos comentários anteriores, a função $D$ coincide com a função $d$ definida por D. Scarpalezos. Resultados relativos a $d$ (e, portanto, a $D$ ) e a $\|\cdot\|$, com suas respectivas provas, podem ser encontrados, por exemplo, em [AJ] e [Sil-A]. Contudo, para mostrarmos que com essa nova abordagem as demostrações ficam mais simples, provaremos as propriedades da função $\|\cdot\|$ - as quais serão bastante utilizadas nesta Dissertação - e que $D$ é uma ultramétrica, invariante por translações, sobre $\overline{\mathbb{K}}$. Destacamos, ainda, que no decorrer de nossos estudos, surgiram mais algumas propriedades da função || || (Proposição 1.3.7 (9), (10) e (11)) as quais são interessantes e pertinentes a este trabalho. 
Convém notarmos também que $\|\cdot\|$ não é uma norma (Proposição 1.3 .7 (2)). No entanto, em virtude das afirmações (1), (2), (3) e (4) da Proposição 1.3.7, a notação usual de norma será mantida.

Em razão da definição de $\|\cdot\|$ utilizar $\mathcal{V}(\cdot)$ é importante observarmos o seguinte fato:

Lema 1.3.6 Sejam $x, y \in \overline{\mathbb{K}}$. Se $\mathcal{V}(x)=+\infty$, ou se $\mathcal{V}(y) \leq \mathcal{V}(x)<+\infty$, então $\|x\| \leq\|y\|$.

Demonstração: Se $\mathcal{V}(x)=+\infty$, então, pela Definição 1.3.5, $\|x\|=0 \leq\|y\|$. Por outro lado, se $\mathcal{V}(x) \in \mathbb{R}$, temos que $-\mathcal{V}(x) \leq-\mathcal{V}(y)$ e, assim, $\|x\|=e^{-\mathcal{V}(x)} \leq e^{-\mathcal{V}(y)}=\|y\|$.

Proposição 1.3.7 Sejam $x, y \in \overline{\mathbb{K}}, r \in \mathbb{R}$ e $a, b \in \mathbb{K}$. Tem-se que:

1. $\|x+y\| \leq \max \{\|x\|,\|y\|\}$

2. $\|x y\| \leq\|x\|\|y\|$;

3. $\|x\|=0$ se, $e$ somente se, $x=0$;

4. $\|a x\|=\|x\|$, se $a \neq 0$;

5. $\left\|\alpha_{r} x\right\|=e^{-r}\|x\| ;$

6. $\left\|\left[\left(a_{\varepsilon}\right)_{\varepsilon}\right]\right\|=1$, se $a \neq 0$, onde $a_{\varepsilon}:=a$, para todo $\varepsilon \in \mathbf{I}$;

7. $\left\|\left[\left(a_{\varepsilon}-b_{\varepsilon}\right)_{\varepsilon}\right]\right\|=1-\delta_{i, j}$, onde $a_{\varepsilon}:=a, b_{\varepsilon}:=b$, para todo $\varepsilon \in \mathbf{I}$, e $\delta_{i, j}:=1$, se $i=j$, e $\delta_{i, j}:=0$, se $i \neq j$;

8. $\left\|\alpha_{r}\right\|=e^{-r}$

9. $\|x\|=\||x|\|$;

10. se $|x| \leq|y|$, então $\|x\| \leq\|y\|$; 
11. $|\|x\|-\|y\|| \leq\|x-y\|$.

Demonstração: 1. Pela Proposição 1.3 .3 (2), temos que $A(x) \subset A(y)$ ou $A(y) \subset A(x)$. Suponhamos, sem perda de generalidade, que $A(y) \subset A(x)$. Então $\mathcal{V}(x)=+\infty$ ou $\mathcal{V}(y) \leq$ $\mathcal{V}(x)<+\infty$ e assim, pelo Lema 1.3.6, $\|x\| \leq\|y\|$.

Seja $s \in \mathbb{R}$ tal que $s \in A(y) \subset A(x)$. Logo, $y \alpha_{-s} \approx 0$ e $x \alpha_{-s} \approx 0$ e, pela Proposição $1.1 .12(7)$, temos que $(x+y) \alpha_{-s} \approx 0$ e assim $s \in A(x+y)$. Desse modo, obtemos que $A(y) \subset A(x+y)$ e, conseqüentemente, $\mathcal{V}(x+y)=+\infty$ ou $\mathcal{V}(y) \leq \mathcal{V}(x+y)<+\infty$. Portanto, pelo Lema 1.3.6, concluímos que $\|x+y\| \leq\|y\|=\max \{\|x\|,\|y\|\}$.

2. Primeiramente, para facilitar a escrita, convencionamos que o símbolo $+\infty$ é estritamente maior que qualquer número real. Sejam $r, s \in \mathbb{R}$ tais que $r<\mathcal{V}(x)$ e $s<\mathcal{V}(y)$. Pela Proposição 1.3.4 (3), temos que $r \in A(x)$ e $s \in A(y)$ e, portanto, $x \alpha_{-r} \approx 0$ e $y \alpha_{-s} \approx 0$. Assim, pela Proposição $1.1 .12(8)$, segue que $x y \alpha_{-(r+s)} \approx 0$. Logo, $r+s \in A(x y)$, o que implica que $\mathcal{V}(x y) \geq r+s$.

Suponhamos que $\mathcal{V}(x), \mathcal{V}(y) \in \mathbb{R}$. Como $r, s \in \mathbb{R}$ foram escolhidos arbitrariarmente temos, em particular, para $r=\mathcal{V}(x)-\frac{\sigma}{2}$ e $s=\mathcal{V}(y)-\frac{\sigma}{2}$, onde $\sigma>0$, que

$$
\mathcal{V}(x y) \geq \mathcal{V}(x)-\frac{\sigma}{2}+\mathcal{V}(y)-\frac{\sigma}{2}=\mathcal{V}(x)+\mathcal{V}(y)-\sigma
$$

$\log$,

$$
\mathcal{V}(x y)=+\infty \quad \text { ou } \quad \mathcal{V}(x y)=\lim _{\sigma \downarrow 0} \mathcal{V}(x y) \geq \lim _{\sigma \downarrow 0}(\mathcal{V}(x)+\mathcal{V}(y)-\sigma)=\mathcal{V}(x)+\mathcal{V}(y)
$$

Desse modo, se $\mathcal{V}(x y) \in \mathbb{R}$, então $\|x y\|=e^{-\mathcal{V}(x y)} \leq e^{-\mathcal{V}(x)-\mathcal{V}(y)}=e^{-\mathcal{V}(x)} e^{-\mathcal{V}(y)}=\|x\|\|y\|$. Por outro lado, se $\mathcal{V}(x y)=+\infty$, então, pela Definição $1.3 .5,\|x y\|=0$ e portanto obtemos que $\|x y\| \leq\|x\|\|y\|$, nesse caso.

Analisaremos, a seguir, o caso em que $\mathcal{V}(x)=+\infty$ ou $\mathcal{V}(y)=+\infty$. Suponhamos, sem perda de generalidade, que $\mathcal{V}(x)=+\infty$. Então $\mathcal{V}(x y) \geq r+s$, para todo $r \in \mathbb{R}$ e todo $s<\mathcal{V}(y)$. Assim, $\mathcal{V}(x y)=+\infty$, pois $\lim _{r \rightarrow+\infty}(r+s)=+\infty$. Portanto, $\|x y\|=0$ e conseqüentemente $\|x y\| \leq\|x\|\|y\|$, também nesse caso. 
3. Segue da Proposição 1.3.4 (2) e da Definição 1.3.5.

4. Pela Proposição 1.1.12 (1), para $r \in \mathbb{R}$, temos que $x \alpha_{-r} \approx 0$ se, e somente se, $a x \alpha_{-r} \approx 0$. Dessa forma, $A(x)=A(a x)$ e portanto $\mathcal{V}(x)=\mathcal{V}(a x)$. Logo, $\|x\|=\|a x\|$.

5. Seja $b \in \mathbb{R}$. Temos que

$$
b \in A\left(\alpha_{r} x\right) \Longleftrightarrow \alpha_{r} x \alpha_{-b} \approx 0 \Longleftrightarrow x \alpha_{-(b-r)} \approx 0 \Longleftrightarrow b-r \in A(x)
$$

Logo, $b \in A\left(\alpha_{r} x\right)$ se, e somente se, $b \in r+A(x)$, e portanto $A\left(\alpha_{r} x\right)=r+A(x)$.

Suponhamos que $A(x)$ seja limitado superiormente, ou seja, $A(x) \neq \mathbb{R}$ (Proposição 1.3.3 (2)). Logo, $A\left(\alpha_{r} x\right)$ é limitado superiormente e, portanto,

$$
\mathcal{V}\left(\alpha_{r} x\right)=\sup A\left(\alpha_{r} x\right)=\sup (r+A(x))=r+\sup A(x)=r+\mathcal{V}(x)
$$

Desse modo, concluímos que $\left\|\alpha_{r} x\right\|=e^{-\mathcal{V}\left(\alpha_{r} x\right)}=e^{-r-\mathcal{V}(x)}=e^{-r} e^{-\mathcal{V}(x)}=e^{-r}\|x\|$.

Se $A(x)=\mathbb{R}$, então $A\left(\alpha_{r} x\right)=\mathbb{R}$. Assim, $\mathcal{V}(x)=+\infty=\mathcal{V}\left(\alpha_{r} x\right)$ e portanto $\|x\|=$ $0=\left\|\alpha_{r} x\right\|$. Logo, obtemos que $\left\|\alpha_{r} x\right\|=e^{-r}\|x\|$.

6. Pela Proposição 1.1.12 (6), temos que $\left[\left(a_{\varepsilon}\right)_{\varepsilon}\right] \alpha_{-r} \approx 0$ se, e somente se, $r<0$. Portanto, $\left.A\left(\left[\left(a_{\varepsilon}\right)_{\varepsilon}\right]\right)=\right]-\infty, 0\left[\right.$, o que implica que $\mathcal{V}\left(\left[\left(a_{\varepsilon}\right)_{\varepsilon}\right]\right)=0$, de onde resulta que $\left\|\left[\left(a_{\varepsilon}\right)_{\varepsilon}\right]\right\|=e^{-\mathcal{V}\left(\left[\left(a_{\varepsilon}\right)_{\varepsilon}\right]\right)}=1$.

7. Conseqüência de 3 . e 6 ..

8. Segue diretamente de 5 . e 6 , considerando $a_{\varepsilon}:=1$, para todo $\varepsilon \in \mathbf{I}$.

9. Seja $r \in \mathbb{R}$ e observemos que, pela Proposição 1.2.9 (4),

$$
x \alpha_{-r} \approx 0 \Longleftrightarrow|x| \alpha_{-r} \approx 0
$$

Logo, $A(x)=A(|x|)$, o que implica que $\mathcal{V}(x)=\mathcal{V}(|x|)$, e conseqüentemente $\|x\|=\||x|\|$. 
10. Consideremos $r \in \mathbb{R}$ e suponhamos que $|y| \alpha_{-r} \approx 0$. Temos, por hipótese, que $0 \leq|x| \leq|y|$. Assim, pela Proposição 1.2 .9 (2), segue que $|x| \alpha_{-r} \approx 0$. Portanto, $A(|y|) \subset$ $A(|x|)$, o que implica que $\mathcal{V}(|x|)=+\infty$ ou $\mathcal{V}(|y|) \leq \mathcal{V}(|x|)<+\infty$. Logo, pelo Lema 1.3.6, obtemos que $\||x|\| \leq\||y|\|$ e assim, por 9., concluímos que $\|x\| \leq\|y\|$.

11. De 1. e 4., temos que

$$
\|x\|=\|(x-y)+y\| \leq \max \{\|x-y\|,\|y\|\} \leq\|x-y\|+\|y\|
$$

$\|y\|=\|(y-x)+x\| \leq \max \{\|(-1)(x-y)\|,\|x\|\}=\max \{\|x-y\|,\|x\|\} \leq\|x-y\|+\|x\| ;$

e, portanto, $\|x-y\| \geq|\|x\|-\|y\||$.

Teorema 1.3.8 A função $D$, definida na Definição 1.3.5, é uma ultramétrica sobre $\overline{\mathbb{K}}$. Além disso, $D$ é invariante por translações, isto é, $D(x, y)=D(x+z, y+z)$, para todos $x, y, z \in \bar{K}$.

Demonstração: Sejam $x, y, z \in \bar{K}$.

Notemos que, da Definição 1.3.5, segue que

$$
D(x, y)=\|x-y\|=\|(x+z)-(y+z)\|=D(x+z, y+z) .
$$

Pela Proposição 1.3.7 (1), (3) e (4), obtemos que

$$
\begin{gathered}
\|x-y\|=0 \Longleftrightarrow x-y=0 \Longleftrightarrow x=y ; \quad \quad\|x-y\|=\|(-1)(y-x)\|=\|y-x\| ; \\
\|x-y\|=\|(x-z)+(z-y)\| \leq \max \{\|x-z\|,\|z-y\|\} .
\end{gathered}
$$

Portanto, $D$ é uma ultramétrica.

Definição 1.3.9 A estrutura uniforme gerada por $D$ sobre $\overline{\mathbb{K}}$ é chamada de estrutura uniforme cortante de $\overline{\mathbb{I}}$ e a topologia induzida por $D$ é chamada de topologia cortante de $\overline{\mathbb{K}}$ e é denotada por $\mathscr{T}_{c}$. 
Enunciamos, a seguir, alguns resultados topológicos do espaço ultramétrico $\left(\bar{K}, \mathscr{T}_{c}\right)$. Esses resultados foram desenvolvidos por D. Scarpalezos ([Sca-1], [Sca-2]) e por J. Aragona e S. O. Juriaans ([AJ]). Pode-se também encontrar uma prova detalhada de todos esses resultados em [Sil-A].

- $\left(\overline{\mathbb{I K}}, \mathscr{T}_{c}\right)$ é completo;

- $\left(\bar{K}, \mathscr{T}_{c}\right)$ é um anel topológico;

- $\left(\overline{\mathbb{I}}, \mathscr{T}_{c}\right)$ não é um $\mathbb{K}$-espaço vetorial topológico;

- $\left(\overline{\mathbb{I}}, \mathscr{T}_{c}\right)$ não é separável;

- $\left(\overline{\mathbb{I}}, \mathscr{T}_{c}\right)$ não é localmente compacto.

Definição 1.3.10 Para $n \in \mathbb{N}^{*}$, define-se $\|\cdot\|_{n}: \overline{\mathbb{K}}^{n} \longrightarrow \mathbb{R}_{+}$e $D_{n}: \overline{\mathbb{K}}^{n} \times \overline{\mathbb{K}}^{n} \longrightarrow \mathbb{R}_{+}$ por

$$
\begin{aligned}
\|x\|_{n} & :=\max \left\{\left\|x_{i}\right\|: 1 \leq i \leq n\right\}, \text { onde } x=\left(x_{1}, \ldots, x_{n}\right) ; \\
D_{n}(x, y) & :=\|x-y\|_{n} .
\end{aligned}
$$

A fim de simplificarmos a notação, denotaremos $\|\cdot\|_{n}$ por $\|\cdot\|$, a menos de menção em contrário.

Observamos que $D_{n}$ é uma ultramétrica para $\overline{\mathbb{K}}^{n}$. Dessa forma, $\overline{\mathbb{K}}^{n}$ munido com a topologia produto, a qual denotaremos por $\mathscr{T}_{c}^{n}$, é um espaço ultramétrico.

\subsection{Algumas propriedades de $\overline{\mathbb{K}}^{n}$}


As definições e a maioria dos resultados apresentados nesta seção fazem parte dos artigos [AJ], [AFJ-1] e [AJOS]. Ressaltamos que, a partir do estudo da prova de alguns dos resultados encontrados em [AJ], nos foi possível obter a Proposição 1.4.6 (3), a Proposição 1.4.7 (3) e a Proposição 1.4.8.

A seguir, destacamos um resultado válido em espaços ultramétricos completos $(M, \rho)$ $\operatorname{com} \rho$ invariante por translações, portanto verdadeiro em $\left(\overline{\mathbb{K}}, \mathscr{T}_{c}\right)$, que é interessante e será utilizado posteriormente. Para prová-lo necessitamos do seguinte Lema:

Lema 1.4.1 Seja $(M, \rho)$ um espaço ultramétrico e $\left(x_{n}\right)_{n \in N}$ uma seqüência de elementos de $M$. Então $\left(x_{n}\right)_{n \in N}$ é uma seqüência de Cauchy em relação a $\rho$ se, e somente se, dado $\varepsilon \geq 0$, existe $N \in \mathbb{N}$ tal que $\rho\left(x_{n+1}, x_{n}\right) \leq \varepsilon$, para todo $n \geq N$.

Demonstração: Ver [Sil-A, Capítulo 2].

Proposição 1.4.2 Seja $(M, \rho)$ um espaço ultramétrico completo com $\rho$ invariante por translações. Se $\left(x_{n}\right)_{n \in \mathbb{N}}$ é uma seqüência de elementos dê $M$, então a série $\sum_{n=0}^{\infty} x_{n}$ converge se, e somente se, $\lim _{n \rightarrow \infty} \rho\left(x_{n}, 0\right)=0$.

Demonstração: Seja, para cada $n \in \mathbb{N}, S_{n}:=\sum_{j=0}^{n} x_{j}$. Suponhamos que exista $a \in \bar{K}$ tal que $a=\lim _{n \rightarrow \infty} S_{n}$. Então

$$
\lim _{n \rightarrow \infty} x_{n}=\lim _{n \rightarrow \infty}\left(S_{n}-S_{n-1}\right)=\lim _{n \rightarrow \infty} S_{n}-\lim _{n \rightarrow \infty} S_{n-1}=a-a=0
$$

ou seja, $\lim _{n \rightarrow \infty} \rho\left(x_{n}, 0\right)=0$.

Reciprocamente, suponhamos que $\lim _{n \rightarrow \infty} \rho\left(x_{n}, 0\right)=0$. Como $\rho$ é invariante por translações, temos que

$$
\rho\left(S_{n+1}, S_{n}\right)=\rho\left(S_{n+1}-S_{n}, 0\right)=\rho\left(x_{n+1}, 0\right),
$$

$\operatorname{logo}$

$$
\lim _{n \rightarrow \infty} \rho\left(S_{n+1}, S_{n}\right)=\lim _{n \rightarrow \infty} \rho\left(x_{n+1}, 0\right)=0 .
$$


Assim, pelo Lema 1.4.1, temos que a seqüência $\left(S_{n}\right)_{n \in N}$ é de Cauchy e, portanto, como $M$ é completo, segue que $\left(S_{n}\right)_{n \in N}$ é convergente em $(M, \rho)$.

Do próximo resultado e da Proposição 1.4.2, obtemos condições necessárias e suficientes para que uma série, de elementos de $\overline{\mathbb{K}}$, seja convergente.

Proposição 1.4.3 Seja $\left(x_{n}\right)_{n \in N}$ uma seqüência de elementos de $\bar{K}$. São equivalentes:

1. $\lim _{n \rightarrow \infty}\left\|x_{n}\right\|=0$;

2. para todo $p \in \mathbb{N}$, existe $N_{p} \in \mathbb{N}$ tal que $x_{n} \alpha_{-p} \approx 0$, para todo $n \geq N_{p}$.

Demonstração: Suponhamos 1. verdadeira e seja $p \in \mathbb{N}$. Então existe $N_{p} \in \mathbb{N}$ tal que $\left\|x_{n}\right\|<e^{-p}$, para todo $n \geq N_{p}$.

Da Definição 1.3 .5 e da Proposição 1.3.4 (3), temos, para todo $n \geq N_{p}$, que

$$
\begin{aligned}
\left\|x_{n}\right\|<e^{-p} & \Longrightarrow\left\|x_{n}\right\|=0 \quad \text { ou } \quad 0<\left\|x_{n}\right\|<e^{-p} \\
& \Longrightarrow \mathcal{V}\left(x_{n}\right)=+\infty \quad \text { ou } \quad p<\mathcal{V}\left(x_{n}\right)<+\infty \\
& \Longrightarrow p \in A\left(x_{n}\right) \\
& \Longrightarrow x_{n} \alpha_{-p} \approx 0 .
\end{aligned}
$$

Portanto, 2. é verdadeira.

Reciprocamente, seja $\sigma>0$. Então, para $p \in \mathbb{N} \operatorname{com} e^{-p}<\sigma$, existe $N_{p} \in \mathbb{N}$ tal que $x_{n} \alpha_{-p} \approx 0$, para todo $n \geq N_{p}$.

Da Definição 1.3.5 e da Proposição 1.3.3 (2), temos, para todo $n \geq N_{p}$, que

$$
\begin{aligned}
x_{n} \alpha_{-p} \approx 0 & \Longrightarrow p \in A\left(x_{n}\right) \\
& \Longrightarrow]-\infty, p] \subset A\left(x_{n}\right) \\
& \Longrightarrow \mathcal{V}\left(x_{n}\right)=+\infty \quad \text { ou } \quad p \leq \mathcal{V}\left(x_{n}\right)<+\infty \\
& \Longrightarrow\left\|x_{n}\right\|=0 \quad \text { ou } \quad 0<\left\|x_{n}\right\| \leq e^{-p}<\sigma \\
& \Longrightarrow\left\|x_{n}\right\|<\sigma
\end{aligned}
$$


e $\operatorname{assim} 1$. é verdadeira.

A partir do conceito de associação em $\overline{\mathbb{K}}$ (Definição 1.1.10) define-se a associação em $\overline{\mathbb{K}}^{n}$. Esse conceito é apresentado a seguir.

Definição 1.4.4 Sejam $x=\left(x_{1}, \ldots, x_{n}\right), y=\left(y_{1}, \ldots, y_{n}\right) \in \overline{\mathbb{K}}^{n}$. Diz-se que $x$ é associado a y em $\overline{\mathbb{K}}^{n}$, e denota-se por $x \approx y$, se, e somente se, $x_{i}$ é associado a $y_{i}$ em $\overline{\mathbb{K}}$, para todo $1 \leq i \leq n$.

Da Definição 1.4.4, temos que, se $x=\left(\left[\left(x_{1 \varepsilon}\right)_{\varepsilon}\right], \ldots,\left[\left(x_{n \varepsilon}\right)_{\varepsilon}\right]\right), y=\left(\left[\left(y_{1 \varepsilon}\right)_{\varepsilon}\right], \ldots,\left[\left(y_{n \varepsilon}\right)_{\varepsilon}\right]\right)$ $\in \overline{\mathbb{K}}^{n},\left(x_{\varepsilon}\right)_{\varepsilon}:=\left(\left(x_{1 \varepsilon}, \ldots, x_{n \varepsilon}\right)\right)_{\varepsilon},\left(y_{\varepsilon}\right)_{\varepsilon}:=\left(\left(y_{1 \varepsilon}, \ldots, y_{n \varepsilon}\right)\right)_{\varepsilon}$ e $x \approx y$, então

$$
\lim _{\varepsilon \downarrow 0}\left|x_{\varepsilon}-y_{\varepsilon}\right|=\lim _{\varepsilon \downarrow 0}\left(\sum_{j=1}^{n}\left|x_{j \varepsilon}-y_{j \varepsilon}\right|^{2}\right)^{\frac{1}{2}}=0 .
$$

Proposição 1.4.5 Seja $x=\left(x_{1}, \ldots, x_{n}\right) \in \overline{\mathbb{K}}^{n}$. As seguintes afirmações são válidas:

1. se $x \in B_{1}(0)$, então tem-se que

(a) $x \approx 0$;

(b) $\|1-x\| \geq 1$;

2. $1 \notin \overline{B_{1}(0)}, B_{1}(0) \cap B_{1}(1)=\varnothing, B_{1}^{\prime}(0) \supset \overline{B_{1}(0)}$ e $B_{1}^{\prime}(0) \neq \overline{B_{1}(0)}$.

Demonstração: Observemos, primeiramente, que

$$
\begin{aligned}
x \in B_{1}(0) & \Longleftrightarrow\|x\|_{n}=\max \left\{\left\|x_{i}\right\|: 1 \leq i \leq n\right\}<1 \\
& \Longleftrightarrow \forall 1 \leq i \leq n, \text { tem-se que } \mathcal{V}\left(x_{i}\right)=+\infty \text { ou } 0<\mathcal{V}\left(x_{i}\right)<+\infty
\end{aligned}
$$

Seja $x \in B_{1}(0)$.

Provaremos, a seguir, a afirmação $1 .$. 
Por (1.3) e pela Proposição 1.3.4 (3), temos que $x_{i}=x_{i} \alpha_{-0} \approx 0$, para todo $1 \leq i \leq n$, e, assim, pela Definição 1.4.4, obtemos que $x \approx 0$, o que prova 1.(a).

Para provarmos 1.(b), basta notarmos que $x \approx 0$ implica $1-x \approx 1$ e portanto, por 1.(a), temos que $1-x \notin B_{1}(0)$, ou seja, $\|1-x\| \geq 1$.

Finalmente, provaremos 2 . É claro que $\overline{B_{1}(0)} \subset B_{1}^{\prime}(0)$.

Suponhamos que $1 \in \overline{B_{1}(0)}$. Então existe uma seqüência $\left(x_{m}\right)_{m \in N}$ de elementos de $B_{1}(0)$ tal que

$$
\lim _{m \rightarrow \infty}\left\|1-x_{m}\right\|=0,
$$

o que contradiz 1.(b). Portanto, $1 \notin \overline{B_{1}(0)}$.

Por 1.(b), temos que, se $x \in B_{1}(0)$, então $\|1-x\| \geq 1$ e, portanto, $x \notin B_{1}(1)$. Logo, $B_{1}(0) \cap B_{1}(1)=\varnothing$. Pela proposição $1.3 .7(6)$, temos que $\|1\|=1 \mathrm{e}$, portanto, $1 \in B_{1}^{\prime}(0)$. Dessa forma, concluímos que $\overline{B_{1}(0)} \subset B_{1}^{\prime}(0)$ e $\overline{B_{1}(0)} \neq B_{1}^{\prime}(0)$.

Na Proposição 1.4.7, será provado que o conjunto dos elementos invertíveis de $\overline{\mathbb{K}}$ é um subconjunto aberto de $\bar{K}$. Para tanto, precisamos do próximo resultado.

Proposição 1.4.6 Sejam $\left(a_{n}\right)_{n \in \boldsymbol{N}}$ uma seqüência de elementos de $\mathbb{K}$ e $x \in \overline{\mathbb{I}}$ com $\|x\|<1$. As seguintes afirmaçôes são verdadeiras:

1. $\sum_{n=0}^{\infty} a_{n} x^{n}$ converge em $\overline{\mathbb{K}}$ e, em particular, $\sum_{n=0}^{\infty} x^{n}$ converge;

2. $(1-x) \sum_{n=0}^{\infty} x^{n}=1$;

3. $\left\|\sum_{n=0}^{\infty} x^{n}\right\| \leq 1$.

Demonstração: As afirmações são claramente verificadas para $x=0$. Então suponhamos que $x \neq 0$.

Primeiramente, provaremos que $\sum_{n=0}^{\infty} a_{n} x^{n}$ converge e, portanto, em particular, obtemos que $\sum_{n=0}^{\infty} x^{n}$ converge. Observemos que, pela Proposição 1.4.2, é suficiente provarmos que $\lim _{n \rightarrow \infty}\left\|a_{n} x^{n}\right\|=0$. 
Pela Proposição 1.3.7 (2) e (4), temos que

$$
0 \leq\left\|a_{n} x^{n}\right\|=\left\|x^{n}\right\| \leq\|x\|^{n}, \forall n \in \mathbb{N} .
$$

Como $\|x\|<1$, segue que $\lim _{n \rightarrow \infty}\|x\|^{n}=0$ e, portanto, $\lim _{n \rightarrow \infty}\left\|a_{n} x^{n}\right\|=0$.

A seguir, provaremos a afirmação 2 ..

Seja $y:=\sum_{n=0}^{\infty} x^{n}$ e, para cada $n \in \mathbb{N}$, consideremos $l_{n}:=\sum_{k=0}^{n} x^{k}$. Então $\lim _{n \rightarrow \infty} l_{n}=y$ e, conseqüentemente, $\lim _{n \rightarrow \infty}\left((1-x) l_{n}\right)=(1-x) y$ (pois, $\bar{K}$ é um anel topológico).

Notemos que

$$
(1-x) l_{n}=(1-x) \sum_{k=0}^{n} x^{k}=\sum_{k=0}^{n} x^{k}-\sum_{k=0}^{n} x^{k+1}=x^{0}-x^{n+1}=1-x^{n+1}
$$

e, como $\lim _{n \rightarrow \infty} x^{n}=0$, pois $\sum_{n=0}^{\infty} x^{n}$ converge, segue que $\lim _{n \rightarrow \infty}\left((1-x) l_{n}\right)=\lim _{n \rightarrow \infty}\left(1-x^{n+1}\right)=1$. Portanto, temos que $(1-x) y=1$, ou seja, 2. está satisfeita.

Finalmente, para a afirmação 3 , observemos que, de $\lim _{n \rightarrow \infty} l_{n}=y$, existe $n_{0} \in \mathbb{N}$ tal que $\left\|l_{n_{0}}-y\right\|<1$. Logo, pela Proposição $1.3 .7(1),(2)$ e (4), temos que

$$
\|y\| \leq \max \left\{\left\|y-l_{n_{0}}\right\|,\left\|l_{n_{0}}\right\|\right\} \leq \max \left\{1, \max \left\{\|x\|^{j}: 0 \leq j \leq n_{0}\right\}\right\} \leq 1 .
$$

Proposição 1.4.7 As seguintes asserções são verdadeiras:

1. se $a \in \operatorname{Inv}(\bar{K})$, então $\|a\| \neq 0$ e $\left\|a^{-1}\right\| \neq 0$;

2. se $a \in \operatorname{Inv}(\overline{\mathbb{K}})$ e $r:=\left\|a^{-1}\right\|^{-1}$, então $B_{r}(a) \subset \operatorname{Inv}(\overline{\mathbb{K}})$;

3. se $a \in \operatorname{Inv}(\overline{\mathbb{K}})$ e $r:=\left\|a^{-1}\right\|^{-1}$, então $\left\|z^{-1}\right\| \leq \frac{1}{r}$, para todo $z \in B_{r}(a)$;

4. $\operatorname{Inv}(\overline{\mathbb{K}})$ é um subconjunto aberto de $\overline{\mathbb{I}}$.

Demonstração: Para 1. basta notarmos que $1=\|1\|=\left\|a a^{-1}\right\| \leq\|a\|\left\|a^{-1}\right\|$ (Proposição 1.3.7 (2) e (6)). A afirmação 4. segue de 2.. Provaremos, a seguir, as afirmações 2. e 3 .. 
Sejam $a \in \operatorname{Inv}(\overline{\mathbb{K}})$ e $r:=\left\|a^{-1}\right\|^{-1}$. Fixemos $z \in B_{r}(a)$ e consideremos $x:=a^{-1}(a-z)$. Pela Proposição 1.3.7 (2), temos que

$$
\|x\|=\left\|a^{-1}(a-z)\right\| \leq\left\|a^{-1}\right\|\|a-z\|<\left\|a^{-1}\right\|\left\|a^{-1}\right\|^{-1}=1 .
$$

Então, pela Proposição $1.4 .6(2)$ e $(3)$, obtemos que $(1-x) \sum_{n=0}^{\infty} x^{n}=1$ e $\left\|\sum_{n=0}^{\infty} x^{n}\right\| \leq 1$.

Notemos que

$$
1-x=1-a^{-1}(a-z)=1-1+a^{-1} z=a^{-1} z=z a^{-1} .
$$

Logo, $1=z a^{-1} \sum_{n=0}^{\infty} x^{n}=z\left(a^{-1} \sum_{n=0}^{\infty} x^{n}\right)$, o que implica que $z \in \operatorname{Inv}(\overline{\mathbb{K}}) \mathrm{e}$

$$
\left\|z^{-1}\right\|=\left\|a^{-1} \sum_{n=0}^{\infty} x^{n}\right\| \leq\left\|a^{-1}\right\|\left\|\sum_{n=0}^{\infty} x^{n}\right\| \leq \frac{1}{r}
$$

onde a primeira desigualdade decorre da Proposição 1.3 .7 (2). Logo, as afirmações 2. e 3. são verdadeiras.

Notamos que $\operatorname{Inv}(\overline{\mathbb{K}})$ é um subconjunto denso de $\bar{K}$. Esse resultado foi provado por J. Aragona e S. O. Juriaans ([AJ]).

Na Proposição 1.4.6 estudamos a convergência da série $\sum_{n=0}^{\infty} a_{n} x^{n}$ no caso em que $\left(a_{n}\right)_{n \in N}$ é uma seqüência de elementos de $\mathbb{K}$ (resultado extraído de [AJ]). No próximo resultado, apresentaremos algumas condições para termos $\sum_{n=0}^{\infty} a_{n} x^{n}$ convergente, no caso em que $\left(a_{n}\right)_{n \in N}$ é uma seqüência de elementos de $\overline{I K}$. Esse resultado, além de interessante, será útil no capítulo 4.

Proposição 1.4.8 Seja $\left(a_{n}\right)_{n \in \mathbb{N}}$ uma seqüência de elementos de $\overline{\mathbb{K}}$. Se existem $\eta \in \mathbb{I}$, $k \in \mathbb{R}$ e $\left(p_{n}\right)_{n \in N}$ uma seqüência de elementos de $\mathbb{R}$ tais que

$$
\lim _{n \rightarrow \infty}\left(p_{n}+k n\right)=+\infty
$$

e, para cada $n \in \mathbb{N}$, existe $\left(a_{1 n \varepsilon}\right)_{\varepsilon}$ representante de $a_{n}$ tal que 


$$
\left|a_{1 n \varepsilon}\right| \leq \varepsilon^{p_{n}}, \forall \varepsilon \in \mathbf{I}_{\eta} \quad \text { e } \forall n \in \mathbb{N},
$$

então, para todo $x \in \overline{\mathbb{K}}$ com $\|x\|<e^{-|k|}$, tem-se que:

1. $\sum_{n=0}^{\infty} a_{n} x^{n}$ é convergente em $\overline{\mathbb{K}}$;

2. existe $\left(x_{\varepsilon}\right)_{\varepsilon}$ representante de $x$ e, para cada $n \in \mathbb{N}$, existe $\left(a_{n \varepsilon}\right)_{\varepsilon}$ representante de $a_{n}$ tais que $\sum_{n=0}^{\infty} a_{n \varepsilon} x_{\varepsilon}^{n}$ é convergente em $\mathbb{K}$, para todo $\varepsilon \in \mathbb{I},\left(\sum_{n=0}^{\infty} a_{n \varepsilon} x_{\varepsilon}^{n}\right)_{\varepsilon} \in \mathcal{E}_{M}(\mathbb{K}) e$

$$
\sum_{n=0}^{\infty} a_{n} x^{n}=\left[\left(\sum_{n=0}^{\infty} a_{n \varepsilon} x_{\varepsilon}^{n}\right)_{\varepsilon}\right]
$$

Demonstração: Notemos que $\left|a_{n}\right| \leq \alpha_{p_{n}}$, para todo $n \in \mathbb{N}$. Logo, pela Proposição 1.3.7 $(2),(8)$ e $(10)$, temos que

$$
\left\|a_{n} x^{n}\right\| \leq\left\|a_{n}\right\|\left\|x^{n}\right\| \leq\left\|a_{n}\right\|\|x\|^{n} \leq\left\|\alpha_{p_{n}}\right\| e^{-|k| n}=e^{-p_{n}-|k| n}
$$

para todo $n \in \mathbb{N}$ e, como $\lim _{n \rightarrow \infty}\left(p_{n}+k n\right)=+\infty$, segue que $\lim _{n \rightarrow \infty}\left(p_{n}+|k| n\right)=+\infty$ e, portanto, $\lim _{n \rightarrow \infty}\left\|a_{n} x^{n}\right\|=0$. Logo, pela Proposição 1.4.2, obtemos que $\sum_{n=0}^{\infty} a_{n} x^{n}$ é convergente em $\overline{\mathbb{K}}$, o que prova 1 ..

A seguir, provaremos a afirmação $2 .$. Seja $\left(x_{1 \varepsilon}\right)_{\varepsilon}$ um representante de $x$.

Observemos que $\mathcal{V}(x)=+\infty$ ou $|k|<\mathcal{V}(x)<+\infty$, pois $\|x\|<e^{-|k|}$, o que implica, pela Proposição 1.3.4 (3), que $|k| \in A(x)$ e deste último fato obtemos $x \alpha_{-|k|} \approx 0$. Então existe $\eta_{1} \in \mathbf{I}$ tal que $\frac{\left|x_{1 \varepsilon}\right|}{\varepsilon^{|k|}} \leq \frac{1}{2}$, para todo $\varepsilon \in \mathbf{I}_{\eta_{1}}$. Seja $\eta_{2}:=\min \left\{\eta, \eta_{1}\right\}$ e consideremos, para cada $\varepsilon \in \mathbf{I}$,

$$
x_{\varepsilon}:=\left\{\begin{array}{ll}
x_{1 \varepsilon}, & \text { se } 0<\varepsilon<\eta_{2} \\
0 & , \text { se } \eta_{2} \leq \varepsilon \leq 1
\end{array} \quad \text { e } \quad a_{n \varepsilon}:=\left\{\begin{array}{ll}
a_{1 n \varepsilon}, & \text { se } 0<\varepsilon<\eta_{2} \\
0 \quad, & \text { se } \eta_{2} \leq \varepsilon \leq 1
\end{array}, \forall n \in \mathbb{N}\right.\right.
$$

Logo, $\left(x_{\varepsilon}\right)_{\varepsilon}$ é um representante de $x$ e, para cada $n \in \mathbb{N},\left(a_{n \varepsilon}\right)_{\varepsilon}$ é um representante de $a_{n}$ tais que $\frac{\left|x_{\varepsilon}\right|}{\varepsilon^{|k|}} \leq \frac{1}{2}$ e $\left|a_{n \varepsilon}\right| \leq \varepsilon^{p_{n}}$, para todo $\varepsilon \in \mathbf{I}$. Assim, temos que

$$
\left|a_{n \varepsilon} x_{\varepsilon}^{n}\right|=\left|a_{n \varepsilon}\right|\left|x_{\varepsilon}\right|^{n} \leq \varepsilon^{p_{n}} \frac{\varepsilon^{|k| n}}{2^{n}}, \quad \forall \varepsilon \in \mathbf{I}, \quad \forall n \in \mathbb{N} .
$$


Notemos que, como $\lim _{n \rightarrow \infty}\left(p_{n}+k n\right)=+\infty$, existe $n_{0} \in \mathbb{N}$ tal que $p_{n}+k n>1$, para todo $n \geq n_{0}$. Desse modo, $\varepsilon^{p_{n}}<\varepsilon^{1-k n}$, para todo $n \geq n_{0}$, e então, de (1.4), temos

$$
\left|a_{n \varepsilon} x_{\varepsilon}^{n}\right|<\varepsilon^{1-k n} \frac{\varepsilon^{|k| n}}{2^{n}}=\frac{\left(\varepsilon^{|k|-k}\right)^{n} \varepsilon}{2^{n}} \leq \frac{1}{2^{n}}, \quad \forall \varepsilon \in \mathbf{I}, \quad \forall n \geq n_{0},
$$

e, portanto, como $\sum_{n=0}^{\infty} 2^{-n}$ converge, segue que $\sum_{n=0}^{\infty} a_{n \varepsilon} x_{\varepsilon}^{n}$ converge, para todo $\varepsilon \in \mathbf{I}$. Além disso, temos que $\left|\sum_{n=0}^{\infty} a_{n \varepsilon} x_{\varepsilon}^{n}\right| \leq \sum_{n=0}^{\infty} 2^{-n}=2$, para todo $\varepsilon \in \mathbf{I}$, o que implica que $\left(\sum_{n=0}^{\infty} a_{n \varepsilon} x_{\varepsilon}^{n}\right)_{\varepsilon}$ $\in \mathcal{E}_{M}(\mathbb{K}) \mathrm{e}$, assim, podemos definir $c:=\left[\left(\sum_{n=0}^{\infty} a_{n \varepsilon} x_{\varepsilon}^{n}\right)_{\varepsilon}\right] \in \bar{K}$.

Provaremos que $\sum_{n=0}^{\infty} a_{n} x^{n}=c$. Antes, porém, notemos que, se $l_{n}:=\sum_{j=0}^{n} a_{j} x^{j}$, para todo $n \in \mathbb{N}$, então

$$
\begin{aligned}
c-l_{n}=c-\sum_{j=0}^{n} a_{j} x^{j} & =\left[\left(\sum_{j=0}^{\infty} a_{j \varepsilon} x_{\varepsilon}^{j}\right)_{\varepsilon}\right]-\sum_{j=0}^{n}\left[\left(a_{j \varepsilon}\right)_{\varepsilon}\right]\left[\left(x_{\varepsilon}\right)_{\varepsilon}\right]^{j} \\
& =\left[\left(\sum_{j=0}^{\infty} a_{j \varepsilon} x_{\varepsilon}^{j}\right)_{\varepsilon}\right]-\left[\left(\sum_{j=0}^{n} a_{j \varepsilon} x_{\varepsilon}^{j}\right)_{\varepsilon}\right] \\
& =\left[\left(\sum_{j=n+1}^{\infty} a_{j \varepsilon} x_{\varepsilon}^{j}\right)_{\varepsilon}\right] .
\end{aligned}
$$

Seja $\delta>0$ e consideremos $\sigma:=\min \left\{\frac{1}{2}, \delta\right\}, d:=-\ln \sigma>0$ e $n_{1} \in \mathbb{N}$ tal que $p_{n}+k n>d+1$, para todo $n \geq n_{1}$. Então, por (1.4), para todo $\varepsilon \in \mathbf{I}$ e todo $n \geq n_{1}$, temos que

$$
\begin{aligned}
\frac{1}{\varepsilon^{d}}\left|\sum_{j=n+1}^{\infty} a_{j \varepsilon} x_{\varepsilon}^{j}\right| \leq \frac{1}{\varepsilon^{d}} \sum_{j=n+1}^{\infty}\left|a_{j \varepsilon} x_{\varepsilon}^{j}\right| \leq \sum_{j=n+1}^{\infty} \frac{\varepsilon^{p_{j}}}{\varepsilon^{d}} \frac{\varepsilon^{|k| j}}{2^{j}} & \leq \sum_{j=n+1}^{\infty} \frac{\varepsilon^{d+1-k j}}{\varepsilon^{d}} \frac{\varepsilon^{|k| j}}{2^{j}} \\
& =\varepsilon \sum_{j=n+1}^{\infty}\left(\varepsilon^{|k|-k}\right)^{j} \frac{1}{2^{j}} \\
& \leq \varepsilon \sum_{j=n+1}^{\infty} \frac{1}{2^{j}} \\
& \leq 2 \varepsilon .
\end{aligned}
$$

Logo,

$$
\lim _{\varepsilon \downarrow 0}\left(\frac{1}{\varepsilon^{d}}\left|\sum_{j=n+1}^{\infty} a_{j \varepsilon} x_{\varepsilon}^{j}\right|\right)=0, \quad \forall n \geq n_{1}
$$


ou seja,

$$
\left(c-l_{n}\right) \alpha_{-d} \approx 0, \forall n \geq n_{1}
$$

Portanto,

$$
\left\|c-l_{n}\right\|=\left\|l_{n}-c\right\| \leq e^{-d}=\sigma<\delta, \quad \forall n \geq n_{1} .
$$

Assim,

$$
\sum_{n=0}^{\infty} a_{n} x^{n}=\lim _{n \rightarrow \infty} l_{n}=c=\left[\left(\sum_{n=0}^{\infty} a_{n \varepsilon} x_{\varepsilon}^{n}\right)_{\varepsilon}\right]
$$

No que segue, usaremos a seguinte notação

$$
\overline{\mathbb{R}}_{+}:=\{x \in \overline{\mathbb{R}}: x \geq 0\} .
$$

A seguir, veremos que, como no contexto clássico, pode-se determinar a raiz quadrada de um número real generalizado q-positivo e pode-se definir um produto escalar generalizado em $\overline{\mathbb{K}}^{n}$ para o qual vale uma desigualdade de Cauchy-Schwarz generalizada.

Proposição 1.4.9 Para cada $x \in \overline{\mathbb{R}}_{+}$, existe um único $y \in \overline{\mathbb{R}}_{+}$tal que $y^{2}=x$.

Demonstração: Seja $\left(x_{\varepsilon}\right)_{\varepsilon}$ um representante de $x$ tal que $x_{\varepsilon} \geq 0$, para todo $\varepsilon \in \mathbf{I}$. Consideremos, para cada $\varepsilon \in \mathbf{I}, y_{\varepsilon}:=\sqrt{x_{\varepsilon}}$. Claramente, temos que $\left(y_{\varepsilon}\right)_{\varepsilon} \in \mathcal{E}_{M}(\mathbb{R})$. Logo, $y:=\left[\left(y_{\varepsilon}\right)_{\varepsilon}\right] \in \overline{\mathbb{R}}$ é tal que $y^{2}=x$ e $y \geq 0$.

A seguir, provaremos a unicidade.

Sejam $z \in \mathbb{R}_{+}$tal que $z^{2}=x$ e $\left(z_{\varepsilon}\right)_{\varepsilon}$ um representante de $z$ tal que $z_{\varepsilon} \geq 0$, para todo $\varepsilon \in$ I. Então

$$
\left|y_{\varepsilon}-z_{\varepsilon}\right| \leq\left|y_{\varepsilon}\right|+\left|z_{\varepsilon}\right|=y_{\varepsilon}+z_{\varepsilon}, \forall \varepsilon \in \mathbf{I} \text {, }
$$

o que implica que

$\left|y_{\varepsilon}-z_{\varepsilon}\right|^{2}=\left|y_{\varepsilon}-z_{\varepsilon}\right|\left|y_{\varepsilon}-z_{\varepsilon}\right| \leq\left(y_{\varepsilon}+z_{\varepsilon}\right)\left|y_{\varepsilon}-z_{\varepsilon}\right|=\left|\left(y_{\varepsilon}+z_{\varepsilon}\right)\left(y_{\varepsilon}-z_{\varepsilon}\right)\right|=\left|y_{\varepsilon}^{2}-z_{\varepsilon}^{2}\right|, \forall \varepsilon \in \mathbf{I}$. 
Como $y^{2}=z^{2}$, temos que $\left(y_{\varepsilon}^{2}-z_{\varepsilon}^{2}\right)_{\varepsilon} \in \mathcal{N}(\mathbb{R})$. Logo, $\lim _{\varepsilon \downarrow 0} \frac{\left|y_{\varepsilon}^{2}-z_{\varepsilon}^{2}\right|}{\varepsilon^{2 b}}=0$, para todo $b \in \mathbb{R}$, e assim obtemos que

$$
\lim _{\varepsilon \downarrow 0} \frac{\left|y_{\varepsilon}-z_{\varepsilon}\right|^{2}}{\varepsilon^{2 b}}=0, \forall b \in \mathbb{R}
$$

Portanto

$$
\lim _{\varepsilon \downarrow 0} \frac{\left|y_{\varepsilon}-z_{\varepsilon}\right|}{\varepsilon^{b}}=0, \forall b \in \mathbb{R}
$$

ou seja, $z=y$.

Em virtude do resultado anterior faz sentido a definição a seguir.

Definição 1.4.10 Para cada $x \in \mathbb{R}_{+}$, chama-se de raiz quadrada q-positiva de $x$, e denota-se por $\sqrt{x}$ ou por $x^{\frac{1}{2}}$, o único $y \in \overline{\mathbb{R}}_{+}$tal que $y^{2}=x$.

Definição 1.4.11 Define-se $[\cdot]_{2}: \bar{K}^{n} \longrightarrow \overline{\mathbb{R}}$ por

$$
\left[\left(x_{1}, \ldots, x_{n}\right)\right]_{2}:=\left(\sum_{j=1}^{n}\left|x_{j}\right|^{2}\right)^{\frac{1}{2}}
$$

onde $|\cdot|$ é como na Definição 1.1.13.

Notamos que, se $x=\left(x_{1}, \ldots, x_{n}\right) \in \overline{\mathbb{K}}^{n}$ e $\left(x_{j \varepsilon}\right)_{\varepsilon}$ é um representante de $x_{j}$, para $1 \leq j \leq n$, então

$$
[x]_{2}=\left[\left(\left(\sum_{j=1}^{n}\left|x_{j \varepsilon}\right|^{2}\right)^{\frac{1}{2}}\right)_{\varepsilon}\right]
$$

pois $\left[\left(\left(\sum_{j=1}^{n}\left|x_{j \varepsilon}\right|^{2}\right)^{\frac{1}{2}}\right)_{\varepsilon}\right] \geq 0 \quad$ e $\left[\left(\left(\sum_{j=1}^{n}\left|x_{j \varepsilon}\right|^{2}\right)^{\frac{1}{2}}\right)_{\varepsilon}\right]^{2}=\sum_{j=1}^{n}\left|x_{j}\right|^{2}$.

Em $\overline{\mathbb{K}}^{n}$ definiremos um produto escalar generalizado. Para essa finalidade, notamos que, se $x \in \bar{K}$ e $\left(x_{\varepsilon}\right)_{\varepsilon}$ e $\left(x_{1 \varepsilon}\right)_{\varepsilon}$ são representantes de $x$, então $\left(\overline{x_{\varepsilon}}-\overline{x_{1 \varepsilon}}\right)_{\varepsilon} \in \mathcal{N}(\mathbb{K})$, onde $\overline{x_{\varepsilon}}$ 
e $\overline{x_{1 \varepsilon}}$ são os conjugados de $x_{\varepsilon}$ e $x_{1 \varepsilon}$, respectivamente, para todo $\varepsilon \in \mathbf{I}$. Logo, faz sentido a seguinte definição:

Definição 1.4.12 Para cada $x \in \bar{K}$, chama-se de conjugado de $x$, e denota-se por $\bar{x}$, o elemento de $\bar{K}$ dado por $\bar{x}:=\left[\left(\overline{x_{\varepsilon}}\right)_{\varepsilon}\right]$, onde $\left(x_{\varepsilon}\right)_{\varepsilon}$ é um representante qualquer de $x$.

É fácil verificar que, se $x, y \in \overline{\mathbb{K}}$, então $\overline{x+y}=\bar{x}+\bar{y}, \quad \overline{x y}=\bar{x} \bar{y} \quad$ e $\quad \overline{\bar{x}}=x$.

Definição 1.4.13 Define-se $\langle\cdot, \cdot\rangle: \overline{\mathbb{K}}^{n} \times \overline{\mathbb{K}}^{n} \longrightarrow \overline{\mathbb{K}}$ por

$$
\left\langle\left(x_{1}, \ldots, x_{n}\right),\left(y_{1}, \ldots, y_{n}\right)\right\rangle:=\sum_{j=1}^{n} x_{j} \overline{y_{j}} .
$$

Proposição 1.4.14 Sejam $x, y, z \in \overline{\mathbb{K}}^{n}$ e $\lambda \in \bar{K}$. Tem-se que

1. $\langle x+y, z\rangle=\langle x, z\rangle+\langle y, z\rangle$;

2. $\langle\lambda x, y\rangle=\lambda\langle x, y\rangle$;

3. $\overline{\langle y, x\rangle}=\langle x, y\rangle$;

4. $\langle\cdot, \cdot\rangle$ é uma aplicação $\overline{\mathbb{K}}$-sesquilinear;

5. se $\langle x, x\rangle=0$, então $x=0$;

6. $[x]_{2}=\sqrt{|\langle x, x\rangle|}$.

Demonstração: Denotemos por $x_{j}, y_{j}$ e $z_{j}$ as j-ésimas coordenadas de $x, y$ e $z$, respectivamente. Temos que

$$
\begin{aligned}
\langle x+y, z\rangle & :=\sum_{j=1}^{n}\left(x_{j}+y_{j}\right) \overline{z_{j}}=\left(\sum_{j=1}^{n} x_{j} \overline{z_{j}}\right)+\left(\sum_{j=1}^{n} y_{j} \overline{z_{j}}\right)=\langle x, z\rangle+\langle y, z\rangle ; \\
\langle\lambda x, y\rangle & :=\sum_{j=1}^{n} \lambda x_{j} \overline{y_{j}}=\lambda \sum_{j=1}^{n} x_{j} \overline{y_{j}}=\lambda\langle x, y\rangle
\end{aligned}
$$




$$
\overline{\langle y, x\rangle}:=\overline{\sum_{j=1}^{n} y_{j} \overline{x_{j}}}=\sum_{j=1}^{n} \overline{y_{j}} x_{j}=\sum_{j=1}^{n} x_{j} \overline{y_{j}}=\langle x, y\rangle ;
$$

o que prova $1 ., 2$. e $3 .$.

Notemos que a afirmação 4 . é conseqüência das anteriores.

Seja $\left(x_{j \varepsilon}\right)_{\varepsilon}$ um representante de $x_{j}$, para cada $1 \leq j \leq n$. Observemos que

$$
\langle x, x\rangle=\sum_{j=1}^{n} x_{j} \overline{x_{j}}=\left(\sum_{j=1}^{n} x_{j \varepsilon} \overline{x_{j \varepsilon}}\right)_{\varepsilon}+\mathcal{N}(\mathbb{K})
$$

e assim

$$
|\langle x, x\rangle|=\left(\left|\sum_{j=1}^{n} x_{j \varepsilon} \overline{x_{j \varepsilon}}\right|\right)_{\varepsilon}+\mathcal{N}(\mathbb{R})=\left(\sum_{j=1}^{n}\left|x_{j \varepsilon}\right|^{2}\right)_{\varepsilon}+\mathcal{N}(\mathbb{R})=\sum_{j=1}^{n}\left|x_{j}\right|^{2},
$$

o que prova 6.

Suponhamos que $\langle x, x\rangle=0$. Então, por (1.5), temos que $\left(\sum_{j=1}^{n} x_{j \varepsilon} \overline{x_{j \varepsilon}}\right)_{\varepsilon} \in \mathcal{N}(\mathbb{K}) \mathrm{e}$ portanto, dado $q \in \mathbb{N}$ existem $c>0$ e $\eta \in \mathbf{I}$ tais que

$$
\sum_{j=1}^{n}\left|x_{j \varepsilon}\right|^{2}=\left|\sum_{j=1}^{n} x_{j \varepsilon} \overline{x_{j \varepsilon}}\right| \leq c \varepsilon^{2 q}, \forall \varepsilon \in \mathbf{I}_{\eta}
$$

Logo, para cada $1 \leq j \leq n$, temos que

$$
\left|x_{j \varepsilon}\right| \leq c^{\frac{1}{2}} \varepsilon^{q}, \forall \varepsilon \in \mathbf{I}_{\eta}
$$

e, pela Proposição 1.1 .5 , obtemos $x_{j}=0$, para todo $1 \leq j \leq n$. Portanto, $x=0$, o que prova 5 ..

Lema 1.4.15 (Desigualdade de Cauchy-Schwarz generalizada) Sejam $x, y \in \overline{\mathbb{K}}^{n}$. Então $|\langle x, y\rangle| \leq[x]_{2}[y]_{2}$.

Demonstração: Sejam $x=\left(x_{1}, \ldots, x_{n}\right), y=\left(y_{1}, \ldots, y_{n}\right) \in \bar{K}^{n}$ e $\left(x_{j \varepsilon}\right)_{\varepsilon}$ e $\left(y_{j \varepsilon}\right)_{\varepsilon}$ representantes de $x_{j}$ e $y_{j}$, respectivamente, para cada $1 \leq j \leq n$.

Notemos que

$$
|\langle x, y\rangle|=\left|\sum_{j=1}^{n} x_{j} \overline{y_{j}}\right|=\left|\left[\left(\sum_{j=1}^{n} x_{j \varepsilon} \overline{y_{j \varepsilon}}\right)_{\varepsilon}\right]\right|=\left[\left(\left|\sum_{j=1}^{n} x_{j \varepsilon} \overline{y_{j \varepsilon}}\right|\right)_{\varepsilon}\right],
$$


e, como

$$
\left|\sum_{j=1}^{n} x_{j \varepsilon} \overline{y_{j \varepsilon}}\right| \leq \sum_{j=1}^{n}\left|x_{j \varepsilon} \overline{y_{j \varepsilon}}\right|=\sum_{j=1}^{n}\left|x_{j \varepsilon}\right|\left|y_{j \varepsilon}\right| \leq\left(\sum_{j=1}^{n}\left|x_{j \varepsilon}\right|^{2}\right)^{\frac{1}{2}}\left(\sum_{j=1}^{n}\left|y_{j \varepsilon}\right|^{2}\right)^{\frac{1}{2}}
$$

para todo $\varepsilon \in \mathbf{I}$, e

$$
[x]_{2}[y]_{2}=\left[\left(\left(\sum_{j=1}^{n}\left|x_{j \varepsilon}\right|^{2}\right)^{\frac{1}{2}}\right)_{\varepsilon}\right]\left[\left(\left(\sum_{j=1}^{n}\left|y_{j \varepsilon}\right|^{2}\right)^{\frac{1}{2}}\right)_{\varepsilon}\right],
$$

concluímos que $|\langle x, y\rangle| \leq[x]_{2}[y]_{2}$.

$\mathrm{Na}$ seção a seguir, apresentaremos as funções generalizadas de Colombeau e algumas propriedades e definições relacionadas a elas que nos serão úteis.

\subsection{Definição e algumas propriedades de $\mathcal{G}(\Omega ; \mathbb{K})$}

Para facilitar a leitura do trabalho, apresentamos, nesta seção, a álgebra das funções generalizadas de Colombeau (versão simplificada) e algumas de suas propriedades. As provas dos resultados aqui apresentados, com exceção do Teorema 1.5.6, da Proposição 1.5.12 e do Exemplo 1.5.14, podem ser encontradas em [Sil-A].

No restante da Dissertação, se $\Omega$ é um subconjunto aberto de $\mathbb{R}^{n}$ e $F: \Omega \longrightarrow \mathbb{K}$ é uma função limitada em $X \subset \Omega$, então a notação $\|F(\cdot)\|_{X}$ denotará o supremo do conjunto $\{|F(x)|: x \in X\}$, isto é,

$$
\|F(\cdot)\|_{X}:=\sup _{x \in X}|F(x)|
$$

Definição 1.5.1 Seja $\Omega$ um subconjunto aberto de $\mathbb{R}^{n}$. Define-se $\mathcal{E}[\Omega ; \mathbb{K}]:=\left(\mathscr{C}^{\infty}(\Omega ; \mathbb{K})\right)^{\mathbf{I}}$.

Definição 1.5.2 Sejam $\Omega$ um subconjunto aberto de $\mathbb{R}^{n}$ e $\left(f_{\varepsilon}\right)_{\varepsilon} \in \mathcal{E}[\Omega ; \mathbb{K}]$. Diz-se que $\left(f_{\varepsilon}\right)_{\varepsilon}$ pertence a $\mathcal{E}_{M}[\Omega ; \mathbb{K}]$ se, e somente se,

$\forall K \subset \subset \Omega e \forall \alpha \in \mathbb{N}^{n}, \exists \sigma \in \mathbb{R}$ tal que $\left\|\partial^{\alpha} f_{\varepsilon}(\cdot)\right\|_{K}=o\left(\varepsilon^{\sigma}\right)$, quando $\varepsilon \downarrow 0$. 
Um elemento de $\mathcal{E}_{M}[\Omega ; \mathbb{K}]$ é chamado de função moderada em $\Omega$. Observamos que o conjunto $\mathcal{E}_{M}[\Omega ; \mathbb{K}]$ munido das operações usuais de soma e de produto de funções e de produto de um elemento de $\mathbb{K}$ por uma função é uma $\mathbb{K} K$-álgebra.

Definição 1.5.3 Seja $\Omega$ um subconjunto aberto de $\mathbb{R}^{n}$. Denota-se por $\mathcal{N}[\Omega ; \mathbb{K}]$ o conjunto das funções $\left(f_{\varepsilon}\right)_{\varepsilon} \in \mathcal{E}[\Omega ; \mathbb{K}]$ tais que

$\forall K \subset \subset \Omega, \forall \alpha \in \mathbb{N}^{n} e \forall b \in \mathbb{R}$ tem-se que $\left\|\partial^{\alpha} f_{\varepsilon}(\cdot)\right\|_{K}=o\left(\varepsilon^{b}\right)$, quando $\varepsilon \downarrow 0$.

Um elemento de $\mathcal{N}[\Omega ; \mathbb{K}]$ é chamado de função nula em $\Omega$. É fácil verificar que o conjunto $\mathcal{N}[\Omega ; \mathbb{K}]$ é um ideal de $\mathcal{E}_{M}[\Omega ; \mathbb{K}]$.

Definição 1.5.4 Seja $\Omega$ um subconjunto aberto de $\mathbb{R}^{n}$. Denota-se por $\mathcal{G}(\Omega ; \mathbb{K})$ a álgebra quociente de $\mathcal{E}_{M}[\Omega ; \mathbb{K}]$ por $\mathcal{N}[\Omega ; \mathbb{K}]$.

Um elemento de $\mathcal{G}(\Omega ; \mathbb{K})$ é chamado de função generalizada em $\Omega$. É fácil verificar que o anel quociente $\mathcal{G}(\Omega ; \mathbb{K})$ é uma $\mathbb{K}$-álgebra.

Se $\left(f_{\varepsilon}\right)_{\varepsilon} \in \mathcal{E}_{M}[\Omega ; \mathbb{K}]$, denotaremos por $\left[\left(f_{\varepsilon}\right)_{\varepsilon}\right]$ a classe de $\left(f_{\varepsilon}\right)_{\varepsilon}$ em $\mathcal{G}(\Omega ; \mathbb{K})$, isto é, $\left[\left(f_{\varepsilon}\right)_{\varepsilon}\right]=\left(f_{\varepsilon}\right)_{\varepsilon}+\mathcal{N}[\Omega ; \mathbb{K}]$, e escreveremos $f=\left[\left(f_{\varepsilon}\right)_{\varepsilon}\right]$.

No restante do trabalho, a menos de menção em contrário, denotaremos $\mathscr{C}^{\infty}(\Omega ; \mathbb{K})$, $\mathcal{E}[\Omega ; \mathbb{K}], \mathcal{E}_{M}[\Omega ; \mathbb{K}], \mathcal{N}[\Omega ; \mathbb{K}]$ e $\mathcal{G}(\Omega ; \mathbb{K})$, respectivamente, por $\mathscr{C}^{\infty}(\Omega), \mathcal{E}[\Omega], \mathcal{E}_{M}[\Omega], \mathcal{N}[\Omega]$ e $\mathcal{G}(\Omega)$.

Apresentaremos, a seguir, uma caracterização para funções moderadas e para funções nulas. Tal caracterização é usada em vários trabalhos para definir a álgebra $\mathcal{G}(\Omega)$ e será utilizada, ao longo deste trabalho, sempre que for conveniente. 
Proposição 1.5.5 Sejam $\Omega$ um subconjunto aberto de $\mathbb{R}^{n}$ e $\left(f_{\varepsilon}\right)_{\varepsilon} \in \mathcal{E}[\Omega]$.

1. São equivalentes:

(a) $\left(f_{\varepsilon}\right)_{\varepsilon} \in \mathcal{E}_{M}[\Omega]$;

(b) para todo $K \subset \subset \Omega$ e para todo $\alpha \in \mathbb{N}^{n}$, existem $N \in \mathbb{N}, c>0$ e $\eta \in \mathbf{I}$ tais que

$$
\left\|\partial^{\alpha} f_{\varepsilon}(\cdot)\right\|_{K} \leq c \varepsilon^{-N}, \forall \varepsilon \in \mathbf{I}_{\eta}
$$

2. São equivalentes:

(a) $\left(f_{\varepsilon}\right)_{\varepsilon} \in \mathcal{N}[\Omega]$;

(b) para todo $K \subset \subset \Omega$, todo $\alpha \in \mathbb{N}^{n}$ e todo $q \in \mathbb{N}$, existem $c>0$ e $\eta \in \mathbf{I}$ tais que

$$
\left\|\partial^{\alpha} f_{\varepsilon}(\cdot)\right\|_{K} \leq c \varepsilon^{q}, \forall \varepsilon \in \mathbf{I}_{\eta}
$$

Demonstração: Ver [Sil-A, Proposição 1.1.4 e Proposição 1.1.5].

O Teorema a seguir o qual pode ser encontrado em [GKOS], nos mostra que para uma função $\left(f_{\varepsilon}\right)_{\varepsilon} \in \mathcal{E}_{M}[\Omega]$ ser nula é suficiente verificarmos a condição 2.(b) da Proposição 1.5.5 para $\alpha=0$.

Teorema 1.5.6 Sejam $\Omega$ um subconjunto aberto de $\mathbb{R}^{n}$ e $\left(f_{\varepsilon}\right)_{\varepsilon} \in \mathcal{E}_{M}[\Omega]$. Então $\left(f_{\varepsilon}\right)_{\varepsilon} \in$ $\mathcal{N}[\Omega]$ se, e somente se,

$$
\forall K \subset \subset \Omega e \forall m \in \mathbb{N}, \exists c>0 e \exists \eta \in \mathbf{I} \text { tais que }\left\|f_{\varepsilon}(\cdot)\right\|_{K} \leq c \varepsilon^{m}, \forall \varepsilon \in \mathbf{I}_{\eta} .
$$

Demonstração: Se $\left(f_{\varepsilon}\right)_{\varepsilon} \in \mathcal{N}[\Omega]$, então, pela Proposição 1.5.5, segue diretamente o resultado.

Suponhamos, então, que $\left(f_{\varepsilon}\right)_{\varepsilon}$ satisfaça (1.6). Podemos supor que $\mathbb{K}=\mathbb{R}$ pois, caso contrário, o cálculo que se segue pode ser feito, separadamente, para a parte real e imaginária de $f_{\varepsilon}$. 
Provaremos, por indução sobre $|\alpha|$, onde $\alpha \in \mathbb{N}^{n}$, que

$\forall K \subset \subset \Omega$ e $\forall m \in \mathbb{N}, \exists c>0$ e $\exists \eta \in \mathbf{I}$ tais que $\left\|\partial f_{\varepsilon}^{\alpha}(\cdot)\right\|_{K} \leq c \varepsilon^{m}, \forall \varepsilon \in \mathbf{I}_{\eta}$.

É claro, por (1.6), que (1.7) é verdadeira se $|\alpha|=0$. Seja $\alpha=\left(\alpha_{1}, \ldots, \alpha_{n}\right) \in \mathbb{N}^{n} \mathrm{com}$ $|\alpha|>0$ e suponha que (1.7) seja verdadeira para todo $\gamma \in \mathbb{N}^{n} \operatorname{com}|\gamma|=|\alpha|-1$. Como $|\alpha|>0$, existe $1 \leq i \leq n$ tal que $\alpha_{i} \neq 0$. Observemos que

$$
\partial^{\alpha} f_{\varepsilon}=\frac{\partial^{|\alpha|} f_{\varepsilon}}{\partial x_{1}^{\alpha_{1}} \ldots \partial x_{i}^{\alpha_{i}} \ldots \partial x_{n}^{\alpha_{n}}}=\frac{\partial}{\partial x_{i}}\left(\frac{\partial^{|\alpha|-1} f_{\varepsilon}}{\partial x_{1}^{\alpha_{1}} \ldots \partial x_{i}^{\alpha_{i}-1} \ldots \partial x_{n}^{\alpha_{n}}}\right)=\frac{\partial g_{\varepsilon}}{\partial x_{i}}
$$

onde $g_{\varepsilon}:=\frac{\partial^{|\alpha|-1} f_{\varepsilon}}{\partial x_{1}^{\alpha_{1}} \ldots \partial x_{i}^{\alpha_{i}-1} \ldots \partial x_{n}^{\alpha_{n}}} \in \mathcal{E}_{M}[\Omega]$, para todo $\varepsilon \in \mathbf{I}$.

Sejam $K \subset \subset \Omega$ e $m \in \mathbb{N}$. Consideremos $U$ um aberto de $\Omega$ tal que $K \subset U \subset \bar{U} \subset \subset \Omega$ e sejam $\delta:=\min \left\{1, d\left(K, U^{c}\right)\right\}>0$ e $L:=K+B_{\frac{\delta}{2}}^{\prime}(0) \subset \subset \mathbb{R}^{n}$. Então $\bigcup_{x \in K} B_{\frac{\delta}{2}}^{\prime}(x) \subset \Omega$ e, assim, como $y+w \in B_{\frac{\delta}{2}}^{\prime}(y)$, para todo $y \in K$ e todo $w \in B_{\frac{\delta}{2}}^{\prime}(0)$, temos que $K \subset L \subset \subset \Omega$. Denotemos $\frac{\partial}{\partial x_{i}}$ e $\frac{\partial^{2}}{\partial x_{i}^{2}}$ por $\partial_{i}$ e $\partial_{i}^{2}$, respectivamente. Pela Proposição 1.5.5 (1), para $\beta=\left(\beta_{1}, \ldots, \beta_{n}\right) \in \mathbb{N}^{n} \operatorname{com} \beta_{i}=2=|\beta|$, existem $N \in \mathbb{N}, c_{1}>0$ e $\eta_{1} \in \mathbf{I}$ tais que

$$
\left\|\partial_{i}^{2} g_{\varepsilon}(\cdot)\right\|_{L} \leq c_{1} \varepsilon^{-N}, \forall \varepsilon \in \mathbf{I}_{\eta_{1}}
$$

e, pela Hipótese de Indução, existem $c_{2}>0$ e $\eta_{2} \in \mathbf{I}$ tais que

$$
\left\|g_{\varepsilon}(\cdot)\right\|_{L} \leq c_{2} \varepsilon^{2 m+N+1}, \forall \varepsilon \in \mathbf{I}_{\eta_{2}}
$$

Sejam $x \in K, 0 \leq \varepsilon \leq \frac{\delta}{2} \leq 1$ e $\left\{e_{j}: 1 \leq j \leq n\right\}$ a base canônica de $\mathbb{R}^{n}$. Notemos que $t \varepsilon^{m+N+1} e_{i} \in B_{\frac{\delta}{2}}^{\prime}(0)$, para todo $0 \leq t \leq 1$, e, assim, o segmento de extremidades $x$ e $x+\varepsilon^{m+N+1} e_{i}$ está contido em $L \subset \Omega$. Observemos também que $f_{\varepsilon}$ é diferenciável, para todo $\varepsilon \in \mathbf{I}$, pois $\left(f_{\varepsilon}\right)_{\varepsilon} \in\left(\mathscr{C}^{\infty}(\Omega ; \mathbb{R})\right)^{\mathbf{I}}$. Desse modo, aplicando a Fórmula de Taylor, temos que

$$
g_{\varepsilon}\left(x+\varepsilon^{m+N+1} e_{i}\right)=g_{\varepsilon}(x)+\partial_{i} g_{\varepsilon}(x) \varepsilon^{m+N+1}+\frac{1}{2} \partial_{i}^{2} g_{\varepsilon}\left(x_{\theta}\right)\left(\varepsilon^{m+N+1}\right)^{2},
$$

onde, $x_{\theta}:=x+\theta \varepsilon^{m+N+1} e_{i}$, para algum $0<\theta<1$. Logo,

$$
\partial_{i} g_{\varepsilon}(x)=\frac{g_{\varepsilon}\left(x+\varepsilon^{m+N+1} e_{i}\right)}{\varepsilon^{m+N+1}}-\frac{g_{\varepsilon}(x)}{\varepsilon^{m+N+1}}-\frac{1}{2} \partial_{i}^{2} g_{\varepsilon}\left(x_{\theta}\right) \varepsilon^{m+N+1},
$$


e, portanto,

$$
\begin{gathered}
\left|\partial_{i} g_{\varepsilon}(x)\right| \leq \frac{\left|g_{\varepsilon}\left(x+\varepsilon^{m+N+1} e_{i}\right)\right|}{\varepsilon^{m+N+1}}+\frac{\left|g_{\varepsilon}(x)\right|}{\varepsilon^{m+N+1}}+\frac{1}{2}\left|\partial_{i}^{2} g_{\varepsilon}\left(x_{\theta}\right)\right| \varepsilon^{m+N+1} . \\
\text { Seja } \eta:=\min \left\{\eta_{1}, \eta_{2}, \frac{\delta}{2}\right\} \text {. Por (1.8) e }(1.9), \text { temos } \\
\left|\partial_{i}^{2} g_{\varepsilon}\left(x_{\theta}\right)\right| \varepsilon^{m+N+1} \leq c_{1} \varepsilon^{-N} \varepsilon^{m+N+1}=c_{1} \varepsilon^{m+1} \leq c_{1} \varepsilon^{m}, \quad \frac{\left|g_{\varepsilon}(x)\right|}{\varepsilon^{m+N+1}} \leq \frac{c_{2} \varepsilon^{2 m+N+1}}{\varepsilon^{m+N+1}}=c_{2} \varepsilon^{m}
\end{gathered}
$$

e

$$
\frac{\left|g_{\varepsilon}\left(x+\varepsilon^{m+N+1} e_{i}\right)\right|}{\varepsilon^{m+N+1}} \leq c_{2} \varepsilon^{2 m+N+1} \varepsilon^{-m-N-1}=c_{2} \varepsilon^{m},
$$

para todo $\varepsilon \in \mathbf{I}_{\eta}$. Logo, considerando $c:=2 c_{2}+\frac{1}{2} c_{1}$, obtemos

$$
\left|\partial^{\alpha} f_{\varepsilon}(x)\right|=\left|\partial_{i} g_{\varepsilon}(x)\right| \leq 2 c_{2} \varepsilon^{m}+\frac{1}{2} c_{1} \varepsilon^{m}=c \varepsilon^{m}, \forall \varepsilon \in \mathbf{I}_{\eta}, \forall x \in K .
$$

Portanto, $\left\|\partial^{\alpha} f_{\varepsilon}(\cdot)\right\|_{K} \leq c \varepsilon^{m}$, para todo $\varepsilon \in \mathbf{I}_{\eta}$.

Seja $\Omega$ um subconjunto aberto de $\mathbb{R}^{n}$. Então a aplicação $g: \mathscr{C}^{\infty}(\Omega) \longrightarrow \mathcal{G}(\Omega)$ dada por $g(f):=\left[\left(f_{\varepsilon}\right)_{\varepsilon}\right]$, onde $f_{\varepsilon}:=f$, para todo $\varepsilon \in \mathbf{I}$, é um homomorfismo injetor de anéis. Assim, identificando $\mathscr{C}^{\infty}(\Omega) \operatorname{com} g\left(\mathscr{C}^{\infty}(\Omega)\right.$ ), podemos escrever $\mathscr{C}^{\infty}(\Omega) \subset \mathcal{G}(\Omega)$ ([Sil-A, Seção 1.3]).

A Proposição a seguir nos permitirá identificar $\overline{\mathbb{K}}$ com um subconjunto de $\mathcal{G}(\Omega)$ e, assim, poderemos escrever $\bar{K} \subset \mathcal{G}(\Omega)$.

Proposição 1.5.7 Seja $\Omega$ um subconjunto aberto de $\mathbb{R}^{n}$. A aplicação $u: \bar{K} \longrightarrow \mathcal{G}(\Omega)$, dada por $u(\lambda):=\left[\left(\lambda_{\varepsilon}^{*}\right)_{\varepsilon}\right]$, onde $\left(\lambda_{\varepsilon}\right)_{\varepsilon}$ é um representante de $\lambda$ e $\lambda_{\varepsilon}^{*}(x):=\lambda_{\varepsilon}$, para todo $\varepsilon \in \mathbf{I}$ e todo $x \in \Omega$, é um homomorfismo injetor de anéis.

Demonstração: Ver [Sil-A, Proposição 1.8.1].

Definição 1.5.8 Sejam $\Omega$ um subconjunto aberto de $\mathbb{R}^{n}$ e $f \in \mathcal{G}(\Omega)$. Diz-se que $f$ é constante se $f \in u(\overline{\mathbb{K}})$, onde u é como na Proposição 1.5.7. 
Sejam $\Omega$ um subconjunto aberto de $\mathbb{R}^{n}$ e $\alpha \in \mathbb{N}^{n}$. Observamos que a aplicação $\partial^{\alpha}: \mathcal{E}_{M}[\Omega] \longrightarrow \mathcal{E}_{M}[\Omega]$, dada por $\partial^{\alpha}\left(\left(f_{\varepsilon}\right)_{\varepsilon}\right):=\left(\partial^{\alpha} f_{\varepsilon}\right)_{\varepsilon}$, está bem definida e existe uma única aplicação $\mathbb{K}$-linear de $\mathcal{G}(\Omega)$ em $\mathcal{G}(\Omega)$, também denotada por $\partial^{\alpha}$, que torna comutativo o diagrama

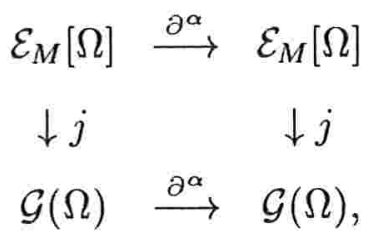

onde $j$ é a projeção canônica ([Sil-A, Seção 1.2]).

Em razão do exposto acima, faz sentido a definição dada a seguir.

Definição 1.5.9 Sejam $\Omega$ um subconjunto aberto de $\mathbb{R}^{n}$ e $f \in \mathcal{G}(\Omega)$. Para cada $\alpha \in \mathbb{N}^{n}$, chama-se de derivada parcial de ordem $\alpha$ de $f$, e denota-se por $\partial^{\alpha} f$, o elemento de $\mathcal{G}(\Omega)$ dado por $\partial^{\alpha} f:=\left[\left(\partial^{\alpha} f_{\varepsilon}\right)_{\varepsilon}\right]$, onde $\left(f_{\varepsilon}\right)_{\varepsilon}$ é um representante qualquer de $f$.

Proposição 1.5.10 Sejam $\Omega$ um subconjunto aberto de $\mathbb{R}^{n}$ e $\alpha \in \mathbb{N}^{n}$. Então

$$
\partial^{\alpha}(f g)=\sum_{0 \leq \beta \leq \alpha}\left(\begin{array}{l}
\alpha \\
\beta
\end{array}\right)\left(\partial^{\beta} f\right)\left(\partial^{\alpha-\beta} g\right),
$$

quaisquer que sejam $f, g \in \mathcal{G}(\Omega)$.

Demonstração: Ver [Sil-A, Proposição 1.2.3].

É fácil verificar que, se $f \in \mathcal{G}(\Omega ; \mathbb{R})$ e $\left(f_{1 \varepsilon}\right)_{\varepsilon}$ e $\left(f_{2 \varepsilon}\right)_{\varepsilon}$ são representantes de $f$, então $\left(\partial_{i} f_{1 \varepsilon}-\partial_{i} f_{2 \varepsilon}\right)_{\varepsilon} \in \mathcal{N}[\Omega ; \mathbb{R}]$, para todo $1 \leq i \leq n$, onde um elemento arbitrário de $\mathbb{R}^{n}$ é denotado por $\left(x_{1}, \ldots, x_{n}\right)$ e $\partial_{i}:=\frac{\partial}{\partial x_{i}}$, para todo $1 \leq i \leq n$. Portanto, podemos definir $\nabla f$ (gradiente de $f$ ) como na Definição a seguir. 
Definição 1.5.11 Sejam $\Omega$ um subconjunto aberto de $\mathbb{R}^{n}$. Para cada $f \in \mathcal{G}(\Omega ; \mathbb{R})$, chamase de gradiente de $f$, e denota-se por $\nabla f$, o elemento de $(\mathcal{G}(\Omega ; \mathbb{R}))^{n}$ dado por

$$
\nabla f:=\left(\left[\left(\partial_{1} f_{\varepsilon}\right)_{\varepsilon}\right], \ldots,\left[\left(\partial_{n} f_{\varepsilon}\right)_{\varepsilon}\right]\right)
$$

onde $\left(f_{\varepsilon}\right)_{\varepsilon}$ é um representante qualquer de $f$, um elemento arbitrário de $\mathbb{R}^{n}$ é denotado por $\left(x_{1}, \ldots, x_{n}\right)$ e $\partial_{i}:=\frac{\partial}{\partial x_{i}}$, para todo $1 \leq i \leq n$.

O próximo resultado é análogo ao do contexto clássico.

Proposição 1.5.12 Sejam $\Omega$ um subconjunto aberto conexo de $\mathbb{R}^{n}$ e $f \in \mathcal{G}(\Omega ; \mathbb{R})$. Então $\nabla f=0$ se, e somente se, $f$ é constante.

Demonstração: Seja $\left(f_{\varepsilon}\right)_{\varepsilon}$ um representante de $f$.

Suponhamos, primeiramente, que $\nabla f=0$. Logo, temos que $\left(\partial_{i} f_{\varepsilon}\right)_{\varepsilon} \in \mathcal{N}[\Omega ; \mathbb{R}]$, para todo $1 \leq i \leq n$.

Pela Definição 1.5.8, é suficiente provarmos que existe $\lambda \in \overline{\mathbb{R}}$ tal que $\left(f_{\varepsilon}-\lambda_{\varepsilon}^{*}\right)_{\varepsilon} \in$ $\mathcal{N}[\Omega ; \mathbb{R}]$, onde $\left(\lambda_{\varepsilon}\right)_{\varepsilon}$ é um representante de $\lambda$ e $\lambda_{\varepsilon}^{*}(x):=\lambda_{\varepsilon}$, para todo $\varepsilon \in \mathbf{I}$ e todo $x \in \Omega$.

Seja $x_{0} \in \Omega$ e consideremos $\lambda_{\varepsilon}:=f_{\varepsilon}\left(x_{0}\right)$ e $\lambda_{\varepsilon}^{*}(x)=\lambda_{\varepsilon}$, para todo $\varepsilon \in \mathbf{I}$ e todo $x \in \Omega$. É claro que $\left(\lambda_{\varepsilon}\right)_{\varepsilon} \in \mathcal{E}_{M}(\mathbb{R})$ e que $\left(f_{\varepsilon}-\lambda_{\varepsilon}^{*}\right)_{\varepsilon} \in \mathcal{E}_{M}[\Omega ; \mathbb{R}]$. Provaremos que $\left(f_{\varepsilon}-\lambda_{\varepsilon}^{*}\right)_{\varepsilon} \in \mathcal{N}[\Omega ; \mathbb{R}]$. Assim, $f=u(\lambda)$, onde $\lambda:=\left[\left(\lambda_{\varepsilon}\right)_{\varepsilon}\right] \in \bar{R}$ e $u$ é como na Proposição 1.5.7.

Sejam $K \subset \subset \Omega$ e $q \in \mathbb{N}$. Da compacidade de $K$ existem $x^{1}, \ldots, x^{p} \in K$ e $r_{1}, \ldots, r_{p}>0$ tais que

$$
K \subset \bigcup_{t=1}^{p} \overline{B_{r_{t}}\left(x^{t}\right)} \subset \subset \Omega .
$$

Da conexidade de $\Omega$, existe, para cada $1 \leq t \leq p$, uma poligonal $L_{t} \subset \Omega$ que une $x^{t}$ a $x_{0}$. Consideremos

$$
W:=\bigcup_{t=1}^{p}\left(\overline{B_{r_{t}}\left(x^{t}\right)} \cup L_{t}\right) \subset \subset \Omega
$$


e denotemos, para cada $1 \leq t \leq p$, por $v_{t}$ o número de vértices de $L_{t}$, onde cada vértice de $L_{t}$ é denotado por $x_{j t}$ com $1 \leq j \leq v_{t}$, onde $x_{1 t}=x^{t}$ e $x_{v_{t} t}=x_{0}$.

Seja $x \in K$. Então existe $1 \leq t \leq p$ tal que $x \in \overline{B_{r_{t}}\left(x^{t}\right)}$ e, portanto, como a poligonal $L_{t} \subset \Omega$, temos, para $\varepsilon \in \mathbf{I}$, que

$$
\left|f_{\varepsilon}(x)-\lambda_{\varepsilon}^{*}(x)\right|=\left|f_{\varepsilon}(x)-f_{\varepsilon}\left(x_{0}\right)\right| \leq \sum_{j=1}^{v_{t}}\left|f_{\varepsilon}\left(x_{(j-1) t}\right)-f_{\varepsilon}\left(x_{j t}\right)\right|,
$$

onde $x_{0 t}=x$. Notemos que $f_{\varepsilon}$ é diferenciável, para todo $\varepsilon \in \mathbf{I}$, pois $\left(f_{\varepsilon}\right)_{\varepsilon} \in\left(\mathscr{C}^{\infty}(\Omega ; \mathbb{R})\right)^{\mathbf{I}}$. Assim, para cada $\varepsilon \in \mathbf{I}$ e cada $1 \leq j \leq v_{t}$ fixados, existe, pelo Teorema do Valor Médio, $c_{j t \varepsilon}$ pertencente ao segmento de extremidades $x_{(j-1) t}$ e $x_{j t}$, o qual está contido em $W$, tal que

$$
\sum_{j=1}^{v_{t}}\left|f_{\varepsilon}\left(x_{(j-1) t}\right)-f_{\varepsilon}\left(x_{j t}\right)\right| \leq \sum_{j=1}^{v_{t}}\left|\nabla f_{\varepsilon}\left(c_{j t \varepsilon}\right)\right|\left|x_{(j-1) t}-x_{j t}\right|
$$

e, portanto,

$$
\left|f_{\varepsilon}(x)-\lambda_{\varepsilon}^{*}(x)\right| \leq \sum_{j=1}^{v_{t}}\left|\nabla f_{\varepsilon}\left(c_{j t \varepsilon}\right)\right|\left|x_{(j-1) t}-x_{j t}\right| \leq \sum_{j=1}^{v_{t}}\left|\nabla f_{\varepsilon}\left(c_{j t \varepsilon}\right)\right|\left(\left|x_{(j-1) t}\right|+\left|x_{j t}\right|\right) .
$$

Como $\left(\partial_{i} f_{\varepsilon}\right)_{\varepsilon} \in \mathcal{N}[\Omega ; \mathbb{R}]$, para todo $1 \leq i \leq n$, pela Proposição $1.5 .5(2)$, existem $c_{i}>0$ e $\eta_{i} \in \mathbf{I}$ tais que

$$
\left\|\partial_{i} f_{\varepsilon}(\cdot)\right\|_{W} \leq c_{i} \varepsilon^{q}, \forall \varepsilon \in \mathbf{I}_{\eta_{i}} .
$$

Sejam $\eta:=\min \left\{\eta_{i}: 1 \leq i \leq n\right\}, d:=\max \left\{c_{i}: 1 \leq i \leq n\right\}$ e $M>0$ tal que $|y| \leq M$, para todo $y \in W$. Então

$$
\left|f_{\varepsilon}(x)-\lambda_{\varepsilon}^{*}(x)\right| \leq 2 M v_{t} n d \varepsilon^{q}, \forall \varepsilon \in \mathbf{I}_{\eta} .
$$

Consideremos $s=\max \left\{v_{t}: 1 \leq t \leq p\right\}$ e $c:=2 M$ snd. Então temos que

$$
\left|f_{\varepsilon}(x)-\lambda_{\varepsilon}^{*}(x)\right| \leq c \varepsilon^{q}, \forall \varepsilon \in \mathbf{I}_{\eta}, \forall x \in K,
$$

ou seja,

$$
\left\|\left(f_{\varepsilon}-\lambda_{\varepsilon}^{*}\right)(\cdot)\right\|_{K} \leq c \varepsilon^{q}, \forall \varepsilon \in \mathbf{I}_{\eta}
$$

Portanto, pelo Teorema 1.5.6, obtemos que $\left(f_{\varepsilon}-\lambda_{\varepsilon}^{*}\right)_{\varepsilon} \in \mathcal{N}[\Omega ; \mathbb{I R}]$. 
Reciprocamente, suponhamos que $f$ é constante. Assim, existe $\lambda:=\left[\left(\lambda_{\varepsilon}\right)_{\varepsilon}\right] \in \mathbb{R}$ tal que $f=\left[\left(\lambda_{\varepsilon}^{*}\right)_{\varepsilon}\right]$, onde $\lambda_{\varepsilon}^{*}(x):=\lambda_{\varepsilon}$, para todo $\varepsilon \in \mathbf{I}$ e todo $x \in \Omega$. Logo,

$$
\partial_{i} \lambda_{\varepsilon}^{*}(x)=0, \forall 1 \leq i \leq n, \forall \varepsilon \in \mathbf{I}, \forall x \in \Omega
$$

e, assim, $\left(\partial_{i} \lambda_{\varepsilon}^{*}\right)_{\varepsilon} \in \mathcal{N}[\Omega ; \mathbb{R}]$, para todo $1 \leq i \leq n$. Portanto, $\nabla f=0$

Sejam $\Omega$ um subconjunto aberto de $\mathbb{R}^{n}, f \in \mathcal{G}(\Omega)$ e $x \in \Omega$. A seguir, iremos apresentar o valor pontual de $f$ em $x$. Para isso, convém observarmos que, se $\left(f_{1 \varepsilon}\right)_{\varepsilon}$ e $\left(f_{2 \varepsilon}\right)_{\varepsilon}$ são representantes de $f$, então $\left(f_{1 \varepsilon}(x)-f_{2 \varepsilon}(x)\right)_{\varepsilon} \in \mathcal{N}(\mathbb{K})$.

Definição 1.5.13 Sejam $\Omega$ um subconjunto aberto de $\mathbb{R}^{n}$ e $f \in \mathcal{G}(\Omega)$. Para cada $x \in \Omega$, chama-se de valor pontual de $f$ em $x$, e denota-se por $f_{x}$, o elemento de $\overline{\mathbb{K}}$ dado por $f_{x}:=\left[\left(f_{\varepsilon}(x)\right)_{\varepsilon}\right]$, onde $\left(f_{\varepsilon}\right)_{\varepsilon}$ é um representante qualquer de $f$.

Exemplo 1.5.14 Seja, para cada $\varepsilon \in \mathbf{I}, f_{\varepsilon}: \mathbb{R} \longrightarrow \mathbb{R}$, dada por $f_{\varepsilon}(x):=x e^{-x^{2} / \varepsilon}$. Então tem-se que

1. $\left(f_{\varepsilon}\right)_{\varepsilon} \in \mathcal{E}_{M}[\mathbb{R} ; \mathbb{R}]$

2. se $f:=\left[\left(f_{\varepsilon}\right)_{\varepsilon}\right]$, então $f_{x}=0$ em $\bar{R}$, para todo $x \in \mathbb{R}$, e $f \neq 0$ em $\mathcal{G}(\mathbb{R} ; \mathbb{R})$.

De fato, primeiramente, observemos que $\left(f_{\varepsilon}\right)_{\varepsilon} \in\left(\mathscr{C}^{\infty}(\Omega ; \mathbb{K})\right)^{\mathbf{I}}$ e que, para cada $\alpha \in \mathbb{I}$ fixado,

$$
f_{\varepsilon}^{(\alpha)}(x)=e^{-x^{2} / \varepsilon} \sum_{j=0}^{\alpha} \frac{1}{\varepsilon^{j}} p_{j}(x), \forall \varepsilon \in \mathbf{I},
$$

onde $p_{j}$ é um polinômio em $x$ de grau menor ou igual a $\alpha$, para todo $0 \leq j \leq \alpha$. Seja $K \subset \subset \mathbb{R}$. Como $p_{j}$ é uma função contínua, existe $M_{j}:=\max \left\{p_{j}(x): x \in K\right\}$, para todo $0 \leq j \leq \alpha$. Dessa forma,

$$
\left|f_{\varepsilon}^{(\alpha)}(x)\right| \leq e^{-x^{2} / \varepsilon} \sum_{j=0}^{\alpha} \frac{1}{\varepsilon^{j}}\left|M_{j}\right| \leq \sum_{j=0}^{\alpha} \frac{1}{\varepsilon^{j}}\left|M_{j}\right|, \forall \varepsilon \in \mathbf{I}, \forall x \in K,
$$


e, como $\frac{1}{\varepsilon^{j}} \leq \frac{1}{\varepsilon^{\alpha}}$, para todo $0 \leq j \leq \alpha$ (pois, $0<\varepsilon \leq 1$ ), temos

$$
\left|f_{\varepsilon}^{(\alpha)}(x)\right| \leq \frac{1}{\varepsilon^{\alpha}} \sum_{j=0}^{\alpha}\left|M_{j}\right|, \forall \varepsilon \in \mathbf{I}, \forall x \in K .
$$

Seja $c:=\sum_{j=0}^{\alpha}\left|M_{j}\right|+1$. Então

$$
\left\|f_{\varepsilon}^{(\alpha)}(\cdot)\right\|_{K} \leq c \varepsilon^{-\alpha}, \forall \varepsilon \in \mathbf{I}
$$

e, portanto, pela Proposição 1.5.5 (1), $\left(f_{\varepsilon}\right)_{\varepsilon} \in \mathcal{E}_{M}[\mathbb{R} ; \mathbb{R}]$, o que prova 1 .. Notemos que, se $x \in \mathbb{R}$ e $q \in \mathbb{N}$, então

$$
\lim _{\varepsilon \downarrow 0} \frac{\left|f_{\varepsilon}(x)\right|}{\varepsilon^{q}}=\lim _{\varepsilon \downarrow 0}\left|\frac{x}{e^{x^{2} / \varepsilon}} \frac{1}{\varepsilon^{q}}\right|=\lim _{\varepsilon \downarrow 0}\left|\frac{x / \varepsilon^{q}}{e^{x^{2} / \varepsilon}}\right|=0
$$

e, portanto, $\left(f_{\varepsilon}(x)\right)_{\varepsilon} \in \mathcal{N}(\mathbb{R})$. Logo, $f_{x}=0$, para todo $x \in \mathbb{R}$. Observemos que

$$
f_{\varepsilon}^{(1)}(x)=e^{-x^{2} / \varepsilon}-\frac{2 x^{2} e^{-x^{2} / \varepsilon}}{\varepsilon} .
$$

Logo, para $K:=\{0\} \subset \subset \mathbb{R}$ e $q=1$, temos

$$
\lim _{\varepsilon \downarrow 0} \frac{\left\|f_{\varepsilon}^{(1)}(\cdot)\right\|}{\varepsilon}=\lim _{\varepsilon \downarrow 0} \frac{1}{\varepsilon}=+\infty
$$

e, portanto, $\left(f_{\varepsilon}\right)_{\varepsilon} \notin \mathcal{N}[\mathbb{R} ; \mathbb{R}]$, ou seja, $f \neq 0$.

O Exemplo 1.5.14 nos mostra que uma função generalizada não é definida por seu valor pontual. Esse problema será resolvido na próxima seção.

\subsection{O conjunto $\widetilde{\Omega}_{c}$ e o valor pontual generalizado}

Vimos, no Exemplo 1.5.14, que uma função generalizada, diferentemente do que ocorre no contexto clássico, não é definida por seu valor pontual. Motivados por esse problema, M. Kunzinger e M. Oberguggenberger $([\mathrm{KO}])$, introduziram o conjunto $\widetilde{\Omega}_{c}$ e a definição de valor 
pontual generalizado. A partir desses conceitos, provaram que uma função generalizada é definida por seu valor pontual generalizado. Esse resultado e algumas propriedades interessantes do conjunto $\widetilde{\Omega}_{c}$, devido a J. Aragona, R. Fernandez e S. O. Juriaans, serão apresentados nesta seção.

Definição 1.6.1 Seja $\Omega$ um subconjunto aberto de $\mathbb{K}^{n}$. Define-se

$$
\Omega_{M}:=\left\{\left(x_{\varepsilon}\right)_{\varepsilon} \in \Omega^{\mathbf{I}}: \exists N \in \mathbb{N}, \exists c>0 e \exists \eta \in \mathbf{I} \operatorname{com}\left|x_{\varepsilon}\right| \leq c \varepsilon^{-N}, \forall \varepsilon \in \mathbf{I}_{\eta}\right\}
$$

$e$

$$
\Omega_{c}:=\left\{\left(x_{\varepsilon}\right)_{\varepsilon} \in \Omega^{\mathbf{I}}: \exists K \subset \subset \Omega e \exists \eta \in \mathbf{I} \operatorname{com} x_{\varepsilon} \in K, \forall \varepsilon \in \mathbf{I}_{\eta}\right\}
$$

É claro que $\Omega_{M} \subset\left(\mathcal{E}_{M}(\mathbb{K})\right)^{n}$ e $\Omega_{c} \subset \Omega_{M}$.

Definição 1.6.2 Seja $\Omega$ um subconjunto aberto de $\mathbb{K}^{n}$. Diz-se que $\left(x_{\varepsilon}\right)_{\varepsilon},\left(y_{\varepsilon}\right)_{\varepsilon} \in \Omega_{M}$ são equivalentes, e denota-se por $\left(x_{\varepsilon}\right)_{\varepsilon} \sim\left(y_{\varepsilon}\right)_{\varepsilon}$, se, e somente se,

$$
\forall q \in \mathbb{N}, \exists c>0, \exists \eta \in \mathbf{I} \text { tais que }\left|x_{\varepsilon}-y_{\varepsilon}\right| \leq c \varepsilon^{q}, \forall \varepsilon \in \mathbf{I}_{\eta}
$$

Notamos que $\sim$ é uma relação de equivalência sobre $\Omega_{M}$.

Seja $\Omega$ um subconjunto aberto de $\mathbb{K}^{n}$. Se $\left(x_{\varepsilon}\right)_{\varepsilon} \in \Omega_{M}$, então definimos

$$
\left(x_{\varepsilon}\right)_{\varepsilon}^{\wedge}:=\left\{\left(y_{\varepsilon}\right)_{\varepsilon} \in \Omega_{M}:\left(x_{\varepsilon}\right)_{\varepsilon} \sim\left(y_{\varepsilon}\right)_{\varepsilon}\right\}
$$

Observamos que, se $\left(x_{\varepsilon}\right)_{\varepsilon}:=\left(\left(x_{1 \varepsilon}, \ldots, x_{n \varepsilon}\right)\right)_{\varepsilon},\left(y_{\varepsilon}\right)_{\varepsilon}:=\left(\left(y_{1 \varepsilon}, \ldots, y_{n \varepsilon}\right)\right)_{\varepsilon} \in \Omega_{M}$, então $\left(x_{\varepsilon}\right)_{\varepsilon} \sim\left(y_{\varepsilon}\right)_{\varepsilon}$ se, e somente se, $\left(x_{i \varepsilon}-y_{i \varepsilon}\right)_{\varepsilon} \in \mathcal{N}(\mathbb{K})$, para todo $1 \leq i \leq n$. Portanto, podemos definir a função

$$
\begin{array}{ccc}
h: \quad \Omega_{M} / \sim & \longrightarrow & \overline{\mathbb{K}}^{n} \\
\left(\left(x_{1 \varepsilon}, \ldots, x_{n \varepsilon}\right)\right)_{\varepsilon}^{\wedge} & \longmapsto & \left(\left[\left(x_{1 \varepsilon}\right)_{\varepsilon}\right], \ldots,\left[\left(x_{n \varepsilon}\right)_{\varepsilon}\right]\right)
\end{array}
$$


que é uma função injetora.

A partir da função $h$ iremos, a seguir, definir os conjuntos $\widetilde{\Omega}$ e $\widetilde{\Omega}_{c}$.

Definição 1.6.3 Seja $\Omega$ um subconjunto aberto de $\mathbb{K}^{n}$. Define-se

$$
\widetilde{\Omega}:=h\left(\Omega_{M} / \sim\right) \quad e \quad \widetilde{\Omega}_{c}:=h\left(\left\{\left(x_{\varepsilon}\right)_{\varepsilon}^{\wedge}:\left(x_{\varepsilon}\right)_{\varepsilon} \in \Omega_{c}\right\}\right)
$$

onde $h$ é definida em (1.11).

Os conjuntos $\Omega_{M} / \sim \mathrm{e}\left\{\left(x_{\varepsilon}\right)_{\varepsilon}^{\wedge}:\left(x_{\varepsilon}\right)_{\varepsilon} \in \Omega_{c}\right\}$ foram introduzidos por M. Kunzinger e M. Oberguggenberger e foram denotados por $\widetilde{\Omega}$ e $\tilde{\Omega}_{c}$, respectivamente. Contudo, como trabalharemos com as imagens desses conjuntos pela função $h$, optamos por denotar as suas imagens por $\widetilde{\Omega}$ e $\widetilde{\Omega}_{c}$, respectivamente (Definição 1.6.3).

Um elemento de $\widetilde{\Omega}$ é chamado de ponto generalizado e um elemento de $\widetilde{\Omega}_{c}$ é chamado de ponto compactamente suportado. Observamos que $\widetilde{\Omega} \subset \overline{\mathbb{K}}^{n}$ e, se $\Omega=\mathbb{K}^{n}$, então $\widetilde{\Omega}=\overline{\mathbb{K}}^{n}$.

Uma propriedade importante dos elementos de $\Omega_{c}$, que surgiu a partir de nossos estudos do artigo [AFJ-1], é o seguinte resultado:

Proposição 1.6.4 Sejam $\Omega$ um subconjunto aberto de $\mathbb{K}^{n}$ e $\left(x_{\varepsilon}\right)_{\varepsilon} \in \Omega_{c}$. Tem-se que:

1. se $\left(y_{\varepsilon}\right)_{\varepsilon} \in\left(\mathbb{K}^{n}\right)^{\mathbf{I}}$ e $\lim _{\varepsilon \downarrow 0}\left|x_{\varepsilon}-y_{\varepsilon}\right|=0$, então existem $K_{1} \subset \subset \Omega$ e $\eta_{1} \in \mathbf{I}$ tais que $o$ segmento de extremidades $x_{\varepsilon}$ e $y_{\varepsilon}$ está contido em $K_{1}$, para todo $\varepsilon \in \mathbf{I}_{\eta_{1}}$. Em particular, tem-se que $x_{\varepsilon}, y_{\varepsilon} \in K_{1}$, para todo $\varepsilon \in \mathbf{I}_{\eta_{1}}$;

2. se $\Omega$ é convexo e $\left(y_{\varepsilon}\right)_{\varepsilon} \in \Omega_{c}$, então existem $K_{2} \subset \subset \Omega$ e $\eta_{2} \in \mathbf{I}$ tais que o segmento de extremidades $x_{\varepsilon}$ e $y_{\varepsilon}$ está contido em $K_{2}$, para todo $\varepsilon \in \mathbf{I}_{\eta_{2}}$. Em particular, tem-se que $x_{\varepsilon}, y_{\varepsilon} \in K_{2}$, para todo $\varepsilon \in \mathbf{I}_{\eta_{2}}$. 
Demonstração: Como todo aberto de $\mathbb{C}^{n}$ é homeomorfo a um aberto de $\mathbb{R}^{2 n}$, podemos supor, sem perda de generalidade, que $\mathbb{K}=\mathbb{R}$.

De $\left(x_{\varepsilon}\right)_{\varepsilon} \in \Omega_{c}$, existem $L_{1} \subset \subset \Omega$ e $\nu_{1} \in \mathbf{I}$ tais que $x_{\varepsilon} \in L_{1}$, para todo $\varepsilon \in \mathbf{I}_{\nu_{1}}$.

Consideremos $V$ um subconjunto aberto de $\Omega$ tal que $L_{1} \subset V \subset \bar{V} \subset \subset \Omega$ e seja $r:=\frac{1}{2} \operatorname{dist}\left(L_{1}, V^{c}\right)>0$. Então,

$$
B_{r}\left(x_{\varepsilon}\right) \subset V \subset \bar{V} \subset \subset \Omega, \forall \varepsilon \in \mathbf{I}_{\nu_{1}} \text {. }
$$

Seja $K_{1}:=\bar{V} \subset \subset \Omega . \operatorname{Se}\left(y_{\varepsilon}\right)_{\varepsilon} \in\left(\mathbb{K}^{n}\right)^{\mathbf{I}}$ e $\lim _{\varepsilon \downarrow 0}\left|x_{\varepsilon}-y_{\varepsilon}\right|=0$, existe $\nu_{2} \in \mathbf{I}$ tal que

$$
\left|x_{\varepsilon}-y_{\varepsilon}\right|<r, \forall \varepsilon \in \mathbf{I}_{\nu_{2}} \text {. }
$$

Portanto, para $\eta_{1}:=\min \left\{\nu_{1}, \nu_{2}\right\}$ e $\varepsilon \in \mathbf{I}_{\eta_{1}}$, temos, por (1.12) e (1.13), que $x_{\varepsilon}, y_{\varepsilon} \in B_{r}\left(x_{\varepsilon}\right) \subset$ $\bar{V}=K_{1}$ e, assim, o segmento de extremidades $x_{\varepsilon}$ e $y_{\varepsilon}$ está contido em $B_{r}\left(x_{\varepsilon}\right) \subset K_{1}$, o que prova 1 ..

Se $\left(y_{\varepsilon}\right)_{\varepsilon} \in \Omega_{c}$, existem $L_{2} \subset \subset \Omega$ e $\nu_{3} \in \mathbf{I}$ tais que $y_{\varepsilon} \in L_{2}$, para todo $\varepsilon \in \mathbf{I}_{\nu_{3}}$.

Sejam $K_{2}$ a envoltória convexa de $L_{1} \cup L_{2}$ e $\eta_{2}:=\min \left\{\nu_{1}, \nu_{3}\right\}$. Então $K_{2} \subset \subset \mathbb{R}^{n}$ e, para $\varepsilon \in \mathbf{I}_{\eta_{2}}$, temos que $x_{\varepsilon}, y_{\varepsilon} \in K_{2}$ e, como $K_{2}$ é convexo, segue que o segmento de extremidades $x_{\varepsilon}$ e $y_{\varepsilon}$ está contido em $K_{2}$. Para concluirmos 2., basta observarmos que, se $\Omega$ é convexo, então $K_{2} \subset \Omega$.

Para a próxima definição, convém observarmos que, se $\Omega$ é um subconjunto aberto de $\mathbb{R}^{n}, f \in \mathcal{G}(\Omega),\left(f_{\varepsilon}\right)_{\varepsilon}$ e $\left(g_{\varepsilon}\right)_{\varepsilon}$ são representantes de $f$ e $\left(x_{\varepsilon}\right)_{\varepsilon}:=\left(\left(x_{1 \varepsilon}, \ldots, x_{n \varepsilon}\right)\right)_{\varepsilon},\left(y_{\varepsilon}\right)_{\varepsilon}:=$ $\left(\left(y_{1 \varepsilon}, \ldots, y_{n \varepsilon}\right)\right)_{\varepsilon}$ são elementos de $\Omega_{M}$ tais que $\left(x_{\varepsilon}\right)_{\varepsilon} \in \Omega_{c}$ e $\left(x_{i \varepsilon}-y_{i \varepsilon}\right)_{\varepsilon} \in \mathcal{N}(\mathbb{K})$, para todo $1 \leq i \leq n$, então $\left(y_{\varepsilon}\right)_{\varepsilon} \in \Omega_{c}$ e $\left(f_{\varepsilon}\left(x_{\varepsilon}\right)-g_{\varepsilon}\left(y_{\varepsilon}\right)\right)_{\varepsilon} \in \mathcal{N}(\mathbb{K})$ (Proposição 1.6.4 (1) e [Sil-A, Proposição 1.10.3]).

Definição 1.6.5 Sejam $\Omega$ um subconjunto aberto de $\mathbb{R}^{n}$ e $f \in \mathcal{G}(\Omega)$. Para cada $x \in \widetilde{\Omega}_{c}$, chama-se de valor pontual generalizado de $f$ em $x$, e denota-se por $f(x)$, o elemento de $\overline{\mathbb{I}}$ dado por

$$
f(x):=\left[\left(f_{\varepsilon}\left(x_{\varepsilon}\right)\right)_{\varepsilon}\right]
$$


onde $\left(f_{\varepsilon}\right)_{\varepsilon}$ é um representante qualquer de $f$ e $\left(x_{\varepsilon}\right)_{\varepsilon}:=\left(\left(x_{1 \varepsilon}, \ldots, x_{n \varepsilon}\right)\right)_{\varepsilon}$ é um elemento qualquer de $\Omega_{M}$ tal que $\left(x_{\varepsilon}\right)_{\varepsilon} \in \Omega_{c}$ e $x=\left(\left[\left(x_{1 \varepsilon}\right)_{\varepsilon}\right], \ldots,\left[\left(x_{n \varepsilon}\right)_{\varepsilon}\right]\right)$.

A prova do próximo resultado pode ser encontrada em [KO] e em [Sil-A]. No entanto, apresentaremos uma demonstração mais simples, encontrada em [GKOS], a qual utiliza o Teorema 1.5.6.

Teorema 1.6.6 Sejam $\Omega$ um subconjunto aberto de $\mathbb{R}^{n}$ e $f \in \mathcal{G}(\Omega)$. Então $f=0$ em $\mathcal{G}(\Omega)$ se, e somente se, $f(x)=0$ em $\overline{\mathbb{K}}$, para todo $x \in \widetilde{\Omega}_{c}$.

Demonstração: Seja $\left(f_{\varepsilon}\right)_{\varepsilon}$ um representante de $f$.

Primeiramente, suponhamos que $f=0$. Sejam $x \in \widetilde{\Omega}_{c}$ e $\left(x_{\varepsilon}\right)_{\varepsilon}:=\left(\left(x_{1 \varepsilon}, \ldots, x_{n \varepsilon}\right)\right)_{\varepsilon} \in \Omega_{c}$ tal que $x=\left(\left[\left(x_{1 \varepsilon}\right)_{\varepsilon}\right], \ldots,\left[\left(x_{n \varepsilon}\right)_{\varepsilon}\right]\right)$. Então existem $K \subset \subset \Omega$ e $\eta_{1} \in \mathbf{I}$ tais que $x_{\varepsilon} \in K$, para todo $\varepsilon \in \mathbf{I}_{\eta_{1}}$.

Seja $q \in \mathbb{N}$. Como $\left(f_{\varepsilon}\right)_{\varepsilon} \in \mathcal{N}[\Omega]$, temos, pela Proposição 1.5.5 (2), que existem $c>0$ e $\eta_{2} \in \mathbf{I}$ tais que

$$
\left\|f_{\varepsilon}(\cdot)\right\|_{K} \leq c \varepsilon^{q}, \forall \varepsilon \in \mathbf{I}_{\eta_{2}} .
$$

Consideremos $\eta:=\min \left\{\eta_{1}, \eta_{2}\right\}$. Logo,

$$
\left|f_{\varepsilon}\left(x_{\varepsilon}\right)\right| \leq c \varepsilon^{q}, \forall \varepsilon \in \mathbf{I}_{\eta}
$$

e, portanto, $\left(f_{\varepsilon}\left(x_{\varepsilon}\right)\right)_{\varepsilon} \in \mathcal{N}(\mathbb{K})$ (Proposição 1.1.5), ou seja, $f(x)=0 \mathrm{em} \bar{K}$.

Reciprocamente, suponhamos que $f \neq 0 \mathrm{em} \mathcal{G}(\Omega)$. Então, pelo Teorema 1.5.6, existem $K \subset \subset \Omega$ e $m \in \mathbb{N}$ tais que para todo $\eta \in \mathbf{I}$ existe $\varepsilon \in \mathbf{I}_{\eta}$ satisfazendo

$$
\left\|f_{\varepsilon}(\cdot)\right\|_{K}>\varepsilon^{m}
$$

Seja $\eta_{0} \in \mathbf{I}$ e consideremos $\varepsilon_{0} \in \mathbf{I}_{\eta_{0}}$ satisfazendo (1.14). Para cada $k \in \mathbb{N}^{*}$, seja $\eta_{k}:=\frac{\varepsilon_{k-1}}{2^{k}}$ e consideremos $\varepsilon_{k} \in \mathbf{I}_{\eta_{k}}$ verificando (1.14). Logo,

$\left(\varepsilon_{k}\right)_{k \in N}$ é uma seqüência de elementos de I tal que $\lim _{k \rightarrow \infty} \varepsilon_{k}=0$ e $\varepsilon_{k+1}<\varepsilon_{k}, \forall k \in \mathbb{N}$, 
pois, $0 \leq \varepsilon_{k} \leq \eta_{k} \leq \frac{1}{2^{k}}$. Além disso, como $\left|f_{\varepsilon}(\cdot)\right|$ é contínua, para todo $\varepsilon \in \mathbf{I}$, e $K \subset \subset \Omega$ temos que $\left|f_{\varepsilon_{k}}(\cdot)\right|$ assume máximo em $z_{k} \in K$, para todo $k \in \mathbb{N}$. Assim, por (1.14),

$$
\left|f_{\varepsilon_{k}}\left(z_{k}\right)\right|=\left\|f_{\varepsilon_{k}}(\cdot)\right\|_{K}>\varepsilon_{k}^{m}, \forall k \in \mathbb{N}
$$

De (1.15), para cada $\varepsilon \in \mathbf{I}$, podemos considerar

$$
x_{\varepsilon}:=\left\{\begin{array}{ll}
z_{0}, & \text { se } \varepsilon_{0}<\varepsilon \leq 1 \\
z_{k} & , \text { se } \varepsilon_{k+1}<\varepsilon \leq \varepsilon_{k}, k \in \mathbb{N}
\end{array} .\right.
$$

Como $K$ é limitado, pois $K \subset \subset \Omega$, existe $r>0$ tal que $\left|z_{k}\right|<r$, para todo $k \in \mathbb{N}$. Logo, $\left|x_{\varepsilon}\right|<r \leq r \varepsilon^{-1}$, para todo $\varepsilon \in \mathbf{I}$, e portanto $\left(x_{\varepsilon}\right)_{\varepsilon}:=\left(\left(x_{1 \varepsilon}, \ldots, x_{n \varepsilon}\right)\right)_{\varepsilon} \in \Omega_{M}$ e, como $x_{\varepsilon} \in K$, para todo $\varepsilon \in \mathbf{I}$, segue que $\left(x_{\varepsilon}\right)_{\varepsilon} \in \Omega_{c}$.

Seja $x:=\left(\left[\left(x_{1 \varepsilon}\right)_{\varepsilon}\right], \ldots,\left[\left(x_{n \varepsilon}\right)_{\varepsilon}\right]\right) \in \widetilde{\Omega}_{c}$. De $(1.16)$, temos que

$$
\left|f_{\varepsilon_{k}}\left(x_{\varepsilon_{k}}\right)\right|=\left|f_{\varepsilon_{k}}\left(z_{k}\right)\right|>\varepsilon_{k}^{m}, \forall k \in \mathbb{N} .
$$

Logo, se existir $\lim _{\varepsilon \downarrow 0} \frac{\left|f_{\varepsilon}\left(x_{\varepsilon}\right)\right|}{\varepsilon^{m}}$ ele não será igual a 0 e, assim, $\left(f_{\varepsilon}\left(x_{\varepsilon}\right)\right)_{\varepsilon} \notin \mathcal{N}(\mathbb{K})$. Portanto, $x \in \widetilde{\Omega}_{c}$ e $f(x) \neq 0$ em $\overline{I K}$.

Do Teorema 1.6.6, tem-se que, se $f, g \in \mathcal{G}(\Omega)$, então

$$
f=g \Longleftrightarrow f(x)=g(x), \forall x \in \tilde{\Omega}_{c},
$$

e, assim, uma função generalizada é definida por seu valor pontual generalizado.

A seguir, apresentaremos algumas propriedades interessantes do conjunto $\widetilde{\Omega}_{c}$, as quais serão úteis no capítulo 4 .

Proposição 1.6.7 Seja $\Omega$ um subconjunto aberto de $\mathbb{K}^{n}$. As seguintes afirmações são verdadeiras:

1. $B_{1}(x) \subset \widetilde{\Omega}_{c} \subset B_{1}^{\prime}(0)$, para todo $x \in \widetilde{\Omega}_{c}$;

2. $\widetilde{\Omega}_{c}$ é um subconjunto aberto de $\overline{\mathbb{K}}^{n}$; 
3. se $x_{0} \in \Omega$ e $V:=\Omega \backslash\left\{x_{0}\right\}$, então $\tilde{V}_{c} \subset \tilde{\Omega}_{c} \backslash B_{1}\left(\tilde{x}_{0}\right) \subset S_{1}\left(\tilde{x}_{0}\right)$, onde $\tilde{x}_{0}:=$ $\left(\left[\left(x_{01 \varepsilon}\right)_{\varepsilon}\right], \ldots,\left[\left(x_{0 n \varepsilon}\right)_{\varepsilon}\right]\right), \operatorname{com}\left(x_{01 \varepsilon}, \ldots, x_{0 n \varepsilon}\right)=x_{0}$, para todo $\varepsilon \in \mathbf{I}$.

Demonstração: Notemos que a afirmação 2. segue diretamente de 1.. Assim, é suficiente provarmos que as afirmações 1 . e 3. são verdadeiras.

Seja $x=\left(x_{1}, \ldots, x_{n}\right):=\left(\left[\left(x_{1 \varepsilon}\right)_{\varepsilon}\right], \ldots,\left[\left(x_{n \varepsilon}\right)_{\varepsilon}\right]\right) \in \widetilde{\Omega}_{c}$, onde $\left(x_{\varepsilon}\right)_{\varepsilon}:=\left(\left(x_{1 \varepsilon}, \ldots, x_{n \varepsilon}\right)\right)_{\varepsilon} \in \Omega_{c}$. Primeiramente, mostraremos que $B_{1}(x) \subset \widetilde{\Omega}_{c}$.

Consideremos $y=\left(y_{1}, \ldots, y_{n}\right):=\left(\left[\left(y_{1 \varepsilon}\right)_{\varepsilon}\right], \ldots,\left[\left(y_{n \varepsilon}\right)_{\varepsilon}\right]\right) \in \overline{\mathbb{K}}^{n}$ tal que $\|y-x\|<1$. Então, pela Proposição 1.4.5 (1)(a), segue que $x \approx y$ e, assim, por (1.2), temos que

$$
\lim _{\varepsilon \downarrow 0}\left|x_{\varepsilon}-y_{\varepsilon}\right|=0
$$

onde $\left(y_{\varepsilon}\right)_{\varepsilon}:=\left(\left(y_{1 \varepsilon}, \ldots, y_{n \varepsilon}\right)\right)_{\varepsilon}$. Portanto, pela Proposição 1.6.4 (1), existem $K \subset \subset \Omega$ e $\eta \in \mathbf{I}$ tais que $y_{\varepsilon} \in K$, para todo $\varepsilon \in \mathbf{I}_{\eta}$.

Seja, para $1 \leq j \leq n$ e para todo $\varepsilon \in \mathbf{I}$,

$$
z_{j \varepsilon}:=\left\{\begin{array}{ll}
y_{j \varepsilon} & , \text { se } 0<\varepsilon<\eta \\
y_{j \frac{\eta}{2}} & , \text { se } \eta \leq \varepsilon \leq 1
\end{array} .\right.
$$

Então $z_{\varepsilon}:=\left(z_{1 \varepsilon}, \ldots, z_{n \varepsilon}\right) \in K$, para todo $\varepsilon \in \mathbf{I} \mathrm{e}$, assim, concluímos que $\left(z_{\varepsilon}\right)_{\varepsilon} \in \Omega_{c} \subset \Omega_{M} \mathrm{e}$ $y=\left(\left[\left(z_{1 \varepsilon}\right)_{\varepsilon}\right], \ldots,\left[\left(z_{n \varepsilon}\right)_{\varepsilon}\right]\right)$. Portanto, $y \in \widetilde{\Omega}_{c}$, o que prova a primeira inclusão de 1 ..

Provaremos, a seguir, que $x \in B_{1}^{\prime}(0)$ e, como $x \in \widetilde{\Omega}_{c}$ é arbitrário, obteremos a segunda inclusão de $1 .$.

Como $\left(x_{\varepsilon}\right)_{\varepsilon} \in \Omega_{c}$, existem $K_{1} \subset \subset \Omega$ e $\eta_{1} \in \mathbf{I}$ tais que $x_{\varepsilon} \in K_{1}$, para todo $\varepsilon \in \mathbf{I}_{\eta_{1}}$.

Da compacidade de $K_{1}$, existe $M>0$ tal que $\left|x_{\varepsilon}\right| \leq M$, para todo $\varepsilon \in \in \mathbf{I}_{\eta_{1}}$, e, assim,

$$
0 \leq \frac{\left|x_{i \varepsilon}\right|}{\varepsilon^{a}} \leq \frac{M}{\varepsilon^{a}}, \forall a \in \mathbb{R}, \forall 1 \leq i \leq n, \forall \varepsilon \in \mathbf{I}_{\eta_{1}},
$$

e, como $\lim _{\varepsilon \downarrow 0} \frac{M}{\varepsilon^{a}}=0$ se, e somente se, $a<0$, concluímos que $x_{i} \alpha_{-a} \approx 0$, para todo $a<0$ e todo $1 \leq i \leq n$. Portanto, pela Definição 1.3 .5 , temos que $\left\|x_{i}\right\| \leq 1$, para todo $1 \leq i \leq n$, o que implica que $\|x\|:=\max \left\{\left\|x_{i}\right\|: 1 \leq i \leq n\right\} \leq 1$, ou seja, $x \in B_{1}^{\prime}(0)$.

Finalmente, provaremos que a afimação 3 . é verdadeira. 


$$
\text { Seja } z=\left(z_{1}, \ldots, z_{n}\right):=\left(\left[\left(z_{1 \varepsilon}\right)_{\varepsilon}\right], \ldots,\left[\left(z_{n \varepsilon}\right)_{\varepsilon}\right]\right) \in \tilde{V}_{c}, \text { onde }\left(z_{\varepsilon}\right)_{\varepsilon}:=\left(\left(z_{1 \varepsilon}, \ldots, z_{n \varepsilon}\right)\right)_{\varepsilon} \in V_{c} .
$$

Então existem $K_{2} \subset \subset V$ e $\eta_{2} \in \mathbf{I}$ tais que $z_{\varepsilon} \in K_{2}$, para todo $\varepsilon \in \mathbf{I}_{\eta_{2}}$.

Consideremos $r:=\operatorname{dist}\left(K_{2},\left\{x_{0}\right\}\right)>0$. Então $\left|z_{\varepsilon}-x_{0}\right| \geq r$, para todo $\varepsilon \in \mathbf{I}_{\eta_{2}}$, e, assim, $z-\widetilde{x}_{0}$ não é associado a zero. Logo, pela Proposição $1.4 .5(1)(\mathrm{a})$, temos que $\left\|z-\widetilde{x}_{0}\right\| \geq 1$. Dessa forma, $z \in \tilde{\Omega}_{c} \backslash B_{1}\left(\tilde{x}_{0}\right)$. Portanto, $\tilde{V}_{c} \subset \tilde{\Omega}_{c} \backslash B_{1}\left(\tilde{x}_{0}\right)$.

Suponhamos $\tilde{x}_{0}:=\left(\tilde{x}_{01}, \ldots, \tilde{x}_{0 n}\right)$. Da afirmação 1 . e de $\tilde{x}_{0} \in \tilde{\Omega}_{c}$, temos que

$$
\left\|\widetilde{x}_{0}\right\| \leq 1 \quad \text { e } \quad \widetilde{\Omega}_{c} \backslash B_{1}\left(\widetilde{x}_{0}\right) \subset B_{1}^{\prime}(0) \backslash B_{1}\left(\widetilde{x}_{0}\right)
$$

Afirmamos que $B_{1}^{\prime}(0) \backslash B_{1}\left(\widetilde{x}_{0}\right) \subset S_{1}\left(\widetilde{x}_{0}\right)$. De fato, seja $y=\left(y_{1}, \ldots, y_{n}\right) \in B_{1}^{\prime}(0) \backslash B_{1}\left(\widetilde{x}_{0}\right)$. Então $\|y\| \leq 1$ e $\left\|y-\widetilde{x}_{0}\right\| \geq 1$ e, portanto, pela Proposição 1.3 .7 (1) e (4), segue que

$$
1 \leq\left\|y-\widetilde{x}_{0}\right\|=\max \left\{\left\|y_{i}-\widetilde{x}_{0 i}\right\|: 1 \leq i \leq n\right\} \leq \max \left\{\left\|y_{i}\right\|,\left\|\widetilde{x}_{0 i}\right\|: 1 \leq i \leq n\right\} \leq 1
$$

ou seja, $\left\|y-\tilde{x}_{0}\right\|=1$, o que implica que $y \in S_{1}\left(\widetilde{x}_{0}\right)$. Logo, $\widetilde{\Omega}_{c} \backslash B_{1}\left(\widetilde{x}_{0}\right) \subset S_{1}\left(\widetilde{x}_{0}\right)$.

\subsection{Uma ultramétrica para $\mathcal{G}(\Omega ; \mathbb{K})$}

Seja $\Omega$ um subconjunto aberto de $\mathbb{R}^{n}$. Nesta seção, apresentaremos a topologia cortante sobre $\mathcal{G}(\Omega ; \mathbb{K})$ desenvolvida por D. Scarpalezos mas com a abordagem feita por J. Aragona, R. Fernandez e S. O. Juriaans ([AFJ-3]).

As notações utilizadas nesta seção são as das seção 1.5, a menos de menção em contrário.

Seja $\left(\Omega_{m}\right)_{m \in N}$ uma seqüência exaustiva de abertos de $\Omega$, isto é, $\Omega=\bigcup_{m \in N} \Omega_{m}$, onde $\Omega_{m}$ é um subconjunto aberto de $\mathbb{R}^{n}$ e $\bar{\Omega}_{m} \subset \subset \Omega_{m+1}$, para todo $m \in \mathbb{N}$.

D. Scarpalezos, para cada $\left(f_{\varepsilon}\right)_{\varepsilon} \in \mathcal{E}_{M}[\Omega]$ e cada $(m, p) \in \mathbb{N}^{2}$, define $S_{m, p}\left(\left(f_{\varepsilon}\right)_{\varepsilon}\right):=\left\{r \in \mathbb{R}: \forall \alpha \in \mathbb{N}^{n} \operatorname{com}|\alpha| \leq p\right.$, tem-se $\left\|\partial^{\alpha} f_{\varepsilon}(\cdot)\right\|_{\Omega_{m}}=o\left(\varepsilon^{r}\right)$, quando $\left.\varepsilon \downarrow 0\right\}$ 
e

$$
V_{m, p}\left(\left(f_{\varepsilon}\right)_{\varepsilon}\right):= \begin{cases}\sup S_{m, p}\left(\left(f_{\varepsilon}\right)_{\varepsilon}\right) & , \text { se } S_{m, p}\left(\left(f_{\varepsilon}\right)_{\varepsilon}\right) \text { é limitado superiormente } \\ +\infty & , \text { caso contrário }\end{cases}
$$

e obteve que a função $d_{m, p}: \mathcal{G}(\Omega) \times \mathcal{G}(\Omega) \longrightarrow \mathbb{R}_{+}$, dada por

$$
d_{m, p}\left(\left[\left(f_{\varepsilon}\right)_{\varepsilon}\right],\left[\left(g_{\varepsilon}\right)_{\varepsilon}\right]\right):= \begin{cases}e^{-V_{m, p}\left(\left(f_{\varepsilon}-g_{\varepsilon}\right)_{\varepsilon}\right)} & , \text { se } V_{m, p}\left(\left(f_{\varepsilon}-g_{\varepsilon}\right)_{\varepsilon}\right) \in \mathbb{R} \\ 0 & , \text { se } V_{m, p}\left(\left(f_{\varepsilon}-g_{\varepsilon}\right)_{\varepsilon}\right)=+\infty\end{cases}
$$

é uma pseudo-ultramétrica sobre $\mathcal{G}(\Omega)$. A topologia de D. Scarpalezos para $\mathcal{G}(\Omega)$ é a topologia associada à estrutura uniforme gerada pela família $\left\{d_{m, p}\right\}_{(m, p) \in N^{2}}$ e é chamada de topologia cortante sobre $\mathcal{G}(\Omega)$.

J. Aragona, R. Fernandez e S. O. Juriaans observaram que a função $d_{m, p}$ poderia ser apresentada sem fazer referência aos representantes dos elementos de $\mathcal{G}(\Omega)$, utilizando, para isso, a relação de associação em $\overline{\mathbb{K}}$. A seguir, apresentaremos essa nova abordagem.

Lembramos que $\rho: M \times M \longrightarrow \mathbb{R}_{+}$é uma pseudo-ultramétrica sobre um conjunto $M$ se, e somente se,

- $\rho(a, a)=0, \quad \forall a \in M$

- $\rho(a, b)=\rho(b, a), \quad \forall a, b \in M$;

- $\rho(a, b) \leq \max \{\rho(a, c), \rho(c, b)\}, \quad \forall a, b, c \in M$.

Proposição 1.7.1 Sejam $\Omega$ um subconjunto aberto de $\mathbb{R}^{n},\left(\Omega_{m}\right)_{m \in N}$ uma seqüência exaustiva de abertos de $\Omega, \beta \in \mathbb{N}^{n}$ e $\left(f_{\varepsilon}\right)_{\varepsilon},\left(g_{\varepsilon}\right)_{\varepsilon} \in \mathcal{E}_{M}[\Omega]$. Tem-se que:

1. $\left(\left\|\partial^{\beta} f_{\varepsilon}(\cdot)\right\|_{\Omega_{m}}\right)_{\varepsilon} \in \mathcal{E}_{M}(\mathbb{K}), \forall m \in \mathbb{N}$;

2. $s e\left(f_{\varepsilon}-g_{\varepsilon}\right)_{\varepsilon} \in \mathcal{N}[\Omega]$, então $\left(\left\|\partial^{\beta} f_{\varepsilon}(\cdot)\right\|_{\Omega_{m}}-\left\|\partial^{\beta} g_{\varepsilon}(\cdot)\right\|_{\Omega_{m}}\right)_{\varepsilon} \in \mathcal{N}(\mathbb{K}), \forall m \in \mathbb{N}$. 
Demonstração: Seja $m \in \mathbb{N}$. De $\bar{\Omega}_{m} \subset \subset \Omega$ e $\left(f_{\varepsilon}\right)_{\varepsilon} \in \mathcal{E}_{M}[\Omega]$ temos, pela Proposição 1.1.4 e Proposição 1.5.5 (1), que $\left(\left\|\partial^{\beta} f_{\varepsilon}(\cdot)\right\|_{\bar{\Omega}_{m}}\right)_{\varepsilon} \in \mathcal{E}_{M}(\mathbb{K})$. Assim, 1. é verdadeira, pois $\left\|\partial^{\beta} f_{\varepsilon}(\cdot)\right\| \Omega_{\Omega_{m}} \leq\left\|\partial^{\beta} f_{\varepsilon}(\cdot)\right\|_{\bar{\Omega}_{m}}$, para todo $\varepsilon \in \mathbf{I}$.

Para provarmos 2., notemos que, para todo $x \in \Omega_{m}$ e todo $\varepsilon \in \mathbf{I}$, temos que

$$
\begin{aligned}
& \left|\partial^{\beta} f_{\varepsilon}(x)\right| \leq\left|\partial^{\beta}\left(f_{\varepsilon}-g_{\varepsilon}\right)(x)\right|+\left|\partial^{\beta} g_{\varepsilon}(x)\right| \leq\left.\left\|\partial^{\beta}\left(f_{\varepsilon}-g_{\varepsilon}\right)(\cdot)\right\|\right|_{\Omega_{m}}+\left.\left\|\partial^{\beta} g_{\varepsilon}(\cdot)\right\|\right|_{\Omega_{m}} \\
& \left|\partial^{\beta} g_{\varepsilon}(x)\right| \leq\left|\partial^{\beta}\left(g_{\varepsilon}-f_{\varepsilon}\right)(x)\right|+\left|\partial^{\beta} f_{\varepsilon}(x)\right| \leq\left\|\partial^{\beta}\left(f_{\varepsilon}-g_{\varepsilon}\right)(\cdot)\right\|_{\Omega_{m}}+\left\|\partial^{\beta} f_{\varepsilon}(\cdot)\right\| \|_{\Omega_{m}} .
\end{aligned}
$$

Logo, para todo $\varepsilon \in \mathbf{I}$, temos que

$$
\begin{aligned}
& \left\|\partial^{\beta} f_{\varepsilon}(\cdot)\right\|_{\Omega_{m}} \leq\left\|\partial^{\beta}\left(f_{\varepsilon}-g_{\varepsilon}\right)(\cdot)\right\|_{\Omega_{m}}+\left\|\partial^{\beta} g_{\varepsilon}(\cdot)\right\|_{\Omega_{m}} \\
& \left\|\partial^{\beta} g_{\varepsilon}(\cdot)\right\|_{\Omega_{m}} \leq\left\|\partial^{\beta}\left(f_{\varepsilon}-g_{\varepsilon}\right)(\cdot)\right\|_{\Omega_{m}}+\left\|\partial^{\beta} f_{\varepsilon}(\cdot)\right\| \|_{\Omega_{m}}
\end{aligned}
$$

ou seja,

$$
\left|\left\|\partial^{\beta} f_{\varepsilon}(\cdot)\right\|\right|_{\Omega_{m}}-\left.\left\|\partial^{\beta} g_{\varepsilon}(\cdot)\right\|\right|_{\Omega_{m}} \mid \leq\left\|\partial^{\beta}\left(f_{\varepsilon}-g_{\varepsilon}\right)(\cdot)\right\|_{\Omega_{m}} \leq\left\|\partial^{\beta}\left(f_{\varepsilon}-g_{\varepsilon}\right)(\cdot)\right\|_{\bar{\Omega}_{m}} .
$$

De $\bar{\Omega}_{m} \subset \subset \Omega$ e $\left(f_{\varepsilon}-g_{\varepsilon}\right)_{\varepsilon} \in \mathcal{N}[\Omega]$ obtemos, pela Proposição 1.1.5 e Proposição 1.5.5 (2), que $\left(\left\|\partial^{\beta}\left(f_{\varepsilon}-g_{\varepsilon}\right)(\cdot)\right\|_{\bar{\Omega}_{m}}\right)_{\varepsilon} \in \mathcal{N}(\mathbb{K})$ e, assim, de (1.17), concluímos que afirmação 2. é verdadeira.

Em virtude da Proposição anterior, faz sentido a definição a seguir.

Definição 1.7.2 Sejam $\Omega$ um subconjunto aberto de $\mathbb{R}^{n},\left(\Omega_{m}\right)_{m \in N}$ uma seqüência exaustiva de abertos de $\Omega$ e $f \in \mathcal{G}(\Omega)$. Define-se, para cada $(\beta, m) \in \mathbb{N}^{n} \times \mathbb{N}$,

$$
|f|_{\beta, m}:=\left[\left(\left\|\partial^{\beta} f_{\varepsilon}(\cdot)\right\|_{\Omega_{m}}\right)_{\varepsilon}\right] \in \overline{\mathbb{K}}
$$

onde $\left(f_{\varepsilon}\right)_{\varepsilon}$ é um representante qualquer de $f$.

O conjunto $S_{m, p}\left(\left(f_{\varepsilon}\right)_{\varepsilon}\right)$ definido por D. Scarpalezos será substituído por: 
Definição 1.7.3 Sejam $\Omega$ um subconjunto aberto de $\mathbb{R}^{n},\left(\Omega_{m}\right)_{m \in \mathbb{N}}$ uma seqüência exaustiva de abertos de $\Omega$ e $f \in \mathcal{G}(\Omega)$. Define-se, para cada $(m, p) \in \mathbb{N}^{2}$,

$$
A_{m, p}(f):=\left\{r \in \mathbb{R}: \forall \beta \in \mathbb{N}^{n} \text { com }|\beta| \leq p, \text { tem-se } \alpha_{-r}|f|_{\beta, m} \approx 0\right\}
$$

Convém observarmos que, se $f \in \mathcal{G}(\Omega)$, então, da definição de $\mathcal{E}_{M}[\Omega]$, temos que $A_{m, p}(f) \neq \varnothing$, para todo $(m, p) \in \mathbb{N}^{2}$. Notamos que, se $\left(f_{\varepsilon}\right)_{\varepsilon}$ é um representante de $f$, $\beta \in \mathbb{N}^{n},(m, p) \in \mathbb{N}^{2}$ e $r \in \mathbb{R}$, então

$$
\left\|\partial^{\beta} f_{\varepsilon}(\cdot)\right\|_{\Omega_{m}}=o\left(\varepsilon^{r}\right) \text {, quando } \varepsilon \downarrow 0 \Longleftrightarrow \lim _{\varepsilon \downarrow 0} \frac{\left\|\partial^{\beta} f_{\varepsilon}(\cdot)\right\|_{\Omega_{m}}}{\varepsilon^{r}}=0 \Longleftrightarrow \alpha_{-r}|f|_{\beta, m} \approx 0 .
$$

Logo, para todo $\left(f_{\varepsilon}\right)_{\varepsilon}$ representante de $f$, temos que $S_{m, p}\left(\left(f_{\varepsilon}\right)_{\varepsilon}\right)=A_{m, p}(f)$ e, se $A_{m, p}(f)$ é limitado superiormente, então $V_{m, p}\left(\left(f_{\varepsilon}\right)_{\varepsilon}\right):=\sup A_{m, p}(f)$.

Definição 1.7.4 Sejam $\Omega$ um subconjunto aberto de $\mathbb{R}^{n},\left(\Omega_{m}\right)_{m \in \mathbb{N}}$ uma seqüência exaustiva de abertos de $\Omega$ e $f \in \mathcal{G}(\Omega)$. Define-se, para cada $(m, p) \in \mathbb{N}^{2}$,

$$
\mathcal{V}_{m, p}(f):=\left\{\begin{array}{ll}
\sup A_{m, p}(f) & \text {, se } A_{m, p}(f) \text { é limitado superiormente } \\
+\infty & , \text { caso contrário }
\end{array} .\right.
$$

Notamos que $A_{m, p}(\cdot)$ tem as seguintes propriedades:

Proposição 1.7.5 Sejam $\Omega$ um subconjunto aberto de $\mathbb{R}^{n},\left(\Omega_{m}\right)_{m \in N}$ uma seqüência exaustiva de abertos de $\Omega, f \in \mathcal{G}(\Omega),(m, p) \in \mathbb{N}^{2}$. As seguintes afirmações são válidas:

1. se $a, b \in \mathbb{R}, a \in A_{m, p}(f)$ e $b<a$, ent $\tilde{a} o b \in A_{m, p}(f)$;

2. $A_{m, p}(f)=\mathbb{R}$, ou existe $r \in \mathbb{R}$ tal que $\left.A_{m, p}(f)=\right]-\infty, r\left[\right.$ ou $\left.\left.A_{m, p}(f)=\right]-\infty, r\right]$.

Demonstração: A afirmação 1. segue da Proposição 1.1 .12 (5) e a afirmação 2. segue de $1 .$.

Apresentamos, a seguir, algumas propriedades de $\mathcal{V}_{m, p}(\cdot)$. 
Proposição 1.7.6 Sejam $\Omega$ um subconjunto aberto de $\mathbb{R}^{n},\left(\Omega_{m}\right)_{m \in N}$ uma seqüência exaustiva de abertos de $\Omega, f \in \mathcal{G}(\Omega),(m, p) \in \mathbb{N}^{2}$ e $c \in \mathbb{R}$. Tem-se que:

1. $\mathcal{V}_{m, p}(f)=+\infty$ se, e somente se, $A_{m, p}(f)=\mathbb{R}$;

2. $f=0$ se, e somente se, $\mathcal{V}_{k, 0}(f)=+\infty$, para todo $k \in \mathbb{N}$;

3. se $\mathcal{V}_{m, p}(f)=+\infty$, ou se $c<\mathcal{V}_{m, p}(f)<+\infty$, então $c \in A_{m, p}(f)$.

Demonstração: A primeira afirmação decorre da Proposição 1.7.5 (2). A terceira, no caso $\mathcal{V}_{m, p}(f)=+\infty$, segue de 1 . e, no caso de $\mathcal{V}_{m, p}(f) \in \mathbb{R}$, é conseqüência da Proposição 1.7.5 (1) e da definição de sup. Para provarmos a segunda afirmação, basta, para $\left(f_{\varepsilon}\right)_{\varepsilon}$ representante de $f$, observarmos que

$$
\begin{aligned}
f=0 & \Longleftrightarrow \lim _{\varepsilon \downarrow 0} \frac{\|\left. f_{\varepsilon}(\cdot)\right|_{K}}{\varepsilon^{r}}=0, \forall K \subset \subset \Omega, \forall r \in \mathbb{R} \\
& \Longleftrightarrow \lim _{\varepsilon \downarrow 0} \frac{\|\left. f_{\varepsilon}(\cdot)\right|_{\Omega_{k}}}{\varepsilon^{r}}=0, \forall k \in \mathbb{N}, \forall r \in \mathbb{R} \\
& \Longleftrightarrow \alpha_{-r}|f|_{0, k} \approx 0, \forall k \in \mathbb{N}, \forall r \in \mathbb{R} \\
& \Longleftrightarrow A_{k, 0}(f)=\mathbb{R}, \forall k \in \mathbb{N} \\
& \Longleftrightarrow \mathcal{V}_{k, 0}(f)=+\infty, \forall k \in \mathbb{N},
\end{aligned}
$$

onde a primeira equivalência segue do Teorema 1.5.6 e a última da afirmação $1 .$.

Definição 1.7.7 Sejam $\Omega$ um subconjunto aberto de $\mathbb{R}^{n}$ e $\left(\Omega_{m}\right)_{m \in N}$ uma seqüência exaustiva de abertos de $\Omega$. Para cada $(m, p) \in \mathbb{N}^{2}$, define-se $D_{m, p}: \mathcal{G}(\Omega) \times \mathcal{G}(\Omega) \longrightarrow \mathbb{R}_{+}$ por

$$
D_{m, p}(f, g):= \begin{cases}e^{-\mathcal{V}_{m, p}(f-g)} & , \text { se } \mathcal{V}_{m, p}(f-g) \in \mathbb{R} \\ 0 & , \text { se } \mathcal{V}_{m, p}(f-g)=+\infty\end{cases}
$$

Notamos que a função $D_{m, p}$ coincide com a função $d_{m, p}$ definida por D. Scarpalezos, cujas propriedades foram provadas, detalhadamente, por A. A. Silva ([Sil-A]). Por essa razão, nós omitiremos as provas das propriedades de $D_{m, p}$ que serão enunciadas a seguir. 
Se $\Omega$ é um subconjunto aberto de $\mathbb{R}^{n},(m, p) \in \mathbb{N}^{2}$ ef $, g, h \in \mathcal{G}(\Omega)$, então

- $D_{m, p}$ é uma pseudo-ultramétrica sobre $\mathcal{G}(\Omega)$;

- $D_{m, p}(f, g) \leq \max \left\{D_{m, p}(f, 0), D_{m, p}(g, 0)\right\}$;

- $D_{m, p}(f g, 0) \leq D_{m, p}(f, 0) D_{m, p}(g, 0)$;

- $D_{m, p}(x f, 0) \leq\|x\| D_{m, p}(f, 0), \forall x \in \overline{\mathbb{K}}$;

- $D_{m, p}\left(k \alpha_{r} f, 0\right)=e^{-r} D_{m, p}(f, 0), \forall k \in \mathbb{K ^ { * }}, \forall r \in \mathbb{R}$;

- $D_{m, p}\left(\partial^{\beta} f, 0\right) \leq D_{m, p+|\beta|}(f, 0), \forall \beta \in \mathbb{N}^{n}$;

- $D_{m, p}$ é invariante por translaçôes, isto é, $D_{m, p}(f, g)=D_{m, p}(f+h, g+h)$, em particular,

- $D_{m, p}(f, g)=D_{m, p}(f-g, 0)$.

Definição 1.7.8 A estrutura uniforme sobre $\mathcal{G}(\Omega)$ gerada pela família $\left\{D_{m, p}\right\}_{(m, p) \in N^{2}}$ de pseudo-ultramétricas sobre $\mathcal{G}(\Omega)$ é chamada de estrutura uniforme cortante de $\mathcal{G}(\Omega)$ e é denotada por $\mathcal{U}_{\Omega}$.

Definição 1.7.9 A topologia sobre $\mathcal{G}(\Omega)$ gerada por $\mathcal{U}_{\Omega}$ é chamada de topologia cortante de $\mathcal{G}(\Omega)$ e é denotada por $\mathscr{T}_{\Omega}$.

Maiores detalhes sobre estrutura uniforme e sobre a topologia gerada por uma estrutura uniforme podem ser encontrados em [Kel].

No que segue, denotamos $D_{m, m}$ (definida na Definição 1.7 .7 ), por $D_{m}$, para todo $m \in \mathbb{N}$. 
Definição 1.7.10 Sejam $\Omega$ um subconjunto aberto de $\mathbb{R}^{n}$ e $\left(\Omega_{m}\right)_{m \in N}$ uma seqüência exaustiva de abertos de $\Omega$. Define-se $D^{*}: \mathcal{G}(\Omega) \times \mathcal{G}(\Omega) \longrightarrow \mathbb{R}_{+}$por

$$
D^{*}(f, g):=\sup \left\{\frac{D_{m}(f, g)}{2^{m}\left(1+D_{m}(f, g)\right)}: m \in \mathbb{N}\right\} \text {. }
$$

Observamos que $D^{*}$ é uma ultramétrica sobre $\mathcal{G}(\Omega)$ tal que a topologia associada a $D^{*}$ coincide com a topologia cortante $\mathscr{T}_{\Omega}$ e que o espaço ultramétrico $\left(\mathcal{G}(\Omega), \mathscr{T}_{\Omega}\right)$ é completo ([Sil-A, Teorema 2.3.18] e [NPS, Teorema 1.1]).

Em seguida, munindo $\mathcal{G}(\Omega)$ com a topologia associada a $D^{*}$, finalizamos esta seção com um resultado que será útil no capítulo 4 .

Proposição 1.7.11 Sejam $\Omega$ um subconjunto aberto de $\mathbb{R}^{n},\left(\Omega_{m}\right)_{m \in N}$ uma seqüência exaustiva de abertos de $\Omega$ e $\left(f_{k}\right)_{k \in N}$ uma seqüência de elementos de $\mathcal{G}(\Omega)$. Se $\lim _{k \rightarrow \infty} f_{k}=0$, então, para todo $m \in \mathbb{N}$ e para todo $q \in \mathbb{N}$,

$$
\text { existe } N=N_{m, q} \in \mathbb{N} \text { tal que } \alpha_{-q}\left|f_{k}\right|_{\beta, m} \approx 0, \forall k \geq N, \forall \beta \in \mathbb{N}^{n} \text { com }|\beta| \leq m \text {. }
$$

Demonstração: Notemos que, de

$$
D^{*}\left(f_{k}, 0\right)=\sup \left\{\frac{D_{s}\left(f_{k}, 0\right)}{2^{s}\left(1+D_{s}\left(f_{k}, 0\right)\right)}: s \in \mathbb{N}\right\}, \forall k \in \mathbb{N},
$$

segue que

$$
\frac{D_{s}\left(f_{k}, 0\right)}{2^{s}\left(1+D_{s}\left(f_{k}, 0\right)\right)} \leq D^{*}\left(f_{k}, 0\right), \forall s \in \mathbb{N}, \forall k \in \mathbb{N} .
$$

Sejam $m \in \mathbb{I}$ e $q \in \mathbb{I}$. De $\lim _{k \rightarrow \infty} f_{k}=0$, existe $N \in \mathbb{N}$ tal que

$$
0 \leq D^{*}\left(f_{k}, 0\right)<\frac{e^{-q}}{2^{m}\left(1+e^{-q}\right)}, \forall k \geq N,
$$

e, assim, de (1.18), temos que

$$
\frac{D_{m}\left(f_{k}, 0\right)}{1+D_{m}\left(f_{k}, 0\right)}<\frac{e^{-q}}{1+e^{-q}}, \forall k \geq N
$$


Sejam $k \geq N$ e $\beta \in \mathbb{N}^{n} \operatorname{com}|\beta| \leq m$. Se $\mathcal{V}_{m, m}\left(f_{k}\right)=+\infty$, então, pela Proposição 1.7.6 (1), temos que $A_{m, m}(f)=\mathbb{R}$ e disto resulta $\alpha_{-q}\left|f_{k}\right|_{\beta, m} \approx 0$. Se $\mathcal{V}_{m, m}\left(f_{k}\right) \in \mathbb{R}$, então, de (1.19), obtemos que

$$
\frac{e^{-\mathcal{V}_{m, m}\left(f_{k}\right)}}{1+e^{-\mathcal{V}_{m, m}\left(f_{k}\right)}}<\frac{e^{-q}}{1+e^{-q}}
$$

e, desse modo, usando que a função $g(r)=\frac{r}{1+r}$, onde $r \in \mathbb{R}_{+}$, é não-decrescente, temos que

$$
e^{-\mathcal{V}_{m, m}\left(f_{k}\right)}<e^{-q}
$$

ou seja, $\mathcal{V}_{m, m}\left(f_{k}\right)>q$. Portanto, pela Proposição 1.7.6 (3), temos que $q \in A_{m, m}(f)$, de onde obtemos $\alpha_{-q}\left|f_{k}\right|_{\beta, m} \approx 0$.

Finalizamos este capítulo com a próxima seção. Nela apresentamos, a partir da relação de ordem parcial sobre $\overline{\mathbb{R}}$ (Definição 1.2.2), uma topologia sobre $\overline{\mathbb{K}}$ e uma sobre $\mathcal{G}(\Omega)$. Essas topologias coincidem com a topologia cortante sobre $\bar{K}$ e com a topologia cortante sobre $\mathcal{G}(\Omega)$, respectivamente.

\subsection{Topologias metrizáveis sobre $\overline{\mathbb{K}}$ e sobre $\mathcal{G}(\Omega ; \mathbb{K})$}

J. Aragona, R. Fernandez e S. O. Juriaans ([AFJ-2] e [AFJ-3]) provaram que, com a relação de ordem parcial sobre $\overline{\mathbb{R}}$, é possível definir uma topologia sobre $\overline{\mathbb{K}}$ e uma sobre $\mathcal{G}(\Omega)$ que coincidem com as topologias desenvolvidas por D. Scarpalezos (apresentadas nas seções 1.3 e 1.7). Por essas topologias serem interessantes e poderem ser úteis em outros trabalhos, nós iremos apresentá-las nesta seção. No entanto, como elas não serão utilizadas ao longo desta Dissertação, nós omitiremos as demonstrações (as quais podem ser encontrados em [AFJ-2] e [AFJ-3]) dos resultados aqui apresentados.

Lembramos que, se $X$ é um conjunto e $\mathscr{B}$ é uma família de subconjuntos de $X$ tal que

1. $\varnothing \notin \mathscr{B}$ e $\mathscr{B} \neq \varnothing$; 
2. se $B_{1}, B_{2} \in \mathscr{B}$, então existe $B_{3} \in \mathscr{B}$ tal que $B_{3} \in B_{1} \cap B_{2}$;

então $\mathscr{B}$ é uma base de filtro em $X$.

Recordamos, também, que uma estrutura de anel e uma topologia sobre um conjunto $A$ são compatíveis se as aplicações $(x, y) \in A \times A \longmapsto x+y \in A, x \in A \longmapsto-x \in A \mathrm{e}$ $(x, y) \in A \times A \longmapsto x y \in A$ são contínuas.

O resultado de J. Aragona, R. Fernandez e S. O. Juriaans ([AFJ-2] e [AFJ-3]), é o seguinte:

Sejam $\Omega$ um subconjunto aberto de $\mathbb{R}^{n}$ e $\left(\Omega_{m}\right)_{m \in \mathbb{N}}$ uma seqüencia exaustiva de abertos de $\Omega$. Para cada $x_{0} \in \overline{\mathbb{K}}, f_{0} \in \mathcal{G}(\Omega), \beta \in \mathbb{N}^{n}, m \in \mathbb{N}$ e $r \in \mathbb{R}$, sejam

$$
\begin{gathered}
V_{r}\left(x_{0}\right):=\left\{x \in \overline{\mathbb{K}}:\left|x-x_{0}\right| \leq \alpha_{r}\right\} ; \\
W_{m, r}^{\beta}\left(f_{0}\right):=\left\{f \in \mathcal{G}(\Omega):|| f-\left.f_{0}\right|_{\sigma, m} \mid \leq \alpha_{r}, \forall \sigma \leq \beta\right\} ;
\end{gathered}
$$

onde $\alpha_{r}$ é a classe em $\overline{\mathbb{R}}$ de $\left(\varepsilon^{r}\right)_{\varepsilon} e\left|f-f_{0}\right|_{\sigma, m}$ é como na Definição 1.7.2. Se

$$
\mathcal{B}:=\left\{V_{r}(0): r \in \mathbb{R}\right\} \quad \text { e } \quad \mathcal{B}_{\Omega}:=\left\{W_{m, r}^{\beta}(0): \beta \in \mathbb{N}^{n}, m \in \mathbb{N}, r \in \mathbb{R}\right\}
$$

então

- $\mathcal{B}$ e $\mathcal{B}_{\Omega}$ são bases de filtro sobre $\overline{\mathbb{K}}$ e $\mathcal{G}(\Omega)$, respectivamente;

- $\mathcal{B}$ determina uma topologia metrizável $\Im$ sobre $\overline{\mathbb{K}}$ compatível com a estrutura de anel de $\overline{\mathbb{K}}$ e $\mathcal{B}_{\Omega}$ determina uma topologia metrizável $\Im_{\Omega}$ sobre $\mathcal{G}(\Omega)$ compativel com a estrutura de anel de $\mathcal{G}(\Omega)$;

- $\mathcal{B}$ é um sistema fundamental de vizinhanças de zero em $(\overline{\mathbb{K}}, \Im)$ e $\mathcal{B}_{\Omega}$ é um sistema fundamental de vizinhanças de zero em $\left(\mathcal{G}(\Omega), \Im_{\Omega}\right)$;

- $\Im$ coincide com a topologia cortante $\mathscr{T}_{c}$ e $\Im_{\Omega}$ coincide com a topologia cortante $\mathscr{T}_{\Omega}$, onde $\mathscr{T}_{c}$ e $\mathscr{T}_{\Omega}$ foram definidas nas seções 1.3 e 1.7, respectivamente. 


\section{Capítulo 2}

\section{Limite e diferenciabilidade}

Este capítulo tem como objetivo ser uma introdução ao cálculo diferencial para funções de uma e de várias variáveis generalizadas - introduzido e desenvolvido por J. Aragona, R. Fernandez e S. O. Juriaans em [AFJ-1]. O conceito de derivada, para funções definidas em subconjuntos abertos de $\overline{\mathbb{K}}$ e a valores em $\overline{\mathbb{K}}$, e algumas propriedades das funções deriváveis são apresentados na seção 2.2. A seguir, introduzimos a noção de diferenciabilidade para funções de várias variáveis reais generalizadas. Esse conceito será estendido, na seção 2.4, para aplicações de variáveis reais generalizadas. Antes, porém, na seção 2.1 recordamos a noção de limite e apresentamos alguns resultados sobre limites de funções e sobre continuidade.

A partir daqui, $\bar{K}$ será munido com a ultramétrica definida por D. Scarpalezos (Definição 1.3.5) e $\overline{\mathbb{K}}^{n}$ pela ultramétrica da Definição 1.3.10.

\subsection{Limite}

Nesta seção, apresentamos alguns resultados que nos serão úteis neste trabalho e que não são verdadeiros, de modo geral, em espaços métricos. Primeiramente, notemos que: 
Se $U$ é um subconjunto aberto de $\overline{\mathbb{K}}^{n}$ e $x_{0}=\left(x_{01}, \ldots, x_{0 n}\right) \in U$, então $x_{0}$ é ponto de acumulação de $U$.

De fațo, seja $r \in \mathbb{R}$. Consideremos $s \in \mathbb{R}$ tal que $B_{s}\left(x_{0}\right) \subset U, p \in \mathbb{R}$ tal que $e^{-p}<$ $\min \{r, s\}$ e $x:=\left(x_{01}+\alpha_{p}, \ldots, x_{0 n}+\alpha_{p}\right)$. Pela Definição 1.3.10 e pela Proposição 1.3.7 (8), temos que $\left\|x-x_{0}\right\|_{n}=\left\|\alpha_{p}\right\|=e^{-p}$. Portanto, $x \in\left(B_{r}\left(x_{0}\right) \cap U\right) \backslash\left\{x_{0}\right\}$.

Portanto, se $U$ é um subconjunto aberto de $\overline{\mathbb{K}}^{n}, x_{0} \in U$ e $f: U \longrightarrow \overline{\mathbb{K}}^{m}$, então faz sentido a expressão $\lim _{x \rightarrow x_{0}} f(x)$. Além disso, se $L \in \overline{\mathbb{K}}^{m}$, lembramos que

$$
\lim _{x \rightarrow x_{0}} f(x)=L \text { se, e somente se, }
$$

$\forall \sigma>0, \exists \delta>0$ tal que se $x \in U$ e $0<\left\|x-x_{0}\right\|_{n}<\delta$, então $\|f(x)-L\|_{m}<\sigma$.

Os próximos resultados sobre limites são análogos aos do contexto clássico para funções a valores reais.

Proposição 2.1.1 Sejam $U$ um subconjunto aberto de $\overline{\mathbb{K}}^{n}, f: U \longrightarrow \overline{\mathbb{K}}, x_{0} \in U \boldsymbol{e}$ $L \in \overline{\mathbb{K}}$. São verdadeiras as seguintes afirmações:

1. se $\lim _{x \rightarrow x_{0}} f(x)=L$, então existe $V$ uma vizinhança aberta de $x_{0}$ com $V \subset U$ e tal que $\|f(x)\| \leq 1+\|L\|$, para todo $x \in V \backslash\left\{x_{0}\right\}$, e, se $L \neq 0$, então $V$ pode ser determinada de modo que $\frac{\|L\|}{2} \leq\|f(x)\| \leq \frac{3\|L\|}{2}$, para todo $x \in V \backslash\left\{x_{0}\right\}$;

2. se $\lim _{x \rightarrow x_{0}} f(x)=L$, então $\lim _{x \rightarrow x_{0}}\|f(x)\|=\|L\|$.

Demonstração: Para a afirmação 1. basta observarmos que

$$
\begin{gathered}
\|f(x)\|=\|(f(x)-L)+L\| \leq \max \{\|f(x)-L\|,\|L\|\} \leq\|f(x)-L\|+\|L\| ; \\
\|L\|=\|(L-f(x))+f(x)\| \leq \max \{\|L-f(x)\|,\|f(x)\|\} \leq\|L-f(x)\|+\|f(x)\|,
\end{gathered}
$$


onde a primeira desigualdade de cada uma dessas expressões decorre da Proposição 1.3.7 (1), e usarmos a definição de limite. Para provarmos a afirmação 2. é suficiente notarmos que $|\|f(x)\|-\|L\|| \leq\|f(x)-L\|$ (Proposição 1.3 .7 (11)).

Teorema 2.1.2 Sejam $U$ um subconjunto aberto de $\overline{\mathbb{I}}^{n}, x_{0} \in U, \lambda \in \overline{\mathbb{K}}$ ef, $g: U \longrightarrow \overline{\mathbb{I}}$ tais que existem $\lim _{x \rightarrow x_{0}} f(x)=: L_{1} e \lim _{x \rightarrow x_{0}} g(x)=: L_{2}$. São verdadeiras as seguintes asserções:

1. $\lim _{x \rightarrow x_{0}}(\lambda f(x))=\lambda L_{1}, \quad \lim _{x \rightarrow x_{0}}(f(x)+g(x))=L_{1}+L_{2}, \quad e \quad \lim _{x \rightarrow x_{0}}(f(x) g(x))=L_{1} L_{2}$;

2. se $f(U) \subset \operatorname{Inv}(\overline{\mathbb{K}}), L_{1} \in \operatorname{Inv}(\overline{\mathbb{K}})$ e $\frac{1}{f}: U \longrightarrow \overline{\mathbb{K}}$ é dada por $\left(\frac{1}{f}\right)(x):=\frac{1}{f(x)}$, isto é, $\left(\frac{1}{f}\right)(x)=(f(x))^{-1}$, ent $\tilde{a} o \lim _{x \rightarrow x_{0}} \frac{1}{f(x)}=\frac{1}{L_{1}}$.

Demonstração: Observemos, pela Proposição 1.3.7 (1), (2) e (4), que

$$
\begin{gathered}
\left\|\lambda f(x)-\lambda L_{1}\right\| \leq\|\lambda\|\left\|f(x)-L_{1}\right\| ; \\
\left\|(f(x)+g(x))-\left(L_{1}+L_{2}\right)\right\| \leq \max \left\{\left\|f(x)-L_{1}\right\|,\left\|g(x)-L_{2}\right\|\right\} ; \\
\left\|f(x) g(x)-L_{1} L_{2}\right\| \leq \max \left\{\left\|f(x)-L_{1}\right\|\left\|L_{2}\right\|,\|f(x)\|\left\|g(x)-L_{2}\right\|\right\} .
\end{gathered}
$$

Logo, pela Proposição 2.1.1 (2) e pela definição de limite, obtemos 1..

Notemos, pela Proposição 1.3.7 (2) e (4), que

$$
\left\|(f(x))^{-1}-\left(L_{1}\right)^{-1}\right\|=\left\|\frac{L_{1}-f(x)}{f(x) L_{1}}\right\| \leq\left\|\frac{1}{f(x)}\right\|\left\|\frac{1}{L_{1}}\right\|\left\|f(x)-L_{1}\right\| .
$$

Consideremos $s<\left\|\frac{1}{L_{1}}\right\|^{-1}$. Desse modo, se $f(x) \in B_{s}\left(L_{1}\right)$, então, pela Proposição

1.4.7 (3), temos que

$$
\left\|\frac{1}{f(x)}\right\| \leq\left\|\frac{1}{L_{1}}\right\|
$$

Portanto, por (2.1) e pela definição de limite, concluímos a afirmação 2. . 
Em relação à continuidade tem-se, por $\overline{\mathbb{K}}^{n}$ ser espaço métrico, que:

Sejam $U$ e V subconjuntos abertos de $\overline{\mathbb{K}}^{m}$ e de $\overline{\mathbb{K}}^{n}$, respectivamente, $f: U \longrightarrow \overline{\mathbb{K}}^{n}$ e $g: V \longrightarrow \overline{\mathbb{K}}^{p}, \operatorname{com} f(U) \subset V$. Se $f$ é contínua em $x_{0} \in U$ e $g$ é contínua em $f\left(x_{0}\right)$, então $g \circ f$ é contínua em $x_{0}$.

Além disso, temos o seguinte resultado:

Teorema 2.1.3 Sejam $U$ um subconjunto aberto de $\overline{\mathbb{K}}, x_{0} \in U, \lambda \in \overline{\mathbb{K}}$ e $f, g: U \longrightarrow \bar{K}$. Se $f$ e $g$ são contínuas em $x_{0}$, então

1. $\lambda f, f+g$ e $f \cdot g$ são continuas em $x_{0}$;

2. se $f(U) \subset \operatorname{Inv}(\bar{K})$, então a função $\frac{1}{f}: U \longrightarrow \overline{\mathbb{K}}$ dada por $\left(\frac{1}{f}\right)(x):=\frac{1}{f(x)}$, isto é, $\left(\frac{1}{f}\right)(x)=(f(x))^{-1}$, é contínua em $x_{0}$.

Demonstração: Da continuidade de $f$ e $g$ em $x_{0}$, temos que

$$
\lim _{x \rightarrow x_{0}} f(x)=f\left(x_{0}\right) \quad \text { e } \quad \lim _{x \rightarrow x_{0}} g(x)=g\left(x_{0}\right) .
$$

Logo, pelo Teorema 2.1.2, obtemos que

$$
\begin{aligned}
& \lim _{x \rightarrow x_{0}}(\lambda f(x))=\lambda f\left(x_{0}\right) ; \quad \lim _{x \rightarrow x_{0}}(f(x)+g(x))=f\left(x_{0}\right)+g\left(x_{0}\right) ; \\
& \lim _{x \rightarrow x_{0}}(f(x) g(x))=f\left(x_{0}\right) g\left(x_{0}\right) ; \quad \lim _{x \rightarrow x_{0}} \frac{1}{f(x)}=\frac{1}{f\left(x_{0}\right)} ;
\end{aligned}
$$

o que prova 1 . e 2 ..

Finalizamos esta seção notando que, se $U$ é um subconjunto aberto de $\overline{\mathbb{R}}^{n}$ e $f: U \longrightarrow \overline{\mathbb{R}}^{m}$, então existem $f_{1}, \ldots, f_{m}: U \longrightarrow \overline{\mathbb{R}}$ tais que $f(x)=\left(f_{1}(x), \ldots, f_{m}(x)\right)$, para todo $x \in U$, o que nos leva a escrever $f=\left(f_{1}, \ldots, f_{m}\right)$. Além disso, se $b=\left(b_{1}, \ldots, b_{m}\right) \in \overline{\mathbb{R}}^{m}$, então

$$
\lim _{x \rightarrow x_{0}} f(x)=b \Longleftrightarrow \lim _{x \rightarrow x_{0}} f_{i}(x)=b_{i}, \forall 1 \leq i \leq m
$$




\subsection{Derivada}

Nesta seção encontra-se um conceito de derivada para funções definidas em um subconjunto aberto de $\overline{\mathbb{K}}$ e a valores em $\overline{\mathbb{K}}$. Esse conceito preserva as regras de derivação e a regra da cadeia que conhecemos no contexto clássico. No entanto, o Teorema do Valor Médio, neste contexto, é falso em geral (Observação 2.2 .5 (b)).

Iniciamos a seção com um Lema que será importante para termos, no máximo, um único elemento de $\overline{\mathbb{K}}$ satisfazendo o limite da Definição 2.2.2.

Lema 2.2.1 Sejam $U$ um subconjunto aberto de $\overline{\mathbb{K}}, f: U \longrightarrow \overline{\mathbb{K}}$ e $x_{0} \in U$. Então existe no máximo um $z_{0} \in U$ tal que

$$
\lim _{x \rightarrow x_{0}} \frac{f(x)-f\left(x_{0}\right)-z_{0}\left(x-x_{0}\right)}{\alpha_{-\ln }\left\|x-x_{0}\right\|}=0 .
$$

Demonstração: Sejam $z_{0}, z_{1} \in \bar{K}$ tais que

$$
\lim _{x \rightarrow x_{0}} \frac{f(x)-f\left(x_{0}\right)-z_{0}\left(x-x_{0}\right)}{\alpha_{-} \ln \left\|x-x_{0}\right\|}=0 \quad \text { e } \quad \lim _{x \rightarrow x_{0}} \frac{f(x)-f\left(x_{0}\right)-z_{1}\left(x-x_{0}\right)}{\alpha_{-\ln \left\|x-x_{0}\right\|}}=0 .
$$

Então, pelo Teorema 2.1.2 (1), obtemos que

$$
\lim _{x \rightarrow x_{0}} \frac{\left(z_{0}-z_{1}\right)\left(x-x_{0}\right)}{\alpha_{-} \ln \left\|x-x_{0}\right\|}=0 .
$$

Consideremos, para cada $n \in \mathbb{N}, x_{n}:=x_{0}+\alpha_{n}$. Logo, pela Proposição 1.3.7 (8), $\left\|x_{n}-x_{0}\right\|=\left\|\alpha_{n}\right\|=e^{-n}$, para todo $n \in \mathbb{N}$. Portanto, $\lim _{n \rightarrow \infty} x_{n}=x_{0}$ e, de (2.3), temos que

$$
0=\lim _{n \rightarrow \infty} \frac{\left(z_{0}-z_{1}\right)\left(x_{n}-x_{0}\right)}{\alpha_{-\ln \left\|x_{n}-x_{0}\right\|}}=\lim _{n \rightarrow \infty} \frac{\left(z_{0}-z_{1}\right) \alpha_{n}}{\alpha_{n}}=z_{0}-z_{1},
$$

ou seja, $z_{0}=z_{1}$.

Em razão do Lema anterior, faz sentido a seguinte definição: 
Definição 2.2.2 Sejam $U$ um subconjunto aberto de $\overline{\mathbb{K}}, f: U \longrightarrow \overline{\mathbb{K}}$ e $x_{0} \in U$. Diz-se que $f$ é derivável em $x_{0}$ se existe $z_{0} \in \overline{\mathbb{K}}$ tal que

$$
\lim _{x \rightarrow x_{0}} \frac{f(x)-f\left(x_{0}\right)-z_{0}\left(x-x_{0}\right)}{\alpha_{-\ln \left\|x-x_{0}\right\|}}=0 .
$$

Nesse caso, $z_{0}$ é chamada a derivada de $f$ em $x_{0}$ e denota-se $D(f)\left(x_{0}\right):=z_{0}$ ou $f^{\prime}\left(x_{0}\right):=z_{0}$. Diz-se que $f$ é derivável em $U$, se $f$ é derivável em cada ponto de $U$.

No artigo [AFJ-1] há uma explicação para a escolha do limite que aparece na Definição 2.2.2. Os autores observam que, de $\lim _{x \rightarrow x_{0}} \alpha_{-} \ln \left\|x-x_{0}\right\|=0$ (Proposição 1.3 .7 (8)), a definição dada é similar à do contexto clássico substituindo o número real invertível $x-x_{0}$, com $x \neq x_{0}$, pelo número generalizado invertível $\alpha_{-\ln \left\|x-x_{0}\right\|}$ (Proposição 1.1.9). Isto porque, como $\overline{\mathbb{K}}$ não é corpo (Observação 1.1.6), $x-x_{0}$ poderia não ser invertível em $\overline{\mathbb{K}}$. Eles ainda observam que não seria conveniente substituir $x-x_{0}$ por $\left\|x-x_{0}\right\|$, como é feito no contexto clássico, visto que para $f$ contínua em $x_{0}$ tería-se que

$$
0=\lim _{x \rightarrow x_{0}}\left\|f(x)-f\left(x_{0}\right)\right\|=\lim _{x \rightarrow x_{0}}\left\|f(x)-f\left(x_{0}\right)-z_{0}\left(x-x_{0}\right)\right\|, \forall z_{0} \in \overline{\mathbb{K}},
$$

onde a segunda igualdade decorre do Teorema 2.1.2 (1) e, como pela Proposição 1.3.7 (4), tem-se que

$$
\left\|\frac{f(x)-f\left(x_{0}\right)-z_{0}\left(x-x_{0}\right)}{\left\|x-x_{0}\right\|}\right\|=\left\|f(x)-f\left(x_{0}\right)-z_{0}\left(x-x_{0}\right)\right\|, \quad \forall z_{0} \in \bar{K},
$$

obtería-se que

$$
\lim _{x \rightarrow x_{0}} \frac{f(x)-f\left(x_{0}\right)-z_{0}\left(x-x_{0}\right)}{\left\|x-x_{0}\right\|}=0, \forall z_{0} \in \overline{\mathbb{K}} .
$$

Dessa forma, toda função contínua em $x_{0}$ seria derivável em $x_{0}$ e, além disso, sua derivada não seria única.

Pelo fato da ultramétrica $D$ ser invariante por translações (Teorema 1.3.8) temos que, se $U$ é um subconjunto aberto de $\overline{\mathbb{K}}, x_{0} \in U, L \in \overline{\mathbb{K}}$ e $g: U+\left\{-x_{0}\right\} \longrightarrow \overline{\mathbb{K}}$, então

$$
\lim _{x \rightarrow x_{0}} g\left(x-x_{0}\right)=L \Longleftrightarrow \lim _{h \rightarrow 0} g(h)=L .
$$


Logo, se $f: U \longrightarrow \overline{\mathbb{K}}$ e $z_{0} \in U$, temos que

$$
\lim _{x \rightarrow x_{0}} \frac{f(x)-f\left(x_{0}\right)-z_{0}\left(x-x_{0}\right)}{\alpha_{-\ln \left\|x-x_{0}\right\|}}=0 \Longleftrightarrow \lim _{h \rightarrow 0} \frac{f\left(x_{0}+h\right)-f\left(x_{0}\right)-z_{0} h}{\alpha_{-\ln \|h\|}}=0
$$

e, assim, o segundo limite da equivalência acima poderia ter sido usado na Definição 2.2.2.

O resultado, a seguir, fornece algumas definições equivalentes à derivabilidade de uma função $f$ em $x_{0}$ e serão úteis em muitas demonstrações.

Proposição 2.2.3 Sejam $U$ um subconjunto aberto de $\bar{K}, f: U \rightarrow \overline{\mathbb{K}}$ e $x_{0} \in U$. As seguintes afirmações são equivalentes:

1. a função $f$ é derivável em $x_{0}$ e $D(f)\left(x_{0}\right):=z_{0}$;

2. existe $z_{0} \in \overline{\mathbb{K}}$ tal que $\lim _{x \rightarrow x_{0}} \frac{\left\|f(x)-f\left(x_{0}\right)-z_{0}\left(x-x_{0}\right)\right\|}{\left\|x-x_{0}\right\|}=0$;

3. existe $z_{0} \in \bar{K}$ tal que $f(x)-f\left(x_{0}\right)=z_{0}\left(x-x_{0}\right)+E(x)$, com $\lim _{x \rightarrow x_{0}} \frac{E(x)}{\alpha_{-\ln \left\|x-x_{0}\right\|}}=0$;

4. existe $z_{0} \in \overline{\mathbb{K}}$ tal que $\rho: U \longrightarrow \overline{\mathbb{K}}$, dada por

$$
\rho(x):=\left\{\begin{array}{ll}
\frac{f(x)-f\left(x_{0}\right)-z_{0}\left(x-x_{0}\right)}{\alpha_{-} \ln \left\|x-x_{0}\right\|} & , \text { se } x \neq x_{0} \\
0 & , \text { se } x=x_{0}
\end{array},\right.
$$

é contínua em $x_{0}$ e tem-se que

$$
\begin{gathered}
f(x)-f\left(x_{0}\right)=z_{0}\left(x-x_{0}\right)+\rho(x) \alpha_{-\ln \left\|x-x_{0}\right\|}, \forall x \in U \backslash\left\{x_{0}\right\} ; \\
\left\|f(x)-f\left(x_{0}\right)-z_{0}\left(x-x_{0}\right)\right\|=\|\rho(x)\|\left\|x-x_{0}\right\|, \quad \forall x \in U .
\end{gathered}
$$

Demonstração: É suficiente mostrarmos que

$$
\text { 1. } 3 . \Longleftrightarrow 2 . \Longleftrightarrow 4 \text {.. }
$$

Para tanto, notemos que a primeira equivalência é obtida a partir da definição de derivabilidade de $f$ no ponto $x_{0}$. Para as demais equivalências, basta observarmos que, da 
Proposição 1.3.7 (5), temos os seguintes fatos

$$
\left\|\frac{y}{\alpha_{-\ln \left\|x-x_{0}\right\|}}\right\|=\left\|y \alpha_{\ln \left\|x-x_{0}\right\|}\right\|=\|y\| e^{-\ln \left\|x-x_{0}\right\|}=\frac{\|y\|}{\left\|x-x_{0}\right\|}
$$

e

$$
\left\|y \alpha_{-\ln \left\|x-x_{0}\right\|}\right\|=\|y\| e^{\ln \left\|x-x_{0}\right\|}=\|y\|\left\|x-x_{0}\right\|
$$

para todo $y \in \overline{\mathbb{K}}$ e todo $x \in \overline{\mathbb{K}} \backslash\left\{x_{0}\right\}$.

Exemplo 2.2.4 Seja $f: \bar{K} \longrightarrow \bar{K}$.

1. Se $f$ é constante, isto é, existe $c \in \overline{\mathbb{K}}$ tal que $f(x):=c$, para todo $x \in \bar{K}$, então $f$ é derivável em $\overline{\mathbb{K}}$ e $D(f)\left(x_{0}\right)=0$, para todo $x_{0} \in \overline{\mathbb{K}}$.

De fato, para $x_{0} \in \bar{K}$ temos que

$$
\lim _{x \rightarrow x_{0}} \frac{f(x)-f\left(x_{0}\right)-0\left(x-x_{0}\right)}{\alpha_{-\ln \left\|x-x_{0}\right\|}}=\lim _{x \rightarrow x_{0}} \frac{0}{\alpha_{-\ln \left\|x-x_{0}\right\|}}=0
$$

e, assim, $D(f)\left(x_{0}\right)=0$.

2. Se fé dada por

$$
f(x):= \begin{cases}\alpha_{-2 \ln \|x\|} & , \text { se } x \neq 0 \\ 0 & , \text { se } x=0\end{cases}
$$

então $f$ é derivável em $\overline{\mathbb{K}}$ e $D(f)\left(x_{0}\right)=0$, para todo $x_{0} \in \bar{K}$.

De fato, se $x_{0}=0$, então

$$
\lim _{x \rightarrow x_{0}} \frac{\left\|f(x)-f\left(x_{0}\right)-0\left(x-x_{0}\right)\right\|}{\left\|x-x_{0}\right\|}=\lim _{x \rightarrow 0} \frac{\left\|\alpha_{-2} \ln \right\| x\|\|}{\|x\|}=\lim _{x \rightarrow 0} \frac{\|x\|^{2}}{\|x\|}=\lim _{x \rightarrow 0}\|x\|=0,
$$

onde a segunda igualdade segue da Proposição 1.3 .7 (8). Portanto, a afirmação 2. da Proposição 2.2.3 está satisfeita e, assim, $f$ é derivável em 0 e $D(f)(0)=0$. Se $x_{0} \neq 0$, então $S_{\left\|x_{0}\right\|}(0):=\left\{x \in \overline{\mathbb{K}}:\|x\|=\left\|x_{0}\right\|\right\}$ é uma vizinhança aberta de $x_{0}$ (pois, $\left(\overline{\mathbb{K}}, \mathscr{T}_{c}\right)$ é espaço ultramétrico) na qual $f$ é constante. Logo, por 1 ., $D(f)\left(x_{0}\right)=0$. 
3. Se $f$ é dada por $f(x):=x^{n}$, onde $n \in \mathbb{N}^{*}$, então $f$ é derivável e $D(f)(x)=n x^{n-1}$, para todo $x \in \bar{K}$.

De fato, fixemos $x \in \bar{K}$. Para $n=1$ é claro que $D(f)(x)=1$. Sejam $n \geq 2$ e

$$
E(y):=f(y)-f(x)-n x^{n-1}(y-x), \forall y \in \overline{\mathbb{K}} .
$$

Para $y \in \bar{K} \backslash\{x\}$, seja $h:=y-x$. Então

$$
E(x+h)=(x+h)^{n}-x^{n}-n x^{n-1} h=\left(\sum_{j=0}^{n}\left(\begin{array}{l}
n \\
j
\end{array}\right) x^{j} h^{n-j}\right)-x^{n}-n x^{n-1} h
$$

e, assim,

$$
E(x+h)=\sum_{j=0}^{n-2}\left(\begin{array}{l}
n \\
j
\end{array}\right) x^{j} h^{n-j} .
$$

Logo, pela Proposição 1.3.7 (1), (2) e (4), temos que

$$
\|E(x+h)\|=\left\|\sum_{j=0}^{n-2}\left(\begin{array}{l}
n \\
j
\end{array}\right) x^{j} h^{n-j}\right\| \leq \max \left\{\left\|x^{j}\right\|\|h\|^{n-j}: 0 \leq j \leq n-2\right\}
$$

e, portanto,

$$
0 \leq \frac{\|E(x+h)\|}{\|h\|} \leq \max \left\{\left\|x^{j}\right\|\|h\|^{n-j-1}: 0 \leq j \leq n-2\right\} .
$$

Como

$$
\lim _{y \rightarrow x}\left(\left\|x^{j}\right\|\|y-x\|^{n-j-1}\right)=0, \quad \forall 0 \leq j \leq n-2,
$$

segue, de (2.5), que

$$
\lim _{y \rightarrow x} \frac{\|E(y)\|}{\|y-x\|}=0
$$

Logo, a afirmação 2. da Proposição 2.2 .3 é verdadeira e, portanto, $f$ é derivável em $x$ e $D(f)(x)=n x^{n-1}$.

Apresentaremos, mais adiante, uma função contínua em um ponto $x_{0}$ que não é derivável nesse ponto (Exemplo 2.2.8).

A partir do Exemplo 2.2.4 (2), podemos concluir o seguinte: 
Observação 2.2.5 Seja $f: \bar{K} \longrightarrow \bar{K}$. Tem-se que

(a) $D(f)=0$ em $\overline{\mathbb{K}}$ não implica $f$ constante;

(b) se $\mathbb{K}=\mathbb{R}$, então o Teorema do Valor Médio, neste contexto, é falso em geral.

$\mathrm{Na}$ Proposição 2.2.6 veremos que, se $f$ é derivável em $x_{0}$, então, como no contexto clássico, pode-se expressar $D(f)\left(x_{0}\right)$ como um limite.

Proposição 2.2.6 Sejam $U$ um subconjunto aberto de $\overline{\mathbb{K}}, f: U \longrightarrow \bar{K}$ e $x_{0} \in U$. Se $f$ é derivável em $x_{0}$, então

$$
D(f)\left(x_{0}\right)=\lim _{n \rightarrow \infty} \frac{f\left(x_{0}+\alpha_{n}\right)-f\left(x_{0}\right)}{\alpha_{n}} .
$$

Demonstração: Seja, para cada $n \in \mathbb{N}, x_{n}:=x_{0}+\alpha_{n}$. Observemos que, pela Proposição 1.3.7 (8), temos que

$$
\left\|x_{n}-x_{0}\right\|=\left\|\alpha_{n}\right\|=e^{-n}
$$

$\mathrm{e}$

$$
\begin{aligned}
\frac{f\left(x_{n}\right)-f\left(x_{0}\right)-D(f)\left(x_{0}\right)\left(x_{n}-x_{0}\right)}{\alpha_{-\ln \left\|x_{n}-x_{0}\right\|}} & =\frac{f\left(x_{0}+\alpha_{n}\right)-f\left(x_{0}\right)-D(f)\left(x_{0}\right) \alpha_{n}}{\alpha_{-\ln \left\|\alpha_{n}\right\|}} \\
& =\frac{f\left(x_{0}+\alpha_{n}\right)-f\left(x_{0}\right)}{\alpha_{n}}-D(f)\left(x_{0}\right),
\end{aligned}
$$

para todo $n \in \mathbb{N}$. Então $\lim _{n \rightarrow \infty} x_{n}=x_{0}$ e, assim, da derivabilidade de $f$ em $x_{0}$ e de (2.6), temos que $D(f)\left(x_{0}\right)=\lim _{n \rightarrow \infty} \frac{f\left(x_{0}+\alpha_{n}\right)-f\left(x_{0}\right)}{\alpha_{n}}$.

O próximo resultado é análogo ao do contexto clássico.

Proposição 2.2.7 Sejam $U$ um subconjunto aberto de $\overline{\mathbb{K}}$ e $x_{0} \in U$. Se $f: U \longrightarrow \bar{K}$ é derivável em $x_{0}$, então $f$ é contínua em $x_{0}$. 
Demonstração: Podemos escrever, pela Proposição 2.2.3, que

$$
f(x)-f\left(x_{0}\right)=D(f)\left(x_{0}\right)\left(x-x_{0}\right)+E(x), \quad \operatorname{com} \lim _{x \rightarrow x_{0}} \frac{E(x)}{\alpha_{-\ln \left\|x-x_{0}\right\|}}=0 .
$$

Além disso, pela Proposição 1.3.7 (5), temos que

$$
\|E(x)\|=\left\|\frac{E(x)}{\alpha_{-\ln \left\|x-x_{0}\right\|}} \alpha_{-\ln \left\|x-x_{0}\right\|}\right\|=\left\|\frac{E(x)}{\alpha_{-\ln \left\|x-x_{0}\right\|}}\right\| e^{\ln \left\|x-x_{0}\right\|}=\left\|\frac{E(x)}{\alpha_{-\ln \left\|x-x_{0}\right\|}}\right\|\left\|x-x_{0}\right\|,
$$

para todo $x \in U \backslash\left\{x_{0}\right\}$. Assim, $\lim _{x \rightarrow x_{0}} E(x)=0$ e, portanto, pelo Teorema 2.1.2 (1), obtemos que

$$
\lim _{x \rightarrow x_{0}}\left(f(x)-f\left(x_{0}\right)\right)=\lim _{x \rightarrow x_{0}}\left(D(f)\left(x_{0}\right)\left(x-x_{0}\right)+E(x)\right)=0 .
$$

Logo, $f$ é contínua em $x_{0}$.

O exemplo a seguir nos mostra que a recíproca da Proposição 2.2 .7 pode ser falsa.

Exemplo 2.2.8 Se $f: \bar{K} \longrightarrow \bar{K}$ é dada por

$$
f(x):=\left\{\begin{array}{ll}
x & , \text { se } x=\alpha_{2 n} \text { para algum } n \in \mathbb{N} \\
0, & \text { caso contrário }
\end{array},\right.
$$

então $f$ é contínua em $x_{0}=0$ e não é derivável em $x_{0}=0$.

De fato, para a continuidade de $f$ em $x_{0}=0$, basta usarmos a Proposição 1.3.7 (8) e observarmos que, se $\sigma>0$ e $n_{0} \in \mathbb{N}$ é tal que $e^{-2 n_{0}}<\sigma$, então para $x \in \bar{K}$ com $\|x\|<e^{-2 n_{0}}$ temos que $x \neq \alpha_{k}$, para $k \leq 2 n_{0}$, e, portanto,

$$
\|f(x)\| \leq \sup \left\{\left\|\alpha_{s}\right\|: s>2 n_{0}\right\}=\sup \left\{e^{-s}: s>2 n_{0}\right\}=e^{-2 n_{0}}<\sigma
$$

Sejam, para cada $n \in \mathbb{N}, x_{n}:=\alpha_{2 n}$ e $y_{n}:=\alpha_{2 n+1}$. Notemos que

$$
\lim _{n \rightarrow \infty} \frac{f\left(x_{n}\right)-f(0)}{x_{n}}=\lim _{n \rightarrow \infty} \frac{\alpha_{2 n}}{\alpha_{2 n}}=1 \quad \text { e } \quad \lim _{n \rightarrow \infty} \frac{f\left(y_{n}\right)-f(0)}{y_{n}}=\lim _{n \rightarrow \infty} \frac{0}{\alpha_{2 n+1}}=0 .
$$

Logo, o $\lim _{n \rightarrow \infty} \frac{f\left(\alpha_{n}\right)-f(0)}{\alpha_{n}}$ não existe e, assim, pela Proposição 2.2.6, concluímos que $f$ não é derivável em $x_{0}=0$. 
Veremos, a seguir, que as regras de derivação são verdadeiras neste contexto.

Proposição 2.2.9 Seja $U$ um subconjunto aberto de $\overline{\mathbb{K}}$. Se $f, g: U \longrightarrow \overline{\mathbb{K}}$ são deriváveis em $x_{0} \in U$ e $c \in \bar{K}$, ent $\tilde{a} o$

1. $f+g$ é derivável em $x_{0}$ e $D(f+g)\left(x_{0}\right)=D(f)\left(x_{0}\right)+D(g)\left(x_{0}\right)$;

2. $c f$ é derivável em $x_{0}$ e $D(c f)\left(x_{0}\right)=c D(f)\left(x_{0}\right)$;

3. $f g$ é derivável em $x_{0}$ e $D(f g)\left(x_{0}\right)=g\left(x_{0}\right) D(f)\left(x_{0}\right)+f\left(x_{0}\right) D(g)\left(x_{0}\right)$.

Demonstração: Sejam $E_{1}$ e $E_{2}$ as funções definidas em $U$ por

$E_{1}(x):=f(x)-f\left(x_{0}\right)-D(f)\left(x_{0}\right)\left(x-x_{0}\right) \quad$ e $\quad E_{2}(x):=g(x)-g\left(x_{0}\right)-D(g)\left(x_{0}\right)\left(x-x_{0}\right)$.

Pela derivabilidade de $f$ e $g$ em $x_{0}$, temos que

$$
\lim _{x \rightarrow x_{0}} \frac{E_{1}(x)}{\alpha_{-\ln \left\|x-x_{0}\right\|}}=0 \quad \text { e } \quad \lim _{x \rightarrow x_{0}} \frac{E_{2}(x)}{\alpha_{-\ln \left\|x-x_{0}\right\|}}=0,
$$

ou seja, pela Proposição $2.2 .3(2)$, que

$$
\lim _{x \rightarrow x_{0}} \frac{\left\|E_{1}(x)\right\|}{\left\|x-x_{0}\right\|}=0 \quad \text { e } \quad \lim _{x \rightarrow x_{0}} \frac{\left\|E_{2}(x)\right\|}{\left\|x-x_{0}\right\|}=0
$$

Para as duas primeiras afirmações, usando o Teorema 2.1.2 (1), basta observarmos que, se $h_{1}=f+g$ e $h_{2}=c f$, então, para $x \in U \backslash\left\{x_{0}\right\}$,

$$
\frac{h_{1}(x)-h_{1}\left(x_{0}\right)-\left(D(f)\left(x_{0}\right)+D(g)\left(x_{0}\right)\right)\left(x-x_{0}\right)}{\alpha_{-\ln \left\|x-x_{0}\right\|}}=\frac{E_{1}(x)}{\alpha_{-\ln \left\|x-x_{0}\right\|}}+\frac{E_{2}(x)}{\alpha_{-\ln \left\|x-x_{0}\right\|}}
$$

e

$$
\frac{h_{2}(x)-h_{2}\left(x_{0}\right)-\left(c D(f)\left(x_{0}\right)\right)\left(x-x_{0}\right)}{\alpha_{-\ln \left\|x-x_{0}\right\|}}=c \frac{E_{1}(x)}{\alpha_{-\ln \left\|x-x_{0}\right\|}} .
$$

Finalmente, para a última afirmação, seja $E_{3}$ a função definida em $U$ por

$$
E_{3}(x):=f(x) g(x)-f\left(x_{0}\right) g\left(x_{0}\right)-\left(g\left(x_{0}\right) D(f)\left(x_{0}\right)+f\left(x_{0}\right) D(g)\left(x_{0}\right)\right)\left(x-x_{0}\right) .
$$


Então

$$
\begin{aligned}
E_{3}(x) & =E_{1}(x) g\left(x_{0}\right)+E_{2}(x) f(x)+D(g)\left(x_{0}\right)\left(x-x_{0}\right) f(x)-f\left(x_{0}\right) D(g)\left(x_{0}\right)\left(x-x_{0}\right) \\
& =E_{1}(x) g\left(x_{0}\right)+E_{2}(x) f(x)+D(g)\left(x_{0}\right)\left(x-x_{0}\right)\left(f(x)-f\left(x_{0}\right)\right) .
\end{aligned}
$$

Logo, pela Proposição 1.3.7 (1) e (2), temos, para $x \in U \backslash\left\{x_{0}\right\}$, que

$$
\frac{\left\|E_{3}(x)\right\|}{\left\|x-x_{0}\right\|} \leq \max \left\{\frac{\left\|E_{1}(x)\right\|}{\left\|x-x_{0}\right\|}\left\|g\left(x_{0}\right)\right\|, \frac{\left\|E_{2}(x)\right\|}{\left\|x-x_{0}\right\|}\|f(x)\|,\left\|D(g)\left(x_{0}\right)\right\|\left\|f(x)-f\left(x_{0}\right)\right\|\right\} .
$$

Portanto, da continuidade de $f$ em $x_{0}$ (Proposição 2.2.7), de (2.8) e da Proposição 2.1.1 (2), concluímos que

$$
\lim _{x \rightarrow x_{0}} \frac{\left\|E_{3}(x)\right\|}{\left\|x-x_{0}\right\|}=0,
$$

o que prova 3. (Proposição 2.2.3).

No contexto clássico, se uma função real $f$ é contínua e não se anula em um ponto $x_{0}$ pertencente a $\mathbb{R}$ podemos definir, em uma vizinhança aberta de $x_{0}$, a função $\frac{1}{f}$. A seguir, apresentaremos um resultado similar, onde utilizamos o conjunto dos invertíveis de $\overline{\mathbb{K}}$, o qual, como vimos na Definição 1.1.7, é denotado por $\operatorname{Inv}(\bar{K})$.

Proposição 2.2.10 Sejam $U$ um subconjunto aberto de $\overline{\mathbb{K}}$ e $g: U \longrightarrow \bar{K}$. Se gé contínua em $x_{0} \in U$ e $g\left(x_{0}\right)$ é invertível, então existe $V$ vizinhança aberta de $x_{0}$ tal que $g(x)$ é invertivel, para todo $x \in V$.

Demonstração: Temos, pela Proposição 1.4.7, que $\operatorname{Inv}(\overline{\mathbb{K}})$ é um subconjunto aberto de $\overline{\mathbb{K}}$. Logo, como $g\left(x_{0}\right) \in \operatorname{Inv}(\bar{K})$, existe $W$ vizinhança aberta de $g\left(x_{0}\right)$ tal que $W \subset \operatorname{Inv}(\bar{K})$. Pela continuidade de $g$ em $x_{0}$, existe $V$ vizinhança aberta de $x_{0}$ tal que $g(V) \subset W$. Assim, $g(x)$ é invertível, para todo $x \in V$.

Proposição 2.2.11 Sejam $U$ um subconjunto aberto de $\bar{K}, x_{0} \in U$ e $f, g: U \longrightarrow \bar{K}$. Se $f$ e g são deriváveis em $x_{0}$ e $g\left(x_{0}\right)$ é invertivel, então $\frac{f}{g}$ é derivável em $x_{0} e$

$$
D\left(\frac{f}{g}\right)\left(x_{0}\right)=\frac{g\left(x_{0}\right) D(f)\left(x_{0}\right)-f\left(x_{0}\right) D(g)\left(x_{0}\right)}{g^{2}\left(x_{0}\right)} .
$$


Demonstração: Primeiramente, vamos mostrar que

$$
\frac{1}{g} \text { é derivável em } x_{0} \quad \text { e } \quad D\left(\frac{1}{g}\right)\left(x_{0}\right)=\frac{-D(g)\left(x_{0}\right)}{g^{2}\left(x_{0}\right)} .
$$

Seja $E_{1}$ a função definida em $U$ por

$$
E_{1}(x):=g(x)-g\left(x_{0}\right)-D(g)\left(x_{0}\right)\left(x-x_{0}\right)
$$

Como $g$ é derivável em $x_{0}$, temos, pela Proposição $2.2 .3(2)$, que

$$
\lim _{x \rightarrow x_{0}} \frac{\left\|E_{1}(x)\right\|}{\left\|x-x_{0}\right\|}=0 .
$$

Da continuidade de $g$ em $x_{0}$ (Proposição 2.2.7) e de $g\left(x_{0}\right)$ ser invertível, existe, pela Proposição 2.2.10, $V$ vizinhança aberta de $x_{0}$ tal que $g(x)$ é invertível, para todo $x \in V$. Desse modo, para todo $x \in V$, podemos definir

$$
E_{2}(x):=\frac{1}{g(x)}-\frac{1}{g\left(x_{0}\right)}-\left(-\frac{D(g)\left(x_{0}\right)}{g^{2}\left(x_{0}\right)}\left(x-x_{0}\right)\right)
$$

Então

$$
\begin{aligned}
E_{2}(x) & =\frac{g^{2}\left(x_{0}\right)-g(x) g\left(x_{0}\right)+g(x) D(g)\left(x_{0}\right)\left(x-x_{0}\right)}{g(x) g^{2}\left(x_{0}\right)} \\
& =\frac{-g\left(x_{0}\right) E_{1}(x)-g\left(x_{0}\right) D(g)\left(x_{0}\right)\left(x-x_{0}\right)+g(x) D(g)\left(x_{0}\right)\left(x-x_{0}\right)}{g(x) g^{2}\left(x_{0}\right)} \\
& =\frac{-E_{1}(x)}{g(x) g\left(x_{0}\right)}+\frac{D(g)\left(x_{0}\right)\left(x-x_{0}\right)\left(g(x)-g\left(x_{0}\right)\right)}{g(x) g^{2}\left(x_{0}\right)} .
\end{aligned}
$$

Logo, pela Proposição 1.3.7 (1), (2) e (4), temos, para $x \in V \backslash\left\{x_{0}\right\}$, que

$$
\frac{\left\|E_{2}(x)\right\|}{\left\|x-x_{0}\right\|} \leq \max \left\{\frac{\left\|E_{1}(x)\right\|}{\left\|x-x_{0}\right\|}\left\|\frac{1}{g(x) g\left(x_{0}\right)}\right\|,\left\|D(g)\left(x_{0}\right)\right\|\left\|g(x)-g\left(x_{0}\right)\right\|\left\|\frac{1}{g(x) g^{2}\left(x_{0}\right)}\right\|\right\} .
$$

Portanto, da continuidade de $g$ em $x_{0}$, de (2.10), do Teorema 2.1.3 (1) e (2) e da Proposição 2.1.1 (2), concluímos que

$$
\lim _{x \rightarrow x_{0}} \frac{\left\|E_{2}(x)\right\|}{\left\|x-x_{0}\right\|}=0
$$

o que prova (2.9) (Proposição 2.2.3). 
De (2.9) e da Proposição 2.2 .9 (3), obtemos que $f \frac{1}{g}$ é derivável em $x_{0}$ e

$$
\begin{aligned}
D\left(\frac{f}{g}\right)\left(x_{0}\right)=D\left(f \frac{1}{g}\right)\left(x_{0}\right) & =\frac{1}{g\left(x_{0}\right)} D(f)\left(x_{0}\right)+f\left(x_{0}\right) D\left(\frac{1}{g}\right)\left(x_{0}\right) \\
& =\frac{1}{g\left(x_{0}\right)} D(f)\left(x_{0}\right)-f\left(x_{0}\right) \frac{D(g)\left(x_{0}\right)}{g^{2}\left(x_{0}\right)} \\
& =\frac{g\left(x_{0}\right) D(f)\left(x_{0}\right)-f\left(x_{0}\right) D(g)\left(x_{0}\right)}{g^{2}\left(x_{0}\right)}
\end{aligned}
$$

Proposição 2.2.12 (Regra da Cadeia) Sejam $U$ e $V$ subconjuntos abertos de $\overline{\mathbb{K}}$, $f: U \longrightarrow \overline{\mathbb{K}}$ e $g: V \longrightarrow \bar{K}$, com $f(U) \subset V$. Se $f$ é derivável em $x_{0} \in U$ e $g$ é derivável em $f\left(x_{0}\right)$, então $g \circ f$ é derivável em $x_{0} e$

$$
D(g \circ f)\left(x_{0}\right)=D(g)\left(f\left(x_{0}\right)\right) D(f)\left(x_{0}\right)
$$

Demonstração: Sejam $E_{1}, E_{2}$ as funções definidas em $U$ e $V$, respectivamente, por $E_{1}(x):=f(x)-f\left(x_{0}\right)-D(f)\left(x_{0}\right)\left(x-x_{0}\right)$ e $E_{2}(y):=g(y)-g\left(f\left(x_{0}\right)\right)-D(g)\left(f\left(x_{0}\right)\right)\left(y-f\left(x_{0}\right)\right)$ e seja $E_{3}$ a função definida em $U$ por

$$
E_{3}(x):=g(f(x))-g\left(f\left(x_{0}\right)\right)-D(g)\left(f\left(x_{0}\right)\right) D(f)\left(x_{0}\right)\left(x-x_{0}\right) .
$$

Para concluirmos a prova, é suficiente, pela Proposição 2.2.3, mostrarmos que

$$
\lim _{x \rightarrow x_{0}} \frac{\left\|E_{3}(x)\right\|}{\left\|x-x_{0}\right\|}=0 .
$$

Notemos que

$$
\begin{aligned}
E_{3}(x) & =E_{2}(f(x))+D(g)\left(f\left(x_{0}\right)\right)\left(f(x)-f\left(x_{0}\right)-D(f)\left(x_{0}\right)\left(x-x_{0}\right)\right) \\
& =E_{2}(f(x))+D(g)\left(f\left(x_{0}\right)\right) E_{1}(x)
\end{aligned}
$$


para $x \in U$. Então, para $x \in U \backslash\left\{x_{0}\right\}$, temos, pela Proposição 1.3 .7 (1) e (2), que

$$
\frac{\left\|E_{3}(x)\right\|}{\left\|x-x_{0}\right\|} \leq \max \left\{\frac{\left\|E_{2}(f(x))\right\|}{\left\|x-x_{0}\right\|},\left\|D(g)\left(f\left(x_{0}\right)\right)\right\| \frac{\left\|E_{1}(x)\right\|}{\left\|x-x_{0}\right\|}\right\} \text {. }
$$

Da derivabilidade de $f$ em $x_{0}$ e de $g$ em $f\left(x_{0}\right)$, temos, pela Proposição 2.2.3, que

$$
\begin{gathered}
\lim _{x \rightarrow x_{0}} \frac{\left\|E_{1}(x)\right\|}{\left\|x-x_{0}\right\|}=0 ; \\
\text { existe } \rho: V \longrightarrow \overline{\mathbb{K}} \text { contínua em } f\left(x_{0}\right) \text {, tal que } \rho\left(f\left(x_{0}\right)\right)=0
\end{gathered}
$$

e

$$
\left\|E_{2}(y)\right\|=\|\rho(y)\|\left\|y-f\left(x_{0}\right)\right\|, \forall y \in V .
$$

Logo, de (2.11) e (2.12), para concluirmos a prova, basta provarmos que

$$
\lim _{x \rightarrow x_{0}} \frac{\left\|E_{2}(f(x))\right\|}{\left\|x-x_{0}\right\|}=0
$$

Para isso, observemos que, de (2.14), para $x \in U \backslash\left\{x_{0}\right\}$, temos

$$
\begin{aligned}
\frac{\left\|E_{2}(f(x))\right\|}{\left\|x-x_{0}\right\|} & =\|\rho(f(x))\| \frac{\left\|f(x)-f\left(x_{0}\right)\right\|}{\left\|x-x_{0}\right\|} \\
& =\|\rho(f(x))\| \frac{\left\|E_{1}(x)+D(f)\left(x_{0}\right)\left(x-x_{0}\right)\right\|}{\left\|x-x_{0}\right\|} \\
& \leq\|\rho(f(x))\| \max \left\{\frac{\left\|E_{1}(x)\right\|}{\left\|x-x_{0}\right\|},\left\|D(f)\left(x_{0}\right)\right\|\right\},
\end{aligned}
$$

onde a última desigualdade decorre da Proposição 1.3 .7 (1) e (2). Assim, de (2.12), de (2.13), da continuidade de $f$ em $x_{0}$ (Proposição 2.2.7) e da Proposição 2.1.1 (2), obtemos

$$
\lim _{x \rightarrow x_{0}} \frac{\left\|E_{2}(f(x))\right\|}{\left\|x-x_{0}\right\|}=0
$$

No capítulo 3, discutiremos com mais detalhes as funções definidas em um subconjunto aberto de $\overline{\mathbb{C}}$ e a valores em $\overline{\mathbb{C}}$ e provaremos, entre outros resultados, que valem as Condições de Cauchy-Riemann neste contexto.

Nas próximas seções, consideraremos $\mathbb{K}=\mathbb{R}$ e introduziremos o conceito de diferenciabilidade para funções definidas em subconjuntos abertos de $\overline{\mathbb{R}}^{n}$ e a valores em $\overline{\mathbb{R}}$. 


\subsection{Diferenciabilidade: funções de várias variáveis reais generalizadas}

Nesta seção são estudados, para funções definidas em subconjuntos abertos de $\overline{\mathbb{R}}^{n} \mathrm{e}$ a valores em $\overline{\mathbb{R}}$, os conceitos de derivadas parciais e de diferenciabilidade. Existe, de modo similar ao do contexto clássico, uma relação entre esses conceitos (Proposição 2.3.5).

Para cada $1 \leq i \leq n$, denotaremos por $e_{i}$ o elemento de $\overline{\mathbb{R}}^{n}$ dado por $e_{i}:=\left[\left(\mu_{i}\right)_{\varepsilon}\right]$, onde $\mu_{i}$ é o i-ésimo elemento da base canônica de $\mathbb{R}^{n}$. É fácil verificar que $\overline{\mathbb{R}}^{n}$, como $\overline{\mathbb{R}}$-módulo, tem $\left\{e_{i}: 1 \leq i \leq n\right\}$ como base. Essa base será chamada de base canônica de $\overline{\mathbb{R}}^{n}$.

A fim de fixarmos a notação, lembramos que, se $x$ é um elemento qualquer de $\overline{\mathbb{R}}^{n}$, então sua i-ésima coordenada é denotada por $x_{i}$ e temos que $\|x\|_{n}:=\max \left\{\left\|x_{i}\right\|: 1 \leq i \leq n\right\}$. Com a finalidade de simplificarmos a notação, denotaremos $\|\cdot\|_{n}$ por $\|\cdot\|$, a menos de menção em contrário.

A seguir, apresentaremos um resultado que nos permitirá definir derivadas parciais neste contexto.

Lema 2.3.1 Sejam $U$ um subconjunto aberto de $\overline{\mathbb{R}}^{n},\left\{e_{i}: 1 \leq i \leq n\right\}$ a base canônica de $\overline{\mathbb{R}}^{n}, f: U \longrightarrow \mathbb{\mathbb { R }}$ e $x_{0} \in U$. Então, para cada $1 \leq i \leq n$, existe no máximo um $a_{i} \in \mathbb{\mathbb { R }}$ tal que

$$
\lim _{h \rightarrow 0} \frac{f\left(x_{0}+h e_{i}\right)-f\left(x_{0}\right)-a_{i} h}{\alpha_{-\ln \|h\|}}=0 .
$$

Demonstração: Sejam $1 \leq i \leq n$ e $a_{i}, b_{i} \in \overline{\mathbb{R}}$ tais que

$$
\lim _{h \rightarrow 0} \frac{f\left(x_{0}+h e_{i}\right)-f\left(x_{0}\right)-a_{i} h}{\alpha_{-\ln \|h\|}}=0 \quad \text { e } \quad \lim _{h \rightarrow 0} \frac{f\left(x_{0}+h e_{i}\right)-f\left(x_{0}\right)-b_{i} h}{\alpha_{-\ln \|h\|}}=0 .
$$


Então, pelo Teorema 2.1.2 (1), obtemos que

$$
\lim _{h \rightarrow 0} \frac{\left(b_{i}-a_{i}\right) h}{\alpha_{-} \ln \|h\|}=0 .
$$

Consideremos a seqüência $\left(\alpha_{m}\right)_{m \in N}$ em $\overline{\mathbb{R}}$. Temos, da Proposição 1.3 .7 (8), que $\lim _{m \rightarrow \infty}\left\|\alpha_{m}\right\|=\lim _{m \rightarrow \infty} e^{-m}=0$ e, portanto, de (2.15), obtemos que

$$
0=\lim _{m \rightarrow \infty} \frac{\left(b_{i}-a_{i}\right) \alpha_{m}}{\alpha_{-\ln \left\|\alpha_{m}\right\|}}=\lim _{m \rightarrow \infty} \frac{\left(b_{i}-a_{i}\right) \alpha_{m}}{\alpha_{m}}=b_{i}-a_{i} .
$$

Logo, $b_{i}=a_{i}$.

Definição 2.3.2 Sejam $U$ um subconjunto aberto de $\overline{\mathbb{R}}^{n},\left\{e_{i}: 1 \leq i \leq n\right\}$ a base canônica de $\overline{\mathbb{R}}^{n}, f: U \longrightarrow \overline{\mathbb{R}}$ e $x_{0} \in U$. Se $1 \leq i \leq n$ e existe $a_{i} \in \overline{\mathbb{R}}$ tal que

$$
\lim _{h \rightarrow 0} \frac{f\left(x_{0}+h e_{i}\right)-f\left(x_{0}\right)-a_{i} h}{\alpha_{-\ln \|h\|}}=0
$$

chama-se de derivada parcial de $f$, com relação a $x_{i}$, no ponto $x_{0}$, e denota-se por $\frac{\partial f}{\partial x_{i}}\left(x_{0}\right)$, o elemento de $\overline{\mathbb{R}}$ dado por $\frac{\partial f}{\partial x_{i}}\left(x_{0}\right):=a_{i}$, onde $x_{i}$ denota a $i$-ésima coordenada de $x$.

Antes de apresentarmos o conceito de diferenciabilidade, notemos que o seguinte resultado é verdadeiro:

Lema 2.3.3 Sejam $U$ um subconjunto aberto de $\overline{\mathbb{R}}^{n}, f: U \longrightarrow \overline{\mathbb{R}}$ e $x_{0}=\left(x_{01}, \ldots, x_{0 n}\right)$ $\in U$. Então existe no máximo um $a=\left(a_{1}, \ldots, a_{n}\right) \in \overline{\mathbb{R}}^{n}$ tal que

$$
\lim _{x \rightarrow x_{0}} \frac{f(x)-f\left(x_{0}\right)-\sum_{i=1}^{n} a_{i}\left(x_{i}-x_{0 i}\right)}{\alpha_{-} \ln \left\|x-x_{0}\right\|}=0
$$

onde $x_{i}$ denota a i-ésima coordenada de $x$.

Demonstração: Sejam $a=\left(a_{1}, \ldots, a_{n}\right), b=\left(b_{1}, \ldots, b_{n}\right) \in \overline{\mathbb{R}}^{n}$ tais que

$$
\lim _{x \rightarrow x_{0}} \frac{f(x)-f\left(x_{0}\right)-\sum_{i=1}^{n} a_{i}\left(x_{i}-x_{0 i}\right)}{\alpha_{-\ln \left\|x-x_{0}\right\|}}=0 \quad \text { e } \quad \lim _{x \rightarrow x_{0}} \frac{f(x)-f\left(x_{0}\right)-\sum_{i=1}^{n} b_{i}\left(x_{i}-x_{0 i}\right)}{\alpha_{-\ln \left\|x-x_{0}\right\|}}=0 .
$$


Logo, pelo Teorema 2.1.2 (1), obtemos que

$$
\lim _{x \rightarrow x_{0}} \frac{\sum_{i=1}^{n}\left(b_{i}-a_{i}\right)\left(x_{i}-x_{0 i}\right)}{\alpha_{-\ln }\left\|x-x_{0}\right\|}=0 .
$$

Seja $\left\{e_{i}: 1 \leq i \leq n\right\}$ a base canônica de $\overline{\mathbb{R}}^{n}$. Fixemos $1 \leq j \leq n$ e consideremos a seqüência $\left(x_{m}\right)_{m \in N}$, onde $x_{m}=\left(x_{m 1}, \ldots, x_{m n}\right):=x_{0}+\alpha_{m} e_{j}$. Então, pela Proposição 1.3.7 (8), temos que

$$
\lim _{m \rightarrow \infty}\left\|x_{m}-x_{0}\right\|=\lim _{m \rightarrow \infty} \max \left\{\left\|x_{m i}-x_{0 i}\right\|: 1 \leq i \leq n\right\}=\lim _{m \rightarrow \infty}\left\|\alpha_{m}\right\|=\lim _{m \rightarrow \infty} e^{-m}=0
$$

e, assim, de (2.16), concluímos que

$$
0=\lim _{m \rightarrow \infty} \frac{\sum_{i=1}^{n}\left(b_{i}-a_{i}\right)\left(x_{m i}-x_{0 i}\right)}{\alpha_{-} \ln \left\|x_{m}-x_{0}\right\|}=\lim _{m \rightarrow \infty} \frac{\left(b_{j}-a_{j}\right) \alpha_{m}}{\alpha_{m}}=b_{j}-a_{j},
$$

ou seja, $b_{j}=a_{j}$. Portanto, $a=b$.

Definição 2.3.4 Sejam $U$ um subconjunto aberto de $\overline{\mathbb{R}}^{n}, f: U \longrightarrow \overline{\mathbb{R}}$ e $x_{0}=\left(x_{01}, \ldots, x_{0 n}\right)$ $\in U$. Diz-se que $f$ é diferenciável em $x_{0}$ se existe $a=\left(a_{1}, \ldots, a_{n}\right) \in \overline{\mathbb{R}}^{n}$ tal que

$$
\lim _{x \rightarrow x_{0}} \frac{f(x)-f\left(x_{0}\right)-\sum_{i=1}^{n} a_{i}\left(x_{i}-x_{0 i}\right)}{\alpha_{-} \ln \left\|x-x_{0}\right\|}=0,
$$

onde $x_{i}$ denota a i-ésima coordenada de $x$. Diz-se que $f$ é diferenciável em $U$, se $f$ é diferenciável em cada ponto de $U$.

Do Lema 2.3.3, tem-se que, se $f$ é diferenciável em um ponto $x_{0}$, então existe um único $a=\left(a_{1}, \ldots, a_{n}\right) \in \overline{\mathbb{R}}^{n}$ satisfazendo o limite da Definição 2.3.4.

Em seguida, veremos que, como no contexto clássico, existe uma relação entre diferenciabilidade e derivadas parcias.

Proposição 2.3.5 Sejam $U$ um subconjunto aberto de $\overline{\mathbb{R}}^{n}, f: U \longrightarrow \overline{\mathbb{R}}$ e $x_{0} \in U$. As seguintes afirmações são equivalentes: 
1. $f$ é diferenciável em $x_{0}$;

2. para cada $1 \leq j \leq n$, existe $\frac{\partial f}{\partial x_{j}}\left(x_{0}\right)$ e, se $\left(a_{1}, \ldots, a_{n}\right):=\left(\frac{\partial f}{\partial x_{1}}\left(x_{0}\right), \ldots, \frac{\partial f}{\partial x_{n}}\left(x_{0}\right)\right) e$ $x_{0}=\left(x_{01}, \ldots, x_{0 n}\right)$, então

$$
\lim _{x \rightarrow x_{0}} \frac{f(x)-f\left(x_{0}\right)-\sum_{i=1}^{n} a_{i}\left(x_{i}-x_{0 i}\right)}{\alpha_{-} \ln \left\|x-x_{0}\right\|}=0,
$$

onde $x_{i}$ denota a i-ésima coordenada de $x$.

Demonstração: É imediato que 2. implica 1.. Provaremos que 1. implica $2 .$.

Seja $\left\{e_{i}: 1 \leq i \leq n\right\}$ a base canônica de $\overline{\mathbb{R}}^{n}$ e fixemos $1 \leq j \leq n$. Da diferenciabilidade de $f$ em $x_{0}$, existe $\left(a_{1}, \ldots, a_{n}\right) \in \overline{\mathbb{R}}^{n}$ tal que

$$
\lim _{x \rightarrow x_{0}} \frac{f(x)-f\left(x_{0}\right)-\sum_{i=1}^{n} a_{i}\left(x_{i}-x_{0 i}\right)}{\alpha_{-} \ln \left\|x-x_{0}\right\|}=0 .
$$

Consideremos $v=\left(v_{1}, \ldots, v_{n}\right):=x_{0}+h e_{j} \in \overline{\mathbb{R}}^{n}$, com $h \in \overline{\mathbb{R}}$ e $h \neq 0$. Então

$$
0=\lim _{h \rightarrow 0} \frac{f\left(x_{0}+h e_{j}\right)-f\left(x_{0}\right)-\sum_{i=1}^{n} a_{i}\left(v_{i}-x_{0 i}\right)}{\alpha_{-\ln \|h\|}}=\lim _{h \rightarrow 0} \frac{f\left(x_{0}+h e_{j}\right)-f\left(x_{0}\right)-a_{j} h}{\alpha_{-} \ln \|h\|},
$$

ou seja, $a_{j}=\frac{\partial f}{\partial x_{j}}\left(x_{0}\right)$.

O próximo resultado nos mostra que diferenciabilidade implica continuidade.

Proposição 2.3.6 Sejam $U$ um subconjunto aberto de $\overline{\mathbb{R}}^{n}, f: U \longrightarrow \overline{\mathbb{R}}$ e $x_{0} \in U$. Se $f$ é diferenciável em $x_{0}$, então $f$ é contínua em $x_{0}$.

Demonstração: Da diferenciabilidade de $f$ em $x_{0}=\left(x_{01}, \ldots, x_{0 n}\right)$, existe $a=\left(a_{1}, \ldots, a_{n}\right) \in$ $\overline{\mathbb{R}}^{n}$ tal que

$$
\lim _{x \rightarrow x_{0}} \frac{E(x)}{\alpha_{-\ln \left\|x-x_{0}\right\|}}=0
$$

onde

$$
E(x):=f(x)-f\left(x_{0}\right)-\sum_{i=1}^{n} a_{i}\left(x_{i}-x_{0 i}\right), \forall x=\left(x_{1}, \ldots, x_{n}\right) \in U
$$


Então, pela Proposição 1.3.7 (8), temos que

$$
\|E(x)\|=\left\|\frac{E(x)}{\alpha_{-\ln \left\|x-x_{0}\right\|}} \alpha_{-\ln \left\|x-x_{0}\right\|}\right\|=\left\|\frac{E(x)}{\alpha_{-\ln \left\|x-x_{0}\right\|}}\right\| e^{\ln \left\|x-x_{0}\right\|}=\left\|\frac{E(x)}{\alpha_{-\ln \left\|x-x_{0}\right\|}}\right\|\left\|x-x_{0}\right\|,
$$

para todo $x \in U \backslash\left\{x_{0}\right\}$. Logo, $\lim _{x \rightarrow x_{0}} E(x)=0$ e, portanto, pelo Teorema $2.1 .2(1)$, obtemos que

$$
\lim _{x \rightarrow x_{0}}\left(f(x)-f\left(x_{0}\right)\right)=\lim _{x \rightarrow x_{0}}\left(E(x)+\sum_{i=1}^{n} a_{i}\left(x_{i}-x_{0 i}\right)\right)=0,
$$

o que conclui a prova.

No contexto clássico, sabe-se que derivadas parciais contínuas implicam função diferenciável. Não há registro, até o momento, se esse resultado é verdadeiro ou não no contexto aqui abordado.

De modo similar ao do contexto clássico, define-se, para $U$ subconjunto aberto de $\overline{\mathbb{R}}^{n}$ e $k \in \mathbb{N}$, os conjuntos

$$
\begin{gathered}
\mathscr{C}(U, \overline{\mathbb{R}}):=\{f: U \longrightarrow \overline{\mathbb{R}}: f \text { é contínua em } U\} ; \\
\mathscr{C}^{k}(U, \overline{\mathbb{R}}):=\left\{f: U \longrightarrow \overline{\mathbb{R}} \mid \partial^{\alpha} f \in \mathscr{C}(U, \overline{\mathbb{R}}), \forall \alpha \in \mathbb{N}^{n} \text { tal que } 0 \leq|\alpha| \leq k\right\} \\
\mathscr{C}^{\infty}(U, \overline{\mathbb{R}}):=\bigcap_{k \in N} \mathscr{C}^{k}(U, \overline{\mathbb{R}}) ;
\end{gathered}
$$

e para $f: U \longrightarrow \mathbb{R}$ tal que existem as derivadas parciais de $f$ em $x_{0} \in U$, define-se o gradiente de $f$ em $x_{0}$ por

$$
\nabla f\left(x_{0}\right):=\left(\frac{\partial f}{\partial x_{1}}\left(x_{0}\right), \ldots, \frac{\partial f}{\partial x_{n}}\left(x_{0}\right)\right)
$$

Na próxima seção, o conceito de diferenciabilidade será estendido para aplicações definidas em um subconjunto aberto de $\overline{\mathbb{R}}^{n}$ e a valores em $\overline{\mathbb{R}}^{m}$. 


\subsection{Diferenciabilidade: aplicações de variáveis reais ge- neralizadas}

Nesta seção, o conceito de diferenciabilidade será naturalmente estendido para funções definidas em subconjuntos abertos de $\overline{\mathbb{R}}^{n}$ e a valores de $\overline{\mathbb{R}}^{m}$. Esse conceito é equivalente à existência de uma aplicação $\overline{\mathbb{R}}$-linear que satisfaz uma condição similar à do contexto clássico (Proposição 2.4.2).

Definição 2.4.1 Sejam $U$ um subconjunto aberto de $\overline{\mathbb{R}}^{n}, f=\left(f_{1}, \ldots, f_{m}\right): U \longrightarrow \overline{\mathbb{R}}^{m} e$ $x_{0} \in U$. Diz-se que $f$ é diferenciável em $x_{0}$ se, e somente se, $f_{i}$ é diferenciável em $x_{0}$, para todo $1 \leq i \leq m$.

Proposição 2.4.2 Sejam $U$ um subconjunto aberto de $\overline{\mathbb{R}}^{n}, f=\left(f_{1}, \ldots, f_{m}\right): U \longrightarrow \overline{\mathbb{R}}^{m}$ e $x_{0} \in U$. As seguintes afirmações são equivalentes:

1. $f$ é diferenciável em $x_{0}$;

2. existe uma aplicação $\overline{\mathbb{R}}$-linear $T: \overline{\mathbb{R}}^{n} \longrightarrow \overline{\mathbb{R}}^{m}$ tal que

$$
\lim _{x \rightarrow x_{0}} \frac{f(x)-f\left(x_{0}\right)-T\left(x-x_{0}\right)}{\alpha_{-\ln \left\|x-x_{0}\right\|}}=0 .
$$

Além disso, se existe T satisfazendo 2., então essa aplicação é única e será denotada por $D(f)\left(x_{0}\right)$.

Demonstração: Primeiramente, suponhamos 1. verdadeira. Fixemos $1 \leq i \leq m$. Então existe $a_{i}=\left(a_{i 1}, \ldots, a_{i n}\right) \in \overline{\mathbb{R}}^{n}$ tal que

$$
\lim _{x \rightarrow x_{0}} \frac{f_{i}(x)-f_{i}\left(x_{0}\right)-\sum_{j=1}^{n} a_{i j}\left(x_{j}-x_{0 j}\right)}{\alpha_{-\ln \left\|x-x_{0}\right\|}}=0,
$$


onde $x_{j}$ e $x_{0 j}$ denotam, respectivamente, a j-ésima coordenada de $x$ e $x_{0}$.

Seja

$$
T_{i}(h):=\sum_{j=1}^{n} a_{i j} h_{j}, \forall h=\left(h_{1}, \ldots, h_{n}\right) \in \overline{\mathbb{R}}^{n} .
$$

Notemos que, se $\lambda \in \overline{\mathbb{R}}$ e $y=\left(y_{1}, \ldots, y_{n}\right), z=\left(z_{1}, \ldots, z_{n}\right) \in \overline{\mathbb{R}}^{n}$, então

$$
T_{i}(\lambda y+z)=\sum_{j=1}^{n} a_{i j}\left(\lambda y_{j}+z_{j}\right)=\sum_{j=1}^{n}\left(\lambda a_{i j} y_{j}+a_{i j} z_{j}\right)=\lambda \sum_{j=1}^{n} a_{i j} y_{j}+\sum_{j=1}^{n} a_{i j} z_{j}
$$

e, assim,

$$
T_{i}(\lambda y+z)=\lambda T_{i}(y)+T_{i}(z)
$$

Logo, $T_{i}$ é uma aplicação $\overline{\mathbb{R}}$-linear.

Consideremos a aplicação $T: \overline{\mathbb{R}}^{n} \longrightarrow \overline{\mathbb{R}}^{m}$ dada por

$$
T(h)=\left(T_{1}(h), \ldots, T_{m}(h)\right)
$$

É claro que $T$ é uma aplicação $\overline{\mathbb{R}}$-linear, visto que $T_{i}$ é $\overline{\mathbb{R}}$-linear, para todo $1 \leq i \leq m$.

Seja $E(x):=f(x)-f\left(x_{0}\right)-T\left(x-x_{0}\right)$, para todo $x \in U$. Então, por (2.2),

$$
\lim _{x \rightarrow x_{0}} \frac{E(x)}{\alpha_{-\ln \left\|x-x_{0}\right\|}}=0,
$$

pois a i-ésima coordenada de $\frac{E(x)}{\alpha_{-} \ln \left\|x-x_{0}\right\|}$ é da forma

$$
\frac{f_{i}(x)-f_{i}\left(x_{0}\right)-\sum_{j=1}^{n} a_{i j}\left(x_{j}-x_{0 j}\right)}{\alpha_{-\ln \left\|x-x_{0}\right\|}}
$$

e (2.17) é verdadeira. Logo, 2. está satisfeita.

A seguir, mostraremos que 2. implica 1..

Seja $T=\left(T_{1}, \ldots, T_{m}\right): \overline{\mathbb{R}}^{n} \longrightarrow \overline{\mathbb{R}}^{m}$ uma aplicação $\overline{\mathbb{R}}$-linear como em 2. , isto é, tal que

$$
\lim _{x \rightarrow x_{0}} \frac{f(x)-f\left(x_{0}\right)-T\left(x-x_{0}\right)}{\alpha_{-} \ln \left\|x-x_{0}\right\|}=0
$$

e, assim, por (2.2), temos que

$$
\lim _{x \rightarrow x_{0}} \frac{f_{i}(x)-f_{i}\left(x_{0}\right)-T_{i}\left(x-x_{0}\right)}{\alpha_{-\ln \left\|x-x_{0}\right\|}}=0, \forall 1 \leq i \leq m .
$$


Fixemos $1 \leq i \leq m$ e seja $a^{i}:=\left(T_{i}\left(e_{1}\right), \ldots, T_{i}\left(e_{n}\right)\right)$, onde $\left\{e_{j}: 1 \leq j \leq n\right\}$ é a base canônica de $\overline{\mathbb{R}}^{n}$. Então, de $(2.18)$ e da $\overline{\mathbb{R}}$-linearidade de $T$, temos

$$
\begin{aligned}
0=\lim _{x \rightarrow x_{0}} \frac{f_{i}(x)-f_{i}\left(x_{0}\right)-T_{i}\left(x-x_{0}\right)}{\alpha_{-\ln \left\|x-x_{0}\right\|}} & =\lim _{x \rightarrow x_{0}} \frac{f_{i}(x)-f_{i}\left(x_{0}\right)-T_{i}\left(\sum_{j=1}^{n}\left(x_{j}-x_{0 j}\right) e_{j}\right)}{\alpha_{-\ln \left\|x-x_{0}\right\|}} \\
& =\lim _{x \rightarrow x_{0}} \frac{f_{i}(x)-f_{i}\left(x_{0}\right)-\sum_{j=1}^{n}\left(x_{j}-x_{0 j}\right) T_{i}\left(e_{j}\right)}{\alpha_{-\ln \left\|x-x_{0}\right\|}},
\end{aligned}
$$

ou seja, $f_{i}$ é diferenciável em $x_{0}$. Logo, $f$ é diferenciável em $x_{0}$.

Finalmente, vamos mostrar que existe uma única aplicação $T$ satisfazendo 2. .

Seja $G: \overline{\mathbb{R}}^{n} \longrightarrow \overline{\mathbb{R}}^{m}$ uma outra aplicação $\overline{\mathbb{R}}$-linear tal que

$$
\lim _{x \rightarrow x_{0}} \frac{f(x)-f\left(x_{0}\right)-G\left(x-x_{0}\right)}{\alpha_{-\ln }\left\|x-x_{0}\right\|}=0
$$

então, por (2.2) e pelo Teorema 2.1.2 (1), obtemos que

$$
\lim _{x \rightarrow x_{0}} \frac{(G-T)\left(x-x_{0}\right)}{\alpha_{-\ln }\left\|x-x_{0}\right\|}=0 .
$$

Da $\overline{\mathbb{R}}$-linearidade de $G$ e $T$ e do fato de $\left\{e_{j}: 1 \leq j \leq n\right\}$ ser uma base de $\overline{\mathbb{R}}^{n}$, é suficiente provarmos que $G\left(e_{j}\right)=T\left(e_{j}\right)$, para todo $1 \leq j \leq n$.

Fixemos $1 \leq j \leq n$ e consideremos a seqüência $\left(h_{r}\right)_{r \in N}$, onde $h_{r}=\left(h_{r 1}, \ldots, h_{r n}\right):=$ $\alpha_{r} e_{j}$. Então, pela Proposição 1.3.7 (8), temos que

$$
\lim _{r \rightarrow \infty}\left\|h_{r}\right\|=\lim _{r \rightarrow \infty} \max \left\{\left\|h_{r i}\right\|: 1 \leq i \leq n\right\}=\lim _{r \rightarrow \infty}\left\|\alpha_{r}\right\|=\lim _{r \rightarrow \infty} e^{-r}=0 .
$$

Portanto, de (2.19) e da $\bar{R}$-linearidade de $G$ e $T$, temos

$$
0=\lim _{r \rightarrow \infty} \frac{(G-T)\left(h_{r}\right)}{\alpha_{-\ln \left\|h_{r}\right\|}}=\lim _{r \rightarrow \infty} \frac{(G-T)\left(\alpha_{r} e_{j}\right)}{\alpha_{r}}=\lim _{r \rightarrow \infty} \frac{\alpha_{r}(G-T)\left(e_{j}\right)}{\alpha_{r}}=(G-T)\left(e_{j}\right),
$$

ou seja, $G\left(e_{j}\right)=T\left(e_{j}\right)$. Portanto, $G=T$.

Da Proposição 2.3.5 e da prova da Proposição 2.4.2, temos o seguinte: 
Observação 2.4.3 Sejam $U$ um subconjunto aberto de $\overline{\mathbb{R}}^{n}, f=\left(f_{1}, \ldots, f_{m}\right): U \longrightarrow \overline{\mathbb{R}}^{m}$ e $x_{0} \in U$. Se $f$ é diferenciável em $x_{0}$, então a aplicação $\overline{\mathbb{R}}$-linear $D(f)\left(x_{0}\right): \overline{\mathbb{R}}^{n} \longrightarrow \overline{\mathbb{R}}^{m}$ é dada por

$$
D(f)\left(x_{0}\right)\left(h_{1}, \ldots, h_{n}\right)=\left(\sum_{j=1}^{n} \frac{\partial f_{1}}{\partial x_{j}}\left(x_{0}\right) h_{j}, \sum_{j=1}^{n} \frac{\partial f_{2}}{\partial x_{j}}\left(x_{0}\right) h_{j}, \ldots, \sum_{j=1}^{n} \frac{\partial f_{m}}{\partial x_{j}}\left(x_{0}\right) h_{j}\right) .
$$

Em virtude da Observação 2.4.3, de modo similar ao do contexto clássico, obtemos a seguinte definição:

Sejam $U$ um subconjunto aberto de $\overline{\mathbb{R}}^{n}, f=\left(f_{1}, \ldots, f_{m}\right): U \longrightarrow \overline{\mathbb{R}}^{m}$ e $x_{0} \in U$. Se $f$ é diferenciável em $x_{0}$, chama-se de matriz Jacobiana de $f$ em $x_{0}$, e denota-se por $J f\left(x_{0}\right)$, a matriz associada a $D(f)\left(x_{0}\right)$ com relação às bases canônicas de $\overline{\mathbb{R}}^{n}$ e $\overline{\mathbb{R}}^{m}$, isto é,

$$
J f\left(x_{0}\right):=\left(\begin{array}{ccc}
\frac{\partial f_{1}}{\partial x_{1}} & \cdots & \frac{\partial f_{1}}{\partial x_{n}} \\
\vdots & & \vdots \\
\frac{\partial f_{m}}{\partial x_{1}} & \cdots & \frac{\partial f_{m}}{\partial x_{n}}
\end{array}\right)
$$




\section{Capítulo 3}

\section{Funções holomorfas e funções analíticas}

Neste capítulo, damos continuidade ao estudo iniciado na seção 2.2 (caso $\mathbb{K}=\mathbb{C}$ ), na qual foi introduzido o conceito de funções deriváveis em um subconjunto aberto $U$ de $\overline{\mathbb{C}}$ e a valores em $\overline{\mathscr{C}}$. Essas funções serão chamadas de funções holomorfas em $U$ (Definição 3.2.5). De modo análogo ao contexto clássico, define-se funções analíticas em $U$ (Definição 3.2.8) e prova-se que toda função analítica em $U$ é holomorfa em $U$. Não há registro, até o momento, da equivalência (que existe no contexto clássico) entre os conceitos de função holomorfa e de função analítica. Contudo, no capítulo 4, veremos que há um conjunto significativo de funções para o qual os dois conceitos são equivalentes.

Os resultados apresentados na seção 3.2, com exceção da Proposição 3.2.1, Proposição 3.2.3, Proposição 3.2.4 e Proposição 3.2.7, são devido a J. Aragona, R. Fernandez e S. O. Juriaans ([AFJ-1]). Essas proposições, que surgiram durante o estudo do artigo [AFJ-1], nos fornecem resultados similares aos do contexto clássico relacionados às condições de Cauchy-Riemann. Para prová-las, seguimos os passos do contexto clássico. Isso nos levou a identificar $\overline{\mathbb{C}} \operatorname{com} \overline{\mathbb{R}}^{2}$ e com $\overline{\mathbb{R}}+i \overline{\mathbb{R}}$. Essa identificação é apresentada na seção 3.1 .

Nas seções 3.1 e 3.2 , iremos trabalhar simultaneamente com os conjuntos $\bar{R}$ e $\overline{\mathscr{C}}$. Por esse motivo, escreveremos 
- $[\cdot]_{\overline{\mathbb{R}}}$ e $[\cdot]_{\overline{\mathbb{C}}}$ para indicar a classe em $\overline{\mathbb{R}}$ e em $\overline{\mathbb{C}}$, respectivamente;

- $\|\cdot\|_{\overline{\boldsymbol{R}}}$ e $\|\cdot\|_{\overline{\mathbb{C}}}$ para indicar a aplicação $\|\cdot\|: \bar{K} \longrightarrow \mathbb{R}_{+}$no caso $\mathbb{K}=\mathbb{R}$ e $\mathbb{K}=\mathbb{C}$, respectivamente;

- $\|\cdot\|_{\overline{\boldsymbol{R}}^{2}}$ para indicar a aplicação $\|\cdot\|_{2}: \overline{\mathbb{R}}^{2} \longrightarrow \mathbb{R}_{+}$;

- $\approx_{\bar{R}}$ e $\approx_{\overline{\mathbb{C}}}$ para indicar a relação de associação em $\overline{\mathbb{R}}$ e em $\overline{\mathbb{C}}$, respectivamente;

- $\alpha_{r}^{*}:=\left[\left(\varepsilon^{r}\right)_{\varepsilon}\right]_{\bar{R}}$ e $\alpha_{r}:=\left[\left(\varepsilon^{r}\right)_{\varepsilon}\right]_{\bar{C}}$.

\subsection{Dois conjuntos isometricamente isomorfos a $\overline{\mathbb{C}}$}

Nesta seção, provaremos que o espaço métrico $\left(\overline{\mathbb{C}},\|\cdot\|_{\overline{\mathbb{C}}}\right)$ é isometricamente isomorfo a $\left(\overline{\mathbb{R}}^{2},\|\cdot\|_{\bar{R}^{2}}\right)$. Além disso, muniremos $\overline{\mathbb{R}}+i \overline{\mathbb{R}}$ com uma métrica de modo que ele seja isometricamente isomorfo a $\left(\overline{\mathbb{C}},\|\cdot\|_{\overline{\mathbb{C}}}\right)$.

No que segue, $\operatorname{Re}(z)$ e $\mathcal{I} \mathrm{m}(z)$ denotam a parte real e a parte imaginária de $z$, para todo $z \in \mathbb{C}$.

Proposição 3.1.1 Sejam $\left(z_{\varepsilon}\right)_{\varepsilon}$ e $\left(w_{\varepsilon}\right)_{\varepsilon}$ pertencentes $a \mathbb{C}^{\mathbf{I}}$. Tem-se que:

1. $\left(z_{\varepsilon}\right)_{\varepsilon} \in \mathcal{E}_{M}(\mathbb{C})$ se, e somente se, $\left(\mathcal{R e}\left(z_{\varepsilon}\right)\right)_{\varepsilon} \in \mathcal{E}_{M}(\mathbb{R})$ e $\left(\mathcal{I m}\left(z_{\varepsilon}\right)\right)_{\varepsilon} \in \mathcal{E}_{M}(\mathbb{R})$;

2. $\left(z_{\varepsilon}\right)_{\varepsilon} \in \mathcal{N}(\mathbb{C})$ se, e somente se, $\left(\mathcal{R e}\left(z_{\varepsilon}\right)\right)_{\varepsilon} \in \mathcal{N}(\mathbb{R})$ e $\left(\mathcal{I m}\left(z_{\varepsilon}\right)\right)_{\varepsilon} \in \mathcal{N}(\mathbb{R})$;

3. $\left(z_{\varepsilon}-w_{\varepsilon}\right)_{\varepsilon} \in \mathcal{N}(\mathbb{C})$ se, e somente se, $\left(\mathcal{R e}\left(z_{\varepsilon}\right)-\mathcal{R e}\left(w_{\varepsilon}\right)\right)_{\varepsilon} \in \mathcal{N}(\mathbb{R})$ e $\left(\mathcal{I} \mathrm{m}\left(z_{\varepsilon}\right)-\mathcal{I} \mathrm{m}\left(w_{\varepsilon}\right)\right)_{\varepsilon} \in \mathcal{N}(\mathbb{R})$.

Demonstrą̧ão: Para obtermos 1., basta notarmos que

$$
\max \left\{\left|\mathcal{R e}\left(z_{\varepsilon}\right)\right|,\left|\mathcal{I} \mathrm{m}\left(z_{\varepsilon}\right)\right|\right\} \leq\left|z_{\varepsilon}\right| \leq\left|\mathcal{R e}\left(z_{\varepsilon}\right)\right|+\left|\mathcal{I} \mathrm{m}\left(z_{\varepsilon}\right)\right|, \quad \forall \varepsilon \in \mathbf{I},
$$


e usarmos a definição de elemento moderado.

Para provarmos 2., observemos que, se $b \in \mathbb{R}$, temos que

$$
\lim _{\varepsilon \downarrow 0} \frac{z_{\varepsilon}}{\varepsilon^{b}}=0 \Longleftrightarrow \lim _{\varepsilon \downarrow 0} \frac{\mathcal{R e}\left(z_{\varepsilon}\right)}{\varepsilon^{b}}=0 \text { e } \lim _{\varepsilon \downarrow 0} \frac{\mathcal{I} \operatorname{m}\left(z_{\varepsilon}\right)}{\varepsilon^{b}}=0,
$$

o que nos permite concluir que

$$
\left(z_{\varepsilon}\right)_{\varepsilon} \in \mathcal{N}(\mathbb{C}) \Longleftrightarrow\left(\mathcal{R e}\left(z_{\varepsilon}\right)\right)_{\varepsilon} \in \mathcal{N}(\mathbb{R}) \quad \text { e } \quad\left(\mathcal{I} \mathrm{m}\left(z_{\varepsilon}\right)\right)_{\varepsilon} \in \mathcal{N}(\mathbb{R})
$$

Para 3., é suficiente usarmos 2 . e observarmos que $\operatorname{Re}\left(z_{\varepsilon}-w_{\varepsilon}\right)=\mathcal{R e}\left(z_{\varepsilon}\right)-\mathcal{R e}\left(w_{\varepsilon}\right)$ e $\mathcal{I m}\left(z_{\varepsilon}-w_{\varepsilon}\right)=\operatorname{Im}\left(z_{\varepsilon}\right)-\mathcal{I m}\left(w_{\varepsilon}\right)$, para todo $\varepsilon \in \mathbf{I}$.

Em virtude da Proposição anterior, faz sentido a definição dada a seguir.

Definição 3.1.2 Para cada $z \in \overline{\mathbb{C}}$, chama-se de parte real generalizada de $z$ e parte imaginária generalizada de $z$, e denota-se por $\operatorname{Re}(z)$ e $\operatorname{Im}(z)$, respectivamente, os elementos de $\overline{\mathbb{R}}$ dados por

$$
\operatorname{Re}(z):=\left[\left(\mathcal{R e}\left(z_{\varepsilon}\right)\right)_{\varepsilon}\right]_{\bar{R}} \quad e \quad \operatorname{Im}(z):=\left[\left(\mathcal{I} \mathrm{m}\left(z_{\varepsilon}\right)\right)_{\varepsilon}\right]_{\overline{\boldsymbol{R}}}
$$

onde $\left(z_{\varepsilon}\right)_{\varepsilon}$ é um representante qualquer de $z$.

Observamos que, se $h: \mathbb{C} \hookrightarrow \overline{\mathbb{C}}$ é a função definida acima da Observação 1.1.6, temos que

$$
\operatorname{Re}(h(z))=h(\mathcal{R e}(z)) \quad \text { e } \quad \operatorname{Im}(h(z))=h(\mathcal{I} \operatorname{m}(z)), \forall z \in \mathbb{C}
$$

Proposição 3.1.3 Seja $z \in \overline{\mathbb{C}}$. Então $z=0_{\overline{\mathbb{C}}}$ se, e somente se, $\operatorname{Re}(z)=0_{\bar{R}}$ e $\operatorname{Im}(z)=$ $0_{\bar{R}}$.

Demonstração: Seja $\left(z_{\varepsilon}\right)_{\varepsilon}$ um representante de $z$. Temos que

$$
\begin{aligned}
z=0_{\overline{\mathbb{C}}} \Longleftrightarrow\left(z_{\varepsilon}\right)_{\varepsilon} \in \mathcal{N}(\mathbb{C}) & \Longleftrightarrow\left(\mathcal{R e}\left(z_{\varepsilon}\right)\right)_{\varepsilon} \in \mathcal{N}(\mathbb{R}) \quad \text { e } \quad\left(\mathcal{I m}\left(z_{\varepsilon}\right)\right)_{\varepsilon} \in \mathcal{N}(\mathbb{R}) \\
& \Longleftrightarrow \operatorname{Re}(z)=0_{\bar{R}} \quad \text { e } \quad \operatorname{Im}(z)=0_{\bar{R}},
\end{aligned}
$$


onde a segunda equivalência decorre da Proposição 3.1.1 (2).

De $\mathcal{E}_{M}(\mathbb{R}) \subset \mathcal{E}_{M}(\mathbb{C})$ e $\mathcal{N}(\mathbb{R}) \subset \mathcal{N}(\mathbb{C})$, podemos definir a seguinte aplicação

$$
\begin{aligned}
\psi: \overline{\mathbb{R}} & \longrightarrow \overline{\mathbb{C}} \\
x & \longmapsto\left[\left(x_{\varepsilon}\right)_{\varepsilon}\right]_{\overline{\mathbb{C}}}
\end{aligned},
$$

onde $\left(x_{\varepsilon}\right)_{\varepsilon}$ é um representante qualquer de $x \in \overline{\mathbb{R}}$.

É fácil verificar que $\psi$ tem as seguintes propriedades:

1. $\psi$ é um homorfismo injetor de anéis;

2. $\psi\left(\alpha_{r}^{*}\right)=\alpha_{r}$, para todo $r \in \mathbb{R}$;

3. $x \approx_{\overline{\boldsymbol{R}}} 0_{\overline{\boldsymbol{R}}}$ se, e somente se, $\psi(x) \approx_{\overline{\mathbb{C}}} 0_{\overline{\mathbb{C}}}$, para todo $x \in \overline{\mathbb{R}}$;

4. $\|\psi(x)\|_{\overline{\mathbb{C}}}=\|x\|_{\overline{\mathbf{R}}}$, para todo $x \in \overline{\mathbb{R}}$;

5. $\psi$ é uma imersão isométrica;

6. $\psi(\operatorname{Re}(z))+i \psi(\operatorname{Im}(z))=z$, para todo $z \in \overline{\mathbb{C}}$, onde $i:=\left[(i)_{\varepsilon}\right]_{\overline{\mathbb{C}}}$.

Notamos que a propriedade 1 . de $\psi$ nos permite identificar $\overline{\mathbb{R}} \operatorname{com} \psi(\overline{\mathbb{R}})$ e escrevermos $\overline{\mathbb{R}} \subset \overline{\mathbb{C}}$.

A partir da aplicação $\psi$, construiremos um $\overline{\mathbb{R}}$-isomorfismo entre $\overline{\mathbb{R}}^{2}$ e $\overline{\mathbb{C}}$ (Proposição 3.1.4). Para isso, notamos que $\overline{\mathbb{R}}^{2}$ com as operações

$$
+: \overline{\mathbb{R}}^{2} \times \overline{\mathbb{R}}^{2} \longrightarrow \overline{\mathbb{R}}^{2} \quad \text { e } \quad *: \overline{\mathbb{R}} \times \overline{\mathbb{R}}^{2} \longrightarrow \overline{\mathbb{R}}^{2}
$$

dadas por

$$
\begin{gathered}
(x, y)+(u, v):=(x+u, y+v), \quad \forall(x, y),(u, v) \in \overline{\mathbb{R}}^{2}, \\
\lambda *(x, y):=(\lambda x, \lambda y), \quad \forall \lambda \in \overline{\mathbb{R}} \quad \text { e } \quad \forall(x, y) \in \overline{\mathbb{R}}^{2},
\end{gathered}
$$


e $\overline{\mathbb{C}}$ com a operação usual de soma juntamente com a operação $\cdot: \overline{\mathbb{R}} \times \overline{\mathbb{C}} \longrightarrow \overline{\mathbb{C}}$ definida por

$$
\lambda \cdot z:=\psi(\lambda) z, \forall \lambda \in \overline{\mathbb{R}} \text { e } \forall z \in \overline{\mathbb{C}}
$$

são $\overline{\mathbb{R}}$-módulos.

A fim de simplificarmos a notação, escreveremos $\lambda(x, y)$ e $\lambda z$ para representar $\lambda *(x, y)$ e $\lambda \cdot z$, respectivamente, onde $\lambda \in \overline{\mathbb{R}},(x, y) \in \overline{\mathbb{R}}^{2}$ e $z \in \overline{\mathbb{C}}$.

Proposição 3.1.4 A aplicação $\varphi: \overline{\mathbb{R}}^{2} \longrightarrow \overline{\mathbb{C}}$ dada por $\varphi(x, y):=\psi(x)+i \psi(y)$, para todo $(x, y) \in \overline{\mathbb{R}}^{2}$, onde $\psi$ é definida em (3.1) e $i:=\left[(i)_{\varepsilon}\right]_{\overline{\mathbb{C}}}$, é um $\overline{\mathbb{R}}$-isomorfismo.

Demonstração: Sejam $\lambda \in \overline{\mathbb{R}}$ e $(x, y),(u, v) \in \overline{\mathbb{R}}^{2}$. Então, temos que

$$
\begin{aligned}
\varphi((x, y)+(u, v))=\varphi(x+u, y+v) & =\psi(x+u)+i \psi(y+v) \\
& =\psi(x)+\psi(u)+i(\psi(y)+\psi(v)) \\
& =(\psi(x)+i \psi(y))+(\psi(u)+i \psi(v)) \\
& =\varphi(x, y)+\varphi(u, v) ; \\
\varphi(\lambda(x, y))=\varphi((\lambda x, \lambda y))=\psi(\lambda x)+i \psi(\lambda y) & =\psi(\lambda) \psi(x)+i \psi(\lambda) \psi(y) \\
& =\psi(\lambda)(\psi(x)+i \psi(y)) \\
& =\lambda \varphi(x, y) .
\end{aligned}
$$

Logo, $\varphi$ é um $\overline{\mathbb{R}}$-homomorfismo.

Observemos que, se $\left(x_{\varepsilon}\right)_{\varepsilon}$ e $\left(y_{\varepsilon}\right)_{\varepsilon}$ são representantes de $x$ e $y$, respectivamente, então $\left(x_{\varepsilon}+i y_{\varepsilon}\right)_{\varepsilon}$ é um representante de $\psi(x)+i \psi(y)$. Assim, temos que

$$
\begin{aligned}
(x, y) \in \operatorname{Ker}(\varphi) \Longleftrightarrow \varphi(x, y)=0_{\overline{\mathbb{C}}} & \Longleftrightarrow \psi(x)+i \psi(y)=0_{\overline{\mathbb{C}}} \\
& \Longleftrightarrow\left(x_{\varepsilon}+i y_{\varepsilon}\right)_{\varepsilon} \in \mathcal{N}(\mathbb{C}) \\
& \Longleftrightarrow\left(x_{\varepsilon}\right)_{\varepsilon} \in \mathcal{N}(\mathbb{R}) \quad \text { e } \quad\left(y_{\varepsilon}\right)_{\varepsilon} \in \mathcal{N}(\mathbb{R}) \\
& \Longleftrightarrow(x, y)=(0,0)_{\bar{R}^{2}},
\end{aligned}
$$


onde a penúltima equivalência decorre da Proposição 3.1.1 (2). Portanto, $\varphi$ é injetor.

Seja $z \in \overline{\mathbb{C}}$. Temos que $(\operatorname{Re}(z), \operatorname{Im}(z)) \in \overline{\mathbb{R}}^{2} \mathrm{e}$

$$
\varphi(\operatorname{Re}(z), \operatorname{Im}(z))=\psi(\operatorname{Re}(z))+i \psi(\operatorname{Im}(z))=z
$$

Logo, $\varphi$ é sobrejetor.

Proposição 3.1.5 A aplicação $\varphi$ definida na Proposição 3.1.4 é uma isometria.

Demonstração: Seja $(x, y) \in \overline{\mathbb{R}}^{2}$. Temos, pela Proposição 1.3.7 (1), (2) e (6), que

$$
\begin{aligned}
\|\varphi(x, y)\|_{\overline{\mathbb{C}}}=\|\psi(x)+i \psi(y)\|_{\overline{\mathbb{C}}} & \leq \max \left\{\|\psi(x)\|_{\overline{\mathbb{C}}},\|i\|\left\|_{\overline{\mathbb{C}}}\right\| \psi(y) \|_{\overline{\mathbb{C}}}\right\} \\
& =\max \left\{\|\psi(x)\|_{\overline{\mathbb{C}}},\|\psi(y)\|_{\overline{\mathbb{C}}}\right\} .
\end{aligned}
$$

Observemos que, se $\left(x_{\varepsilon}\right)_{\varepsilon}$ e $\left(y_{\varepsilon}\right)_{\varepsilon}$ são representantes de $x$ e $y$, respectivamente, então, $\left(x_{\varepsilon}\right)_{\varepsilon},\left(y_{\varepsilon}\right)_{\varepsilon}$ e $\left(x_{\varepsilon}+i y_{\varepsilon}\right)_{\varepsilon}$ são representantes de $\psi(x), \psi(y)$ e $\varphi(x, y)$, respectivamente.

Seja $r \in \mathbb{R}$. Então

$$
\lim _{\varepsilon \downarrow 0} \frac{x_{\varepsilon}+i y_{\varepsilon}}{\varepsilon^{r}}=0 \Longleftrightarrow \lim _{\varepsilon \downarrow 0} \frac{x_{\varepsilon}}{\varepsilon^{r}}=0 \quad \text { e } \quad \lim _{\varepsilon \downarrow 0} \frac{y_{\varepsilon}}{\varepsilon^{r}}=0
$$

Logo,

$$
\varphi(x, y) \alpha_{-r} \approx_{\overline{\mathbb{C}}} 0_{\overline{\mathbb{C}}} \Longrightarrow \psi(x) \alpha_{-r} \approx_{\overline{\mathbb{C}}} 0_{\overline{\mathbb{C}}} \quad \text { e } \quad \psi(y) \alpha_{-r} \approx_{\overline{\mathbb{C}}} 0_{\overline{\mathbb{C}}}
$$

e, assim,

$$
\|\varphi(x, y)\|_{\overline{\mathbb{C}}} \geq \max \left\{\|\psi(x)\|_{\overline{\mathbb{C}}},\|\psi(y)\|_{\overline{\mathbb{C}}}\right\}
$$

Portanto, temos que

$$
\|\varphi(x, y)\|_{\overline{\mathbb{C}}}=\max \left\{\|\psi(x)\|_{\overline{\mathbb{C}}},\|\psi(y)\|_{\overline{\mathbb{C}}}\right\}=\max \left\{\|x\|_{\overline{\boldsymbol{R}}},\|y\|_{\overline{\boldsymbol{R}}}\right\}=\|(x, y)\|_{\overline{\boldsymbol{R}}^{2}}
$$

onde a segunda igualdade segue das propriedades de $\psi$ (ver (3.1)).

Dessa forma, pela Proposição 3.1.4, temos que

$$
\left.\|\varphi(x, y)-\varphi(u, v)\|\right|_{\overline{\mathbb{C}}}=\left.\|\varphi(x-u, y-v)\|\right|_{\overline{\mathbb{C}}}=\|(x-u, y-v)\|_{\overline{\boldsymbol{R}}^{2}}=\|(x, y)-(u, v)\|_{\overline{\boldsymbol{R}}^{2}},
$$


para todos $(x, y),(u, v) \in \overline{\mathbb{R}}^{2}$, ou seja, que $\varphi$ é uma isometria.

Convém observarmos que as Proposições 3.1.4 e 3.1.5 nos mostram que, do ponto de vista algébrico - considerados como $\overline{\mathbb{R}}$-módulos - e métrico, não existe diferença entre os conjuntos $\overline{\mathbb{R}}^{2}$ e $\overline{\mathbb{C}}$. Assim, podemos identificar $z \in \overline{\mathbb{C}} \operatorname{com} \varphi^{-1}(z) \in \overline{\mathbb{R}}^{2}$, ou seja, podemos escrever

$$
z=(\operatorname{Re}(z), \operatorname{Im}(z))
$$

No que segue, por simplicidade, sempre que escrevermos $z=(x, y)$, queremos dizer que $x:=\operatorname{Re}(z)$ e $y:=\operatorname{Im}(z)$.

Consideremos o conjunto

$$
\overline{\mathbb{R}}+i \overline{\mathbb{R}}:=\{x+i y: x, y \in \overline{\mathbb{R}}\}, \text { onde } i:=\left[(i)_{\varepsilon}\right]_{\overline{\mathbb{C}}}
$$

e $x+i y=u+i v$ se, e somente se, $x=u$ e $y=v$, para todos $x+i y, u+i v \in \overline{\mathbb{R}}+i \overline{\mathbb{R}}$.

É fácil verificar que $\overline{\mathbb{R}}+i \overline{\mathbb{R}}$ munido com as operações

$$
\begin{gathered}
+:(\overline{\mathbb{R}}+i \overline{\mathbb{R}})^{2} \longrightarrow \overline{\mathbb{R}}+i \overline{\mathbb{R}} ; \\
\cdot: \overline{\mathbb{R}} \times(\overline{\mathbb{R}}+i \overline{\mathbb{R}}) \longrightarrow \overline{\mathbb{R}}+i \overline{\mathbb{R}} ; \\
*: \overline{\mathbb{C}} \times(\overline{\mathbb{R}}+i \overline{\mathbb{R}}) \longrightarrow \overline{\mathbb{R}}+i \overline{\mathbb{R}} ;
\end{gathered}
$$

dadas por

$$
\begin{gathered}
(x+i y)+(u+i v):=(x+u)+i(y+v), \forall x+i y, u+i v \in \overline{\mathbb{R}}+i \overline{\mathbb{R}} ; \\
\lambda \cdot(x+i y)=(\lambda x)+i(\lambda y), \forall \lambda \in \overline{\mathbb{R}} \mathrm{e} \forall x+i y \in \overline{\mathbb{R}}+i \overline{\mathbb{R}} ; \\
\beta *(x+i y)=(\operatorname{Re}(\beta) x-\operatorname{Im}(\beta) y)+i(\operatorname{Re}(\beta) y+\operatorname{Im}(\beta) x), \forall \beta \in \overline{\mathbb{C}} \text { e } \forall x+i y \in \overline{\mathbb{R}}+i \overline{\mathbb{R}} ; \\
\text { é um } \overline{\mathbb{R}} \text {-módulo e um } \overline{\mathbb{C}} \text {-módulo. Além disso, } \overline{\mathbb{R}}+i \overline{\mathbb{R}} \text { munido com a operação + e a } \\
\text { operação } \times:(\overline{\mathbb{R}}+i \overline{\mathbb{R}})^{2} \longrightarrow \overline{\mathbb{R}}+i \overline{\mathbb{R}} \text { dada por } \\
(x+i y) \times(u+i v):=(x u-y v)+i(x v+y u), \forall x+i y, u+i v \in \overline{\mathbb{R}}+i \overline{\mathbb{R}},
\end{gathered}
$$


é um anel, onde o elemento $0+i 0$ é o neutro aditivo e $1+i 0$ é o neutro multiplicativo.

Observamos que, utilizando a operação $\times$, temos

$$
\beta *(x+i y)=(\operatorname{Re}(\beta)+i \operatorname{Im}(\beta)) \times(x+i y), \forall \beta \in \overline{\mathbb{C}} \text { e } \forall x+i y \in \overline{\mathbb{R}}+i \overline{\mathbb{R}} .
$$

A fim de simplificarmos a notação, escreveremos $(x+i y)(u+i v), \lambda(x+i y)$ e $\beta(x+i y)$ para representar $(x+i y) \times(u+i v), \lambda \cdot(x+i y)$ e $\beta *(x+i y)$, respectivamente, onde $(x+i y),(u+i v) \in \overline{\mathbb{R}}+i \overline{\mathbb{R}}, \lambda \in \overline{\mathbb{R}}$ e $\beta \in \overline{\mathbb{C}}$.

Em seguida, mostraremos que $\overline{\mathbb{R}}+i \overline{\mathbb{R}}$, com as operações definidas acima, e $\overline{\mathbb{C}}$ são anéis e módulos isomorfos. Para tanto, utilizaremos o próximo resultado.

\section{Proposição 3.1.6 Sejam}

$$
\begin{aligned}
& \operatorname{Re}: \overline{\mathbb{C}} \rightarrow \overline{\mathbb{R}} \quad \mathrm{Im}: \overline{\mathbb{C}} \rightarrow \overline{\mathbb{R}} \\
& z \longmapsto \operatorname{Re}(z) \quad z \longmapsto \operatorname{Im}(z)
\end{aligned}
$$

onde $\operatorname{Re}(z)$ e $\operatorname{Im}(z)$ são como na Definição 3.1.2. As funções $\operatorname{Re} e \operatorname{Im}$ são $\overline{\mathbb{R}}$-homomorfismos sobrejetores. Além disso, tem-se que

$$
\operatorname{Re}(z w)=\operatorname{Re}(z) \operatorname{Re}(w)-\operatorname{Im}(z) \operatorname{Im}(w) \quad e \quad \operatorname{Im}(z w)=\operatorname{Re}(z) \operatorname{Im}(w)+\operatorname{Im}(z) \operatorname{Re}(w)
$$

para todos $z, w \in \overline{\mathbb{C}}$.

Demonstração: Seja $x=\left[\left(x_{\varepsilon}\right)_{\varepsilon}\right]_{\bar{R}} \in \overline{\mathbb{R}}$. Então $z_{1}:=\left[\left(x_{\varepsilon}\right)_{\varepsilon}\right]_{\overline{\mathbb{C}}} \in \overline{\mathbb{C}}$ e $w_{1}:=\left[\left(i x_{\varepsilon}\right)_{\varepsilon}\right]_{\overline{\mathbb{C}}} \in \overline{\mathbb{C}}$ são tais que

$$
\operatorname{Re}\left(z_{1}\right)=x \quad \text { e } \quad \operatorname{Im}\left(w_{1}\right)=x .
$$

Logo, Re e Im são funções sobrejetoras.

Sejam $\lambda \in \overline{\mathbb{R}}, z, w \in \overline{\mathbb{C}}$ e $\left(\lambda_{\varepsilon}\right)_{\varepsilon},\left(z_{\varepsilon}\right)_{\varepsilon}$ e $\left(w_{\varepsilon}\right)_{\varepsilon}$ representantes de $\lambda, z$ e $w$, respectivamente. 
Para mostrarmos que Re e Im são $\overline{\mathbb{R}}$-homomorfismos, é suficiente notarmos que

$$
\begin{gathered}
\mathcal{R e}\left(z_{\varepsilon}+w_{\varepsilon}\right)=\mathcal{R e}\left(z_{\varepsilon}\right)+\mathcal{R e}\left(w_{\varepsilon}\right) \text { e } \mathcal{I} \mathrm{m}\left(z_{\varepsilon}+w_{\varepsilon}\right)=\mathcal{I} \mathrm{m}\left(z_{\varepsilon}\right)+\mathcal{I} \mathrm{m}\left(w_{\varepsilon}\right), \forall \varepsilon \in \mathbf{I} \\
\mathcal{R e}\left(\lambda_{\varepsilon} z_{\varepsilon}\right)=\lambda_{\varepsilon} \operatorname{Re}\left(z_{\varepsilon}\right) \text { e } \mathcal{I} \operatorname{m}\left(\lambda_{\varepsilon} z_{\varepsilon}\right)=\lambda_{\varepsilon} \mathcal{I} \mathrm{m}\left(z_{\varepsilon}\right), \forall \varepsilon \in \mathbf{I} .
\end{gathered}
$$

Para finalizarmos a prova, basta observarmos que $\mathcal{R e}\left(z_{\varepsilon} w_{\varepsilon}\right)=\mathcal{R e}\left(z_{\varepsilon}\right) \mathcal{R e}\left(w_{\varepsilon}\right)-\mathcal{I} \mathrm{m}\left(z_{\varepsilon}\right) \mathcal{I} \mathrm{m}\left(w_{\varepsilon}\right)$ e $\mathcal{I}_{\mathrm{m}}\left(z_{\varepsilon} w_{\varepsilon}\right)=\mathcal{R e}\left(z_{\varepsilon}\right) \mathcal{I} \mathrm{m}\left(w_{\varepsilon}\right)+\mathcal{I} \mathrm{m}\left(z_{\varepsilon}\right) \mathcal{R e}\left(w_{\varepsilon}\right)$ para todo $\varepsilon \in \mathbf{I}$.

Proposição 3.1.7 Seja $\sigma: \overline{\mathbb{C}} \longrightarrow \overline{\mathbb{R}}+i \overline{\mathbb{R}}$ dada por $\sigma(z):=\operatorname{Re}(z)+i \operatorname{Im}(z)$, para todo $z \in \overline{\mathbb{C}}$, onde $\operatorname{Re}(z)$ e $\operatorname{Im}(z)$ são como na Definição 3.1.2. Tem-se que $\sigma$ é um isomorfismo de anéis e um $\overline{\mathbb{K}}$-isomorfismo, onde $\mathbb{K}=\mathbb{R}$ ou $\mathbb{K}=\mathbb{C}$.

Demonstração: Sejam $\lambda \in \overline{\mathbb{R}}$ e $z, w \in \overline{\mathbb{C}}$. Então temos, pela Proposição 3.1 .6 e por (3.2), que

$$
\begin{aligned}
& \sigma(z+w)=\operatorname{Re}(z+w)+i \operatorname{Im}(z+w)=(\operatorname{Re}(z)+\operatorname{Re}(w))+i(\operatorname{Im}(z)+\operatorname{Im}(w)) \\
& =(\operatorname{Re}(z)+i \operatorname{Im}(z))+(\operatorname{Re}(w)+i \operatorname{Im}(w)) \\
& =\sigma(z)+\sigma(w) \\
& \sigma(z w)=\operatorname{Re}(z w)+i \operatorname{Im}(z w) \\
& =(\operatorname{Re}(z) \operatorname{Re}(w)-\operatorname{Im}(z) \operatorname{Im}(w))+i(\operatorname{Re}(z) \operatorname{Im}(w)+\operatorname{Im}(z) \operatorname{Re}(w)) \\
& =(\operatorname{Re}(z)+i \operatorname{Im}(z))(\operatorname{Re}(w)+i \operatorname{Im}(w)) \\
& =\sigma(z) \sigma(w) \\
& =z * \sigma(w) \\
& \sigma(\lambda z)=\operatorname{Re}(\lambda z)+i \operatorname{Im}(\lambda z)=(\lambda \operatorname{Re}(z))+i(\lambda \operatorname{Im}(z)) \\
& =\lambda(\operatorname{Re}(z)+i \operatorname{Im}(z)) \\
& =\lambda \sigma(z) \text {. }
\end{aligned}
$$


Portanto, $\sigma$ é um homomorfismo de anéis e um $\overline{\mathbb{K}}$-homomorfismo, onde $\mathbb{K}=\mathbb{R}$ ou $\mathbb{I}=\mathbb{C}$.

Temos, pela Proposição 3.1 .3 , que

$$
\begin{aligned}
z \in \operatorname{Ker}(\sigma) \Longleftrightarrow \sigma(z)=0+i 0 & \Longleftrightarrow \operatorname{Re}(z)+i \operatorname{Im}(z)=0+i 0 \\
& \Longleftrightarrow \operatorname{Re}(z)=0_{\overline{\boldsymbol{R}}} \quad \text { e } \operatorname{Im}(z)=0_{\overline{\boldsymbol{R}}} \\
& \Longleftrightarrow z=0_{\overline{\mathbb{C}}} .
\end{aligned}
$$

Portanto, $\sigma$ é injetor.

Sejam $x+i y \in \overline{\mathbb{R}}+i \overline{\mathbb{R}}$ e $\left(x_{\varepsilon}\right)_{\varepsilon},\left(y_{\varepsilon}\right)_{\varepsilon}$ representantes de $x$ e $y$, respectivamente. Então, pela Proposição $3.1 .1(1)$, podemos considerar $z:=\left[\left(x_{\varepsilon}+i y_{\varepsilon}\right)_{\varepsilon}\right]_{\bar{C}}$ e, assim, temos que

$$
\sigma(z)=\operatorname{Re}(z)+i \operatorname{Im}(z)=x+i y
$$

Logo, $\sigma$ é sobrejetor.

Dessa forma, do ponto de vista algébrico, os conjuntos $\overline{\mathbb{R}}+i \overline{\mathbb{R}}$ e $\overline{\mathbb{C}}$ são indistinguíveis. Assim, podemos identificar $z \in \overline{\mathbb{C}} \operatorname{com} \sigma(z) \in \overline{\mathbb{R}}+i \overline{\mathbb{R}}$ e escrever

$$
z=\operatorname{Re}(z)+i \operatorname{Im}(z)
$$

Observamos que o conjunto $\overline{\mathbb{R}}+i \overline{\mathbb{R}}$ será munido com a métrica induzida pela aplicação $\sigma^{-1}$, isto é, se $D$ é a métrica da Definição 1.3 .5 , definimos

$$
\operatorname{dist}(x+i y, u+i v):=D\left(\sigma^{-1}(x+i y), \sigma^{-1}(u+i v)\right)
$$

para todo $x+i y, u+i v \in \bar{R}+i \overline{\mathbb{R}}$, o que implica que $\sigma^{-1}$ é uma isometria. Logo, do ponto de vista métrico, os conjuntos $\overline{\mathbb{R}}+i \overline{\mathbb{R}}$ e $\overline{\mathbb{C}}$ também são indistinguíveis. Notamos, também, que

$$
\sigma^{-1}(x+i y)=\psi(x)+i \psi(y), \forall x+i y \in \overline{\mathbb{R}}+i \overline{\mathbb{R}} .
$$

A fim de simplificarmos a notação, escreveremos $\||(x+i y)-(u+i v)|||$ para indicar $\operatorname{dist}(x+i y, u+i v)$. 
Ressaltamos, ainda, que $\varphi^{-1} \circ \sigma^{-1}: \overline{\mathbb{R}}+i \overline{\mathbb{R}} \longrightarrow \overline{\mathbb{R}}^{2}$ é um $\overline{\mathbb{R}}$-isomorfismo e uma isometria. Portanto, pelo exposto anteriormente, do ponto de vista algébrico - considerados como $\overline{\mathbb{R}}$-módulos - e métrico, não existe diferença entre os conjuntos $\overline{\mathbb{C}}, \overline{\mathbb{R}}^{2}$ e $\overline{\mathbb{R}}+i \overline{\mathbb{R}}$. Assim, se $z \in \overline{\mathbb{C}}$, podemos escrever

$$
z=(x, y)=x+i y
$$

onde $x:=\operatorname{Re}(z)$ e $y:=\operatorname{Im}(z)$. Além disso, como $\varphi$ e $\sigma^{-1}$ são isometrias (Proposição 3.1.5 e (3.3)), para $z=\varphi(x, y)=\sigma^{-1}(x+i y)$, segue que

$$
\|z\|_{\overline{\mathbb{C}}}=\|(x, y)\|_{\bar{R}^{2}}=\|\mid x+i y\| \| .
$$

\subsection{Funções holomorfas e analíticas}

Dentre os resultados aqui encontrados, destacamos a Proposição 3.2 .9 (extraída de [AFJ-1]) e as Proposições 3.2.3 e 3.2.4 (as quais surgiram durante nossos estudos). O primeiro deles nos fornece um resultado sobre série de potências em $\overline{\mathbb{C}}$ e os dois últimos estabelece uma relação entre as condições de Cauchy-Riemann generalizadas e derivabilidade. Esses resultados são análogos aos do contexto clássico.

Em virtude da seção anterior, sempre que escrevermos $z=(x, y)=x+i y$ queremos dizer que $x:=\operatorname{Re}(z)$ e $y:=\operatorname{Im}(z)$, para todo $z \in \overline{\mathbb{C}}$.

Sejam $U$ um subconjunto aberto de $\overline{\mathbb{C}}$ e $f: U \longrightarrow \overline{\mathbb{C}}$. Lembramos que $f$ é derivável em $z_{0} \in U$ (Definição 2.2.2), se existe $w_{0} \in \overline{\mathbb{C}}$ tal que

$$
\lim _{z \rightarrow z_{0}} \frac{f(z)-f\left(z_{0}\right)-w_{0}\left(z-z_{0}\right)}{\alpha_{-\ln \left\|z-z_{0}\right\|}}=0 .
$$

Notamos, ainda, que, pelas Proposições 3.1.4 e 3.1.5, podemos identificar $U$ com o subconjunto aberto $\varphi^{-1}(U)$ de $\overline{\mathbb{R}}^{2}$. Além disso, pela Proposição 3.1 .7 e por (3.3), temos que $\overline{\mathbb{C}}$ é isomorfo e isométrico a $\overline{\mathbb{R}}+i \overline{\mathbb{R}}$. Desse modo, a função $f$ pode ser considerada como uma 
função definida em um subconjunto aberto de $\overline{\mathbb{R}}^{2}$ e a valores em $\overline{\mathbb{R}}+i \overline{\mathbb{R}}$ e, a partir dela, podemos definir as funções $\operatorname{Re}(f), \operatorname{Im}(f): U \subset \overline{\mathbb{R}}^{2} \longrightarrow \overline{\mathbb{R}}$ dadas por

$$
\operatorname{Re}(f)(x, y):=\operatorname{Re}(f(z)) \quad \text { e } \quad \operatorname{Im}(f)(x, y):=\operatorname{Im}(f(z)), \forall z=(x, y) \in U
$$

e escrever

$$
f(z)=f(x, y)=\operatorname{Re}(f)(x, y)+i \operatorname{Im}(f)(x, y), \quad \forall z=(x, y) \in U
$$

As funções $\operatorname{Re}(f)$ e $\operatorname{Im}(f)$ serão chamadas de parte real generalizada de $f$ e de parte imaginária generalizada de $f$, respectivamente.

No que segue, denotaremos $u:=\operatorname{Re}(f)$ e $v:=\operatorname{Im}(f)$ e escreveremos $f=u+i v$.

Proposição 3.2.1 Sejam $U$ um subconjunto aberto de $\overline{\mathbb{C}}$ e $f: U \longrightarrow \overline{\mathbb{C}}$, onde $f:=u+i v$.

Se $z_{0}=\left(x_{0}, y_{0}\right) \in U$ e $w_{0}=u_{0}+i v_{0} \in \overline{\mathbb{R}}+i \overline{\mathbb{R}}$, então são equivalentes:

1. $\lim _{z \rightarrow z_{0}} f(z)=w_{0}$

2. $\lim _{(x, y) \rightarrow\left(x_{0}, y_{0}\right)} u(x, y)=u_{0} \quad e \quad \lim _{(x, y) \rightarrow\left(x_{0}, y_{0}\right)} v(x, y)=v_{0}$.

Demonstração: Suponhamos 1. verdadeira e seja $\rho>0$. Então, por 1., existe $\delta>0$ tal que

$$
z \in U \text { e } 0<\left\|z-z_{0}\right\|_{\overline{\mathbb{C}}}<\delta \Longrightarrow\left\|f(z)-w_{0}\right\|_{\overline{\mathbb{C}}}<\rho .
$$

Portanto, se $z=(x, y) \in U$ e $0<\left\|(x, y)-\left(x_{0}, y_{0}\right)\right\|_{\bar{R}^{2}}<\delta$, temos, por (3.5), que

$$
\begin{aligned}
\max \left\{\left\|u(x, y)-u_{0}\right\|_{\bar{R}},\left\|v(x, y)-v_{0}\right\|_{\bar{R}}\right\} & =\left\|\left(u(x, y)-u_{0}, v(x, y)-v_{0}\right)\right\|_{\overline{\boldsymbol{R}}^{2}} \\
& =\left\|\left(u(x, y)-u_{0}\right)+i\left(v(x, y)-v_{0}\right)\right\| \\
& =\left\|f(z)-w_{0}\right\|_{\overline{\mathbb{C}}} \\
& <\rho .
\end{aligned}
$$

Logo, 2. é verdadeira. 
A seguir, provaremos que 2 . implica 1 ..

Seja $\rho>0$. Então, por 2., existe, $\delta>0$ tal que $(x, y) \in U$ e $0<\left\|(x, y)-\left(x_{0}, y_{0}\right)\right\|_{\overline{\boldsymbol{R}}^{2}}<\delta \Longrightarrow\left\|u(x, y)-u_{0}\right\|_{\overline{\boldsymbol{R}}}<\rho \quad$ e $\quad\left\|v(x, y)-v_{0}\right\|_{\overline{\boldsymbol{R}}}<\rho$ Portanto, se $z=(x, y) \in U$ e $0<\left\|z-z_{0}\right\|_{\overline{\mathbb{C}}}<\delta$, então, por $(3.5)$, temos que

$$
\begin{aligned}
\left\|f(z)-w_{0}\right\|_{\overline{\mathbb{C}}} & =\left\|\left(u(x, y)-u_{0}\right)+i\left(v(x, y)-v_{0}\right)\right\| \\
& =\left\|\left(u(x, y)-u_{0}, v(x, y)-v_{0}\right)\right\|_{\overline{\boldsymbol{R}}^{2}} \\
& =\max \left\{\left\|u(x, y)-u_{0}\right\|_{\overline{\boldsymbol{R}}},\left\|v(x, y)-v_{0}\right\|_{\overline{\boldsymbol{R}}}\right\} \\
& <\rho .
\end{aligned}
$$

Pela Proposição 2.3.5, se uma função $g$ definida em um aberto de $\overline{\mathbb{R}}^{2}$ e a valores em $\overline{\mathbb{R}}$ é diferenciável em um ponto, então existem as derivadas parciais de $g$ nesse ponto. Logo, podemos apresentar a seguinte definição:

Sejam $U$ um subconjunto aberto de $\overline{\mathbb{R}}^{2},\left(x_{0}, y_{0}\right) \in U$ e u,v:U $\longrightarrow \overline{\mathbb{R}}$ diferenciáveis em $\left(x_{0}, y_{0}\right)$. Diz-se que u e $v$ satisfazem as condições de Cauchy-Riemann generalizadas em $\left(x_{0}, y_{0}\right)$ se

$$
\frac{\partial u}{\partial x}\left(x_{0}, y_{0}\right)=\frac{\partial v}{\partial y}\left(x_{0}, y_{0}\right) \quad e \quad \frac{\partial v}{\partial x}\left(x_{0}, y_{0}\right)=-\frac{\partial u}{\partial y}\left(x_{0}, y_{0}\right)
$$

O próximo resultado será útil para provar as Proposições 3.2.3 e 3.2.4, onde se encontram resultados que fornecem relações entre as condições de Cauchy-Riemann generalizadas e a derivabilidade.

Lema 3.2.2 Se $z \in \overline{\mathbb{C}}$ e $r \in \mathbb{R}$, então tem-se que

$$
\operatorname{Re}\left(\frac{z}{\alpha_{-r}}\right)=\frac{\operatorname{Re}(z)}{\alpha_{-r}^{*}} \quad e \quad \operatorname{Im}\left(\frac{z}{\alpha_{-r}}\right)=\frac{\operatorname{Im}(z)}{\alpha_{-r}^{*}}
$$


onde $\alpha_{r}=\left[\left(\varepsilon^{r}\right)_{\varepsilon}\right]_{\overline{\mathbb{C}}} \in \overline{\mathbb{C}}$ e $\alpha_{r}^{*}=\left[\left(\varepsilon^{r}\right)_{\varepsilon}\right]_{\overline{\boldsymbol{R}}} \in \overline{\mathbb{R}}$.

Demonstração: Primeiramente, observemos que

$$
\operatorname{Re}\left(\alpha_{r}\right)=\alpha_{r}^{*} \quad \text { e } \quad \operatorname{Im}\left(\alpha_{r}\right)=0
$$

Então, pela Proposição 3.1.6, temos que

$$
\operatorname{Re}\left(\frac{z}{\alpha_{-r}}\right)=\operatorname{Re}\left(z \alpha_{r}\right)=\operatorname{Re}(z) \operatorname{Re}\left(\alpha_{r}\right)-\operatorname{Im}(z) \operatorname{Im}\left(\alpha_{r}\right)=\operatorname{Re}(z) \alpha_{r}^{*}=\frac{\operatorname{Re}(z)}{\alpha_{-r}^{*}}
$$

$\mathrm{e}$

$$
\operatorname{Im}\left(\frac{z}{\alpha_{-r}}\right)=\operatorname{Im}\left(z \alpha_{r}\right)=\operatorname{Re}(z) \operatorname{Im}\left(\alpha_{r}\right)+\operatorname{Im}(z) \operatorname{Re}\left(\alpha_{r}\right)=\operatorname{Im}(z) \alpha_{r}^{*}=\frac{\operatorname{Im}(z)}{\alpha_{-r}^{*}}
$$

Os próximos dois resultados são análogos aos do contexto clássico.

Proposição 3.2.3 Sejam $U$ um subconjunto aberto de $\overline{\mathbb{C}}$ e $f: U \longrightarrow \overline{\mathbb{C}}$, onde $f=u+i v$. Se $f$ é derivável em $z_{0}=\left(x_{0}, y_{0}\right) \in U$, então u e $v$ são diferenciáveis em $\left(x_{0}, y_{0}\right)$ e satisfazem as condições de Cauchy-Riemann generalizadas em $\left(x_{0}, y_{0}\right)$, isto é,

$$
\frac{\partial u}{\partial x}\left(x_{0}, y_{0}\right)=\frac{\partial v}{\partial y}\left(x_{0}, y_{0}\right) \quad e \quad \frac{\partial v}{\partial x}\left(x_{0}, y_{0}\right)=-\frac{\partial u}{\partial y}\left(x_{0}, y_{0}\right)
$$

Além disso,

$$
f^{\prime}\left(z_{0}\right)=\frac{\partial u}{\partial x}\left(x_{0}, y_{0}\right)+i \frac{\partial v}{\partial x}\left(x_{0}, y_{0}\right)=\frac{\partial v}{\partial y}\left(x_{0}, y_{0}\right)-i \frac{\partial u}{\partial y}\left(x_{0}, y_{0}\right)
$$

Demonstração: Da derivabilidade de $f$ em $z_{0}$, existe $c=c_{1}+i c_{2} \in \overline{\mathbb{C}}$ tal que, se $c:=f^{\prime}\left(z_{0}\right)$ e $h(z):=\frac{E(z)}{\alpha_{-\ln \left\|z-z_{0}\right\|_{\bar{c}}}}$ onde $E(z):=f(z)-f\left(z_{0}\right)-c\left(z-z_{0}\right), \forall z \in U \backslash\left\{z_{0}\right\}$, então

$$
\lim _{z \rightarrow z_{0}} h(z)=0 .
$$


Pelo Lema 3.2.2 e por (3.5), temos que

$$
\begin{aligned}
& \operatorname{Re}(h)(x, y)=\frac{\operatorname{Re}(E(z))}{\alpha_{-\ln \left\|z-z_{0}\right\|_{\overline{\mathbb{C}}}}^{*}}=\frac{u(x, y)-u\left(x_{0}, y_{0}\right)-\left(c_{1}\left(x-x_{0}\right)-c_{2}\left(y-y_{0}\right)\right)}{\alpha_{-\ln \left\|\left(x-x_{0}, y-y_{0}\right)\right\|_{\bar{R}^{2}}}^{*}} \\
& \operatorname{Im}(h)(x, y)=\frac{\operatorname{Im}(E(z))}{\alpha_{-\ln \left\|z-z_{0}\right\|_{\overline{\boldsymbol{C}}}}^{*}}=\frac{v(x, y)-v\left(x_{0}, y_{0}\right)-\left(c_{1}\left(y-y_{0}\right)+c_{2}\left(x-x_{0}\right)\right)}{\alpha_{-\ln \left\|\left(x-x_{0}, y-y_{0}\right)\right\|_{\bar{R}^{2}}^{*}}} .
\end{aligned}
$$

Dessa forma, obtemos, pela Proposição 3.2 .1 e por (3.6), que

$$
\lim _{(x, y) \rightarrow\left(x_{0}, y_{0}\right)} \operatorname{Re}(h)(x, y)=0 \quad \text { e } \quad \lim _{(x, y) \rightarrow\left(x_{0}, y_{0}\right)} \operatorname{Im}(h)(x, y)=0 .
$$

Logo,

$$
\lim _{(x, y) \rightarrow\left(x_{0}, y_{0}\right)} \frac{u(x, y)-u\left(x_{0}, y_{0}\right)-\left(c_{1}\left(x-x_{0}\right)-c_{2}\left(y-y_{0}\right)\right)}{\alpha_{-\ln }^{*}\left\|\left(x-x_{0}, y-y_{0}\right)\right\|_{\overline{\boldsymbol{R}}^{2}}}=0
$$

e

$$
\lim _{(x, y) \rightarrow\left(x_{0}, y_{0}\right)} \frac{v(x, y)-v\left(x_{0}, y_{0}\right)-\left(c_{1}\left(y-y_{0}\right)+c_{2}\left(x-x_{0}\right)\right)}{\alpha_{-\ln }^{*}\left\|\left(x-x_{0}, y-y_{0}\right)\right\|_{\bar{R}^{2}}}=0 .
$$

Portanto, as funções $u$ e $v$ são diferenciáveis em $\left(x_{0}, y_{0}\right)$ e temos, pela Proposição 2.3.5, que

$$
c_{1}=\frac{\partial u}{\partial x}\left(x_{0}, y_{0}\right)=\frac{\partial v}{\partial y}\left(x_{0}, y_{0}\right) \quad \text { e } \quad c_{2}=-\frac{\partial u}{\partial y}\left(x_{0}, y_{0}\right)=\frac{\partial v}{\partial x}\left(x_{0}, y_{0}\right)
$$

Assim,

$$
f^{\prime}\left(z_{0}\right)=\frac{\partial u}{\partial x}\left(x_{0}, y_{0}\right)+i \frac{\partial v}{\partial x}\left(x_{0}, y_{0}\right)=\frac{\partial v}{\partial y}\left(x_{0}, y_{0}\right)-i \frac{\partial u}{\partial y}\left(x_{0}, y_{0}\right)
$$

A seguir, mostraremos que a recíproca da Proposição 3.2 .3 é verdadeira.

Proposição 3.2.4 Sejam $U$ um subconjunto aberto de $\overline{\mathbb{C}}, z_{0}=\left(x_{0}, y_{0}\right) \in U$ e $f: U \longrightarrow \overline{\mathbb{C}}$, onde $f=u+i v$. Se u e v são funções diferenciáveis em $\left(x_{0}, y_{0}\right)$ e satisfazem as condições de Cauchy-Riemann generalizadas em $\left(x_{0}, y_{0}\right)$, então $f$ é derivável em $z_{0}$. 
Demonstração: Sejam $c:=\frac{\partial u}{\partial x}\left(x_{0}, y_{0}\right)+i \frac{\partial v}{\partial x}\left(x_{0}, y_{0}\right) \in \overline{\mathbb{C}}$ e $h: U \backslash\left\{z_{0}\right\} \longrightarrow \overline{\mathbb{C}}$ dada por

$$
h(z):=\frac{E(z)}{\alpha_{-\ln \left\|z-z_{0}\right\|_{\overline{\mathbb{C}}}}}, \text { onde } E(z):=f(z)-f\left(z_{0}\right)-c\left(z-z_{0}\right) .
$$

Vamos mostrar que $\lim _{z \rightarrow z_{0}} h(z)=0$, ou seja, que $f$ é derivável em $z_{0}$.

Pelo Lema 3.2.2 e por (3.5), temos que

$$
\begin{aligned}
& \operatorname{Re}(h)(x, y)=\frac{\operatorname{Re}(E(z))}{\alpha_{-\ln \left\|z-z_{0}\right\|_{\overline{\mathbb{C}}}}^{*}} \\
& =\frac{u(x, y)-u\left(x_{0}, y_{0}\right)-\left(\frac{\partial u}{\partial x}\left(x_{0}, y_{0}\right)\left(x-x_{0}\right)-\frac{\partial v}{\partial x}\left(x_{0}, y_{0}\right)\left(y-y_{0}\right)\right)}{\alpha_{-\ln }^{*}\left\|\left(x-x_{0}, y-y_{0}\right)\right\|_{\bar{R}^{2}}} \\
& =\frac{u(x, y)-u\left(x_{0}, y_{0}\right)-\left(\frac{\partial u}{\partial x}\left(x_{0}, y_{0}\right)\left(x-x_{0}\right)+\frac{\partial u}{\partial y}\left(x_{0}, y_{0}\right)\left(y-y_{0}\right)\right)}{\alpha_{-\ln }^{*}\left\|\left(x-x_{0}, y-y_{0}\right)\right\|_{\overline{\boldsymbol{R}}^{2}}} \\
& \operatorname{Im}(h)(x, y)=\frac{\operatorname{Im}(E(z))}{\alpha_{-\ln \left\|z-z_{0}\right\|_{\overline{\mathbb{C}}}}^{*}} \\
& =\frac{v(x, y)-v\left(x_{0}, y_{0}\right)-\left(\frac{\partial u}{\partial x}\left(x_{0}, y_{0}\right)\left(y-y_{0}\right)+\frac{\partial v}{\partial x}\left(x_{0}, y_{0}\right)\left(x-x_{0}\right)\right)}{\alpha_{-\ln }^{*}\left\|\left(x-x_{0}, y-y_{0}\right)\right\|_{\overline{\boldsymbol{R}}^{2}}} \\
& =\frac{v(x, y)-v\left(x_{0}, y_{0}\right)-\left(\frac{\partial v}{\partial y}\left(x_{0}, y_{0}\right)\left(y-y_{0}\right)+\frac{\partial v}{\partial x}\left(x_{0}, y_{0}\right)\left(x-x_{0}\right)\right)}{\alpha_{-\ln \left\|\left(x-x_{0}, y-y_{0}\right)\right\|_{\overline{\boldsymbol{R}}^{2}}}^{*}}
\end{aligned}
$$

onde a última igualdade em (3.7) e (3.8) segue do fato de que $u$ e $v$ satisfazem as condições de Cauchy-Riemann generalizadas em $\left(x_{0}, y_{0}\right)$. Logo, da diferenciabilidade de $u$ e $v$ em $\left(x_{0}, y_{0}\right)$, temos, pela Proposição 2.3.5, que

$$
\lim _{(x, y) \rightarrow\left(x_{0}, y_{0}\right)} \operatorname{Re}(h)(x, y)=0 \quad \text { e } \quad \lim _{(x, y) \rightarrow\left(x_{0}, y_{0}\right)} \operatorname{Im}(h)(x, y)=0 .
$$

Portanto, obtemos, pela Proposição 3.2.1, que

$$
\lim _{z \rightarrow z_{0}} h(z)=0
$$


o que completa a prova

Definição 3.2.5 Sejam $U$ um subconjunto aberto de $\overline{\mathbb{C}}$ e $f: U \longrightarrow \overline{\mathbb{C}}$. Diz-se que $f$ é holomorfa em $U$, e escreve-se $f \in \mathscr{H}(U)$, se $f$ é derivável em todo ponto de $U$.

Apresentaremos na Proposição 3.2.7 uma caracterização das funções holomorfas que é similar à do contexto clássico. Para esse fim, utilizaremos a seguinte definição:

Definição 3.2.6 Sejam $U$ um subconjunto aberto de $\overline{\mathbb{C}}$ e $f: U \longrightarrow \overline{\mathbb{C}}$, onde $f=u+i v$ e u e v são funções diferenciáveis em todo $(x, y) \in U$. Definem-se as funções $\frac{\partial f}{\partial x}, \frac{\partial f}{\partial y}$, $\frac{\partial f}{\partial \bar{z}}: U \longrightarrow \overline{\mathbb{C}}$ por

$$
\begin{aligned}
\frac{\partial f}{\partial x}(z):=\frac{\partial u}{\partial x}(x, y)+i \frac{\partial v}{\partial x}(x, y), & \forall z=(x, y) \in U \\
\frac{\partial f}{\partial y}(z):=\frac{\partial u}{\partial y}(x, y)+i \frac{\partial v}{\partial y}(x, y), & \forall z=(x, y) \in U \\
\frac{\partial f}{\partial \bar{z}}(z):=\frac{1}{2}\left(\frac{\partial f}{\partial x}(z)+i \frac{\partial f}{\partial y}(z)\right), & \forall z=(x, y) \in U
\end{aligned}
$$

Proposição 3.2.7 Sejam $U$ um subconjunto aberto de $\overline{\mathbb{C}}$ e $f: U \longrightarrow \overline{\mathbb{C}}$, onde $f=u+i v$ e u e v são funções diferenciáveis em todo $(x, y) \in U$. Tem-se que

$$
f \text { é holomorfa em } U \Longleftrightarrow \frac{\partial f}{\partial \bar{z}}(z)=0, \quad \forall z \in U \text {. }
$$

Demonstração: Basta observarmos que, se $z_{0}=\left(x_{0}, y_{0}\right) \in U$, então, pela Proposição 3.1.3, temos que $\frac{\partial f}{\partial \bar{z}}(z)=0$ se, e somente se, $u$ e $v$ satisfazem as condições de Cauchy-Riemann generalizadas em $\left(x_{0}, y_{0}\right)$, e usarmos a Proposição 3.2.3 e a Proposição 3.2.4.

Definição 3.2.8 Sejam $U$ um subconjunto aberto de $\overline{\mathbb{C}}, z_{0} \in U$ e $f: U \longrightarrow \overline{\mathbb{C}}$. Dizse que $f$ é analítica em $z_{0}$ se existe uma seqüência $\left(a_{n}\right)_{n \in N}$ de elementos de $\overline{\mathbb{C}}$ e uma 
série, da forma $\sum_{n=0}^{\infty} a_{n}\left(z-z_{0}\right)^{n}$, que converge em uma vizinhança aberta de $z_{0}$ e tal que $f(z)=\sum_{n=0}^{\infty} a_{n}\left(z-z_{0}\right)^{n}$ nessa vizinhança. Diz-se que $f$ é analítica em $U, \epsilon$ escreve-se $f \in \mathscr{A}(U)$, se $f$ é analítica em todo ponto de $U$.

No que segue, utilizaremos apenas elementos de $\overline{\mathbb{C}}$ e por isso, por praticidade, escreveremos $\|\cdot\|$ no lugar de $\|\cdot\|_{\overline{\mathbb{C}}}$.

Os próximos resultados fornecem algumas propriedades para série de potências em $\overline{\mathbb{C}}$, e serão utilizados para provar que toda função analítica em $U$ é holomorfa em $U$ (Teorema 3.2.11), onde $U$ é um subconjunto aberto de $\overline{\mathbb{C}}$.

Proposição 3.2.9 Sejam $\left(a_{n}\right)_{n \in \mathbb{N}}$ uma seqüência de elementos de $\overline{\mathbb{C}}, z_{0} \in \overline{\mathbb{C}}$ e $r>0$ tais que, para todo $z \in B_{r}\left(z_{0}\right)$, a série $\sum_{n=0}^{\infty} a_{n}\left(z-z_{0}\right)^{n}$ é convergente. Tem-se que:

1. a série $\sum_{n=1}^{\infty} n a_{n}\left(z-z_{0}\right)^{n-1}$ converge uniformemente em $B_{s}\left(z_{0}\right)$, para todo $0<s<r$;

2. se $f: B_{r}\left(z_{0}\right) \longrightarrow \overline{\mathbb{C}}$ é dada por $f(z)=\sum_{n=0}^{\infty} a_{n}\left(z-z_{0}\right)^{n}$, então $f$ é derivável em $B_{r}\left(z_{0}\right) e$

$$
f^{\prime}(z)=\sum_{n=1}^{\infty} n a_{n}\left(z-z_{0}\right)^{n-1}, \quad \forall z \in B_{r}\left(z_{0}\right) .
$$

Demonstração: Sejam $0<s<r$ e $w \in B_{s}\left(z_{0}\right)$. Então, pela Proposição $1.3 .7(2)$, (4) e (5), temos que

$$
\left\|n a_{n}\left(w-z_{0}\right)^{n-1}\right\| \leq\left\|a_{n}\right\|\left\|w-z_{0}\right\|^{n-1} \leq\left\|a_{n}\right\| s^{n-1}=\left\|a_{n}\left(\alpha_{-\ln s}\right)^{n}\right\| s^{-1}, \forall n \geq 1
$$

Observemos que $z_{0}+\alpha_{-\ln s} \in B_{r}\left(z_{0}\right)$, o que implica, por hipótese, que $\sum_{n=0}^{\infty} a_{n}\left(\alpha_{-\ln s}\right)^{n}$ converge e, portanto, pela Proposição 1.4.2, temos que $\lim _{n \rightarrow \infty}\left\|a_{n}\left(\alpha_{-\ln s}\right)^{n}\right\|=0$. Logo,

$$
\lim _{n \rightarrow \infty}\left\|a_{n}\left(\alpha_{-\ln s}\right)^{n}\right\| s^{-1}=0 .
$$


Notemos que, para $1 \leq n<m$,

$$
\begin{aligned}
\left\|\sum_{j=n+1}^{m} j a_{j}\left(w-z_{0}\right)^{j-1}\right\| & \leq \max \left\{\left\|j a_{j}\left(w-z_{0}\right)^{j-1}\right\|: n+1 \leq j \leq m\right\} \\
& \leq \max \left\{\left\|a_{j}\left(\alpha_{-\ln s}\right)^{j}\right\| s^{-1}: n+1 \leq j \leq m\right\},
\end{aligned}
$$

onde a primeira desigualdade segue da Proposição 1.3.7 (1) e a segunda de (3.9). Portanto, por (3.10) e pelo Critério de Cauchy para convergência uniforme ([Rud, Teorema 7.8] juntamente com o fato de $\overline{\mathbb{C}}$ ser espaço métrico completo), concluímos que $\sum_{n=1}^{\infty} n a_{n}\left(w-z_{0}\right)^{n-1}$ converge uniformemente em $B_{s}\left(z_{0}\right)$, o que prova 1 ..

Para 2., consideremos $z \in B_{r}\left(z_{0}\right)$ e $s>0$ tal que $\left\|z-z_{0}\right\|<s<r$. Por 1., podemos definir $g: B_{s}\left(z_{0}\right) \backslash\{z\} \longrightarrow \overline{\mathbb{C}}$ por

$$
\begin{aligned}
g(w) & :=\frac{f(w)-f(z)-(w-z) \sum_{n=1}^{\infty} n a_{n}\left(z-z_{0}\right)^{n-1}}{\alpha_{-\ln \|w-z\|}} \\
& =\frac{\sum_{n=1}^{\infty} a_{n}\left(w-z_{0}\right)^{n}-\sum_{n=1}^{\infty} a_{n}\left(z-z_{0}\right)^{n}-(w-z) \sum_{n=1}^{\infty} n a_{n}\left(z-z_{0}\right)^{n-1}}{\alpha_{-} \ln \|w-z\|} \\
& =\lim _{m \rightarrow \infty} \sum_{n=1}^{m} a_{n} \frac{\left(w-z_{0}\right)^{n}-\left(z-z_{0}\right)^{n}-n\left(z-z_{0}\right)^{n-1}(w-z)}{\alpha_{-} \ln \|w-z\|} .
\end{aligned}
$$

Seja, para cada $m \in \mathbb{N}^{*}$, a função $\varphi_{m}$ definida em $B_{s}\left(z_{0}\right) \backslash\{z\}$ por

$$
\varphi_{m}(w):=\sum_{n=1}^{m} a_{n} \frac{\left(w-z_{0}\right)^{n}-\left(z-z_{0}\right)^{n}-n\left(z-z_{0}\right)^{n-1}(w-z)}{\alpha_{-\ln \|w-z\|}} .
$$

Notemos que, se a seqüência $\left(\varphi_{m}\right)_{m \in N}$ convergir uniformente a $g$ em $B_{s}\left(z_{0}\right) \backslash\{z\}$, então, pelo Teorema de Osgood ([Rud, Teorema 7.11] juntamente com o fato de $\overline{\mathbb{C}}$ ser espaço métrico completo), teremos que

$$
\lim _{w \rightarrow z} g(w)=\lim _{w \rightarrow z} \lim _{m \rightarrow \infty} \varphi_{m}(w)=\lim _{m \rightarrow \infty} \lim _{w \rightarrow z} \varphi_{m}(w)
$$

Observemos, também, que

$$
\lim _{w \rightarrow z} \varphi_{m}(w)=\sum_{n=1}^{m} \lim _{w \rightarrow z} a_{n} \frac{\left(w-z_{0}\right)^{n}-\left(z-z_{0}\right)^{n}-n\left(z-z_{0}\right)^{n-1}(w-z)}{\alpha_{-\ln }\|w-z\|}=0,
$$


para todo $m \in \mathbb{N}^{*}$, pois a função $\left(w-z_{0}\right)^{n}$ é derivável e sua derivada é $n\left(w-z_{0}\right)^{n-1}$, para cada $n \in \mathbb{N}^{*}$ (Exemplo 2.2.4 (3)). Portanto, de (3.11) e (3.12), teremos que $\lim _{w \rightarrow z} g(w)=0$, ou seja, $f^{\prime}(z)=\sum_{n=1}^{\infty} n a_{n}\left(z-z_{0}\right)^{n-1}$. Assim, para concluirmos a prova, resta provarmos que a seqüência $\left(\varphi_{m}\right)_{m \in N}$ converge uniformente a $g$ em $B_{s}\left(z_{0}\right) \backslash\{z\}$.

Fixemos $m \in \mathbb{N}^{*}$. Para todo $w \in B_{s}\left(z_{0}\right) \backslash\{z\}$, temos, pela Proposição 1.3.7 (1), (2), (4) e (8), que

$$
\begin{aligned}
\left\|\varphi_{m}(w)-\varphi_{m-1}(w)\right\| & =\left\|a_{m} \frac{\left(w-z_{0}\right)^{m}-\left(z-z_{0}\right)^{m}-m\left(z-z_{0}\right)^{m-1}(w-z)}{\alpha_{-\ln \|w-z\|}}\right\| \\
& \leq\left\|a_{m}\right\| \max \left\{\frac{\left\|\left(w-z_{0}\right)^{m}-\left(z-z_{0}\right)^{m}\right\|}{\|w-z\|},\left\|z-z_{0}\right\|^{m-1}\right\} .
\end{aligned}
$$

Observemos que

$$
\left(w-z_{0}\right)^{m}=\left((w-z)+\left(z-z_{0}\right)\right)^{m}=\sum_{j=0}^{m}\left(\begin{array}{c}
m \\
j
\end{array}\right)(w-z)^{j}\left(z-z_{0}\right)^{m-j}
$$

e, portanto, pela Proposição $1.3 .7(1),(2)$ e (4), temos que

$$
\begin{aligned}
\left\|\left(w-z_{0}\right)^{m}-\left(z-z_{0}\right)^{m}\right\| & =\left\|\sum_{j=1}^{m}\left(\begin{array}{c}
m \\
j
\end{array}\right)(w-z)^{j}\left(z-z_{0}\right)^{m-j}\right\| \\
& \leq \max \left\{\left\|\left(\begin{array}{c}
m \\
j
\end{array}\right)(w-z)^{j}\left(z-z_{0}\right)^{m-j}\right\|: 1 \leq j \leq m\right\} \\
& \leq \max \left\{\|w-z\|^{j}\left\|z-z_{0}\right\|^{m-j}: 1 \leq j \leq m\right\} .
\end{aligned}
$$

Dessa forma, para todo $w \in B_{s}\left(z_{0}\right) \backslash\{z\}$, obtemos que

$$
\left\|\varphi_{m}(w)-\varphi_{m-1}(w)\right\| \leq\left\|a_{m}\right\| \max \left\{\max \left\{\|w-z\|^{j-1}\left\|z-z_{0}\right\|^{m-j}: 1 \leq j \leq m\right\},\left\|z-z_{0}\right\|^{m-1}\right\} .
$$

Mas, para todo $w \in B_{s}\left(z_{0}\right) \backslash\{z\}$, temos que

$$
\|w-z\|=\left\|\left(w-z_{0}\right)+\left(z_{0}-z\right)\right\| \leq \max \left\{\left\|w-z_{0}\right\|,\left\|z_{0}-z\right\|\right\}<s
$$

e, então, pela Proposição 1.3 .7 (5), temos que

$$
\left\|\varphi_{m}(w)-\varphi_{m-1}(w)\right\| \leq\left\|a_{m}\right\| s^{m-1}=\left\|a_{m}\left(\alpha_{-\ln s}\right)^{m}\right\| s^{-1}, \quad \forall w \in B_{s}\left(z_{0}\right) \backslash\{z\}
$$


Assim, de (3.10), para cada $\sigma>0$, existe $n_{0} \in \mathbb{N}$ tal que, se $m>n_{0}$, então

$$
\left\|\varphi_{m}(w)-\varphi_{m-1}(w)\right\|<\sigma, \quad \forall w \in B_{s}\left(z_{0}\right) \backslash\{z\}
$$

Logo, pela Proposição 1.3.7 (1), para todo $w \in B_{s}\left(z_{0}\right) \backslash\{z\}$ e todo $m>n>n_{0}$, temos que

$$
\left\|\varphi_{m}(w)-\varphi_{n}(w)\right\| \leq \max \left\{\left\|\varphi_{j}(w)-\varphi_{j-1}(w)\right\|: n+1 \leq j \leq m\right\}<\sigma
$$

e, portanto, pelo Critério de Cauchy para convergência uniforme ([Rud, Teorema 7.8] juntamente com o fato de $\overline{\mathbb{C}}$ ser espaço métrico completo), concluímos que $\left(\varphi_{m}\right)_{m \in N}$ converge uniformente a $g$ em $B_{s}\left(z_{0}\right) \backslash\{z\}$.

Corolário 3.2.10 Sejam $\left(a_{n}\right)_{n \in \mathbf{N}}$ uma seqüência de elementos de $\overline{\mathbb{C}}, z_{0} \in \overline{\mathbb{C}}$ e $r>0$ tais que, para todo $z \in B_{r}\left(z_{0}\right)$, a série $\sum_{n=0}^{\infty} a_{n}\left(z-z_{0}\right)^{n}$ é convergente. Se $f: B_{r}\left(z_{0}\right) \longrightarrow \overline{\mathbb{C}}$ é dada por $f(z)=\sum_{n=0}^{\infty} a_{n}\left(z-z_{0}\right)^{n}$, então $f \in \mathscr{C}^{\infty}\left(B_{r}\left(z_{0}\right) ; \overline{\mathbb{C}}\right)$ e, para $k \in \mathbb{N}^{*}$, tem-se

1. $f^{(k)}(z)=\sum_{n=k}^{\infty} \frac{n !}{(n-k) !} a_{n}\left(z-z_{0}\right)^{n-k}$

2. $k ! a_{k}=f^{(k)}\left(z_{0}\right)$.

Demonstração: Para obtermos 1., basta aplicarmos a Proposição 3.2 .9 recursivamente. Para 2., basta usarmos $z=z_{0}$ na expressão $\sum_{n=k}^{\infty} \frac{n !}{(n-k) !} a_{n}\left(z-z_{0}\right)^{n-k}$.

O Teorema a seguir, nos mostra que $\mathscr{A}(U) \subset \mathscr{H}(U)$, onde $U$ é um subconjunto aberto de $\overline{\mathbb{C}}$.

Teorema 3.2.11 Sejam $U$ um subconjunto aberto de $\overline{\mathbb{C}}$ e $f: U \longrightarrow \overline{\mathbb{C}}$. Se $f$ é analítica em $U$, então $f$ é holomorfa em $U$.

Demonstração: Decorre diretamente da Definição 3.2.8 e da Proposição 3.2.9.

Não se sabe, até o momento, se vale a recíproca do Teorema 3.2.11 (que é verdadeira no contexto clássico), isto é, se toda função holomorfa em um subconjunto aberto $U$ 
de $\overline{\mathbb{C}}$ é uma função analítica em $U$. No entanto, na seção 4.2 , definiremos uma imersão $\kappa_{\Omega, \mathbb{C}}: \mathcal{G}(\Omega ; \mathbb{C}) \longrightarrow \overline{\mathbb{C}}^{\tilde{\Omega}_{c}}$ (onde $\Omega$ é um subconjunto aberto de $\mathbb{C}$ ) e veremos que $\kappa_{\Omega, \mathbb{C}}(f)$ é holomorfa em $\widetilde{\Omega}_{c}$ se, e somente se, $\kappa_{\Omega, \mathbb{C}}(f)$ é analítica em $\widetilde{\Omega}_{c}$. 


\section{Capítulo 4}

\section{A álgebra diferencial de Colombeau}

Nas seções 1.5 e 1.6, vimos que as funções generalizadas de Colombeau não são determinadas pelo seu valor pontual mas pelo seu valor pontual generalizado. Esse fato motivou J. Aragona, R. Fernandez e S. O. Juriaans a definirem as imersões $\kappa_{\Omega, \boldsymbol{R}}: \mathcal{G}(\Omega ; \mathbb{R}) \longrightarrow \overline{\mathbb{R}}^{\widetilde{\Omega}_{c}}$ (onde $\Omega$ é um subconjunto aberto de $\mathbb{R}^{n}$ ) e $\kappa_{\Omega, \mathbb{C}}: \mathcal{G}(\Omega ; \mathbb{C}) \longrightarrow \overline{\mathbb{C}}^{\widetilde{\Omega}_{c}}$ (onde $\Omega$ é um subconjunto aberto de $\mathbb{C}$ ). A imagem de cada uma dessas imersões é chamada de álgebra diferencial de Colombeau. Os autores provaram, em [AFJ-1], que alguns resultados verdadeiros no contexto clássico - mas que podem ser falsos no contexto estudado nos capítulos 2 e 3 - também são verdadeiros para a álgebra diferencial de Colombeau. Por exemplo, se $f$ pertence à álgebra diferencial de Colombeau tem-se, no caso em que $\Omega$ é um subconjunto aberto conexo de $\mathbb{R}^{n}$, que sua diferencial é nula se, e somente se, $f$ é constante; tem-se que, no caso em que $\Omega$ é um subconjunto aberto de $\mathbb{C}$, que se $f$ é holomorfa, então $f$ pode ser escrita em série de potências. Esses e outros resultados relativos aos elementos da álgebra diferencial de Colombeau e algumas propriedades das imersões $\kappa_{\Omega, \boldsymbol{R}}$ e $\kappa_{\Omega, \mathbb{C}}$ (extraídas de [AFJ-1]) serão apresentados neste capítulo. Ressaltamos que a partir do estudo do artigo [AFJ-1] nos foi possível, no caso em que $\Omega$ é um subconjunto aberto de $\mathbb{C}$, provar que na álgebra diferencial de Colombeau os conceitos de função holomorfa e de função analítica são equivalentes (Corolário 4.2.11), caracterizar o subconjunto dessa álgebra formado pelas 
funções analíticas (Corolário 4.2.12) e obter a Proposição 4.2.4, a Proposição 4.2.6 e a Proposição 4.2 .8 (afirmações (1), (3) e uma das implicações da afirmação (2)).

No que segue, $\mathcal{G}(\Omega ; \mathbb{K})$ e $\bar{K}$ são munidos com as topologias definidas nas seções $1.7 \mathrm{e}$ 1.3, respectivamente, $\overline{\mathbb{K}}^{n}$ pela topologia produto e $\overline{\mathbb{K}}^{\tilde{\Omega}_{c}}$ com a topologia da convergência pontual, isto é, se $\left(f_{n}\right)_{n \in N}$ é uma seqüência de funções em $\overline{\mathbb{K}}^{\tilde{\Omega}_{c}}$, então $f_{n} \longrightarrow f$ se, e somente se, $f_{n}(x) \longrightarrow f(x)$, para todo $x \in \widetilde{\Omega}_{c}$.

\subsection{A função $\kappa_{\Omega, \boldsymbol{R}}$ e a álgebra $\kappa_{\Omega, \boldsymbol{R}}(\mathcal{G}(\Omega ; \mathbb{R}))$}

O nosso objetivo aqui é apresentar a imersão $\kappa_{\Omega, \boldsymbol{R}}$, onde $\Omega$ é um subconjunto aberto de $\mathbb{R}^{n}$, e estudar algumas propriedades relativas a ela e à álgebra $\kappa_{\Omega, \boldsymbol{R}}(\mathcal{G}(\Omega ; \mathbb{R}))$. Entre os resultados abordados, destacamos que $\kappa_{\Omega, R}(\mathcal{G}(\Omega ; \mathbb{R})) \subset \mathscr{C}^{\infty}\left(\tilde{\Omega}_{c} ; \overline{\mathbb{R}}\right)$, que as funções pertencentes a $\kappa_{\Omega, \boldsymbol{R}}(\mathcal{G}(\Omega ; \mathbb{R}))$ são diferenciáveis e, além disso, que para essas funções vale um resultado similar ao Teorema do Valor Médio do contexto clássico (Teorema 4.1.6). Finalizamos a seção com algumas propriedades dos elementos de $\kappa_{\Omega, R}\left(\mathscr{C}^{\infty}(\Omega ; \mathbb{R})\right)$ (Proposição 4.1.12 e Teorema 4.1.13).

Nesta seção, utilizaremos as notações da seção 2.3, a menos de menção em contrário.

Definição 4.1.1 Seja $\Omega$ um subconjunto aberto de $\mathbb{R}^{n}$. Define-se

$$
\begin{aligned}
\kappa_{\Omega, \boldsymbol{R}}: \mathcal{G}(\Omega ; \mathbb{R}) & \longrightarrow \overline{\mathbb{R}}^{\tilde{\Omega}_{c}} \\
f & \longmapsto \kappa_{\Omega, \boldsymbol{R}}(f)
\end{aligned},
$$

onde $\kappa_{\Omega, \boldsymbol{R}}(f)(x):=f(x)$, para todo $x \in \widetilde{\Omega}_{c}$, e $f(x)$ é o valor pontual generalizado de $f$ em $x$ definido na Definição 1.6.5.

A fim de facilitarmos a escrita, escreveremos, a menos de menção em contrário, $\kappa$ ao invés de $\kappa_{\Omega, R}$. 
O resultado a seguir nos fornece algumas propriedades da função $\kappa$.

Proposição 4.1.2 Seja $\Omega$ um subconjunto aberto de $\mathbb{R}^{n}$. A função $\kappa: \mathcal{G}(\Omega ; \mathbb{R}) \longrightarrow \overline{\mathbb{R}}^{\tilde{\Omega}_{c}}$, definida na Definição 4.1.1, é um homomorfismo injetor de $\overline{\mathbb{R}}$-álgebras. Além disso, $\kappa$ é contínua.

Demonstração: É fácil verificar que $\kappa$ é um homomorfismo de $\overline{\mathbb{R}}$-álgebras. Desse modo, temos que $\kappa$ é injetora se, e somente se, $\operatorname{Ker} \kappa=\{0\}$. Mas,

$f \in \operatorname{Ker} \kappa \Longleftrightarrow \kappa(f)=0 \Longleftrightarrow \kappa(f)(x)=0, \forall x \in \widetilde{\Omega}_{c} \Longleftrightarrow f(x)=0, \forall x \in \widetilde{\Omega}_{c} \Longleftrightarrow f=0$,

onde a última equivalência segue do Teorema 1.6.6. Portanto, $\kappa$ é injetora.

A seguir, vamos mostrar que $\kappa$ é contínua.

Primeiramente, observemos que é suficiente provarmos que $\kappa$ é contínua no ponto zero, pois $\kappa$ é homomorfismo. Para isso, notemos que, como $\mathcal{G}(\Omega ; \mathbb{R})$ é espaço métrico (e, portanto, espaço E1) e $\overline{\mathbb{R}}^{\widetilde{\Omega}_{c}}$ é espaço de Hausdorff-([Eng, Proposição 2.6.4]), basta mostrarmos que, se $\left(f_{j}\right)_{j \in N}$ é uma seqüência qualquer de elementos de $\mathcal{G}(\Omega ; \mathbb{R})$ tal que $\lim _{j \rightarrow \infty} f_{j}=0$, então $\lim _{j \rightarrow \infty} \kappa\left(f_{j}\right)=0$, ([Lim, Proposição $5^{*}$, pg 125]), ou seja, que

$$
\lim _{j \rightarrow \infty} \kappa\left(f_{j}\right)(x)=0, \forall x \in \widetilde{\Omega}_{c} .
$$

Sejam $\left(f_{j}\right)_{j \in N}$ uma seqüência de elementos de $\mathcal{G}(\Omega ; \mathbb{R})$ tal que $\lim _{j \rightarrow \infty} f_{j}=0$ e $x=\left(\left[\left(x_{1 \varepsilon}\right)_{\varepsilon}\right], \ldots,\left[\left(x_{n \varepsilon}\right)_{\varepsilon}\right]\right) \in \widetilde{\Omega}_{c}$, onde $\left(x_{\varepsilon}\right)_{\varepsilon}:=\left(\left(x_{1 \varepsilon}, \ldots, x_{n \varepsilon}\right)\right)_{\varepsilon} \in \Omega_{c}$. Então existem $K \subset \subset \Omega$ e $\eta \in \mathbf{I}$ tais que $x_{\varepsilon} \in K$, para todo $\varepsilon \in \mathbf{I}_{\eta}$.

Seja $\left(\Omega_{m}\right)_{m \in N}$ uma seqüencia exaustiva de abertos de $\Omega$ e fixemos $m_{0} \in \mathbb{N}$ tal que $K \subset \Omega_{m_{0}}$.

Provaremos que

$$
\forall p \in \mathbb{N}, \exists N_{p} \in \mathbb{N} \text { tal que } f_{j}(x) \alpha_{-p} \approx 0, \forall j \geq N_{p}
$$

e, assim, pela Proposição 1.4.3, segue que $\lim _{j \rightarrow \infty} \kappa\left(f_{j}\right)(x)=0$. 
Seja $p \in \mathbb{N}$. Como $\lim _{j \rightarrow \infty} f_{j}=0$, temos, substituindo $m$ e $q$ da Proposição 1.7 .11 por $m_{0}$ e $p$, respectivamente, que existe $N_{p} \in \mathbb{N}$ tal que $\alpha_{-p}\left|f_{j}\right|_{0, m_{0}} \approx 0$, para todo $j \geq N_{p}$. Assim, como $\left|f_{j}(x)\right| \leq\left.|| f_{j}\right|_{0, m_{0}} \mid$, para todo $j \in \mathbb{N}$ (pois $K \subset \Omega_{m_{0}}$ ) temos, pela Proposição $1.2 .9(2)$ e (4), que $f_{j}(x) \alpha_{-p} \approx 0$, para todo $j \geq N_{p}$, o que prova (4.1).

Da Proposição 4.1.2 temos que $\kappa(\mathcal{G}(\Omega ; \mathbb{R}))$ é uma $\overline{\mathbb{R}}$-álgebra, onde $\Omega$ é um subconjunto aberto de $\mathbb{R}^{n}$.

Por comodidade, no que segue, denotaremos

$$
\frac{\partial}{\partial x_{i}}:=\partial_{i} \quad \text { e } \quad \frac{\partial^{2}}{\partial x_{i}^{2}}:=\partial_{i}^{2}, \quad \forall 1 \leq i \leq n,
$$

onde $x_{i}$ denota a i-ésima coordenada de um elemento qualquer $x$ de $\overline{\mathbb{R}}^{n}$ ou de $\mathbb{R}^{n}$.

Nos próximos resultados, apresentaremos algumas propriedades dos elementos de $\kappa(\mathcal{G}(\Omega ; \mathbb{R}))$.

Proposição 4.1.3 Seja $\Omega$ um subconjunto aberto de $\mathbb{R}^{n}$. Então $\kappa(\mathcal{G}(\Omega ; \mathbb{R})) \subset \mathscr{C}\left(\widetilde{\Omega}_{c} ; \overline{\mathbb{R}}\right)$.

Demonstração: Seja $f \in \mathcal{G}(\Omega ; \mathbb{R})$. Provaremos que $\lim _{y \rightarrow x}(\kappa(f)(y)-\kappa(f)(x))=0$, para todo $x \in \widetilde{\Omega}_{c}$.

Seja $x:=\left(x_{1}, \ldots, x_{n}\right)=\left(\left[\left(x_{1 \varepsilon}\right)_{\varepsilon}\right], \ldots,\left[\left(x_{n \varepsilon}\right)_{\varepsilon}\right]\right) \in \widetilde{\Omega}_{c}$, onde $\left(x_{\varepsilon}\right)_{\varepsilon}:=\left(\left(x_{1 \varepsilon}, \ldots, x_{n \varepsilon}\right)\right)_{\varepsilon} \in \Omega_{c}$.

Consideremos $y \in B_{1}(x) \subset \widetilde{\Omega}_{c}$ (Proposição $1.6 .7(1)$ ). Então $y=\left(y_{1}, \ldots, y_{n}\right):=$ $\left(\left[\left(y_{1 \varepsilon}\right)_{\varepsilon}\right], \ldots,\left[\left(y_{n \varepsilon}\right)_{\varepsilon}\right]\right) \in \tilde{\Omega}_{c}$, onde $\left(y_{\varepsilon}\right)_{\varepsilon}:=\left(\left(y_{1 \varepsilon}, \ldots, y_{n \varepsilon}\right)\right)_{\varepsilon} \in \Omega_{c}$. Como $\|x-y\|<1$, temos, pela Proposição 1.4 .5 (1) (a), que $x \approx y$, o que implica por (1.2) que

$$
\lim _{\varepsilon \downarrow 0}\left|x_{\varepsilon}-y_{\varepsilon}\right|=0
$$

Logo, pela Proposição 1.6.4 (1), existem $K_{1} \subset \subset \Omega$ e $\eta_{1} \in \mathbf{I}$ tais que $P_{\varepsilon} \subset K_{1}$, para todo $\varepsilon \in \mathbf{I}_{\eta_{1}}$, onde $P_{\varepsilon}$ é o segmento de extremidades $x_{\varepsilon}$ e $y_{\varepsilon}$. 
Seja $\left(f_{\varepsilon}\right)_{\varepsilon}$ um representante de $f$. Notemos que $f_{\varepsilon}$ é diferenciável, para todo $\varepsilon \in \mathbf{I}$, pois $\left(f_{\varepsilon}\right)_{\varepsilon} \in\left(\mathscr{C}^{\infty}(\Omega ; \mathbb{R})\right)^{\mathbf{I}}$. Portanto, para cada $\varepsilon \in \mathbf{I}_{\eta_{1}}$ fixado, temos, pelo Teorema do Valor Médio, que existe $z_{\varepsilon} \in P_{\varepsilon}$ tal que

$$
f_{\varepsilon}\left(x_{\varepsilon}\right)-f_{\varepsilon}\left(y_{\varepsilon}\right)=\sum_{i=1}^{n} \partial_{i} f_{\varepsilon}\left(z_{\varepsilon}\right)\left(x_{i \varepsilon}-y_{i \varepsilon}\right)
$$

Fixemos $1 \leq i \leq n$. Como $\left(f_{\varepsilon}\right)_{\varepsilon} \in \mathcal{E}_{M}[\Omega ; \mathbb{R}]$, existem $N_{i} \in \mathbb{N}, c_{i}>0$ e $\nu_{i} \in \mathbf{I}_{\eta_{1}}$ tais que

$$
\left\|\partial_{i} f_{\varepsilon}(\cdot)\right\|_{K_{1}} \leq c_{i} \varepsilon^{-N_{i}}, \forall \varepsilon \in \mathbf{I}_{\nu_{i}} .
$$

Consideremos $c:=\max \left\{c_{i}: 1 \leq i \leq n\right\}, N:=\max \left\{N_{i}: 1 \leq i \leq n\right\}$ e $\eta:=\min \left\{\nu_{i}:\right.$ $1 \leq i \leq n\}$. Então, por (4.2) e (4.3), temos que

$$
\left|f_{\varepsilon}\left(x_{\varepsilon}\right)-f_{\varepsilon}\left(y_{\varepsilon}\right)\right| \leq \sum_{i=1}^{n} c \varepsilon^{-N}\left|x_{i \varepsilon}-y_{i \varepsilon}\right|=\left|\sum_{i=1}^{n} c \varepsilon^{-N}\right| x_{i \varepsilon}-y_{i \varepsilon}||, \forall \varepsilon \in \mathbf{I}_{\eta} .
$$

Logo,

$$
|\kappa(f)(x)-\kappa(f)(y)| \leq\left|\sum_{i=1}^{n} c \alpha_{-N}\right| x_{i}-y_{i}||
$$

e, assim, pela Proposição 1.3.7 (1), (4), (5), (9) e (10), temos que

$$
\begin{aligned}
\|\kappa(f)(x)-\kappa(f)(y)\| \leq\left\|c \alpha_{-N} \sum_{i=1}^{n}\left|x_{i}-y_{i}\right|\right\| & \leq e^{N}\left\|\sum_{i=1}^{n}\left|x_{i}-y_{i}\right|\right\| \\
& \leq e^{N} \max \left\{\left\|x_{i}-y_{i}\right\|: 1 \leq i \leq n\right\} \\
& =e^{N}\|x-y\| .
\end{aligned}
$$

Portanto,

$$
\|\kappa(f)(x)-\kappa(f)(y)\| \leq e^{N}\|x-y\|, \forall y \in B_{1}(x),
$$

e, como $\|\kappa(f)(y)-\kappa(f)(x)\|=\|\kappa(f)(x)-\kappa(f)(y)\|$ (Proposição 1.3.7 (4)), concluímos que

$$
\lim _{y \rightarrow x}(\kappa(f)(y)-\kappa(f)(x))=0 .
$$

Proposição 4.1.4 Seja $\Omega$ um subconjunto aberto de $\mathbb{R}^{n}$. Se $f \in \mathcal{G}(\Omega ; \mathbb{R})$, então

$$
\frac{\partial(\kappa(f))}{\partial x_{i}}=\kappa\left(\frac{\partial f}{\partial x_{i}}\right)
$$

para todo $1 \leq i \leq n$. 
Demonstração: Seja $\left\{e_{i}: 1 \leq i \leq n\right\}$ a base canônica de $\overline{\mathbb{R}}^{n}$, isto é,

$$
e_{i}:=\left[\left(\mu_{i}\right)_{\varepsilon}\right], \forall 1 \leq i \leq n
$$

onde $\mu_{i}$ é o i-ésimo elemento da base canônica de $\mathbb{R}^{n}$.

É suficiente provarmos que, para cada $1 \leq i \leq n$ fixado, tem-se

$$
\lim _{h \rightarrow 0} \frac{\left\|\kappa(f)\left(c+h e_{i}\right)-\kappa(f)(c)-\kappa\left(\partial_{i} f\right)(c) h\right\|}{\|h\|}=0, \forall c \in \widetilde{\Omega}_{c}
$$

pois, pela Proposição 1.3.7 (5) e pela Definição 2.3.2, seguirá que $\partial_{i}(\kappa(f))=\kappa\left(\partial_{i} f\right)$.

Fixemos $1 \leq i \leq n$ e seja $c=\left(\left[\left(c_{1 \varepsilon}\right)_{\varepsilon}\right], \ldots,\left[\left(c_{n \varepsilon}\right)_{\varepsilon}\right]\right) \in \widetilde{\Omega}_{c}$, onde $\left(c_{\varepsilon}\right)_{\varepsilon}:=\left(\left(c_{1 \varepsilon}, \ldots, c_{n \varepsilon}\right)\right)_{\varepsilon} \in$ $\Omega_{c}$.

Consideremos $h:=\left[\left(h_{\varepsilon}\right)_{\varepsilon}\right] \in \overline{\mathbb{R}} \operatorname{com} 0<\|h\|<1$ e seja $\left(y_{\varepsilon}\right)_{\varepsilon}:=\left(c_{\varepsilon}+h_{\varepsilon} \mu_{i}\right)_{\varepsilon}$. De $\|h\|<1$, temos, pela Proposição 1.4 .5 (1) (a), que $h \approx 0$. Portanto, obtemos que

$$
\lim _{\varepsilon \downarrow 0}\left|y_{\varepsilon}-c_{\varepsilon}\right|=\lim _{\varepsilon \downarrow 0}\left|c_{\varepsilon}+h_{\varepsilon} \mu_{i}-c_{\varepsilon}\right|=\lim _{\varepsilon \downarrow 0}\left|h_{\varepsilon}\right|=0
$$

Logo, pela Proposição 1.6.4 (1), existem $K_{1} \subset \subset \Omega$ e $\eta_{1} \in \mathbf{I}$ tais que $P_{\varepsilon} \subset K_{1}$, para todo $\varepsilon \in \mathbf{I}_{\eta_{1}}$, onde $P_{\varepsilon}$ é o segmento de extremidades $c_{\varepsilon}$ e $c_{\varepsilon}+h_{\varepsilon} \mu_{i}$.

Seja $\left(f_{\varepsilon}\right)_{\varepsilon}$ um representante de $f$. Notemos que $f_{\varepsilon} \in \mathscr{C}^{\infty}(\Omega ; \mathbb{R})$, para todo $\varepsilon \in \mathbf{I}$. Assim, para cada $\varepsilon \in \mathbf{I}_{\eta_{1}}$ fixado, temos, pela Fórmula de Taylor, que existe $z_{\varepsilon} \in P_{\varepsilon}$ tal que

$$
f_{\varepsilon}\left(c_{\varepsilon}+h_{\varepsilon} \mu_{i}\right)=f_{\varepsilon}\left(c_{\varepsilon}\right)+\partial_{i} f_{\varepsilon}\left(c_{\varepsilon}\right) h_{\varepsilon}+\frac{1}{2} \partial_{i}^{2} f_{\varepsilon}\left(\bar{z}_{\varepsilon}\right) h_{\varepsilon}^{2}
$$

Como $\left(f_{\varepsilon}\right)_{\varepsilon} \in \mathcal{E}_{M}[\Omega ; \mathbb{R}]$ e $K_{1} \subset \subset \Omega$, existem $N_{i} \in \mathbb{N}, c_{i}>0$ e $\nu_{i} \in \mathbf{I}_{\eta_{1}}$ tais que

$$
\left|\partial_{i}^{2} f_{\varepsilon}\left(z_{\varepsilon}\right)\right| \leq c_{i} \varepsilon^{-N_{i}}, \forall \varepsilon \in \mathbf{I}_{\nu_{i}}
$$

Então, temos que

$$
\left|f_{\varepsilon}\left(c_{\varepsilon}+h_{\varepsilon} \mu_{i}\right)-f_{\varepsilon}\left(c_{\varepsilon}\right)-\partial_{i} f_{\varepsilon}\left(c_{\varepsilon}\right) h_{\varepsilon}\right| \leq \frac{1}{2} c_{i} \varepsilon^{-N_{i}} h_{\varepsilon}^{2}=\left|\frac{1}{2} c_{i} \varepsilon^{-N_{i}} h_{\varepsilon}^{2}\right|, \forall \varepsilon \in \mathbf{I}_{\nu_{i}} .
$$

Logo, como $c+h e_{i} \in B_{1}(c) \subset \widetilde{\Omega}_{c}$ (Proposição 1.6.7 (1)), obtemos que

$$
\left|\kappa(f)\left(c+h e_{i}\right)-\kappa(f)(c)-\kappa\left(\partial_{i} f\right)(c) h\right| \leq\left|\frac{1}{2} c_{i} \alpha_{-N_{i}} h^{2}\right|
$$


e, portanto, pela Proposição 1.3.7 (2), (4), (5) e (10), temos que

$$
\left\|\kappa(f)\left(c+h e_{i}\right)-\kappa(f)(c)-\kappa\left(\partial_{i} f\right)(c) h\right\| \leq\left\|\frac{1}{2} c_{i} \alpha_{-N_{i}} h^{2}\right\|=e^{N_{i}}\left\|h^{2}\right\| \leq e^{N_{i}}\|h\|^{2} .
$$

Assim,

$$
\frac{\left\|\kappa(f)\left(c+h e_{i}\right)-\kappa(f)(c)-\kappa\left(\partial_{i} f\right)(c) h\right\|}{\|h\|} \leq e^{N_{i}}\|h\|
$$

Portanto,

$$
\lim _{h \rightarrow 0} \frac{\left\|\kappa(f)\left(c+h e_{i}\right)-\kappa(f)(c)-\kappa\left(\partial_{i} f\right)(c) h\right\|}{\|h\|}=0 .
$$

Se $f$ é uma função de uma variável, também escreveremos $f^{\prime}$ para denotar a derivada de $f$. Notamos que, se $n=1$, então, da Proposição anterior, temos que

$$
(\kappa(f))^{\prime}=\kappa\left(f^{\prime}\right), \forall f \in \mathcal{G}(\Omega ; \mathbb{R})
$$

Da Proposição 4.1.4, temos que:

Se $\Omega$ é um subconjunto aberto de $\mathbb{R}^{n}$ e $f \in \mathcal{G}(\Omega ; \mathbb{R})$, então

$$
\frac{\partial^{2} \kappa(f)}{\partial x_{i} \partial x_{j}}=\frac{\partial^{2} \kappa(f)}{\partial x_{j} \partial x_{i}}, \forall 1 \leq i, j \leq n,
$$

pois

$$
\begin{aligned}
\frac{\partial^{2} \kappa(f)}{\partial x_{i} \partial x_{j}}=\frac{\partial}{\partial x_{i}}\left(\frac{\partial \kappa(f)}{\partial x_{j}}\right)=\frac{\partial}{\partial x_{i}}\left(\kappa\left(\frac{\partial f}{\partial x_{j}}\right)\right)=\kappa\left(\frac{\partial}{\partial x_{i}}\left(\frac{\partial f}{\partial x_{j}}\right)\right) & =\kappa\left(\frac{\partial}{\partial x_{j}}\left(\frac{\partial f}{\partial x_{i}}\right)\right) \\
& =\frac{\partial}{\partial x_{j}}\left(\kappa\left(\frac{\partial f}{\partial x_{i}}\right)\right) \\
& =\frac{\partial}{\partial x_{j}}\left(\frac{\partial \kappa(f)}{\partial x_{i}}\right) \\
& =\frac{\partial^{2} \kappa(f)}{\partial x_{j} \partial x_{i}} .
\end{aligned}
$$

Proposição 4.1.5 Seja $\Omega$ um subconjunto aberto de $\mathbb{R}^{n}$. Tem-se que: 


$$
\begin{aligned}
& \text { 1. } \kappa(\mathcal{G}(\Omega ; \mathbb{R})) \subset \mathscr{C}^{\infty}\left(\widetilde{\Omega}_{c} ; \overline{\mathbb{R}}\right) ; \\
& \text { 2. } \kappa(\mathcal{G}(\Omega ; \mathbb{R})) \neq \mathscr{C}^{\infty}\left(\widetilde{\Omega}_{c} ; \overline{\mathbb{R}}\right) \text {. }
\end{aligned}
$$

Demonstração: Seja $f \in \mathcal{G}(\Omega ; \mathbb{R})$. Observemos que, se $\beta=\left(\beta_{1}, \ldots, \beta_{n}\right) \in \mathbb{N}^{n}$ e $i=\min \{j$ : $\left.\beta_{j} \neq 0\right\}$, então existe $\gamma \in \mathbb{N}^{n}$ tal que

$$
\partial^{\beta} f=\frac{\partial}{\partial x_{i}}\left(\partial^{\gamma} f\right) \quad \text { e } \quad \partial^{\gamma} f \in \mathcal{G}(\Omega ; \mathbb{R}) .
$$

Desse fato e da Proposição 4.1.4, é claro que

$$
\partial^{\alpha}(\kappa(f))=\kappa\left(\partial^{\alpha} f\right), \forall \alpha \in \mathbb{N}^{n}
$$

Assim, pela Proposição 4.1.3, temos que $\partial^{\alpha}(\kappa(f))$ é contínua, para todo $\alpha \in \mathbb{N}^{n}$, o que prova $1 .$.

Para provarmos 2., consideremos $f: \overline{\mathbb{R}} \longrightarrow \overline{\mathbb{R}}$ dada por

$$
f(x):=\left\{\begin{array}{ll}
\alpha_{-2 \ln \|x\|} & , \text { se } x \neq 0 \\
0 & , \text { se } x=0
\end{array} .\right.
$$

Suponhamos que exista $g \in \mathcal{G}(\mathbb{R} ; \mathbb{R})$ tal que $\kappa(g)=f$. Logo, por (4.4) e pelo Exemplo $2.2 .4(2)$, temos que

$$
\kappa\left(g^{\prime}\right)=(\kappa(g))^{\prime}=f^{\prime}=0
$$

e, como $\kappa$ é injetora (Proposição 4.1.2), segue que $g^{\prime}=0$. Desse modo, como $\mathbb{R}$ é conexo, obtemos, pela Proposição 1.5.12, que $g$ é constante. Assim, pela Definição 1.5.8, existe $\lambda \in \overline{\mathbb{R}}$ tal que $g=\left[\left(\lambda_{\varepsilon}^{*}\right)_{\varepsilon}\right]$, onde $\left(\lambda_{\varepsilon}\right)_{\varepsilon}$ é um representante de $\lambda$ e $\lambda_{\varepsilon}^{*}(x)=\lambda_{\varepsilon}$, para todo $\varepsilon \in \mathbf{I}$ e todo $x \in \mathbb{R}$. Portanto, $\kappa(g)(y)=g(y)=\left[\left(\lambda_{\varepsilon}^{*}\left(y_{\varepsilon}\right)\right)_{\varepsilon}\right]=\left[\left(\lambda_{\varepsilon}\right)_{\varepsilon}\right]=\lambda$, para todo $y \in \widetilde{\mathbb{R}}_{c}$, onde $\left(y_{\varepsilon}\right)_{\varepsilon} \in \mathbb{R}_{c}$ e $y=\left[\left(y_{\varepsilon}\right)_{\varepsilon}\right]$, ou seja, $f=\kappa(g)$ é constante, o que é um absurdo.

Nos próximos resultados, utilizaremos a seguinte definição:

Sejam $\Omega$ um subconjunto aberto de $\mathbb{R}^{n}$ e $K \subset \subset \Omega$. Diz-se que $x \in \overline{\mathbb{R}}^{n}$ é um elemento de $\widetilde{K}$ se, e somente se, existe $\left(x_{\varepsilon}\right)_{\varepsilon}:=\left(\left(x_{1 \varepsilon}, \ldots, x_{n \varepsilon}\right)\right)_{\varepsilon} \in \Omega_{c}$ tal que $x=\left(\left[\left(x_{1 \varepsilon}\right)_{\varepsilon}\right], \ldots,\left[\left(x_{n \varepsilon}\right)_{\varepsilon}\right]\right)$ e existe $\eta \in \mathbf{I}$ tal que $x_{\varepsilon} \in K$, para todo $\varepsilon \in \mathbf{I}_{\eta}$. 
Observamos que $\widetilde{K} \subset \widetilde{\Omega}_{c}$ e que, se $x \in \widetilde{\Omega}_{c}$, então, da definição de $\widetilde{\Omega}_{c}$, existe $L \subset \subset \Omega$ tal que $x \in \tilde{L}$.

No capítulo 2, vimos que, para funções definidas em um subconjunto aberto de $\overline{\mathbb{R}}$ e a valores em $\mathbb{R}$, o Teorema do Valor Médio é falso em geral (Observação 2.2.5 (b)). No entanto, para funções pertencentes a $\kappa(\mathcal{G}(\Omega ; \mathbb{R}))$, apresentaremos um resultado similar ao do Teorema do Valor Médio do contexto clássico. Observamos que a partir do estudo do artigo [AFJ-1] nos foi possível acrescentar ao resultado obtido pelos autores, utilizando a Proposição 1.6.4, o caso em que $x, y \in \widetilde{\Omega}_{c}$ e $x \approx y$, onde $\Omega$ é um subconjunto aberto de $\mathbb{R}^{n}$.

Teorema 4.1.6 (Teorema do Valor Médio Generalizado) Sejam $\Omega$ um subconjunto aberto de $\mathbb{R}^{n}, f \in \mathcal{G}(\Omega ; \mathbb{R})$ e $x, y \in \widetilde{\Omega}_{c}$. Se $x \approx y$, ou se $\Omega$ é convexo, então existe $c \in \widetilde{\Omega}_{c}$ tal que

$$
\kappa(f)(x)-\kappa(f)(y)=\langle\nabla \kappa(f)(c), x-y\rangle \quad e \quad|\kappa(f)(x)-\kappa(f)(y)| \leq[\nabla \kappa(f)(c)]_{2}[x-y]_{2} .
$$

Além disso, tem-se que $\max \{\|c-x\|,\|c-y\|\} \leq\|x-y\|$ e existe $K \subset \subset \Omega$ tal que $x, y, c \in \widetilde{K}$.

Demonstração: Sejam $\left(x_{\varepsilon}\right)_{\varepsilon}:=\left(\left(x_{1 \varepsilon}, \ldots, x_{n \varepsilon}\right)\right)_{\varepsilon},\left(y_{\varepsilon}\right)_{\varepsilon}:=\left(\left(y_{1 \varepsilon}, \ldots, y_{n \varepsilon}\right)\right)_{\varepsilon} \in \Omega_{c}$ tais que $x=\left(\left[\left(x_{1 \varepsilon}\right)_{\varepsilon}\right], \ldots,\left[\left(x_{n \varepsilon}\right)_{\varepsilon}\right]\right)$ e $y=\left(\left[\left(y_{1 \varepsilon}\right)_{\varepsilon}\right], \ldots,\left[\left(y_{n \varepsilon}\right)_{\varepsilon}\right]\right)$. Se $x \approx y$, temos, por (1.2), que

$$
\lim _{\varepsilon \downarrow 0}\left|x_{\varepsilon}-y_{\varepsilon}\right|=0
$$

Portanto, se $x \approx y$, ou se $\Omega$ é convexo, temos, pela Proposição 1.6.4, que existem $K \subset \subset \Omega$ e $\eta \in \mathbf{I}$ tais que $P_{\varepsilon} \subset K$, para todo $\varepsilon \in \mathbf{I}_{\eta}$, onde $P_{\varepsilon}$ é o segmento de extremidades $x_{\varepsilon}$ e $y_{\varepsilon}$.

Seja $\left(f_{\varepsilon}\right)_{\varepsilon}$ um representante de $f$. Notemos que $f_{\varepsilon}$ é diferenciável, para todo $\varepsilon \in \mathbf{I}$, pois $\left(f_{\varepsilon}\right)_{\varepsilon} \in\left(\mathscr{C}^{\infty}(\Omega ; \mathbb{R})\right)^{\mathbf{I}}$. Desse modo, para cada $\varepsilon \in \mathbf{I}_{\eta}$ fixado, temos, pelo Teorema do Valor Médio, que existe $z_{\varepsilon} \in P_{\varepsilon} \subset K \subset \Omega$ tal que

$$
f_{\varepsilon}\left(x_{\varepsilon}\right)-f_{\varepsilon}\left(y_{\varepsilon}\right)=\sum_{i=1}^{n} \frac{\partial f}{\partial x_{i}}\left(z_{\varepsilon}\right)\left(x_{i \varepsilon}-y_{i \varepsilon}\right) .
$$


Seja, para cada $\varepsilon \in \mathbf{I}$,

$$
c_{\varepsilon}:=\left\{\begin{array}{ll}
z_{\varepsilon} & , \text { se } 0<\varepsilon<\eta \\
z_{\frac{\eta}{2}} & , \eta \leq \varepsilon \leq 1
\end{array} .\right.
$$

Então $\left(c_{\varepsilon}\right)_{\varepsilon} \in \Omega_{c}$ e, portanto, podemos considerar $c:=\left[\left(c_{\varepsilon}\right)_{\varepsilon}\right] \in \widetilde{K}$. Notemos também que $x, y \in \widetilde{K}$. Dessa forma, por (4.5) e pela Proposição 4.1.4, temos que $\kappa(f)(x)-\kappa(f)(y)=\sum_{i=1}^{n} \kappa\left(\frac{\partial f}{\partial x_{i}}\right)(c)\left(x_{i}-y_{i}\right)=\sum_{i=1}^{n} \frac{\partial(\kappa(f))}{\partial x_{i}}(c)\left(x_{i}-y_{i}\right)=\langle\nabla \kappa(f)(c), x-y\rangle$.

Logo, pela desigualdade de Cauchy-Schwarz generalizada (Lema 1.4.15), temos que

$$
|\kappa(f)(x)-\kappa(f)(y)| \leq[\nabla \kappa(f)(c)]_{2}[x-y]_{2} .
$$

Finalmente, notemos que, como $c_{\varepsilon} \in P_{\varepsilon}$, para todo $\varepsilon \in \mathbf{I}$, temos que

$$
\left|c_{j \varepsilon}-x_{j \varepsilon}\right| \leq\left|x_{j \varepsilon}-y_{j \varepsilon}\right| \quad \text { e } \quad\left|c_{j \varepsilon}-y_{j \varepsilon}\right| \leq\left|x_{j \varepsilon}-y_{j \varepsilon}\right|, \forall \varepsilon \in \mathbf{I}, \forall 1 \leq j \leq n .
$$

Assim,

$$
\left|c_{j}-x_{j}\right| \leq\left|x_{j}-y_{j}\right| \quad \text { e } \quad\left|c_{j}-y_{j}\right| \leq\left|x_{j}-y_{j}\right|, \forall 1 \leq j \leq n .
$$

Portanto, pela Proposição 1.3.7 (10), obtemos que

$$
\begin{aligned}
& \|c-x\|=\max \left\{\left\|c_{j}-x_{j}\right\|: 1 \leq j \leq n\right\} \leq \max \left\{\left\|x_{j}-y_{j}\right\|: 1 \leq j \leq n\right\}=\|x-y\| ; \\
& \|c-y\|=\max \left\{\left\|c_{j}-y_{j}\right\|: 1 \leq j \leq n\right\} \leq \max \left\{\left\|x_{j}-y_{j}\right\|: 1 \leq j \leq n\right\}=\|x-y\| ;
\end{aligned}
$$

ou seja,

$$
\max \{\|c-x\|,\|c-y\|\} \leq\|x-y\| .
$$

O próximo resultado é uma aplicação do Teorema do Valor Médio Generalizado e nos mostra que as funções pertencentes a $\kappa(\mathcal{G}(\Omega ; \mathbb{R}))$ são sempre diferenciáveis em $\widetilde{\Omega}_{c}$.

Proposição 4.1.7 Seja $\Omega$ um subconjunto aberto de $\mathbb{R}^{n}$. Se $f \in \mathcal{G}(\Omega ; \mathbb{R})$, então $\kappa(f)$ é diferenciável em $\tilde{\Omega}_{c}$. 
Demonstração: Consideremos $c=\left(c_{1}, \ldots, c_{n}\right):=\left(\left[\left(c_{1 \varepsilon}\right)_{\varepsilon}\right], \ldots,\left[\left(c_{n \varepsilon}\right)_{\varepsilon}\right]\right) \in \widetilde{\Omega}_{c}$, onde $\left(c_{\varepsilon}\right)_{\varepsilon}:=$ $\left(\left(c_{1 \varepsilon}, \ldots, c_{n \varepsilon}\right)\right)_{\varepsilon} \in \Omega_{c}$. Provaremos que

$$
\lim _{x \rightarrow c}\left\|\frac{\kappa(f)(x)-\kappa(f)(c)-\sum_{i=1}^{n} \kappa\left(\partial_{i} f\right)(c)\left(x_{i}-c_{i}\right)}{\alpha_{-\ln \|x-c\|}}\right\|=0,
$$

onde $x_{i}$ denota a i-ésima coordenada de $x$. Assim, pela Proposição 2.3.5 e pela Proposição 4.1.4, obteremos que $\kappa(f)$ é diferenciável em $c$.

Seja $x=\left(x_{1}, \ldots, x_{n}\right):=\left(\left[\left(x_{1 \varepsilon}\right)_{\varepsilon}\right], \ldots,\left[\left(x_{n \varepsilon}\right)_{\varepsilon}\right]\right) \in \widetilde{\Omega}_{c}$, onde $\left(x_{\varepsilon}\right)_{\varepsilon}:=\left(\left(x_{1 \varepsilon}, \ldots, x_{n \varepsilon}\right)\right)_{\varepsilon} \in \Omega_{c}$, com $\|x-c\|<1$. Então $x-c \approx 0$ (Proposição 1.4.5 (1)(a)). Desse modo, pelo Teorema do Valor Médio Generalizado (Teorema 4.1.6) e pela Proposição 4.1.4, existe $y=\left(y_{1}, \ldots, y_{n}\right):=$ $\left(\left[\left(y_{1 \varepsilon}\right)_{\varepsilon}\right], \ldots,\left[\left(y_{n \varepsilon}\right)_{\varepsilon}\right]\right) \in \widetilde{\Omega}_{c}$, onde $\left(y_{\varepsilon}\right)_{\varepsilon}:=\left(\left(y_{1 \varepsilon}, \ldots, y_{n \varepsilon}\right)\right)_{\varepsilon} \in \Omega_{c}$, tal que

$$
\|y-c\| \leq\|x-c\| \quad \text { e } \quad \kappa(f)(x)-\kappa(f)(c)=\sum_{i=1}^{n} \kappa\left(\partial_{i} f\right)(y)\left(x_{i}-c_{i}\right) .
$$

De (4.6), temos que

$$
\left\|\kappa(f)(x)-\kappa(f)(c)-\sum_{i=1}^{n} \kappa\left(\partial_{i} f\right)(c)\left(x_{i}-c_{i}\right)\right\|=\left\|\sum_{i=1}^{n}\left(\kappa\left(\partial_{i} f\right)(y)-\kappa\left(\partial_{i} f\right)(c)\right)\left(x_{i}-c_{i}\right)\right\| .
$$

Seja $A_{i y}:=\kappa\left(\partial_{i} f\right)(y)-\kappa\left(\partial_{i} f\right)(c)$, para todo $1 \leq i \leq n$. Notemos que, pela Proposição 1.3.7 (1) e (2), temos que

$$
\left\|\sum_{i=1}^{n} A_{i y}\left(x_{i}-c_{i}\right)\right\| \leq \max \left\{\left\|A_{i y}\right\|\left\|x_{i}-c_{i}\right\|: 1 \leq i \leq n\right\} \leq\|x-c\| \max \left\{\left\|A_{i y}\right\|: 1 \leq i \leq n\right\} .
$$

Consideremos $E(x):=\kappa(f)(x)-\kappa(f)(c)-\sum_{i=1}^{n} \kappa\left(\partial_{i} f\right)(c)\left(x_{i}-c_{i}\right)$. Logo, pela Proposição 1.3.7 (5) e por (4.7), obtemos que

$$
\left\|\frac{E(x)}{\alpha_{-\ln \|x-c\|}}\right\|=\frac{\|E(x)\|}{\|x-c\|} \leq \max \left\{\left\|A_{i y}\right\|: 1 \leq i \leq n\right\} .
$$

Observemos que, pelo fato de $\|y-c\| \leq\|x-c\|$ e da continuidade de $\kappa\left(\partial_{i} f\right)$ (Proposição 4.1.3), obtemos que $\lim _{x \rightarrow c} \max \left\{\left\|A_{i y}\right\|: 1 \leq i \leq n\right\}=0$. Portanto, 


$$
\lim _{x \rightarrow c}\left\|\frac{\kappa(f)(x)-\kappa(f)(c)-\sum_{i=1}^{n} \kappa\left(\partial_{i} f\right)(c)\left(x_{i}-c_{i}\right)}{\alpha_{-\ln \|x-c\|}}\right\|=0 .
$$

No capítulo 1, definimos o que significa uma função generalizada $f$ ser constante (Definição 1.5.8) e vimos, no capítulo 2, que uma função pode ser derivável em $\overline{\mathbb{R}}$, ter derivada nula e não ser constante (Exemplo $2.2 .4(2)$ ). Contudo, como nos mostra a próxima Proposição, isso não ocorre para funções pertencentes a $\kappa(\mathcal{G}(\Omega ; \mathbb{R}))$, no caso em que $\Omega$ é um subconjunto aberto conexo de $\mathbb{R}^{n}$.

Proposição 4.1.8 Sejam $\Omega$ um subconjunto aberto de $\mathbb{R}^{n}$ e $f \in \mathcal{G}(\Omega ; \mathbb{R})$. Se $\Omega$ é conexo e $D(\kappa(f))=0$, então $f$ é constante. Em particular, $\kappa(f)$ é uma função constante.

Demonstração: Por hipótese, tem-se que $D(\kappa(f))=0$. Então, pela Observação 2.4.3 e pela Proposição 4.1.4, temos que existem as derivadas parciais de $\kappa(f) \mathrm{e}$

$$
\kappa\left(\frac{\partial f}{\partial x_{i}}\right)=\frac{\partial(\kappa(f))}{\partial x_{i}}=0, \quad \forall 1 \leq i \leq n .
$$

Desse modo, como $\kappa$ é homomorfismo injetor (Proposição 4.1.2), obtemos que $\frac{\partial f}{\partial x_{i}}=0$, para todo $1 \leq i \leq n$, ou seja, $\nabla f=0$. Logo, da conexidade de $\Omega$, concluímos que $f$ é constante (Proposição 1.5.12). Assim, existe $\lambda \in \overline{\mathbb{R}}$ tal que $f=\left[\left(\lambda_{\varepsilon}^{*}\right)_{\varepsilon}\right]$, onde $\left(\lambda_{\varepsilon}\right)_{\varepsilon}$ é um representante de $\lambda$ e $\lambda_{\varepsilon}^{*}(x)=\lambda_{\varepsilon}$, para todo $\varepsilon \in \mathbf{I}$ e todo $x \in \Omega$. Portanto, $\kappa(f)(y)=f(y)=\left[\left(\lambda_{\varepsilon}^{*}\left(y_{\varepsilon}\right)\right)_{\varepsilon}\right]=$ $\left[\left(\lambda_{\varepsilon}\right)_{\varepsilon}\right]=\lambda$, para todo $y=\left(\left[\left(y_{1 \varepsilon}\right)_{\varepsilon}\right], \ldots,\left[\left(y_{n \varepsilon}\right)_{\varepsilon}\right]\right) \in \tilde{\Omega}_{c}$, onde $\left(y_{\varepsilon}\right)_{\varepsilon}:=\left(\left(y_{1 \varepsilon}, \ldots, y_{n \varepsilon}\right)\right)_{\varepsilon} \in \Omega_{c}, \mathrm{o}$ que prova a última afirmação.

A seguir, apresentaremos uma aplicação da Proposição anterior.

Proposição 4.1.9 Sejam $\Omega$ um subconjunto aberto conexo de $\mathbb{R}^{n}$ e $f \in \mathcal{G}(\Omega ; \mathbb{R})$. Se o conjunto $\left\{\kappa(f)(x): x \in \widetilde{\Omega}_{c}\right\}$ é um subconjunto discreto de $\overline{\mathbb{R}}$, então $f$ é constante. 
Demonstração: Observemos que é suficiente mostrarmos, pela Proposição 4.1.8, que $D(\kappa(f))=0$. Para isso, mostraremos que, se $x_{0} \in \widetilde{\Omega}_{c}$, então $\kappa(f)$ é constante em uma vizinhança de $x_{0}$ e, portanto, $D(\kappa(f))\left(x_{0}\right)=0$.

Seja $x_{0} \in \widetilde{\Omega}_{c}$. Por hipótese, o conjunto $\left\{\kappa(f)(x): x \in \widetilde{\Omega}_{c}\right\}$ é discreto. Então existe $s>0$ tal que

$$
B_{s}\left(\kappa(f)\left(x_{0}\right)\right) \cap \kappa(f)\left(\tilde{\Omega}_{c}\right)=\left\{\kappa(f)\left(x_{0}\right)\right\}
$$

Da continuidade de $\kappa(f)$ (Proposição 4.1.3), existe $\delta>0$ tal que, se $y \in \widetilde{\Omega}_{c}$ e $0<\left\|y-x_{0}\right\|<\delta$, então $\left\|\kappa(f)(y)-\kappa(f)\left(x_{0}\right)\right\|<s$. Logo, por (4.8), concluímos que $\kappa(f)(y)=\kappa(f)\left(x_{0}\right)$, para todo $y \in B_{\delta}\left(x_{0}\right) \cap \widetilde{\Omega}_{c}$, ou seja, que $\left.\kappa(f)\right|_{B_{\delta}\left(x_{0}\right) \cap \tilde{\Omega}_{c}}$ é constante.

No contexto clássico, se $f$ é uma função contínua e $L \subset \subset \operatorname{Dom}(f)$, então $\left.f\right|_{L}$ é uma função limitada. Na próxima Proposição, apresentaremos um resultado similar utilizando o conjunto $\widetilde{K}$, onde $K \subset \subset \Omega$.

Proposição 4.1.10 Sejam $\Omega$ um subconjunto aberto de $\mathbb{R}^{n}$ e $f \in \mathcal{G}(\Omega ; \mathbb{R})$. Se $K \subset \subset \Omega$, então tem-se que:

1. a função $\left.\kappa(f)\right|_{\widetilde{K}}$ é limitada, isto é, existe $M_{1}>0$ tal que

$$
\|\kappa(f)(x)\| \leq M_{1}, \forall x \in \widetilde{K}
$$

2. se $0 \in \Omega$, então $\left.\kappa(f)\right|_{\widetilde{K} \cap B_{1}(0)}$ é uma função Lipschitziana, isto é, existe $M_{2}>0$ tal que

$$
\|\kappa(f)(x)-\kappa(f)(y)\| \leq M_{2}\|x-y\|, \forall x, y \in \widetilde{K} \cap B_{1}(0) .
$$

Demonstração: Primeiramente, vamos mostrar que $\left.\kappa(f)\right|_{\widetilde{K}}$ é limitada.

Seja $\left(f_{\varepsilon}\right)_{\varepsilon}$ um representante de $f$. Da moderação de $\left(f_{\varepsilon}\right)_{\varepsilon}$, existem $c>0, N \in \mathbb{N}$ e $\eta \in \mathbf{I}$ tais que

$$
\left|f_{\varepsilon}(y)\right| \leq c \varepsilon^{-N}, \forall \varepsilon \in \mathbf{I}_{\eta}, \forall y \in K
$$


Seja $z \in \widetilde{K}$. Então existe $\left(z_{\varepsilon}\right)_{\varepsilon}:=\left(\left(z_{1 \varepsilon}, \ldots, z_{n \varepsilon}\right)\right) \in \Omega_{c}$ e $\nu \in \mathbf{I}_{\eta}$ tais que $z=$ $\left(\left[\left(z_{1 \varepsilon}\right)_{\varepsilon}\right], \ldots,\left[\left(z_{n \varepsilon}\right)_{\varepsilon}\right]\right)$ e $z_{\varepsilon} \in K$, para todo $\varepsilon \in \mathbf{I}_{\nu}$. Logo, temos que $\left|f_{\varepsilon}\left(z_{\varepsilon}\right)\right| \leq c \varepsilon^{-N}$, para todo $\varepsilon \in \mathbf{I}_{\nu}$, e, assim,

$$
|\kappa(f)(z)| \leq c \alpha_{-N}=\left|c \alpha_{-N}\right|
$$

Portanto, pela Proposição 1.3.7 (4), (8) e (10), segue que

$$
\|\kappa(f)(z)\| \leq\left\|c \alpha_{-N}\right\|=e^{N}
$$

Como $z \in \widetilde{K}$ foi escolhido arbitrariarmente, obtemos 1 ..

A seguir, mostraremos que $\left.\kappa(f)\right|_{\widetilde{K}_{\cap} B_{1}(0)}$ é uma função Lipschitziana.

Consideremos $V$ um subconjunto aberto de $\Omega$ tal que $K \subset V \subset \bar{V} \subset \subset \Omega$ e seja $r:=\frac{1}{2} \operatorname{dist}\left(K, V^{c}\right)>0$. Então

$$
B_{r}(a) \subset V \subset \bar{V} \subset \subset \Omega, \forall a \in K
$$

Seja $L:=\bar{V}$. Para cada $1 \leq i \leq n$, temos, por 1 ., que $\left.\kappa\left(\partial_{i} f\right)\right|_{\widetilde{L}}$ é limitada, ou seja, existe $M_{i}>0$ tal que

$$
\left\|\kappa\left(\partial_{i} f\right)(w)\right\| \leq M_{i}, \forall w \in \widetilde{L}
$$

Consideremos $M:=\max \left\{M_{i}: 1 \leq i \leq n\right\}$.

Sejam $x=\left(x_{1}, \ldots, x_{n}\right):=\left(\left[\left(x_{1 \varepsilon}\right)_{\varepsilon}\right], \ldots,\left[\left(x_{n \varepsilon}\right)_{\varepsilon}\right]\right), y=\left(y_{1}, \ldots, y_{n}\right):=\left(\left[\left(y_{1 \varepsilon}\right)_{\varepsilon}\right], \ldots,\left[\left(y_{n \varepsilon}\right)_{\varepsilon}\right]\right)$ $\in \widetilde{K} \cap B_{1}(0)$, onde $\left(x_{\varepsilon}\right)_{\varepsilon}:=\left(\left(x_{1 \varepsilon}, \ldots, x_{n \varepsilon}\right)\right)_{\varepsilon} \in \Omega_{c}$ e $\left(y_{\varepsilon}\right)_{\varepsilon}:=\left(\left(y_{1 \varepsilon}, \ldots, y_{n \varepsilon}\right)\right)_{\varepsilon} \in \Omega_{c}$ são tais que existe $\nu_{1} \in \mathbf{I}$ de modo que $x_{\varepsilon}, y_{\varepsilon} \in K$, para todo $\varepsilon \in \mathbf{I}_{\nu_{1}}$.

Como $\|x-y\| \leq \max \{\|x\|,\|y\|\}<1$ (Definição 1.3 .10 e Proposição 1.3 .7 (1) e (4)), temos, pela Proposição $1.4 .5(1)(\mathrm{a})$, que $x \approx y$. Portanto, por (1.2), obtemos que

$$
\lim _{\varepsilon \downarrow 0}\left|x_{\varepsilon}-y_{\varepsilon}\right|=0
$$

Logo, existe $\nu_{2} \in \mathbf{I}$ tal que

$$
\left|x_{\varepsilon}-y_{\varepsilon}\right|<r, \forall \varepsilon \in \mathbf{I}_{\nu_{2}} \text {. }
$$

Sejam $\left(f_{\varepsilon}\right)_{\varepsilon}$ um representante de $f$ e $\eta:=\min \left\{\nu_{1}, \nu_{2}\right\}$. Fixemos $\varepsilon \in \mathbf{I}_{\eta}$. Como $x_{\varepsilon}, y_{\varepsilon} \in B_{r}\left(x_{\varepsilon}\right) \subset L \subset \Omega$ e $f_{\varepsilon} \in \mathscr{C}^{\infty}(\Omega ; \mathbb{R})$, existe, pelo Teorema do Valor Médio, 
$c_{\varepsilon} \in B_{r}\left(x_{\varepsilon}\right) \subset L \subset \Omega$ tal que

$$
f\left(x_{\varepsilon}\right)-f\left(y_{\varepsilon}\right)=\sum_{i=1}^{n} \partial_{i} f_{\varepsilon}\left(c_{\varepsilon}\right)\left(x_{i \varepsilon}-y_{i \varepsilon}\right)
$$

e, assim,

$$
\left|f\left(x_{\varepsilon}\right)-f\left(y_{\varepsilon}\right)\right| \leq \sum_{i=1}^{n}\left|\partial_{i} f_{\varepsilon}\left(c_{\varepsilon}\right)\right|\left|x_{i \varepsilon}-y_{i \varepsilon}\right| .
$$

Seja, para cada $\varepsilon \in \mathbf{I}$,

$$
z_{\varepsilon}:=\left\{\begin{array}{ll}
c_{\varepsilon} & , \text { se } 0<\varepsilon<\eta \\
c_{\frac{\eta}{2}} & , \eta \leq \varepsilon \leq 1
\end{array} .\right.
$$

Então $\left(z_{\varepsilon}\right)_{\varepsilon}:=\left(\left(z_{1 \varepsilon}, \ldots, z_{n \varepsilon}\right)\right)_{\varepsilon} \in \Omega_{c}, z:=\left(\left[\left(z_{1 \varepsilon}\right)_{\varepsilon}\right], \ldots,\left[\left(z_{n \varepsilon}\right)_{\varepsilon}\right]\right) \in \tilde{L}$ e, por $(4.9)$, temos que

$$
|\kappa(f)(x)-\kappa(f)(y)| \leq\left|\sum_{i=1}^{n}\right| \kappa\left(\partial_{i} f\right)(z)|| x_{i}-y_{i}|| .
$$

Logo, por (4.10) e pela Proposição $1.3 .7(1),(2),(9)$ e (10), temos que

$$
\begin{aligned}
\|\kappa(f)(x)-\kappa(f)(y)\| & \leq \max \left\{\left\|\kappa\left(\partial_{i} f\right)(z)\right\|\left\|x_{i}-y_{i}\right\|: 1 \leq i \leq n\right\} \\
& \leq M \max \left\{\left\|x_{i}-y_{i}\right\|: 1 \leq i \leq n\right\} \\
& =M\|x-y\| .
\end{aligned}
$$

Em seguida, apresentaremos um resultado similar à Fórmula de Taylor do contexto clássico no caso em que $n=1$.

Proposição 4.1.11 (Fórmula de Taylor Generalizada) Sejam $\Omega$ um subconjunto aberto de $\mathbb{R}, f \in \mathcal{G}(\Omega ; \mathbb{R}), m \in \mathbb{N}^{*}$ e $x, y \in \widetilde{\Omega}_{c}$. As seguintes afirmações são válidas:

1. se $x \approx y$, ou se $\Omega$ é convexo, então existe $c \in \widetilde{\Omega}_{c}$ tal que $\max \{\|c-x\|$, $\|c-y\|\} \leq$ $\|x-y\| e$

$$
\kappa(f)(x)=\sum_{j=0}^{m}\left(\frac{\kappa\left(f^{(j)}\right)(y)(x-y)^{j}}{j !}\right)+\frac{\kappa\left(f^{(m+1)}\right)(c)(x-y)^{m+1}}{(m+1) !}
$$


2.

$$
\lim _{h \rightarrow 0} \frac{1}{\left(\alpha_{-\ln \|h\|}\right)^{m}}\left(\kappa(f)(x+h)-\sum_{j=0}^{m} \frac{\kappa\left(f^{(j)}\right)(x) h^{j}}{j !}\right)=0 .
$$

Demonstração: Sejam $\left(f_{\varepsilon}\right)_{\varepsilon}$ um representante de $f$ e $\left(x_{\varepsilon}\right)_{\varepsilon},\left(y_{\varepsilon}\right)_{\varepsilon} \in \Omega_{c}$ tais que $x=\left[\left(x_{\varepsilon}\right)_{\varepsilon}\right]$ e $y=\left[\left(y_{\varepsilon}\right)_{\varepsilon}\right]$. Notemos que $f_{\varepsilon}$ é diferenciável, para todo $\varepsilon \in \mathbf{I}$, pois $\left(f_{\varepsilon}\right)_{\varepsilon} \in\left(\mathscr{C}^{\infty}(\Omega ; \mathbb{R})\right)^{\mathbf{I}}$.

Provaremos, a seguir, a afirmação 1 ..

Se $x \approx y$ (e, portanto, $\lim _{\varepsilon \downarrow 0}\left|x_{\varepsilon}-y_{\varepsilon}\right|=0$ ), ou se $\Omega$ é convexo, existem $K \subset \subset \Omega$ e $\eta \in \mathbf{I}$ tais que $P_{\varepsilon} \subset K$, para todo $\varepsilon \in \mathbf{I}_{\eta}$, onde $P_{\varepsilon}$ é o segmento de extremidades $x_{\varepsilon}$ e $y_{\varepsilon}$ (Proposição 1.6.4). Desse modo, para cada $\varepsilon \in \mathbf{I}_{\eta}$ fixado, existe, pela Fórmula de Taylor, $z_{\varepsilon} \in P_{\varepsilon} \subset \Omega$ tal que

$$
f_{\varepsilon}\left(x_{\varepsilon}\right)=\sum_{j=0}^{m}\left(\frac{f_{\varepsilon}^{(j)}\left(y_{\varepsilon}\right)\left(x_{\varepsilon}-y_{\varepsilon}\right)^{j}}{j !}\right)+\frac{f_{\varepsilon}^{(m+1)}\left(z_{\varepsilon}\right)\left(x_{\varepsilon}-y_{\varepsilon}\right)^{m+1}}{(m+1) !} .
$$

Seja, para cada $\varepsilon \in \mathbf{I}$,

$$
c_{\varepsilon}:=\left\{\begin{array}{ll}
z_{\varepsilon} & , \text { se } 0<\varepsilon<\eta \\
z_{\frac{\eta}{2}} & , \eta \leq \varepsilon \leq 1
\end{array} .\right.
$$

Então $\left(c_{\varepsilon}\right)_{\varepsilon} \in \Omega_{c}, c:=\left[\left(c_{\varepsilon}\right)_{\varepsilon}\right] \in \widetilde{\Omega}_{c} \mathrm{e}$

$$
\kappa(f)(x)=\sum_{j=0}^{m}\left(\frac{\kappa\left(f^{(j)}\right)(y)(x-y)^{j}}{j !}\right)+\frac{\kappa\left(f^{(m+1)}\right)(c)(x-y)^{m+1}}{(m+1) !} .
$$

Observemos que, como $c_{\varepsilon} \in P_{\varepsilon}$, para todo $\varepsilon \in \mathbf{I}$, temos que

$$
\left|c_{\varepsilon}-x_{\varepsilon}\right| \leq\left|x_{\varepsilon}-y_{\varepsilon}\right| \quad \text { e } \quad\left|c_{\varepsilon}-y_{\varepsilon}\right| \leq\left|x_{\varepsilon}-y_{\varepsilon}\right|, \forall \varepsilon \in \mathbf{I} .
$$

Assim,

$$
|c-x| \leq|x-y| \quad \text { e } \quad|c-y| \leq|x-y| .
$$

Portanto, pela Proposição 1.3.7 (10), obtemos que

$$
\|c-x\| \leq\|x-y\| \quad \text { e } \quad\|c-y\| \leq\|x-y\|,
$$

ou seja,

$$
\max \{\|c-x\|,\|c-y\|\} \leq\|x-y\|
$$


o que finaliza a prova de $1 .$.

Para provarmos 2., consideremos $h \in \bar{R} \operatorname{com} 0<\|h\|<1$. Então, pela Proposição 1.4.5 (1)(a), temos que $h \approx 0$, o que implica que $x \approx x+h$. Logo, por 1., existe $z_{h} \in \widetilde{\Omega}_{c}$ tal que $\left\|z_{h}-x\right\| \leq\|x-(x+h)\|=\|h\| \mathrm{e}$

$$
\kappa(f)(x+h)=\sum_{j=0}^{m}\left(\frac{\kappa\left(f^{(j)}\right)(x) h^{j}}{j !}\right)+\frac{\kappa\left(f^{(m+1)}\right)\left(z_{h}\right) h^{m+1}}{(m+1) !} .
$$

Logo, pela Proposição 1.3 .7 (2), (4) e (5), temos que

$$
\begin{aligned}
\left\|\frac{1}{\left(\alpha_{-\ln \|h\|)^{m}}\right.}\left(\kappa(f)(x+h)-\sum_{j=0}^{m} \frac{\kappa\left(f^{(j)}\right)(x) h^{j}}{j !}\right)\right\| & =\left\|\frac{1}{\left(\alpha_{-\ln \|h\|}\right)^{m}} \frac{\kappa\left(f^{(m+1)}\right)\left(z_{h}\right) h^{m+1}}{(m+1) !}\right\| \\
& \leq \frac{1}{\|h\|^{m}}\left\|\kappa\left(f^{(m+1)}\right)\left(z_{h}\right)\right\|\|h\|^{m+1} \\
& =\left\|\kappa\left(f^{(m+1)}\right)\left(z_{h}\right)\right\|\|h\| .
\end{aligned}
$$

Observemos que $\lim _{h \rightarrow 0} z_{h}=x$. Então, da continuidade de $\kappa\left(f^{(m+1)}\right)$ (Proposição 4.1.3) e da Proposição $2.1 .1(2)$, obtemos que $\lim _{h \rightarrow 0}\left(\left\|\kappa\left(f^{(m+1)}\right)\left(z_{h}\right)\right\|\|h\|\right)=0$. Portanto,

$$
\lim _{h \rightarrow 0} \frac{1}{\left(\alpha_{-\ln \|h\|}\right)^{m}}\left(\kappa(f)(x+h)-\sum_{j=0}^{m} \frac{\kappa\left(f^{(j)}\right)(x) h^{j}}{j !}\right)=0,
$$

o que prova 2 .

Nos próximos resultados, utilizaremos a seguinte definição:

Seja $V$ um subconjunto de $\mathbb{R}^{n}$. Diz-se que $x=\left(x_{1}, \ldots, x_{n}\right) \in \overline{\mathbb{R}}^{n}$ é um elemento de $\tilde{V}_{c}$ se, e somente se, existem $\left(x_{\varepsilon}\right)_{\varepsilon}:=\left(\left(x_{1 \varepsilon}, \ldots, x_{n \varepsilon}\right)\right)_{\varepsilon} \in\left(\mathcal{E}_{M}(\mathbb{R})\right)^{n}$ tal que $x=$ $\left(\left[\left(x_{1 \varepsilon}\right)_{\varepsilon}\right], \ldots,\left[\left(x_{n \varepsilon}\right)_{\varepsilon}\right]\right)$ e existem $K \subset \subset \mathbb{R}^{n}$ e $\eta \in \mathbf{I}$ de modo que $K \subset V$ e $x_{\varepsilon} \in K$, para todo $\varepsilon \in \mathbf{I}_{\eta}$.

É fácil verificar que essa definição coincide, no caso em que $V$ é um subconjunto aberto de $\mathbb{R}^{n}$, com a Definição 1.6.3.

Finalizamos esta seção apresentando, a seguir, algumas propriedades da função $\kappa(f)$, no caso em que $f \in \mathscr{C}^{\infty}(\Omega ; \mathbb{R})$. 
Proposição 4.1.12 Sejam $\Omega$ um subconjunto aberto de $\mathbb{R}^{n}$ e $f \in \mathscr{C}^{\infty}(\Omega ; \mathbb{R}) \subset \mathcal{G}(\Omega ; \mathbb{R})$. As seguintes afirmações são válidas:

1. $\kappa(f)\left(\widetilde{\Omega}_{c}\right) \subset(\widetilde{f(\Omega)})_{c}$;

2. $\|\kappa(f)(x)\| \leq 1$, para todo $x \in \widetilde{\Omega}_{c} ;$ em particular, $\kappa(f)$ é uma função limitada.

Demonstração: Denotemos $U:=\kappa(f)\left(\widetilde{\Omega}_{c}\right)$ e $V:=f(\Omega)$. Sejam $x:=\left(x_{1}, \ldots, x_{n}\right)=$ $\left(\left[\left(x_{1 \varepsilon}\right)_{\varepsilon}\right], \ldots,\left[\left(x_{n \varepsilon}\right)_{\varepsilon}\right]\right) \in \widetilde{\Omega}_{c}$, onde $\left(x_{\varepsilon}\right)_{\varepsilon}:=\left(\left(x_{1 \varepsilon}, \ldots, x_{n \varepsilon}\right)\right)_{\varepsilon} \in \Omega_{c}$. Então existem $W \subset \subset \Omega$ e $\eta \in \mathbf{I}$ tais que $x_{\varepsilon} \in W$, para todo $\varepsilon \in \mathbf{I}_{\eta}$. Assim, da continuidade de $f$, obtemos que $K:=f(W) \subset f(\Omega)=V$ é compacto em $\mathbb{R}$. Logo, $f\left(x_{\varepsilon}\right) \in K \subset V$, para todo $\varepsilon \in \mathbf{I}_{\eta}$, o que implica que $\kappa(f)(x) \in \tilde{V}_{c}$, o que prova 1 ..

Por 1., temos que $U \subset \widetilde{V}_{c} \subset \widetilde{\mathbb{R}}_{c}$, o que implica, pela Proposição 1.6 .7 (1), que $U \subset B_{1}^{\prime}(0)$. Portanto, 2. é verdadeira.

Diz-se que uma função $f$ definida em um subconjunto aberto $U$ de $\mathbb{R}^{n}$ e a valores em $\mathbb{R}$ é aberta se, e somente se, $f(V)$ é um subconjunto aberto de $\mathbb{R}$, para todo $V$ subconjunto aberto de $U$. Surge naturalmente a seguinte pergunta:

Se $f \in \mathscr{C}^{\infty}(\Omega ; \mathbb{R})$ é uma função aberta, será que $\kappa(f)$ leva subconjuntos abertos de $\widetilde{\Omega}_{c}$ em subconjuntos abertos de $\overline{\mathbb{R}}$ ?

O que se sabe, até o momento, em relação a $\left.\kappa\right|_{\mathscr{C} \infty(\Omega ; R)}$ sobre função aberta é o seguinte:

Teorema 4.1.13 (Teorema da Aplicação Aberta Generalizado) Sejam $\Omega$ um subconjunto aberto de $\mathbb{R}^{n}$ e $f \in \mathscr{C}^{\infty}(\Omega ; \mathbb{R})$ uma aplicação aberta. Tem-se que:

1. $\left.\kappa(f)\left(\widetilde{\Omega}_{c}\right)=(\widetilde{f(\Omega})\right)_{c}$;

2. $\kappa(f)\left(\widetilde{W}_{c}\right)$ é um subconjunto aberto de $\overline{\mathbb{R}}$, para todo $W$ subconjunto aberto de $\Omega$.

Demonstração: Denotemos $U:=\kappa(f)\left(\widetilde{\Omega}_{c}\right)$ e $V:=f(\Omega)$. Pela Proposição 4.1.12, temos que $U \subset \tilde{V}_{c}$. Mostraremos que $\tilde{V}_{c} \subset U$. 
Consideremos $\left(\Omega_{m}\right)_{m \in N}$ uma seqüência exaustiva de abertos de $\Omega$. Notemos que

$$
V=f(\Omega)=f\left(\bigcup_{m \in N} \Omega_{m}\right)=\bigcup_{m \in N} f\left(\Omega_{m}\right),
$$

onde $f\left(\Omega_{m}\right)$ é aberto de $\mathbb{R}$, pois $f$ é uma função aberta. Além disso, da continuidade de $f$, obtemos, para todo $m \in \mathbb{N}$, que $f\left(\bar{\Omega}_{m}\right) \subset \subset f\left(\Omega_{m+1}\right)$, o que implica que $\overline{f\left(\Omega_{m}\right)} \subset \subset$ $f\left(\Omega_{m+1}\right)$, pois $\overline{f\left(\Omega_{m}\right)} \subset \overline{f\left(\bar{\Omega}_{m}\right)}=f\left(\bar{\Omega}_{m}\right)$. Logo,

$$
\left(f\left(\Omega_{m}\right)\right)_{m \in N} \text { é uma seqüência exaustiva de abertos de } V \text {. }
$$

Seja $z=\left[\left(z_{\varepsilon}\right)_{\varepsilon}\right] \in \tilde{V}_{c}$, onde $\left(z_{\varepsilon}\right)_{\varepsilon} \in \Omega_{c}$. Então existem $K_{1} \subset \subset V$ e $\eta_{1} \in \mathbf{I}$ tais que $z_{\varepsilon} \in K_{1}$, para todo $\varepsilon \in \mathbf{I}_{\eta_{1}}$.

De (4.11), existe $s \in \mathbb{N}$ tal que $K_{1} \subset f\left(\Omega_{s}\right)$. Desse modo, $L:=\bar{\Omega}_{s} \subset \subset \Omega$ é tal que $K_{1} \subset f(L)$ e, portanto, $z_{\varepsilon} \in f(L)$, para todo $\varepsilon \in \mathbf{I}_{\eta_{1}}$. Logo, para todo $\varepsilon \in \mathbf{I}_{\eta_{1}}$, existe $y_{\varepsilon} \in L$ tal que $f\left(y_{\varepsilon}\right)=z_{\varepsilon}$.

Consideremos, para cada $\varepsilon \in \mathbf{I}$,

$$
w_{\varepsilon}:=\left\{\begin{array}{ll}
y_{\varepsilon} & , \text { se } 0<\varepsilon<\eta_{1} \\
y_{\frac{\eta_{1}}{2}} & , \eta_{1} \leq \varepsilon \leq 1
\end{array} .\right.
$$

Então, se $\left(w_{\varepsilon}\right)_{\varepsilon}:=\left(\left(w_{1 \varepsilon}, \ldots, w_{n \varepsilon}\right)\right)_{\varepsilon}$, temos que $\left(w_{\varepsilon}\right)_{\varepsilon} \in \Omega_{c}, w:=\left(\left[\left(w_{1 \varepsilon}\right)_{\varepsilon}\right], \ldots,\left[\left(w_{n \varepsilon}\right)_{\varepsilon}\right]\right) \in \widetilde{\Omega}_{c}$ e $\kappa(f)(w)=z$. Portanto, $\tilde{V}_{c} \subset U$, o que prova 1 ..

Para provarmos 2., observemos que, se $W$ é um subconjunto aberto de $\Omega$, então $f(W)$ é um subconjunto aberto de $\mathbb{R}$, pois, por hipótese, $f$ é uma aplicação aberta. Dessa forma, segue, pela Proposição 1.6.7 (2), que $(\widetilde{f(W}))_{c}$ é um subconjunto aberto de $\mathbb{R}$. Notemos que $g:=\left.f\right|_{W}$ é uma aplicação aberta, o que implica, por 1., que

$$
(f \widetilde{(W}))_{c}=(g(\widetilde{W}))_{c}=\kappa(g)\left(\widetilde{W}_{c}\right)=\kappa(f)\left(\widetilde{W}_{c}\right) .
$$

\subsection{A função $\kappa_{\Omega, \mathbb{C}^{\prime}}$ e a álgebra $\kappa_{\Omega, \mathbb{C}^{\prime}}\left(\mathcal{G}\left(\Omega ; \mathbb{C}^{\prime}\right)\right)$}


Nesta seção, estudamos a imersão $\kappa_{\Omega, \mathbb{C}}: \mathcal{G}(\Omega ; \mathbb{C}) \longrightarrow \overline{\mathbb{C}}^{\tilde{\Omega}_{c}}$ e algumas propriedades relacionadas a ela e à álgebra $\kappa_{\Omega, \mathbb{C}}(\mathcal{G}(\Omega ; \mathbb{C}))$, onde $\Omega$ denota um subconjunto aberto de $\mathbb{C}$. Além disso, apresentamos o conjunto das funções holomorfas generalizadas, denotado por $\mathscr{H} \mathcal{G}(\Omega)$, e mostramos que para funções pertencentes a $\kappa_{\Omega, \mathbb{C}}(\mathcal{O}(\Omega)) \subset \mathscr{H} \mathcal{G}(\Omega)$ vale um resultado similar ao do Teorema da Aplicação Aberta Generalizado (Teorema 4.2.9), onde $\mathcal{O}(\Omega)$ é o conjunto das funções holomorfas em $\Omega$ no sentido clássico. Destacamos, ainda, que, se $f \in \kappa_{\Omega, \mathbb{C}}(\mathcal{G}(\Omega ; \mathbb{C}))$, então $f$ é holomorfa em $\tilde{\Omega}_{c}$ se, e somente se, $f \in \kappa_{\Omega, \mathbb{C}}(\mathscr{H} \mathcal{G}(\Omega))$ (Proposição $4.2 .8(3)$ ), e que $f$ é holomorfa em $\widetilde{\Omega}_{c}$ se, e somente se, $f$ é analítica em $\widetilde{\Omega}_{c}$ (Corolário 4.2.11).

As notações utilizadas, a seguir, são as mesmas do capítulo 3, a menos de menção em contrário.

Observamos que, sempre que for conveniente, identificaremos $\Omega$ canonicamente com um subconjunto aberto de $\mathbb{R}^{2}$ e, se $z \in \mathbb{C}$, escreveremos $z=(x, y)$, onde $x:=\operatorname{Re}(z)$, $y:=\mathcal{I} \mathrm{m}(z)$ e $\operatorname{Re}(z)$ e $\mathcal{I m}(z)$ denotam a parte real e a parte imaginária de $z$, respectivamente.

No capítulo 3 , vimos que $\overline{\mathbb{C}}$ é isometricamente isomorfo a $\overline{\mathbb{R}}^{2}$. Assim, se $z=\left[\left(z_{\varepsilon}\right)_{\varepsilon}\right] \in$ $\widetilde{\Omega}_{c}$, onde $\Omega$ é um subconjunto aberto de $\mathbb{C}$, podemos escrever $z=(x, y) \in \tilde{\Omega}_{c} \subset \overline{\mathbb{R}}^{2}$, onde $x:=\operatorname{Re}(z), y:=\operatorname{Im}(z)$ e $\operatorname{Re}(z)$ e $\operatorname{Im}(z)$ denotam a parte real generalizada e a parte imaginária generalizada de $z$, respectivamente (Definição 3.1.2).

Notamos que o conjunto $\mathcal{G}(\Omega ; \mathbb{C})$, onde $\Omega$ é um subconjunto aberto de $\mathbb{C}$, é - identificando $\Omega$ canonicamente com um subconjunto aberto de $\mathbb{R}^{2}$ - o conjunto definido na seção 1.5 .

Definição 4.2.1 Sejam $\Omega$ um subconjunto aberto de $\mathbb{C}$ ef $\in \mathcal{G}(\Omega ; \mathbb{C})$. Para cada $z=$ $(x, y) \in \widetilde{\Omega}_{c}$, chama-se de valor pontual generalizado de $f$ em $z$, e denota-se por $f(z)$, o 
elemento de $\overline{\mathbb{C}}$ dado por

$$
f(z):=f(x, y)
$$

onde $f(x, y)$ é o valor pontual generalizado de $f$ em $(x, y)$ definido na Definição 1.6.5.

Em virtude das considerações acima, faz sentido a definição a seguir.

Definição 4.2.2 Seja $\Omega$ um subconjunto aberto de $\mathbb{C}$. Define-se

$$
\begin{aligned}
\kappa_{\Omega, \mathbb{C}}: \mathcal{G}(\Omega ; \mathbb{C}) & \longrightarrow \overline{\mathbb{C}}^{\tilde{\Omega}_{c}} \\
f & \longmapsto \kappa_{\Omega, \mathbb{C}}(f)
\end{aligned},
$$

onde $\kappa_{\Omega, \mathbb{C}}(f)(z):=f(z)$, para todo $z=(x, y) \in \widetilde{\Omega}_{c}$, e $f(z)$ é o valor pontual generalizado de $f$ em $z$ definido na Definição 4.2.1.

Proposição 4.2.3 Seja $\Omega$ um subconjunto aberto de $\mathbb{C}$. A função $\kappa_{\Omega, \mathbb{C}}: \mathcal{G}(\Omega ; \mathbb{C}) \longrightarrow \overline{\mathbb{C}}^{\widetilde{\Omega}_{c}}$, definida na Definição 4.2.2, é um homomorfismo injetor de $\overline{\mathbb{C}}$-álgebras. Além disso, $\kappa_{\Omega, \mathbb{C}}$ é contínua.

Demonstração: Análoga à demonstração da Proposição 4.1.2, substituindo $\mathbb{R}$ e $\overline{\mathbb{R}}$ por $\mathbb{C}$ e $\overline{\mathbb{C}}$, respectivamente, e a Proposição 1.2 .9 (4) pela seguinte afirmação (cuja prova é imediata): se $x \in \overline{\mathbb{C}}$ e $r \in \mathbb{R}$, então $\alpha_{-r}^{*}|x| \approx_{\bar{R}} 0$ se, e somente se, $\alpha_{-r} x \approx_{\overline{\mathbb{C}}} 0$.

Da Proposição 4.2 .3 temos que $\kappa_{\Omega, \mathbb{C}}(\mathcal{G}(\Omega ; \mathbb{C}))$ é uma $\overline{\mathbb{C}}$-álgebra, onde $\Omega$ é um subconjunto aberto de $\mathbb{C}$.

Seja $\Omega$ um subconjunto aberto de $\mathbb{C}$. A seguir, para $f \in \mathcal{G}(\Omega ; \mathbb{C})$, apresentaremos uma outra forma de expressar a função $\kappa_{\Omega, \mathbb{C}}(f)$ utilizando a função $\kappa_{\Omega, \boldsymbol{R}}$ e as funções generalizadas $\mathscr{R} e(f)$ e $\mathscr{I} m(f)$ que serão definidas na Definição 4.2.5. Para tanto, necessitamos dos próximos resultados. 
No que segue, se $\Omega$ é um subconjunto aberto de $\mathbb{C}$ e $f$ é uma função definida em $\Omega$ e a valores em $\mathbb{C}$, então $\mathcal{R e}(f)$ e $\mathcal{I} \mathrm{m}(f)$ denotam a parte real e a parte imaginária de $f$, respectivamente.

Proposição 4.2.4 Sejam $\Omega$ um subconjunto aberto de $\mathbb{C} e\left(f_{\varepsilon}\right)_{\varepsilon},\left(g_{\varepsilon}\right)_{\varepsilon} \in \mathcal{E}[\Omega ; \mathbb{C}]$. Tem-se que:

1. $\left(f_{\varepsilon}\right)_{\varepsilon} \in \mathcal{E}_{M}[\Omega ; \mathbb{C}]$ se, e somente se, $\left(\mathcal{R e}\left(f_{\varepsilon}\right)\right)_{\varepsilon} \in \mathcal{E}_{M}[\Omega ; \mathbb{R}]$ e $\left(\mathcal{I} \mathrm{m}\left(f_{\varepsilon}\right)\right)_{\varepsilon} \in \mathcal{E}_{M}[\Omega ; \mathbb{R}]$;

2. $\left(f_{\varepsilon}\right)_{\varepsilon} \in \mathcal{N}[\Omega ; \mathbb{C}]$ se, e somente se, $\left(\mathcal{R e}\left(f_{\varepsilon}\right)\right)_{\varepsilon} \in \mathcal{N}[\Omega ; \mathbb{R}]$ e $\left(\mathcal{I} \mathrm{m}\left(f_{\varepsilon}\right)\right)_{\varepsilon} \in \mathcal{N}[\Omega ; \mathbb{R}]$;

3. $\left(f_{\varepsilon}-g_{\varepsilon}\right)_{\varepsilon} \in \mathcal{N}[\Omega ; \mathbb{C}]$ se, e somente se, $\left(\mathcal{R e}\left(f_{\varepsilon}\right)-\mathcal{R e}\left(g_{\varepsilon}\right)\right)_{\varepsilon} \in \mathcal{N}[\Omega ; \mathbb{R}]$ e $\left(\mathcal{I} \mathrm{m}\left(f_{\varepsilon}\right)-\mathcal{I} \mathrm{m}\left(g_{\varepsilon}\right)\right)_{\varepsilon} \in \mathcal{N}[\Omega ; \mathbb{R}]$.

Demonstração: Para obtermos 1. e 2., basta notarmos que $\max \left\{\left|\mathcal{R e}\left(f_{\varepsilon}\right)(x)\right|,\left|\mathcal{I} \mathrm{m}\left(f_{\varepsilon}\right)(x)\right|\right\} \leq\left|f_{\varepsilon}(x)\right| \leq\left|\mathcal{R e}\left(f_{\varepsilon}\right)(x)\right|+\left|\mathcal{I} \mathrm{m}\left(f_{\varepsilon}\right)(x)\right|, \forall \varepsilon \in \mathbf{I}, \forall x \in \Omega$ e usarmos a definição de função moderada e de função nula.

Para 3., é suficiente usarmos 2. e observarmos que $\operatorname{Re}\left(f_{\varepsilon}-g_{\varepsilon}\right)=\operatorname{Re}\left(f_{\varepsilon}\right)-\mathcal{R e}\left(g_{\varepsilon}\right)$ e $\mathcal{I m}\left(f_{\varepsilon}-g_{\varepsilon}\right)=\mathcal{I} \mathrm{m}\left(f_{\varepsilon}\right)-\mathcal{I} \mathrm{m}\left(g_{\varepsilon}\right)$, para todo $\varepsilon \in \mathbf{I}$.

Em virtude da Proposição anterior, faz sentido a definição dada a seguir.

Definição 4.2.5 Sejam $\Omega$ um subconjunto aberto de $\mathbb{C}$. Para cada $f \in \mathcal{G}(\Omega ; \mathbb{C})$, chamase de parte real generalizada de $f$ e de parte imaginária generalizada de $f$, e denota-se por $\mathscr{R} e(f)$ e $\mathscr{I} m(f)$, respectivamente, os elementos de $\mathcal{G}(\Omega ; \mathbb{R})$ dados por

$$
\mathscr{R} e(f):=\left[\left(\mathcal{R e}\left(f_{\varepsilon}\right)\right)_{\varepsilon}\right] \quad e \quad \operatorname{I} m(f):=\left[\left(\mathcal{I} \mathrm{m}\left(f_{\varepsilon}\right)\right)_{\varepsilon}\right]
$$

onde $\left(f_{\varepsilon}\right)_{\varepsilon}$ é um representante qualquer de $f$. 
Proposição 4.2.6 Sejam $\Omega$ um subconjunto aberto de $\mathbb{C}$ e $f=\left[\left(f_{\varepsilon}\right)_{\varepsilon}\right] \in \mathcal{G}(\Omega ; \mathbb{C})$. Então $f=0$ se, e somente se, $\mathscr{R} e(f)=0$ e $\mathscr{I}_{m}(f)=0$.

Demonstração: Temos que

$$
\begin{aligned}
f=0 \Longleftrightarrow\left(f_{\varepsilon}\right)_{\varepsilon} \in \mathcal{N}[\Omega ; \mathbb{C}] & \Longleftrightarrow\left(\mathcal{R e}\left(f_{\varepsilon}\right)\right)_{\varepsilon} \in \mathcal{N}[\Omega ; \mathbb{R}] \quad \text { e } \quad\left(\mathcal{I} \mathrm{m}\left(f_{\varepsilon}\right)\right)_{\varepsilon} \in \mathcal{N}[\Omega ; \mathbb{R}] \\
& \Longleftrightarrow \mathscr{R} e(f)=0 \quad \text { e } \quad \mathscr{I} m(f)=0,
\end{aligned}
$$

onde a segunda equivalência decorre da Proposição 4.2 .4 (2).

Notamos que, de acordo com o que foi visto no início da seção 3.2 , se $\Omega$ é um subconjunto aberto de $\mathbb{C}$, então para toda $f \in \mathcal{G}(\Omega ; \mathbb{C})$ e todo $z=(x, y) \in \widetilde{\Omega}_{c}$, podemos escrever

$$
\kappa_{\Omega, \mathbb{C}}(f)(z)=f(x, y)=\operatorname{Re}(f(x, y))+i \operatorname{Im}(f(x, y)) .
$$

Observamos, ainda, que, se $f=\left[\left(f_{\varepsilon}\right)_{\varepsilon}\right]$ e $(x, y)=\left(\left[\left(x_{\varepsilon}\right)_{\varepsilon}\right],\left[\left(y_{\varepsilon}\right)_{\varepsilon}\right]\right)$, então

$$
\begin{gathered}
\operatorname{Re}(f(x, y))=\left(\mathcal{R e}\left(f_{\varepsilon}\left(x_{\varepsilon}, y_{\varepsilon}\right)\right)\right)_{\varepsilon}+\mathcal{N}[\Omega ; \mathbb{R}] ; \\
\operatorname{Im}(f(x, y))=\left(\mathcal{I} \mathrm{m}\left(f_{\varepsilon}\left(x_{\varepsilon}, y_{\varepsilon}\right)\right)\right)_{\varepsilon}+\mathcal{N}[\Omega ; \mathbb{R}] ; \\
\kappa_{\Omega, \boldsymbol{R}}(\mathscr{R} e(f))(x, y)=\mathscr{R} e(f)(x, y)=\left(\mathcal{R e}\left(f_{\varepsilon}\left(x_{\varepsilon}, y_{\varepsilon}\right)\right)\right)_{\varepsilon}+\mathcal{N}[\Omega ; \mathbb{R}] ; \\
\kappa_{\Omega, \boldsymbol{R}}\left(\mathscr{I}_{m}(f)\right)(x, y)=\mathscr{I} m(f)(x, y)=\left(\mathcal{I} \mathrm{m}\left(f_{\varepsilon}\left(x_{\varepsilon}, y_{\varepsilon}\right)\right)\right)_{\varepsilon}+\mathcal{N}[\Omega ; \mathbb{R}] .
\end{gathered}
$$

Logo, temos que

$$
\operatorname{Re}(f(x, y))=\kappa_{\Omega, \boldsymbol{R}}(\mathscr{R} e(f))(x, y) \quad \text { e } \quad \operatorname{Im}(f(x, y))=\kappa_{\Omega, \boldsymbol{R}}\left(\mathscr{I}_{m}(f)\right)(x, y)
$$

De (4.12) e (4.13), obtemos que

$$
\kappa_{\Omega, \mathbb{C}}(f)=\kappa_{\Omega, \boldsymbol{R}}(\mathscr{R} e(f))+i \kappa_{\Omega, \boldsymbol{R}}\left(\mathscr{I}_{m}(f)\right), \quad \forall f \in \mathcal{G}(\Omega ; \mathbb{C})
$$


Nos próximos resultados, utilizaremos a seguinte notação:

Seja $\Omega$ um subconjunto aberto de $\mathbb{C}$. Denota-se por $\mathcal{O}(\Omega)$ o conjunto das funções, definidas em $\Omega$ e a valores em $\mathbb{C}$, que são holomorfas em $\Omega$ no sentido clássico, isto é,

$$
\mathcal{O}(\Omega):=\left\{f \in \mathscr{C}^{1}(\Omega ; \mathbb{C}): \frac{\partial f}{\partial \bar{z}}=0\right\}
$$

onde $\frac{\partial f}{\partial \bar{z}}:=\frac{1}{2}\left\{\frac{\partial f}{\partial x}+i \frac{\partial f}{\partial y}\right\}$ e $i^{2}=-1$

Definição 4.2.7 Seja $\Omega$ um subconjunto aberto de $\mathbb{C}$. Define-se

$$
\mathscr{H} \mathcal{G}(\Omega):=\left\{f \in \mathcal{G}(\Omega ; \mathbb{C}): \frac{\partial f}{\partial \bar{z}}=0 \text { em } \mathcal{G}(\Omega ; \mathbb{U})\right\},
$$

onde $\frac{\partial f}{\partial \bar{z}}:=\frac{1}{2}\left\{\frac{\partial f}{\partial x}+i \frac{\partial f}{\partial y}\right\}, \frac{\partial f}{\partial x}$ e $\frac{\partial f}{\partial y}$ são definidos na Definição 1.5 .9 e $i^{2}=-1$.

Um elemento de $\mathscr{H} \mathcal{G}(\Omega)$ é chamado de função holomorfa generalizada.

Notemos que $\mathcal{O}(\Omega) \subset \mathcal{G}(\Omega ; \mathbb{C})$ e $\mathcal{O}(\Omega) \subset \mathscr{H} \mathcal{G}(\Omega)$. De fato, se $f \in \mathcal{O}(\Omega)$ temos que $f$ é analítica, no sentido clássico, em $\Omega$ e portanto $f \in \mathscr{C}^{\infty}(\Omega ; \mathbb{C}) \subset \mathcal{G}(\Omega ; \mathbb{C})$. A segunda afirmação segue diretamente da Definição 4.2.7. Veremos, em seguida, que $\kappa_{\Omega, \mathbb{C}}(\mathscr{H} \mathcal{G}(\Omega)) \subset \mathscr{H}\left(\tilde{\Omega}_{c}\right)$ (Proposição $\left.4.2 .8(3)\right)$.

Proposição 4.2.8 Sejam $\Omega$ um subconjunto aberto de $\mathbb{C}$ e $f=\left[\left(f_{\varepsilon}\right)_{\varepsilon}\right] \in \mathcal{G}(\Omega ; \mathbb{C})$. Tem-se que:

1. $\frac{\partial f}{\partial \bar{z}}=0 \Longleftrightarrow \frac{\partial(\mathscr{R} e(f))}{\partial x}=\frac{\partial\left(\mathscr{I}_{m}(f)\right)}{\partial y} \quad$ e $\frac{\partial\left(\mathscr{I}_{m}(f)\right)}{\partial x}=-\frac{\partial(\mathscr{R} e(f))}{\partial y} ;$

2. $f \in \mathscr{H} \mathcal{G}(\Omega)$ se, e somente se, $\frac{\partial\left(\kappa_{\Omega, \mathbb{C}}(f)\right)}{\partial \bar{z}}(z)=0$, para todo $z \in \widetilde{\Omega}_{c}$; 
3. $\kappa_{\Omega, \mathbb{C}}(f)$ é holomorfa em $\widetilde{\Omega}_{c}$ se, e somente se, $f \in \mathscr{H} \mathcal{G}(\Omega)$.

Demonstração: A fim de simplificarmos a escrita, denotaremos $\kappa_{\Omega, \mathbb{C}}$ e $\kappa_{\Omega, \boldsymbol{R}}$ por $\kappa_{\mathbb{C}}$ e $\kappa_{\boldsymbol{R}}$, respectivamente.

Para a afirmação 1. é suficiente notarmos que

$$
\begin{aligned}
\frac{\partial f}{\partial \bar{z}} & :=\frac{1}{2}\left\{\frac{\partial f}{\partial x}+i \frac{\partial f}{\partial y}\right\} \\
& =\left(\frac{1}{2}\left\{\left(\frac{\partial\left(\mathcal{R e}\left(f_{\varepsilon}\right)\right)}{\partial x}+i \frac{\partial\left(\mathcal{I} \mathrm{m}\left(f_{\varepsilon}\right)\right)}{\partial x}\right)+i\left(\frac{\partial\left(\mathcal{R e}\left(f_{\varepsilon}\right)\right)}{\partial y}+i \frac{\partial\left(\mathcal{I} \mathrm{m}\left(f_{\varepsilon}\right)\right)}{\partial y}\right)\right\}\right)_{\varepsilon}+\mathcal{N}[\Omega ; \mathbb{C}] \\
& =\left(\frac{1}{2}\left\{\left(\frac{\partial\left(\mathcal{R e}\left(f_{\varepsilon}\right)\right)}{\partial x}-\frac{\partial\left(\mathcal{I} \mathrm{m}\left(f_{\varepsilon}\right)\right)}{\partial y}\right)+i\left(\frac{\partial\left(\mathcal{I} \mathrm{m}\left(f_{\varepsilon}\right)\right)}{\partial x}+\frac{\partial\left(\mathcal{R e}\left(f_{\varepsilon}\right)\right)}{\partial y}\right)\right\}\right)_{\varepsilon}+\mathcal{N}[\Omega ; \mathbb{C}]
\end{aligned}
$$

Assim, pela Proposição 4.2.6, temos que

$$
\begin{aligned}
\frac{\partial f}{\partial \bar{z}}=0 & \Longleftrightarrow \mathscr{R} e\left(\frac{\partial f}{\partial \bar{z}}\right)=0 \quad \text { e } \quad \mathscr{I}_{m}\left(\frac{\partial f}{\partial \bar{z}}\right)=0 \\
& \Longleftrightarrow\left(\frac{\partial\left(\mathcal{R e}\left(f_{\varepsilon}\right)\right)}{\partial x}-\frac{\partial\left(\mathcal{I} \mathrm{m}\left(f_{\varepsilon}\right)\right)}{\partial y}\right)_{\varepsilon}, \quad\left(\frac{\partial\left(\mathcal{I} \mathrm{m}\left(f_{\varepsilon}\right)\right)}{\partial x}+\frac{\partial\left(\mathcal{R e}\left(f_{\varepsilon}\right)\right)}{\partial y}\right)_{\varepsilon} \in \mathcal{N}[\Omega ; \mathbb{R}] \\
& \Longleftrightarrow \frac{\partial(\mathscr{R} e(f))}{\partial x}=\frac{\partial\left(\mathscr{I}_{m}(f)\right)}{\partial y} \text { e } \frac{\partial\left(\mathscr{I}_{m}(f)\right)}{\partial x}=-\frac{\partial(\mathscr{R} e(f))}{\partial y} .
\end{aligned}
$$

Para provarmos 2. e 3., notemos que $\kappa_{\mathbb{C}}(f)=\kappa_{\boldsymbol{R}}(\mathscr{R} e(f))+i \kappa_{\boldsymbol{R}}\left(\mathscr{I}_{m}(f)\right)((4.14))$ e que $\kappa_{\boldsymbol{R}}(\mathscr{R} e(f))$ e $\kappa_{\boldsymbol{R}}\left(\mathscr{I}_{m}(f)\right)$ são diferenciáveis em todo $(x, y) \in \widetilde{\Omega}_{c}$ (Proposição 4.1.7). Portanto, pela Definição 3.2.6, temos que

$$
\begin{aligned}
\frac{\partial\left(\kappa_{\mathbb{C}}(f)\right)}{\partial \bar{z}} & =\frac{1}{2}\left(\frac{\partial\left(\kappa_{\mathbb{C}}(f)\right)}{\partial x}+i \frac{\partial\left(\kappa_{\mathbb{C}}(f)\right)}{\partial y}\right) \\
& =\frac{1}{2}\left(\frac{\partial\left(\kappa_{\boldsymbol{R}}(\mathscr{R} e(f))\right)}{\partial x}+i \frac{\partial\left(\kappa_{\boldsymbol{R}}\left(\mathscr{I}_{m}(f)\right)\right)}{\partial x}\right)+
\end{aligned}
$$




$$
\begin{aligned}
& +\frac{1}{2} i\left(\frac{\partial\left(\kappa_{R}(\mathscr{R} e(f))\right)}{\partial y}+i \frac{\partial\left(\kappa_{R}\left(\mathscr{I}_{m}(f)\right)\right)}{\partial y}\right) \\
= & \frac{1}{2}\left\{\kappa_{\boldsymbol{R}}\left(\frac{\partial(\mathscr{R} e(f))}{\partial x}-\frac{\partial\left(\mathscr{I}_{m}(f)\right)}{\partial y}\right)+i \kappa_{\boldsymbol{R}}\left(\frac{\partial\left(\mathscr{I}_{m}(f)\right)}{\partial x}+\frac{\partial(\mathscr{R} e(f))}{\partial y}\right)\right\},
\end{aligned}
$$

onde a última igualdade decorre do fato de $\kappa_{R}$ ser um homomorfismo (Proposição 4.1.2) e da Proposição 4.1.4. Assim, de 1., do fato de $\kappa_{\boldsymbol{R}}$ ser um homomorfismo injetor (Proposição 4.1.2) e de (4.15), temos que

$$
\begin{aligned}
\frac{\partial f}{\partial \bar{z}}=0 & \Longleftrightarrow \frac{\partial(\mathscr{R} e(f))}{\partial x}-\frac{\partial\left(\mathscr{I}_{m}(f)\right)}{\partial y}=0 \text { e } \frac{\partial\left(\mathscr{I}_{m}(f)\right)}{\partial x}+\frac{\partial(\mathscr{R} e(f))}{\partial y}=0 \\
& \Longleftrightarrow \kappa_{R}\left(\frac{\partial(\mathscr{R} e(f))}{\partial x}-\frac{\partial\left(\mathscr{I}_{m}(f)\right)}{\partial y}\right)=0 \quad \text { e } \quad \kappa_{\boldsymbol{R}}\left(\frac{\partial\left(\mathscr{I}_{m}(f)\right)}{\partial x}+\frac{\partial(\mathscr{R} e(f))}{\partial y}\right)=0 \\
& \Longleftrightarrow \frac{\partial\left(\kappa_{\mathbb{C}}(f)\right)}{\partial \bar{z}}=0 .
\end{aligned}
$$

Portanto, da definição de $\mathscr{H} \mathcal{G}(\Omega)$ e da Proposição 3.2.7, obtemos as afirmações 2. e 3..

Sabe-se que, se $f \in \mathcal{O}(\Omega)$ e $f$ é não-constante, então, para todo subconjunto aberto $W$ de $\Omega$, tem-se que $f(W)$ é um subconjunto aberto de $\mathbb{C}$ (Teorema da Aplicação Aberta). É razoável perguntarmos se existe um resultado análogo para funções em $\mathscr{H}\left(\widetilde{\Omega}_{c}\right)$ ou, pelo menos, para funções em $\kappa_{\Omega, \mathbb{C}}(\mathcal{O}(\Omega)) \subset \mathscr{H}\left(\widetilde{\Omega}_{c}\right)$. O resultado que se tem, nessa direção, é o seguinte:

Teorema 4.2.9 Seja $\Omega$ um subconjunto aberto de $\mathbb{C}$. Se $f \in \mathcal{O}(\Omega)$ é não-constante, então $\kappa_{\Omega, \mathbb{C}}(f)\left(\widetilde{W}_{c}\right)$ é um subconjunto aberto de $\overline{\mathbb{C}}$, para todo subconjunto aberto $W$ de $\Omega$.

Demonstração: Análoga a do Teorema 4.1.13 substituindo $\mathbb{R}$ e $\overline{\mathbb{R}}$ por $\mathbb{C}$ e $\overline{\mathbb{C}}$, respectivamente. 
No contexto clássico, sabe-se que toda função holomorfa é analítica. Não sabemos, até o momento, se essa afirmação é válida no contexto aqui apresentado. Contudo, a partir do próximo Teorema e dos resultados anteriores, provaremos que, se $\Omega$ é um subconjunto aberto de $\mathbb{C}$, então em $\kappa_{\Omega, \mathbb{C}}(\mathscr{H} \mathcal{G}(\Omega))$ os conceitos de função holomorfa e de função analítica são equivalentes (Corolário 4.2.11).

Teorema 4.2.10 Sejam $\Omega$ um subconjunto aberto de $\mathbb{C}$ e $f \in \mathscr{H} \mathcal{G}(\Omega)$. Então $\kappa_{\Omega, \mathbb{C}}(f)$ é analítica em $\widetilde{\Omega}_{c}$.

Demonstração: Seja $\left(f_{\varepsilon}\right)_{\varepsilon}$ um representante de $f$ tal que $f_{\varepsilon}$ é holomorfa, para todo $\varepsilon \in \mathbf{I}$ (esse representante existe por [OPV, Teorema 3]).

Seja $z_{0}=\left[\left(z_{0 \varepsilon}^{1}\right)_{\varepsilon}\right] \in \widetilde{\Omega}_{c} \subset \overline{\mathbb{C}}$, onde $\left(z_{0 \varepsilon}^{1}\right)_{\varepsilon} \in \Omega_{c}$. Então existem $K \subset \subset \Omega$ e $\nu \in \mathbf{I}$ tais que $z_{0 \varepsilon}^{1} \in K$, para todo $\varepsilon \in \mathbf{I}_{\nu}$.

Consideremos, para cada $\varepsilon \in \mathbf{I}$,

$$
z_{0 \varepsilon}:=\left\{\begin{array}{c}
z_{0 \varepsilon}^{1} \quad, \text { se } 0<\varepsilon<\nu \\
z_{0 \frac{\nu}{2}}^{1}, \text { se } \nu \leq \varepsilon \leq 1
\end{array} .\right.
$$

Então $z_{0}=\left[\left(z_{0 \varepsilon}\right)_{\varepsilon}\right]$ e temos que $z_{0 \varepsilon} \in K$, para todo $\varepsilon \in \mathbf{I}$.

Seja $V$ um subconjunto aberto de $\Omega$ tal que $K \subset V \subset \bar{V} \subset \subset \Omega$ e seja $s:=\min \left\{\operatorname{dist}\left(K, V^{c}\right), e^{-2}\right\}>0$. Logo, $\overline{B_{\frac{s}{2}}\left(z_{0 \varepsilon}\right)} \subset \bar{V}$, para todo $\varepsilon \in \mathbf{I}$, e, como $\frac{s}{2}<1$, temos que $B_{\frac{s}{2}}\left(z_{0}\right) \subset \widetilde{\Omega}_{c}$ (Proposição 1.6.7 (1)).

Provaremos que existe $\left(a_{n}\right)_{n \in N}$ seqüência de elementos de $\overline{\mathbb{C}}$ tal que

$$
\kappa_{\Omega, \mathbb{C}}(f)(z)=\sum_{n=0}^{\infty} a_{n}\left(z-z_{0}\right)^{n}, \quad \forall z \in B_{\frac{s}{2}}\left(z_{0}\right)
$$

e, assim, $\kappa_{\Omega, \mathbb{C}}(f)$ será analítica em $z_{0}$.

Sejam $r, \rho \in \mathbb{R}$ tais que $0<\rho<r<\frac{s}{2}$. Para cada $\varepsilon \in \mathbf{I}$, sejam

$$
\begin{aligned}
\gamma_{\varepsilon}(t) & :=z_{0 \varepsilon}+r e^{i t}, \text { onde } 0 \leq t \leq 2 \pi ; \\
a_{n \varepsilon} & :=\frac{1}{2 \pi i} \int_{\gamma_{\varepsilon}} \frac{f_{\varepsilon}(w)}{\left(w-z_{0 \varepsilon}\right)^{n+1}} d w, \text { onde } n \in \mathbb{N} .
\end{aligned}
$$


De $\bar{V} \subset \subset \Omega$ e da moderação de $\left(f_{\varepsilon}\right)_{\varepsilon}$, existem $c>0, N \in \mathbb{N}$ e $\eta_{0} \in \mathbf{I}$ tais que $\left|f_{\varepsilon}(y)\right| \leq c \varepsilon^{-N}$, para todo $\varepsilon \in \mathbf{I}_{\eta_{0}}$ e para todo $y \in \bar{V}$. Então temos que

$$
\left|a_{n \varepsilon}\right| \leq \frac{1}{2 \pi} \int_{\gamma_{\varepsilon}} \frac{c \varepsilon^{-N}}{r^{n+1}}|d w|=\frac{1}{2 \pi} \frac{c \varepsilon^{-N}}{r^{n+1}} 2 \pi r=\frac{c}{r^{n}} \varepsilon^{-N}, \forall \varepsilon \in \mathbf{I}_{\eta_{0}}, \forall n \in \mathbb{N} .
$$

Portanto, $\left(a_{n \varepsilon}\right)_{\varepsilon} \in \mathcal{E}_{M}(\mathbb{C})$, para todo $n \in \mathbb{N}$, o que implica que podemos considerar

$$
a_{n}:=\left[\left(a_{n \varepsilon}\right)_{\varepsilon}\right] \in \overline{\mathbb{C}}, \forall n \in \mathbb{N}
$$

Fixemos $z \in B_{\frac{s}{2}}\left(z_{0}\right)$ e seja $\left(z_{1 \varepsilon}\right)_{\varepsilon}$ um representante de $z$. Como $\left\|z-z_{0}\right\|<\frac{s}{2}<1$, temos, pelo Lema 1.4.5 (1)(a), que $z \approx z_{0}$. Logo, existe $\eta \in \mathbf{I}$ tal que $\left|z_{1 \varepsilon}-z_{0 \varepsilon}\right|<\rho$, para todo $\varepsilon \in \mathbf{I}_{\eta}$.

Seja, para cada $\varepsilon \in \mathbf{I}$,

$$
z_{\varepsilon}:=\left\{\begin{array}{cc}
z_{1 \varepsilon}, \text { se } 0<\varepsilon<\eta \\
z_{0 \varepsilon}, \text { se } \eta \leq \varepsilon \leq 1
\end{array}\right.
$$

Então $\left(z_{\varepsilon}\right)_{\varepsilon}$ é um representante de $z$ tal que

$$
\left|z_{\varepsilon}-z_{0 \varepsilon}\right|<\rho, \forall \varepsilon \in \mathbf{I}
$$

Pela Fórmula Integral de Cauchy, obtemos que

$$
f_{\varepsilon}\left(z_{\varepsilon}\right)=\frac{1}{2 \pi i} \int_{\gamma_{\varepsilon}} \frac{f_{\varepsilon}(w)}{w-z_{\varepsilon}} d w, \quad \forall \varepsilon \in \mathbf{I} .
$$

Observemos que, para todo $\varepsilon \in \mathbf{I}$ e todo $w \in S_{r}\left(z_{0 \varepsilon}\right)$,

$$
\frac{1}{w-z_{\varepsilon}}=\frac{1}{\left(w-z_{0 \varepsilon}\right)-\left(z_{\varepsilon}-z_{0 \varepsilon}\right)}=\frac{1}{\left(w-z_{0 \varepsilon}\right)} \frac{1}{1-\frac{z_{\varepsilon}-z_{0 \varepsilon}}{w-z_{0 \varepsilon}}}=\frac{1}{\left(w-z_{0 \varepsilon}\right)} \sum_{n=0}^{\infty}\left(\frac{z_{\varepsilon}-z_{0 \varepsilon}}{w-z_{0 \varepsilon}}\right)^{n}
$$

onde a última igualdade é válida pois $\left|\frac{z_{\varepsilon}-z_{0 \varepsilon}}{w-z_{0 \varepsilon}}\right|<\frac{\rho}{r}<1$. Logo, temos que

$$
\frac{f_{\varepsilon}(w)}{w-z_{\varepsilon}}=\sum_{n=0}^{\infty} \frac{f_{\varepsilon}(w)}{\left(w-z_{0 \varepsilon}\right)^{n+1}}\left(z_{\varepsilon}-z_{0 \varepsilon}\right)^{n}, \quad \forall w \in S_{r}\left(z_{0 \varepsilon}\right), \forall \varepsilon \in \mathbf{I} .
$$

Notemos, ainda, que $S_{r}\left(z_{0 \varepsilon}\right) \subset \bar{V} \subset \subset \Omega$, para todo $\varepsilon \in \mathbf{I}$. Logo, para cada $\varepsilon \in \mathbf{I}$, existe $M_{\varepsilon}>0$ tal que $\left|f_{\varepsilon}(w)\right| \leq M_{\varepsilon}$, para todo $w \in S_{r}\left(z_{0 \varepsilon}\right)$. Dessa forma, obtemos que

$$
\left|\frac{f_{\varepsilon}(w)}{\left(w-z_{0 \varepsilon}\right)^{n+1}}\left(z_{\varepsilon}-z_{0 \varepsilon}\right)^{n}\right| \leq M_{\varepsilon} \frac{\rho^{n}}{r^{n+1}}, \quad \forall w \in S_{r}\left(z_{0 \varepsilon}\right), \forall \varepsilon \in \mathbf{I},
$$


e, como $\frac{\rho}{r}<1$, temos que $\sum_{n=0}^{\infty} M_{\varepsilon} \frac{\rho^{n}}{r^{n+1}}$ converge. Assim, pelo M-Teste de Weierstrass, concluímos que $\sum_{n=0}^{\infty} \frac{f_{\varepsilon}(w)}{\left(w-z_{0 \varepsilon}\right)^{n+1}}\left(z_{\varepsilon}-z_{0 \varepsilon}\right)^{n}$ converge uniformemente em $S_{r}\left(z_{0 \varepsilon}\right)$, para todo $\varepsilon \in \mathbf{I}$. Portanto, por [Con, Lema 2.7, pg 71], temos, para todo $\varepsilon \in \mathbf{I}$, que

$$
\begin{aligned}
f_{\varepsilon}\left(z_{\varepsilon}\right)=\frac{1}{2 \pi i} \int_{\gamma_{\varepsilon}} \frac{f_{\varepsilon}(w)}{w-z_{\varepsilon}} d w & =\frac{1}{2 \pi i} \int_{\gamma_{\varepsilon}}\left(\sum_{n=0}^{\infty} \frac{f_{\varepsilon}(w)}{\left(w-z_{0 \varepsilon}\right)^{n+1}}\left(z_{\varepsilon}-z_{0 \varepsilon}\right)^{n}\right) d w \\
& =\sum_{n=0}^{\infty}\left(\frac{1}{2 \pi i} \int_{\gamma_{\varepsilon}} \frac{f_{\varepsilon}(w)}{\left(w-z_{0 \varepsilon}\right)^{n+1}} d w\right)\left(z_{\varepsilon}-z_{0 \varepsilon}\right)^{n} \\
& =\sum_{n=0}^{\infty} a_{n \varepsilon}\left(z_{\varepsilon}-z_{0 \varepsilon}\right)^{n} .
\end{aligned}
$$

Para todo $n \in \mathbb{I N}$, consideremos $p_{n}:=-n-N-1$ e seja $k=2$. Então

$$
\lim _{n \rightarrow \infty}\left(p_{n}+k n\right)=+\infty \quad \text { e } \quad\left\|z-z_{0}\right\|<\frac{s}{2}<e^{-2}=e^{-|k|} .
$$

Seja $\eta_{1} \in \mathbf{I}$ tal que $\eta_{1}<\min \left\{r, \frac{1}{c}, \eta_{0}\right\}$. Então temos que

$$
\frac{1}{r^{n}}<\varepsilon^{-n} \quad \text { e } \quad c \varepsilon^{-N}=c \varepsilon \varepsilon^{-N-1}<c \frac{1}{c} \varepsilon^{-N-1}=\varepsilon^{-N-1}, \quad \forall \varepsilon \in \mathbf{I}_{\eta_{1}}, \forall n \in \mathbb{N},
$$

e, assim, por (4.16), temos que

$$
\left|a_{n \varepsilon}\right| \leq \frac{1}{r^{n}} c \varepsilon^{-N} \leq \varepsilon^{-n} \varepsilon^{-N-1}=\varepsilon^{-n-N-1}=\varepsilon^{p_{n}}, \quad \forall \varepsilon \in \mathbf{I}_{\eta_{1}}, \forall n \in \mathbb{N} .
$$

De (4.17), (4.18), (4.19) e da Proposição 1.4.8, concluímos que

$$
\kappa_{\Omega, \mathbb{C}}(f)(z)=\left[\left(\frac{1}{2 \pi i} \int_{\gamma_{\varepsilon}} \frac{f_{\varepsilon}(w)}{w-z_{\varepsilon}} d w\right)_{\varepsilon}\right]=\left[\left(\sum_{n=0}^{\infty} a_{n \varepsilon}\left(z_{\varepsilon}-z_{0 \varepsilon}\right)^{n}\right)_{\varepsilon}\right]=\sum_{n=0}^{\infty} a_{n}\left(z-z_{0}\right)^{n} .
$$

A prova do Teorema 4.2.10 aqui apresentada não é a encontrada em [AFJ-1], mas a extraída das Notas do Seminário Integrando funções generalizadas sobre Membranas, realizado no IME-USP (2005) e sob a responsabilidade de J. Aragona, R. Fernandez e S. O. Juriaans. 
Corolário 4.2.11 Sejam $\Omega$ um subconjunto aberto de $\mathbb{C}$ e $f \in \kappa_{\Omega, \mathbb{C}}(\mathcal{G}(\Omega ; \mathbb{C}))$. Então $f$ é analítica em $\widetilde{\Omega}_{c}$ se, e somente se, $f$ é holomorfa em $\tilde{\Omega}_{c}$.

Demonstração: É conseqüência do Teorema 3.2.11, da Proposição 4.2 .8 (3) e do Teorema 4.2.10.

A seguir, apresentamos uma caracterização para as funções de $\kappa_{\Omega, \mathbb{C}}(\mathcal{G}(\Omega ; \mathbb{C}))$ que são analíticas em $\widetilde{\Omega}_{c}$, onde $\Omega$ é um subconjunto aberto de $\mathbb{C}$.

Corolário 4.2.12 Seja $\Omega$ um subconjunto aberto de $\mathbb{C}$. Então

$$
\left\{f \in \kappa_{\Omega, \mathbb{C}}(\mathcal{G}(\Omega ; \mathbb{C})): f \text { é analítica em } \widetilde{\Omega}_{c}\right\}=\kappa_{\Omega, \mathbb{C}}(\mathscr{H} \mathcal{G}(\Omega)) \text {. }
$$

Demonstração: Segue do Corolário 4.2 .11 e da Proposição 4.2 .8 (3).

Finalizamos este trabalho mencionando que em [AFJ-1] encontram-se outros resultados que aqui não foram estudados, tais como um conceito de variedade generalizada e algumas aplicações, em equações diferenciais, dos resultados aqui abordados. 


\section{Índice de Símbolos e Notações}

$\mathbb{R}$

$\mathbb{R}_{+}$

$\mathbb{R}_{+}^{*}$

$\mathbb{C}$

$\mathbb{K}$

$\mathbb{I N}$

$\mathbb{N}^{*}$

$\mathbb{K}^{*}$

$A \backslash B$, onde $A \subset B$

Ker $T$

$\bar{X}$, onde $X \subset \mathbb{K}^{n}$ e $X \neq \mathbb{K}^{n}$

$B_{r}(x)$, onde $r \in \mathbb{R}_{+}^{*}$ e $x \in X$

$B_{r}^{\prime}(x)$, onde $r \in \mathbb{R}_{+}^{*}$ e $x \in X$

$S_{r}(x)$, onde $r \in \mathbb{R}_{+}^{*}$ e $x \in X$

$|a|$, onde $a \in \mathbb{K}^{n}$

$\beta \leq \alpha$, onde $\beta, \alpha \in \mathbb{N}^{n}$

$\left(\begin{array}{l}\alpha \\ \beta\end{array}\right)$, onde $\beta, \alpha \in \mathbb{N}^{n}$

$|\alpha|$, onde $\alpha=\left(\alpha_{1}, \ldots, \alpha_{1}\right) \in \mathbb{N}^{n} \quad \sum_{i=1}^{n} \alpha_{i}$ conjunto dos números reais

conjunto $\{x \in \mathbb{R}: x \geq 0\}$

conjunto $\{x \in \mathbb{R}: x>0\}$

conjunto dos números complexos

corpo $\mathbb{R}$ ou o corpo $\mathbb{C}$

conjunto dos números naturais

$\mathbb{I N} \backslash\{0\}$

$\mathbb{I K} \backslash\{0\}$

conjunto $\{a \in A: a \notin B\}$

núcleo do homomorfismo $T$

fecho de $X$ em $\mathbb{K}^{n}$

bola aberta, de centro $x$ e raio $r, \operatorname{em} X$

bola fechada, de centro $x$ e raio $r$, em $X$

esfera, de centro $x$ e raio $r$, em $X$

módulo de $a$ em $\mathbb{K}^{n}$

significa que $\beta_{i} \leq \alpha_{i}$, para todo $1 \leq i \leq n$, onde $\beta_{i}$ e $\alpha_{i}$ são a i-ésima coordenada de $\beta$ e $\alpha$, respectivamente $\prod_{i=1}^{n}\left(\begin{array}{c}\alpha_{i} \\ \beta_{i}\end{array}\right)$, onde $\beta_{i}$ e $\alpha_{i}$ são a i-ésima coordenada de $\beta$ e $\alpha$, respectivamente 


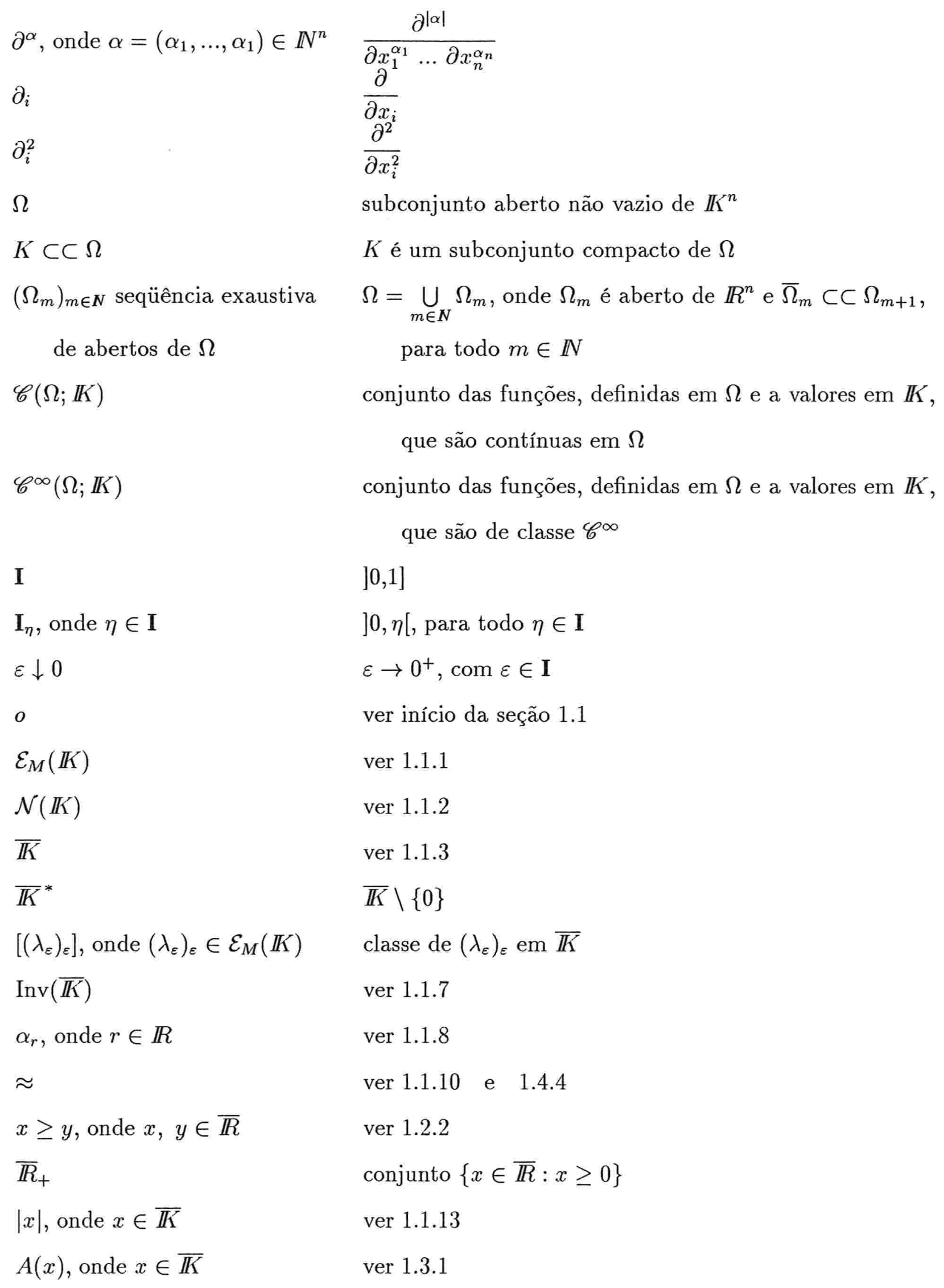




\begin{tabular}{|c|c|}
\hline $\mathcal{V}(x)$, onde $x \in \overline{\mathbb{I K}}$ & ver 1.3 .2 \\
\hline$\|\cdot\|$ & ver 1.3 .5 \\
\hline$D$ & ver 1.3 .5 \\
\hline $\mathscr{T}_{c}$ & ver 1.3 .9 \\
\hline$\|\cdot\|_{n}$ & ver 1.3 .10 \\
\hline$D_{n}$ & ver 1.3 .10 \\
\hline $\mathscr{T}_{c}^{n}$ & ver final da seção 1.3 \\
\hline$\sqrt{x}$, onde $x \in \overline{\mathbb{R}}_{+}$ & ver 1.4 .10 \\
\hline$[\cdot]_{2}$ & ver 1.4 .11 \\
\hline$\langle\cdot, \cdot\rangle$ & ver 1.4 .13 \\
\hline$\|F(\cdot)\|_{X}$ & ver início da seção 1.5 \\
\hline $\mathcal{E}[\Omega ; \mathbb{K}]$ & ver 1.5.1 \\
\hline $\mathcal{E}_{M}[\Omega ; \mathbb{K}]$ & ver 1.5 .2 \\
\hline $\mathcal{N}[\Omega ; \mathbb{K}]$ & ver 1.5 .3 \\
\hline $\mathcal{G}(\Omega ; \mathbb{K})$ & ver 1.5 .4 \\
\hline$\left[\left(f_{\varepsilon}\right)_{\varepsilon}\right]$, onde $\left(f_{\varepsilon}\right)_{\varepsilon} \in \mathcal{E}_{M}[\Omega ; \mathbb{K}]$ & classe de $\left(f_{\varepsilon}\right)_{\varepsilon}$ em $\mathcal{G}(\Omega ; \mathbb{I})$ \\
\hline$f_{x}$, onde $f \in \mathcal{G}(\Omega)$ e $x \in \Omega$ & ver 1.5 .13 \\
\hline$\Omega_{M}$ & ver 1.6.1 \\
\hline$\Omega_{c}$ & ver 1.6.1 \\
\hline$\sim$ & ver 1.6 .2 \\
\hline$\widetilde{\Omega}$ & ver 1.6 .3 \\
\hline$\widetilde{\Omega}_{c}$ & ver 1.6 .3 \\
\hline$f(x)$, onde $f \in \mathcal{G}(\Omega)$ e $x \in \widetilde{\Omega}_{c}$ & ver 1.6 .5 \\
\hline$|f|_{\beta, m}$ & ver 1.7 .2 \\
\hline$A_{m, p}(f)$, onde $f \in \mathcal{G}(\Omega)$ & ver 1.7.3 \\
\hline $\mathcal{V}_{m, p}(f)$, onde $f \in \mathcal{G}(\Omega)$ & ver 1.7.4 \\
\hline$D_{m, p}$ & ver 1.7 .7 \\
\hline $\mathscr{T}_{\Omega}$ & ver 1.7 .9 \\
\hline
\end{tabular}




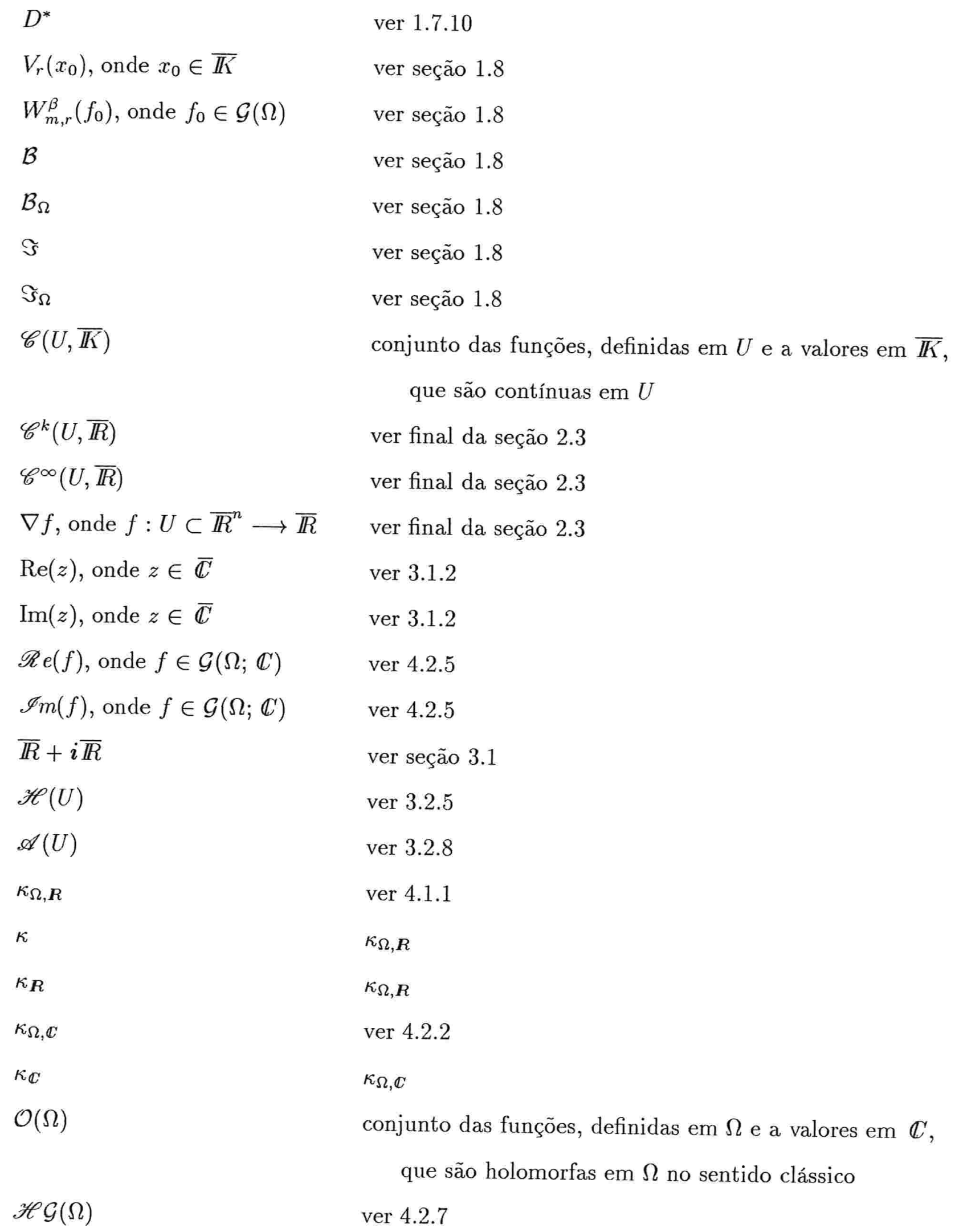




\section{Referências Bibliográficas}

[Ara-1] Aragona, J., Colombeau generalized functions on quasi-regular sets, Publ. Math. Debrecen, 68 (2006), 371-399.

[Ara-2] Aragona, J., Introdução à Teoria das Funções Generalizadas de Colombeau, Notas de aula da disciplina MAT829, Universidade de São Paulo, São Paulo, 1989.

[AB] Aragona, J.; Biagioni, H., Intrinsic definition of the Colombeau algebra of generalized functions, Anal. Math., 17 (1991), 75-132.

[AC] Aragona, J.; Colombeau, J. F., The interpolation theorem for holomorphic generalized functions, Ann. Polon. Math., 49 (1988), 151-156.

[AFJ-1] Aragona, J.; Fernandez R.; Juriaans, S. O., A discontinuous Colombeau differential calculus, Monatsh. Math., 144 (2005), 13-29.

[AFJ-2] Aragona, J.; Fernandez R.; Juriaans, S. O., Natural topologies on Colombeau Algebras, preprint.

[AFJ-3] Aragona, J.; Fernandez R.; Juriaans, S. O., The sharp topology on the full Colombeau algebra of generalized functions, Integral Transforms Spec. Funct., 17 (2006), 165-170.

[AJ] Aragona, J.; Juriaans, S. O., Some structural properties of the topological ring of Colombeau's generalized numbers, Comm. Algebra, 29 (2001), 2201-2230. 
[AJOS] Aragona, J.; Juriaans, S. O.; Oliveira O.; Scarpalezos D., Algebraic theory of the topological algebra of Colombeau's generalized functions, Proc. Edimburg Math. Soc. (to appear).

[AS] Aragona, J.; Soares, M., An existence theorem for an analytic first order PDE in the framework of Colombeau's theory, Monatsh. Math., 134 (2001), 9-17.

[AV] Aragona, J.; Villarreal, F., Colombeau's theory and shock waves in a problem of hydrodynamics, J. Anal. Math., 61 (1993), 113-144.

[Bia] Biagioni, H. A., A Nonlinear theory of generalized functions, Lecture Notes in mathematics, vol 1421, Springer-Verlag, 1990.

[Bou] Bourbaki, N., General Topology, Chapters 1-4 e Chapters 5-10, Springer-Verlag, Paris, 1989.

[Col] Colombeau, J. F., Elementary introduction to new generalized functions, NorthHolland, Amsterdam, 1985.

[CL] Colombeau, J.F.; Langlais, M., Generalized solutions of nonlinear parabolic equations with distributions as initial conditions, J. Math. Anal. Appl., 145 (1990), 186-196.

[CM] Colombeau, J.F.; Meril, A., Generalized functions and multiplication of distributions on $\mathscr{C}^{\infty}$ manifolds, J. Math. Anal. Appl., 186 (1994), 357-364.

[Con] Conway, J.B., Functions of One Complex Variable, Springer-Verlag, New York, 1975.

[Eng] ～Engelking, R., General Topology, Heldermann Verlag, Berlin, 1989.

[Fer] Fernandez R., A equação de Hamilton-Jacobi no contexto das funções generalizadas, Tese de Doutorado, Universidade de São Paulo, São Paulo, 1996. 
[Gar-G] Garcia, A. R. G., Os números e as funções generalizadas plena de Colombeau: aspectos algébricos, topológicos e analíticos, Tese de Doutorado, Universidade de São Paulo, São Paulo, 2006.

[Gar-C-1] Garetto, C., Microlocal analysis in the dual of a Colombeau algebra: generalized wave front sets and noncharacteristic regularity, New York J. Math., 12 (2006), $275-318$.

[Gar-C-2] Garetto, C., Pseudo-differential operators in algebras of generalized functions and global hypoellipticity, Acta Appl. Math., 80 (2004), 123-174.

[GKOS] Grosser, M.; Kunzinger, M.; Oberguggenberger, M.; Steinbauer, R., Geometric Theory of Generalized Functions with Applications to General Relativity, Kluwer Acadademic Publishers, vol 537, Netherlands, 2001.

[HO] Hörmann, G.; Oberguggenberger, M., Elliptic regularity and solvability for partial differential equations with Colombeau coefficients, Electron. J. Diff. Equations, 14 (2004), 1-30.

[Jur] Juriaans, S. O., Uma introdução à teoria das funções generalizadas de Colombeau, Minicurso, 64 Seminário Brasileiro de Análise, 2006.

[Kel] Kelley, J. L., Topología General, Editorial Universitaria de Buenos Aires, Buenos Aires, 1962.

[Kun] Kunzinger, M., Lie Transformation Groups in Colombeau algebras, Doctoral Thesis, University of Viena, 1996.

[KO] Kunzinger, M.; Oberguggenberger, M., Characterization of Colombeau generalized functions by their point values, Math. Nachr., 203 (1999), 147-157.

[KOSV] Kunzinger, M.; Oberguggenberger, M.; Steinbauer, R.; Vickers, J. A., Generalized flows and singular ODEs on differentiable manifolds, Acta Appl. Math., 80 (2004), 221-241. 
[KS] Kunzinger, M.; Steinbauer, R., Generalized pseudo-Riemannian geometry, Trans. Amer. Math. Soc., 354 (2002), 4179-4199.

[KSV] Kunzinger, M.; Steinbauer, R.; Vickers, J. A., Intrinsic characterization of manifold-valued generalized functions, Proc. London. Math. Soc., 87 (2003), 451-470.

[Lan] Lang, S., Algebra, Springer-Verlag, New York, 2002.

[Lim] Lima, E.L., Elementos de Topologia Geral, Livros Técnicos e Científicos, Rio de Janeiro, 1976.

[Mil] Milies, F.C.P., Anéis e Módulos, IME-USP, São Paulo, 1972.

[NPS] Nedeljkov, M.; Pilipović, S.; Scarpalezos, D., The linear Theory of Colombeau generalized functions, Pitman Research Notes in Mathematics Series, 385, Longman, 1998.

[NOP] Nedeljkov, M.; Oberguggenberger, M.; Pilipović, S., Generalized solutions to a semilinear wave equation, Nonlinear Anal., 61 (2005), 461-475.

[Obe] Oberguggenberger, M., Generalized functions and stochastics processes, Progress in Probability, 36 (1995), 215-229.

[OPV] Oberguggenberger, M.; Pilipović, S.; Valmorin, V., Global holomorphic representatives of Colombeau holomorphic generalized functions, preprint.

[Oli] Oliveira, E. R., Funções generalizadas em espaços de Banach. Um teorema do tipo Ovcyannikov-Treves e uma versão não linear do teorema de Ovcyannikov, Tese de Doutorado, Universidade de São Paulo, São Paulo, 2001.

[Pan] Panzarelli, H., Sobre a transformada de Hilbert no contexto das funçóes generalizadas, Tese de Doutorado, Universidade de São Paulo, São Paulo, 2004. 
[Ros] Rosinger, E. E., Non-linear partial differential equations. An algebraic view of generalized solutions, North Holland, Amsterdam, 1990.

[RR] Rosinger, E. E.; Rudolph M., Group invariance of global generalized solutions of smooth nonlinear PDEs: a Dedekind order completion method, Lie Groups Appl., 1 (1994), 203-215.

[RW] Rosinger, E. E.; Walus, Y. E., Group invariance of generalized solutions obtained through the algebraic method, Nonlinearity, 7 (1994), 837-859.

[Rud] Rudin, W., Princípios de Análise Matemática, Ao Livro Técnico S.A., Rio de Janeiro, 1971.

[Sca-1] Scarpalezos, D., Colombeau's generalized functions: topological structures microlocal properties. A simplified point of view, CNRS-URA212, Université Paris, $7,1993$.

[Sca-2] Scarpalezos, D., Topologies dans les espaces de nouvelles fonctions generalisées de Colombeau. $\overline{\mathbb{C}}$-modules topologiques, Université Paris, 7, 1993.

[Sil-A] Silva, A. A., Sobre a álgebra simplificada das funções generalizadas de Colombeau, Dissertação de Mestrado, Universidade de São Paulo, São Paulo, 2005.

[Sil-T] Silva, J. T., Sobre a resolubilidade local de operadores diferenciais parciais lineares no contexto das funções generalizadas, Tese de Doutorado, Universidade de São Paulo, São Paulo, 1995.

[Soa-G] Soares, M. G., Cálculo em uma variável complexa, IMPA, Rio de Janeiro, 2001.

[Soa-R] Soares, M. R., Sobre alguns problemas da teoria das funções holomorfas generalizadas, Tese de Doutorado, Universidade de São Paulo, São Paulo, 2000.

[SV] Steinbauer, R.; Vickers, J. A., The use of generalized functions and distributions in general relativity, Classical Quantum Gravity, 23 (2006), 91-144. 
[Vil-1] Villarreal, F., Composition for a class of generalized functions in Colombeau's theory, Integral Transforms Spec. Funct., 11 (2001), 93-100.

[Vil-2] Villarreal, F., Heaviside generalized functions and shock waves for a Burgers kind equation, Integral Transforms Spec. Funct., 17 (2006), 213-219.

[Vil-3] Villarreal, F., Sobre soluções na forma de onda de choque de certos sistemas de equações diferenciais parciais da hidrodinâmica, Tese de Doutorado, Universidade de São Paulo, São Paulo, 1990.

[Ver] Vernaeve, H., Embedding distributions in algebras of generalized functions with singularities, Monatsh. Math., 138 (2003), 307-318. 